\title{
CARS 2021: Computer Assisted Radiology and Surgery Proceedings of the 35th International Congress and Exhibition Munich, Germany, June 21-25, 2021
}

\section{Preface}

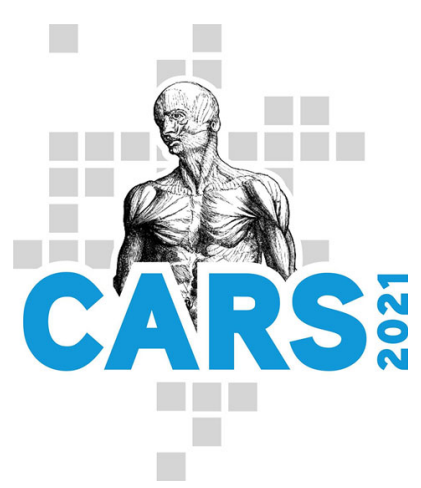

CARS 2021 and Rethinking the Hospital and OR of the Future

In addition to the traditional CARS topics and presentations, this year's CARS Congress is revisiting and widening its scope towards both, the Digital Operating Room of the Future and the Hospital of the Future. This is expressed in the CARS 2021 overall theme: "Rethinking the Hospital and OR".

In past CARS Congresses these themes have been addressed only as focused events, the "Hospital of the Future" as early as in 1995 [1] and the "OR 2020-The Operating Room of the Future" in CARS 2004 in close cooperation with Kevin Cleary and other colleagues in the USA [2], see Fig. 1. This resulted for example, also in the foundation of WG 24 DICOM in Surgery and the IHE Surgery domain.

CARS being mainly concerned about the presence and future of the clinical domains of radiology and surgery recognizes, however, that fundamental developments in IT impact not only these two specific disciplines, but also the hospital of the future and even the healthcare system as a whole, in which radiology and surgery are expected to play a major role.

Specifically, the goal of a newly established workshop for CARS 2021 "OR 2030 and Beyond" is to identify the clinical and technical requirements for the next generation operating room. OR 2030 is a sequel to OR 2020 [2] but also addressing themes of related developments. The next 10 years with the horizon of 2030 are selected as a target timeframe, but with a possible glance also at what might be expected beyond this period. Below are some of the proposed themes the OR 2030 initiative will focus on in corresponding four working groups:

- Group 1: Surgical robotics.

- Group 2: Intraoperative imaging.

- Group 3: Surgical informatics.

- Group 4: Machine intelligence.

\section{Surgical robotics}

Advanced robotic systems for surgery continue to develop and will no doubt play a larger role in the future operating room. Robotic devices may also be one part of the digital "surgical cockpit" of the future. These devices may also be integrated with advanced imaging modalities or even be controlled by imaging data. This group will discuss these and related topics.

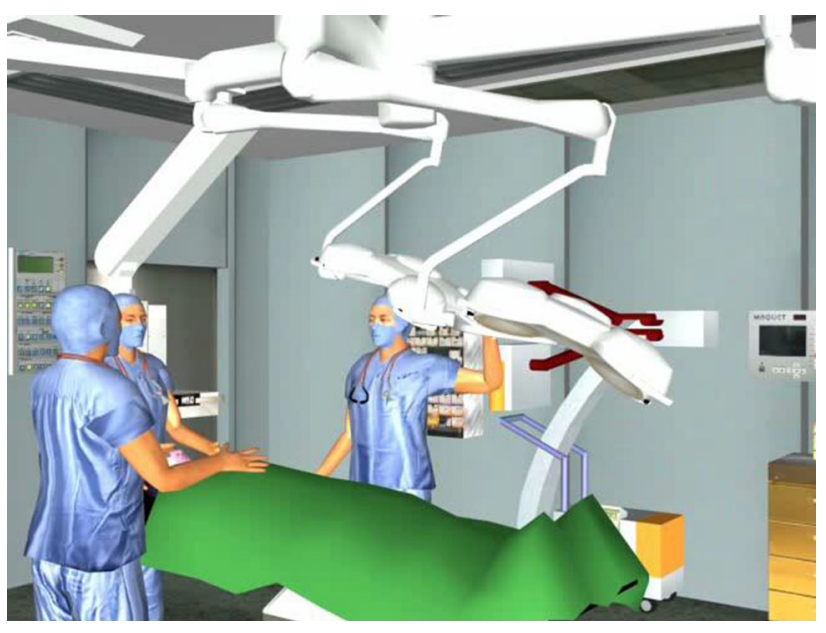

Fig. 1 Surgical workflow simulation in a virtual OR [2] 


\section{Intraoperative imaging}

Intraoperative imaging is becoming more and more widely used in the operating room, from the ubiquitous $\mathrm{C}$-arm to advanced systems such as cone-beam CT and intraoperative MRI. This group will discuss existing and new imaging modalities, including novel visualization methods and tools, and their role in the operating room. Image fusion and the use of multi-modality imaging will also be covered.

\section{Surgical informatics}

Surgical informatics is the collection, organization, and retrieval of biomedical information relevant to the care of the patient. This topic will not only include surgical data science but also surgical workflow, since a starting point for analyzing surgical procedures could be the workflow, and corresponding surgical simulation for education and training. Standards and common interfaces, including communications architectures for the OR, should also be discussed here.

\section{Machine intelligence and CARS}

As computational power, the availability of data sets, computational modeling methods and tools, and algorithmic advances continue to increase, machine intelligence is playing a large role in medicine. This working group will discuss how these advances will impact the future surgical workflow, surgical simulation, and the operating room. Synergistic interaction and collaboration between humans and intelligent machines is expected to be the major focus of this group.

In addition to the above workshop activities, some members of the CARS community have also been concerned about healthcare strategies for the employment of modern technologies, which aim at striking the right balance between quality, access for all and cost of healthcare [1], i.e. "The Iron Triangle of Health Care". Developments in technology relating to CARS greatly influence the result of these endeavours, but other factors coming from economics, hospital management and architecture, sociology, epidemiology, philosophy, etc. must also be considered.

If these observations deserve high importance ratings, it may be opportune to design and investigate possible future healthcare scenarios for different points in time. Specifically, to address those questions with respect to CARS that provoke thoughts on the future of healthcare for all stakeholders involved, i.e. the healthcare providers, healthcare industry and patients. Below are some examples of major themes and corresponding working groups which are planned to become part of CARS 2021 and beyond:

- Group 1: Smart Hospital.

- Group 2: Cross-disciplinary Care.

\section{Smart hospital}

With the implementation of intelligent sensors, trackers, natural language and gesture processing systems that are based on digital infrastructure information, a smart and assistive hospital becomes a realizable prospect. This group will not only discuss what type of data would be of particular interest and by which means information and knowledge (i.e. appropriate models) could be gathered, but also how intelligent systems based on these categories of understanding could

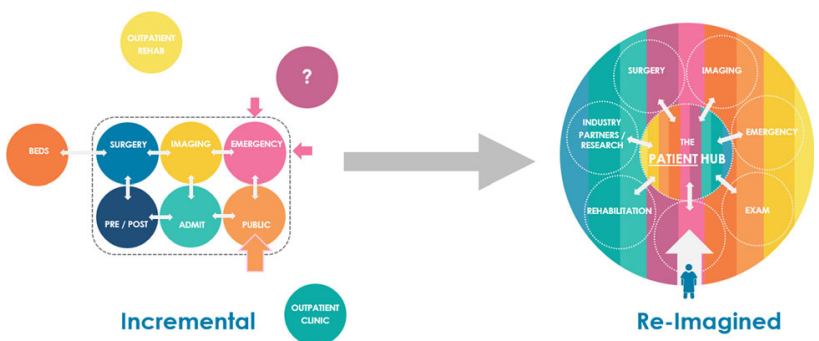

Fig. 2 Instead of treating patients in different departments separately, an integrated cross-disciplinary approach is anticipated for the hospital of the future [3]

improve patient care, or how they could impact the work profile of healthcare workers.

\section{Cross-disciplinary care}

The integration of different disciplines to improve patient care pays off not only in the operating room, e.g. during hybrid approaches, but is also proven efficient for other indications, for example for trauma management. Reuniting different medical fields and orchestrating them for the realization of patient centered approaches could revolutionize healthcare. This would require assistive methods and tools, a modification of the hospital design as well as platforms for crossdisciplinary collaboration, see Fig. 2. This group will discuss these and related topics.

Finally, we should like to thank the enablers of the hybrid CARS 2021 Congress, in particular our Munich colleagues Nassir Navab, Hubertus Feussner, Daniel Ostler, Alois Knoll and Tim Lüth and all their assistants, but also the authors who submitted a video version of their presentations. As we expect a stimulating discussion on the aforementioned topics during the virtual part of CARS 2021 Conference, we look forward continuing the discussion (in-presence) on the "Hospital and OR of the Future" in subsequent workshops (exploratory) later in the year 2021 and beyond.

Heinz U. Lemke, PhD and Dirk Wilhelm, MD

Munich, May 2021

\section{References}

[1] S. Dangelat, H. Lngruber, W. Kroy, W. D. Rauh, H. Rüden, H. U. Lemke (1995) Hospital of the Future- the Good, the Bad and the Ugly, Proceedings of CAR'95 Berlin, Springer.

[2] K. Cleary, S.K. Mun (2005) OR 2020 workshop report: Operating room of the future, Article in International Congress Series, May 2005, https://doi.org/10.1016/j.ics.2005.03.279. Published by Elsevier B.V.

(3) C. Amato, L. McCanne, R. Yang, D. Ostler, O. Ratib, D. Wilhelm, L. Bernhard (2021) The Hospital of the Future, Proceedings of CARS 2021 Munich, Springer. 
CARS 2021

Computer Assisted Radiology and Surgery

Proceedings of the 35th International Congress and Exhibition Munich, Germany, June 21-25, 2021 
CARS 2021

\section{Computer Assisted Radiology and Surgery}

\section{Proceedings of the 35th International Congress and Exhibition Munich, Germany, June 21-25, 2021}

Editors:

Professor Heinz U. Lemke, PhD

International Foundation for

Computer Assisted Radiology and Surgery

Klettgaustrasse 20

79790 Kuessaberg, Germany

Professor Leonard Berliner, MD

Staten Island University Hospital

Northwell Health

Department of Radiology

475 Seaview Avenue

Staten Island, NY 10305, USA

Professor Cristian A. Line, PhD

Rochester Institute of Technology

Biomedical Modeling, Visualization and

Image-guided Navigation Laboratory

160 Lomb Memorial Dr.

Rochester, NY 14623, USA

Managing Editor (Editorial Office):

Franziska Schweikert

International Foundation for

Computer Assisted Radiology and Surgery

Klettgaustrasse 20

79790 Kuessaberg, Germany
Professor Kensaku Mori, PhD

Nagoya University

Graduate School of Information Science

Information and Communications Headquarter

1 Furo-cho, Chikusa-ku

Nagoya 464-8601, Japan

Professor Dirk Wilhelm, MD

Klinikum rechts der Isar der TUM

Klinik und Poliklinik für Chirurgie

Ismaninger Str. 22

81675 Munich, Germany

Professor Hiroyuki Yoshida, PhD

Harvard Medical School

Massachusetts General Hospital

Department of Radiology

Suite 400C, 25 Chardon Street

Boston, MA 02114, U.S.A. 


\section{CARS 2021 President}

Dirk Wilhelm, MD

Klinikum rechts der Isar der TUM, Munich (DE)

\section{CARS 2021 General Chairs}

Hubertus Feussner, MD

Klinikum rechts der Isar der TUM, Munich (DE)

Alois Knoll, PhD

Technical University of Munich, Garching (DE)

Alexander Lenz, PhD

Technical University of Munich, Garching (DE)

Tim Lüth, PhD

Technical University of Munich, Garching (DE)

\section{CARS 2021 Scientific Medical Committee}

Rickmer F. Braren, MD

Klinikum rechts der Isar der TUM, Munich (DE))

Rüdiger von Eisenhart-Rothe, MD

Klinikum rechts der Isar der TUM, Munich (DE)

Marcus Makowski, MD

Klinikum rechts der Isar der TUM, Munich (DE))

\section{CARS Congress Organizing Committee}

Christos Angelopoulos, DDS

Columbia University, New York, NY (US)

Elizabeth Beckmann, BSc

Lanmark, Beaconsfield (GB)

Leonard Berliner, MD

Staten Island University Hospital, New York, NY (US)

Ulrich Bick, MD

Charité-University Medicine Berlin (DE)

Davide Caramella, MD

University of Pisa (IT)

Kevin Cleary, PhD

Children's National Medical Center, Washington, DC (US)

Mario A. Cypko, PhD

German Heart Institute Berlin (DE)

Takeyoshi Dohi, PhD

Tokyo Denki University (JP)

Kunio Doi, PhD

Chicago, IL (US)

Volkmar Falk, MD, PhD

German Heart Institute Berlin (DE)

Allan G. Farman, PhD, DSc

Chicago, IL (US)

Hubertus Feussner, MD

Klinikum rechts der Isar, Munich (DE)

Guy Frija, MD

Georges Pompidou European Hospital, Paris (FR)

Miguel Á. González Ballester, PhD

ICREA-Pompeu Fabra University, Barcelona (ES)

Makoto Hashizume, MD, PhD, FACS

Kitakyushu Koga Hospital, Fukuoka (JP)

Yoshihiko Hayakawa, PhD

Kitami Institute of Technology, Hokkaido (JP)

Javier Herrero Jover, MD, PhD

Centro Médico Teknon, Barcelona (ES)

David Hilderbrand

Lakeway, TX (US)
Petra Mela, PhD

Technical University of Munich, Garching (DE)

Nassir Navab, PhD

Technical University of Munich, Garching (DE)

Daniel Ostler, MSc

Klinikum rechts der Isar der TUM, Munich (DE)

Thomas Wendler, PhD

Technical University of Munich, Garching (DE)

Bernhard C. Meyer, MD

Klinikum rechts der Isar der TUM, Munich (DE)

Barbara Wollenberg, MD

Klinikum rechts der Isar der TUM, Munich (DE)

Pierre Jannin, PhD

University of Rennes (FR)

Leo Joskowicz, PhD

The Hebrew University of Jerusalem (IL)

Shoji Kido, MD, PhD

Osaka University Graduate School of Medicine (JP)

Heinz U. Lemke, PhD (Chair)

International Foundation for CARS, Kuessaberg (DE)

Kensaku Mori, PhD

Nagoya University (JP)

Yoshihiro Muragaki, MD, PhD

Tokyo Women's Medical University (JP)

Nassir Navab, PhD

Technical University Munich, Garching (DE)

Terry M. Peters, PhD

Robarts Research Institute, London, ON (CA)

Osman M. Ratib, MD, PhD, FAHA

University Hospital of Geneva $(\mathrm{CH})$

Hans G. Ringertz, MD, PhD

Karolinska Hospital, Stockholm (SE)

Yoshinobu Sato, PhD

Nara Institute of Science and Technology (JP)

Ramin Shahidi, PhD

Stanford University Medical Center (US)

Akinobu Shimizu, PhD

Tokyo University of Agriculture and Technology (JP)

Gabriele von Voigt, $\mathbf{P h D}$

Leibniz University Hannover (DE)

Dirk F. Wilhelm, MD

Klinikum rechts der Isar der TUM, Munich (DE)

Hiroyuki Yoshida, PhD

Harvard Medical School, Boston, MA (US) 
Past Presidents

\begin{tabular}{|c|c|}
\hline CAR '89 & $\begin{array}{l}\text { Heinz Oeser, MD } \\
\text { Berlin (DE) }\end{array}$ \\
\hline CAR ‘91 & $\begin{array}{l}\text { Auguste Wackenheim, MD } \\
\text { Strasbourg (FR) }\end{array}$ \\
\hline CAR '93 & $\begin{array}{l}\text { J. Oscar M.C. Craig, MD } \\
\text { London (GB) }\end{array}$ \\
\hline CAR '95 & $\begin{array}{l}\text { Alexander R. Margulis, MD } \\
\text { San Francisco (US) }\end{array}$ \\
\hline CAR ‘97 & $\begin{array}{l}\text { Takahiro Kozuka, MD } \\
\text { Osaka (JP) }\end{array}$ \\
\hline CAR '98 & $\begin{array}{l}\text { Herbert Kaufmann, MD } \\
\text { Berlin (DE) }\end{array}$ \\
\hline CARS ‘99 & $\begin{array}{l}\text { Raffaella de Dominicis, MD } \\
\text { Florence (IT) }\end{array}$ \\
\hline CARS 2000 & $\begin{array}{l}\text { Pierre Rabischong, MD, PhD } \\
\text { Montpellier (FR) }\end{array}$ \\
\hline CARS 2001 & $\begin{array}{l}\text { Toyomi Fujino, MD, PhD } \\
\text { Tokyo (JP) }\end{array}$ \\
\hline CARS 2002 & $\begin{array}{l}\text { Kiyonari Inamura, PhD } \\
\text { Hyogo (JP) }\end{array}$ \\
\hline CARS 2003 & $\begin{array}{l}\text { H.K. Huang, DSc, FRCR (Hon.) } \\
\text { Los Angeles, CA (US) }\end{array}$ \\
\hline CARS 2004 & $\begin{array}{l}\text { Karl-Heinz Höhne, PhD } \\
\text { Hamburg (DE) }\end{array}$ \\
\hline CARS 2005 & $\begin{array}{l}\text { Martin Lipton, MD, FACR, FAHA } \\
\text { Los Angeles, CA (US) }\end{array}$ \\
\hline CARS 2006 & $\begin{array}{l}\text { Hans G. Ringertz, MD, PhD } \\
\text { Stockholm (SE) }\end{array}$ \\
\hline
\end{tabular}

\begin{tabular}{|c|c|}
\hline CARS 2007 & $\begin{array}{l}\text { Madjid Samii, MD } \\
\text { Hannover (DE) }\end{array}$ \\
\hline CARS 2008 & $\begin{array}{l}\text { Javier Herrero Jover, MD, PhD } \\
\text { Barcelona (ES) }\end{array}$ \\
\hline CARS 2009 & $\begin{array}{l}\text { Bernard L. Crowe, BA, MPH } \\
\text { Canberra, NSW (AU) }\end{array}$ \\
\hline CARS 2010 & $\begin{array}{l}\text { Osman M. Ratib, MD, PhD } \\
\text { Geneva }(\mathrm{CH})\end{array}$ \\
\hline CARS 2011 & $\begin{array}{l}\text { Michael M. Vannier, MD } \\
\text { Chicago, IL (US) }\end{array}$ \\
\hline CARS 2012 & $\begin{array}{l}\text { Davide Caramella, MD } \\
\text { Pisa (IT) }\end{array}$ \\
\hline CARS 2013 & $\begin{array}{l}\text { Nassir Navab, PhD } \\
\text { Munich (DE) }\end{array}$ \\
\hline CARS 2014 & $\begin{array}{l}\text { Makoto Hashizume, MD, PhD, FACS } \\
\text { Fukuoka (JP) }\end{array}$ \\
\hline CARS 2015 & $\begin{array}{l}\text { Javier Herrero Jover, MD, PhD } \\
\text { Barcelona (ES) }\end{array}$ \\
\hline CARS 2016 & $\begin{array}{l}\text { Davide Caramella, MD } \\
\text { Pisa (IT) }\end{array}$ \\
\hline CARS 2017 & $\begin{array}{l}\text { Javier Herrero Jover, MD, PhD } \\
\text { Barcelona (ES) }\end{array}$ \\
\hline CARS 2018 & $\begin{array}{l}\text { Volkmar Falk, MD, PhD } \\
\text { Berlin (DE) }\end{array}$ \\
\hline CARS 2019 & $\begin{array}{l}\text { Pierre Jannin, PhD } \\
\text { Rennes (FR) }\end{array}$ \\
\hline CARS 2020 & $\begin{array}{l}\text { Hubertus Feussner, MD } \\
\text { Munich (DE) }\end{array}$ \\
\hline
\end{tabular}

Program Committee

Purang Abolmaesumi, PhD

University of British Columbia, Vancouver, BC (CA)

Samir Aboul-Hosn, MD, PhD

Hospital Plató, Barcelona (ES)

Reza Aghaeizadeh Zoroofi, PhD

University of Tehran (IR)

Asma'a Al-Ekrish, MD

King Saud University, Riyadh (SA)

Paul R. Algra, MD, PhD

Medical Center Alkmaar (NL)

Russell J. Andrews, MD

NASA Ames Research Center, Los Gatos, CA (US)

Christos Angelopoulos, DDS

Columbia University, New York, NY (US)

Yoshinori Arai, PhD, DDS

Nihon University, Atsugi (JP)

Jumpei Arata, PhD

Kyushu University, Fukuoka (JP)

Eiichiro Ariji, DDS, PhD

Aichi-Gakuin University School of Dentistry, Nagoya (JP)

Paulo M. Azevedo-Marques, PhD

University of Sao Paulo, Ribeirao Preto (BR)

Frits H. Barneveld Binkhuysen, MD, PhD

Meander Medical Center, Soest (NL)

John S.H. Baxter, PhD

University of Rennes (FR)

Erwin Bellon, PhD

University Hospitals Leuven (BE)

Dirk Beyersdorff, MD

University Hospital Hamburg-Eppendorf (DE)

Silvio Diego Bianchi, MD

University of Torino (IT)

Ulrich Bick, MD

Charité-University Medicine Berlin (DE)

Uldis Bite, MD

Mayo Clinic, Rochester, MN (US)

Mario Ceresa, PhD

Pompeu Fabra University, Barcelona (ES)

Kiyoyuki Chinzei, PhD

AIST, Tsukuba (JP)

Kevin Cleary, PhD

Children's National Medical Center, Washington, DC (US)

D. Louis Collins, $\mathbf{P h D}$

McGill University, Montreal, QC (CA)

Marcos Costa Kurzhals, PhD

International University of Catalunya, Barcelona (ES)

Mario A. Cypko, PhD

German Heart Institute Berlin (DE)

Andreas Dietz, MD, PhD

University Hospital Leipzig (DE)

Adrienn Dobai, MD

Semmelweis University, Budapest (HU)

Kunio Doi, PhD

Chicago, IL (US)

André J. Duerinckx, MD, PhD

Howard University College of Medicine, Washington, DC (US)

Georg Eggers, MD, DMD, PhD

Weinheim Head Center (DE)

Randy E. Ellis, PhD

Queen's University, Kingston, ON (CA) 
Masahiro Endo, MD, PhD

Shizuoka Cancer Center (JP)

Uwe Engelmann, PhD

CHILI GmbH, Dossenheim/Heidelberg (DE)

Giuseppe Esposito, MD

Georgetown University Hospital, Washington, DC (US)

Caroline Essert, PhD

University of Strasbourg (FR)

Allan G. Farman, PhD, DSc

Chicago, IL (US)

Mansoor Fatehi, MD

Federico II University, Tehran (IR)

Josep Fernández-Bayó, PhD

UDIAT Diagnostic Center, Sabadell (ES)

Gabor Fichtinger, PhD

Queen's University, Kingston, ON (CA)

Martin Fiebich, PhD

University of Applied Sciences, Giessen (DE)

Michael H. Friebe, PhD

Otto-von-Guericke-University, Magdeburg (DE)

Masakatsu G. Fujie, PhD

Waseda University, Tokyo (JP)

Hiroshi Fujita, PhD

Gifu University Graduate School of Medicine (JP)

Shigekazu Fukuda, PhD

National Institute of Radiological Sciences, Chiba (JP)

Pablo Garcia Tahoces, PhD

University of Santiago de Compostela (ES)

Bernard Gibaud, PhD

University of Rennes (FR)

Maryellen L. Giger, $\mathbf{P h D}$

The University of Chicago, IL (US)

Enrique J. Gomez-Aguilera, PhD

Polytechnic University of Madrid (ES)

Miguel Á. González Ballester, PhD

ICREA-Pompeu Fabra University, Barcelona (ES)

Lixu Gu, PhD

Shanghai Jiaotong University $(\mathrm{CN})$

Horst K. Hahn, PhD

Fraunhofer MEVIS, Bremen (DE)

Hideaki Haneishi, PhD

Chiba University (JP)

Stefan Haßfeld, MD, MDS

University Hospital Dortmund (DE)

Yoshihiko Hayakawa, PhD

Kitami Institute of Technology, Hokkaido (JP)

Vicente Hernandez-Soler, MD, DDS

Valencia University, Alicante (ES)

Kenneth R. Hoffmann, PhD

State University of New York at Buffalo, NY (US)

Hiroshi Honda, MD, PhD

Kyushu University, Fukuoka (JP)

Jaesung Hong, PhD

Daegu Gyeongbuk Inst. of Science and Technology (KR)

Hidetaka Hontani, PhD

Nagoya Institute of Technology (JP)

Yimin $\mathbf{H u}, \mathbf{P h D}$

Cancer Institute Hospital, Beijing (CN)

Juan A. Hueto Madrid, MD

Vall d'Hebron University Hospital, Barcelona (ES)

Koji Ikuta, PhD

The University of Tokyo (JP)

Hiroshi Iseki, MD, PhD

Waseda University, Tokyo (JP)

Masayori Ishikawa, PhD

Hokkaido University, Sapporo (JP)
Hiroyasu Iso, MD

Osaka University Graduate School of Medicine (JP)

Stephan Jacobs, MD

German Heart Institute Berlin (DE)

Yulei Jiang, PhD

The University of Chicago, IL (US)

Nobuyuki Kanematsu, PhD

National Institute of Radiological Sciences, Chiba (JP)

Akitoshi Katsumata, DDS, PhD

Asahi University, Gifu (JP)

Shigehiko Katsuragawa, PhD

Teikyo University, Omuta (JP)

Zafrulla Khan, DDS, MS

James Graham Brown Cancer Center, Louisville, KY (US)

Bob B. Kiaii, MD, FRCS

London Health Sciences Centre, ON (CA)

Shoji Kido, MD, PhD

Osaka University Graduate School of Medicine (JP)

Ron Kikinis, MD

Harvard Medical School, Boston, MA (US)

Young Soo Kim, MD, PhD

Hanyang University Medical Center, Seoul (KR)

Yasutomi Kinosada, MD, PhD

Gifu University Graduate School of Medicine (JP)

Masaki Kitajima, MD, PhD

International University of Health and Welfare, Tokyo (JP)

Etsuko Kobayashi, PhD

Tokyo Women's Medical University (JP)

Yo Kobayashi, PhD

Waseda University, Tokyo (JP)

Cornelia Kober, PhD

Hamburg University of Applied Sciences (DE)

Yoshie Kodera, PhD

Nagoya University Graduate School of Medicine (JP)

Masahiko Koizumi, MD

Osaka University Hospital (JP)

Christian Krettek, MD

Medical School Hannover (DE)

Tomohiro Kuroda, PhD

Kyoto University Hospital (JP)

Florent Lalys, PhD

Therenva SAS, Rennes (FR)

Heinz U. Lemke, PhD

International Foundation for CARS (DE)

Lilian L.Y. Leong Fung, MBBS

Hong Kong College of Radiologists (HK)

Qiang Li, PhD

Chinese Academy of Sciences, Shanghai $(\mathrm{CN})$

Hongen Liao, PhD

Tsinghua University School of Medicine, Beijing (CN)

Cristian A. Linte, PhD

Rochester Institute of Technology (US)

Brent J. Liu, PhD

University of Southern California, Los Angeles, CA (US)

Xiongbiao Luo, PhD

Xiamen University $(\mathrm{CN})$

Iván Maciá Oliver, PhD

VICOMTech, San Sebastián (ES)

Francisco Maculé Beneyto, MD

Hospital Clinic, Barcelona (ES)

Koutaro Maki, DDS, PhD

Showa University School of Dentistry, Tokyo (JP)

Rüdiger Marmulla, MD

University Hospital of Heidelberg (DE)

Ken Masamune, PhD

Tokyo Women's Medical University (JP) 
Yoshitaka Masutani, PhD

Hiroshima City University (JP)

Yasushi Matsumura, MD, PhD

Osaka University Hospital (JP)

Andreas Melzer, MD, DDS

Universities Dundee and St. Andrews (GB)

Mamoru Mitsuishi, PhD

The University of Tokyo (JP)

Carlos Monserrat-Aranda, PhD

Polytechnic University of Valencia (ES)

Xavier Morandi, MD, PhD

University of Rennes (FR)

Kensaku Mori, PhD

Nagoya University (JP)

Parvin Mousavi, PhD

Queen's University, Kingston, ON (CA)

Seong K. Mun, PhD

Arlington Innovation Center Health Research (US)

Yoshihiro Muragaki, MD, PhD

Tokyo Women's Medical University (JP)

Kikkeri S. Nagesh, MDS

R.V. Dental College, Bangalore (IN)

Janne J. Näppi, PhD

Harvard Medical School, Boston, MA (US)

Valery Naranjo Ornedo, PhD

Polytechnic University of Valencia (ES)

Kwan-Hoong Ng, PhD

University of Malaya, Kuala Lumpur (MY)

Christopher Nimsky, MD

University Hospital Giessen and Marburg (DE)

Robert M. Nishikawa, PhD

University of Pittsburgh (US)

Fridtjof Nüsslin, PhD

Technical University Munich (DE)

Steffen Oeltze-Jafra, PhD

Otto-von-Guericke-University, Magdeburg (DE)

Isao Ohnishi, MD, PhD

International University of Health and Welfare, Chiba (JP)

Yoshiharu Ohno, MD, PhD

Kobe University Graduate School of Medicine (JP)

Minoru Ono, MD, PhD

The University of Tokyo (JP)

Kaan Orhan, DDS, PhD

Ankara University Faculty of Dentistry (TR)

José B. Pagador Carrasco, PhD

Minimally Invasive Surgery Centre Jesus Usón (ES)

Javier Pascau, PhD

University Carlos III, Madrid (ES)

Ewa Pietka, PhD

Silesian University of Technology, Gliwice (PL)

Andreas Pommert, PhD

University Hospital Hamburg-Eppendorf (DE)

Henri Primo, MS

Primo Medical Imaging Informatics, Chicago, IL (US)

Jörg Raczkowsky, PhD

University of Karlsruhe (DE)

Klaus Radermacher, PhD

Helmholtz-Institute for Biomedical Engineering, Aachen (DE)

Stephen J. Riederer, PhD

Mayo Clinic, Rochester, MN (US)

Maria J. Rupérez-Moreno, PhD

Polytechnic University of Valencia (ES)

José M. Sabater Navarro, PhD

University Miguel Hernandez, Elche (ES)

Hidetoshi Saitoh, PhD

Tokyo Metropolitan University of Health Sciences (JP)
Luisa F. Sánchez Peralta, PhD

Minimally Invasive Surgery Centre Jesús Usón (ES)

Patricia Sánchez-González, PhD

Polytechnic University of Madrid (ES)

Warren S. Sandberg, MD, PhD

Vanderbilt University Medical Center, Nashville, TN (US)

Duygu Sarikaya, PhD

University of Rennes (FR)

Richard M. Satava, MD

University of Washington Medical Center, Seattle, WA (US)

Yoshinobu Sato, PhD

Nara Institute of Science and Technology (JP)

Kurt Alexander Schicho, MD, PhD

Vienna General Hospital (AT)

Wolfgang Schlegel, $\mathbf{P h D}$

German Cancer Research Center, Heidelberg (DE)

Robert A. Schmidt, MD

University of Chicago, IL (US)

Armin Schneider, PhD

Klinikum rechts der Isar, Munich (DE)

Alexander Schramm, MD, DDS, PhD

Bundeswehrkrankenhaus Ulm (DE)

Rainer Schubert, MD

University for Health Sciences, Hall (AT)

Dirk Schulze, MD, DMD

Digitales Diagnostikzentrum Breisgau, Freiburg (DE)

Achim Schweikard, PhD

University of Lubeck (DE)

Ramin Shahidi, PhD

Stanford University Medical Center (US)

Mitsuo Shimada, MD, PhD

Tokushima University (JP)

Akinobu Shimizu, PhD

Tokyo University of Agriculture and Technology (JP)

Amber Simpson, PhD

Queen's University, Kingston, ON (CA)

Eduardo Soudah, PhD

Polytechnic University of Catalonia, Barcelona (ES)

Stefanie Speidel, $\mathbf{P h D}$

National Center for Tumor Diseases, Dresden (DE)

Matthäus Stöhr, MD

University Hospital Leipzig (DE)

Geert J. Streekstra, PhD

Academic Medical Center, Amsterdam (NL)

Nobuhiko Sugano, MD, PhD

Osaka University Graduate School of Medicine (JP)

Kazuro Sugimura, MD

Kobe University Graduate School of Medicine (JP)

Tae Suk Suh, PhD

The Catholic University of Korea, Seoul (KR)

Iori Sumida, PhD

Osaka University Graduate School of Medicine (JP)

Russell H. Taylor, PhD

Johns Hopkins University, Baltimore, MD (US)

Ulf K.-M. Teichgräber, MD, PhD

University Hospital Jena (DE)

Bart M. ter Haar Romeny, PhD

Eindhoven University of Technology (NL)

Klaus D. Toennies, PhD

Otto-von-Guericke-University, Magdeburg (DE)

Thomas Tolxdorff, PhD

Charité-University Medicine Berlin (DE)

Noriyuki Tomiyama, MD, PhD

Osaka University Graduate School of Medicine (JP)

Fukai Toyofuku, PhD

Kyushu University, Fukuoka (JP) 
Jocelyne Troccaz, PhD

University of Grenoble, La Tronche (FR)

Jayaram K. Udupa, PhD

University of Pennsylvania, Philadelphia, PA (US)

Tamas Ungi, PhD

Queen's University, Kingston, ON (CA)

Michiel W.M. van den Brekel, MD, PhD

The Netherlands Cancer Institute, Amsterdam (NL)

Eric vanSonnenberg, MD

St. Joseph's Hospital and Medical Cent., Phoenix, AZ (US)

Gabriele von Voigt, PhD

Leibniz University Hannover (DE)

Kirby G. Vosburgh, PhD

CIMIT, Boston, MA (US)

Shinichi Wada, PhD

Niigata University Faculty of Medicine (JP)

Andreas Wahle, PhD

The University of Iowa (US)
Stefan Weber, PhD

University of Berne $(\mathrm{CH})$

Michael Wich, MD

Unfallkrankenhaus Berlin (DE)

Dirk Wilhelm, MD

Klinikum rechts der Isar der TUM, Munich (DE)

Takaharu Yamazaki, PhD

Saitama Institute of Technology (JP)

Guang-Zhong Yang, PhD

Shanghai Jiao Tong University $(\mathrm{CN})$

Byung-Ju Yi, PhD

Hanyang University, Ansan (KR)

Hiroyuki Yoshida, PhD

Harvard Medical School, Boston, MA (US)

Toshiki Yoshimine, MD

Osaka University Graduate School of Medicine (JP) 


\section{Computer Assisted Radiology and Surgery- 35th International Congress and Exhibition}

Chairs: Ulrich Bick, $M D(D E)$, Dirk Wilhelm, $M D(D E)$ 


\section{Image reconstruction using end-to-end deep learning for low-dose CT}

D. Bauer ${ }^{1}$, C. Ulrich ${ }^{1}$, A. K. Golla ${ }^{1}$, T. Russ ${ }^{1}$, C. Tönnes ${ }^{1}$, J. Leuschner $^{2}$, M. Schmidt ${ }^{2}$, L. Schad ${ }^{1}$, F. Zöllner ${ }^{1}$

${ }^{1}$ Heidelberg University, Chair in Computer Assisted Clinical Medicine, Mannheim, Germany ${ }^{2}$ University of Bremen, Center for Industrial Mathematics, Bremen, Germany

Keywords Image Reconstruction, Computed Tomography (CT), Low-dose, Deep Learning

\section{Purpose}

The development of techniques for dose reduction is a major research topic in CT imaging, as it reduces the potential risk of cancer induction from CT radiation. To reduce the CT dose it is necessary to lower the overall photon count. However, this leads to a deterioration of image quality, as the acquired projection data is noisy. Classical iterative reconstruction algorithms are able to provide images of increased quality compared to a conventional filtered back projection (FBP). However, they do not perform robustly with heavily undersampled data and the computation is time-intensive due to repeated forward and back projection operations.

We present a fast end-to-end deep learning reconstruction method trained on the Low-Dose Parallel Beam (LoDoPaB)-CT challenge dataset [1]. The network performs the entire image reconstruction from the sinogram to the image. The network imitates all typical steps of a conventional image reconstruction. The sinogram is refined and filtered before being back projected into the image space, where the images are further refined. We compare the reconstructions of our network to the FBP and to a learned iterative method called Learned Primal-Dual, which won the LoDoPaB-CT challenge.

\section{Methods}

The concept of our network is based on the iCT-Net by Li et al. [2], which in turn is inspired by the common filtered back projection. The reconstruction process is learned end-to-end, i.e. the network input is the sinogram and the output is the reconstructed image. The full network architecture is displayed in Fig. 1.

First, disturbances in the raw measurement data, such as excessive noise or beam hardening, are suppressed as much as possible via $3 \times 3$ convolutions (refining layers). Using $10 \times 1$ convolutions, the corrected sinogram is then filtered (filtering layers). The filtered sinogram has the same size as the input sinogram. Afterwards, a back projection step projects the refined and filtered sinogram data into the image space. This is realized by a $\mathrm{d} \times 1$ convolution with $\mathrm{N}^{2}$ output channels without padding, where $\mathrm{d}$ is the number of detectors in the sinogram and $\mathrm{N}$ is the output image size. This convolution corresponds to a fully connected layer for each viewing angle, as it connects every detector element with every pixel in the image space. Thus, this network can be trained with sinograms acquired with different beam geometries, such as fan beam and parallel beam. Afterwards, the results for each view are reshaped to NxN sized

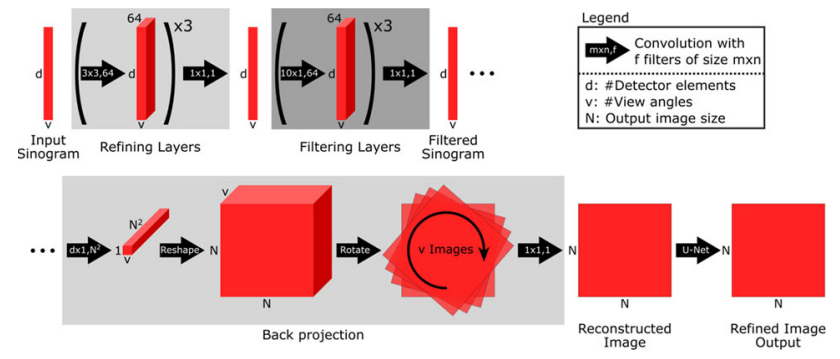

Fig. 1 End-to-end CT reconstruction network architecture images and rotated according to the acquisition angle. The rotated images are linearly interpolated and cropped to maintain an image size of $\mathrm{NxN}$. The back projected image is then obtained by combining all views with a $1 \times 1$ convolution. Finally, a U-Net is used for further refinement of the image output.

As training data for our network we used the 35,820 sample pairs of the publicly available LoDoPaB dataset. A sample pair consists of a $362 \times 362$ pixel CT image and a low-dose sinogram with 513 projection beams in parallel-beam geometry and 1000 projection angles. Simulated Poisson-distributed noise was added to the sinogram based on a photon count of 4096 per detector pixel before attenuation. To considerably lower the GPU memory requirements, we downsampled the sinograms to 500 projection angles. Training was performed with a SSIM loss function. The reconstructions were evaluated using the peak signal-to-noise ratio (PSNR) and structural similarity (SSIM) on 3678 challenge sample pairs.

\section{Results}

In Fig. 2 a reconstructed slices using the filtered back projection and our network are compared to the ground truth. The FBP is very noisy due to the noise in the sinogram data. In contrast, the reconstruction of our network is virtually noise free, as can be seen by the homogeneous liver. Slight over-smoothing is observed, which might lead to blurring of objects. To avoid this, we will consider to reduce the impact of the U-Net in the future. The SSIM and PSNR evaluation metrics for the FBP, our network and Learned Primal-Dual are shown in Table 1. Much higher SSIM and PSNR demonstrate that our network provides superior reconstructions compared to the FBP. While our network is only slightly worse than Learned Primal-Dual in terms of SSIM, the PSNR is substantially lower. The quality of our reconstruction could be increased if we used the full 1000 views instead of only 500. Furthermore, for training we only used the simulated noisy sinograms of the LoDoPaB dataset. To enhance the performance of our network, the (non-public) noiseless sinograms could be used to pre-train the refining and filtering layers. Although our approach slightly underperformed compared to a state-of-the-art method for high noise scenarios, we expect our network to provide competitive results even with very sparse projection data. We will investigate this in a subsequent study.
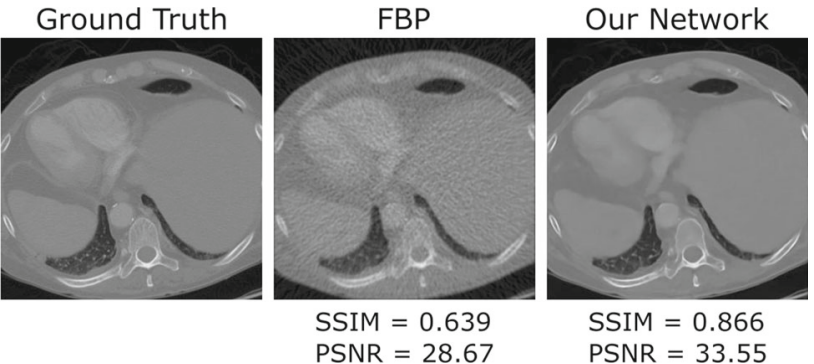

Fig. 2 Reconstructions using the filtered back projection and our network compared to the ground truth

Table 1 SSIM and PSNR evaluation metrics for different reconstruction techniques

\begin{tabular}{lll}
\hline Method & PSNR $\uparrow$ & SSIM $\uparrow$ \\
\hline FBP & $30.2 \pm 2.6$ & $0.73 \pm 0.13$ \\
Our network & $33.7 \pm 2.8$ & $0.85 \pm 0.12$ \\
Learned primal-dual & $36.3 \pm 3.7$ & $0.87 \pm 0.12$ \\
\hline
\end{tabular}




\section{Conclusion}

We presented an end-to-end deep learning CT reconstruction algorithm, which was superior to a common FBP for low-dose image reconstruction. In the future the ability of the network to mitigate metal artefacts will be investigated, by training it with sinograms containing simulated metal signals.

\section{References}

[1] Leuschner J, Schmidt M, Baguer DO, Maaß P (2019) The lodopab-ct dataset: A benchmark dataset for low-dose CT reconstruction methods. arXiv preprint arXiv:1910.01113.

[2] Li Y, Li K, Zhang C, Montoya J, Chen GH (2019) Learning to reconstruct computed tomography images directly from sinogram data under a variety of data acquisition conditions. IEEE transactions on medical imaging, 38(10), 2469-2481.

\section{Micro-CT-assisted cross-modality super-resolution of clinical CT: utilization of synthesized training dataset}

T. Zheng ${ }^{1}$, H. Oda ${ }^{2}$, S. Nakamura ${ }^{2}$, M. Mori ${ }^{3}$, H. Takabatake ${ }^{4}$, H.

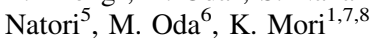

${ }^{1}$ Nagoya University, Graduate School of Informatics, Nagoya, Japan

${ }^{2}$ Nagoya University, Graduate School of Medicine, Nagoya, Japan

${ }^{3}$ Sapporo Kosei General Hospital, Sapporo, Japan ${ }^{4}$ Sapporo Minamisanjo Hospital, Sapporo, Japan ${ }^{5}$ Keiwakai Nishioka Hospital,

Sapporo, Japan ${ }^{6}$ Nagoya University, Information and Communications, Nagoya, Japan ${ }^{7}$ Nagoya University, Information Technology Center, Nagoya, Japan ${ }^{8}$ National Institute of Informatics, Research Center of Medical BigData, Tokyo, Japan

Keywords Unpaired super-resolution, Microstructure reconstruction, Multi-modality image synthesis, Lung cancer CT

\section{Purpose}

This paper proposes an unsupervised super-resolution (SR) method, which increases clinical CT resolution into the micro-focus X-ray CT $(\mu \mathrm{CT})$-level. Due to the resolution limit of clinical CT, it is challenging to observe pathological information at the alveoli level. $\mu \mathrm{CT}$ scanning allows the acquisition of lung with much higher resolution. Thus, SR of clinical CT volumes to $\mu$ CT-level is expected to help diagnosis of lung disease.

Typical SR methods require pairs of low-resolution (LR) and highresolution (HR) dataset for training. Unfortunately, aligning paired clinical CT (LR) and $\mu \mathrm{CT}$ (HR) volumes of human tissues is infeasible. Thus, we resuire an unsupervised SR method, which utilizes unpaired dataset of LR an HR images. Previous unsupervised method named SR-CycleGAN [1] is be hard to convergence in the training phase, because SR-CycleGAN needs to perform SR and domain translation in a single network. We tackle this problem by proposing a two-stage approach. First stage is building corresponding $\mu \mathrm{CT}$ and clinical CT pairs dataset by synthesizing clinical CT volumes from $\mu \mathrm{CT}$ volumes. Second stage, we use simulated clinical CT $-\mu \mathrm{CT}$ volume pairs from the dataset to train an FCN (fully convolutional network) for SR of clinical CT volumes. Proposed method outperformed SR-CycleGAN both quantitatively and qualitatively.

The contributions of our proposed method are: (1) trans-modality super-resolution from clinical CT to $\mu \mathrm{CT}$-level utilizing synthesizing training dataset and (2) an SR approach for clinical CT to $\mu$ CT-level that works without any paired clinical $\mathrm{CT}-\mu \mathrm{CT}$ data.

\section{Methods}

\section{Overview}

Given a set of clinical CT volumes and $\mu \mathrm{CT}$ volumes, the method would learn to perform SR of a clinical CT volume $x$ into a $\mu \mathrm{CT}$ like SR volume $\boldsymbol{x}^{\mathrm{SR}}$. The method consists of two stages. The first stage is building synthesized clinical CT- $\mathrm{CCT}$ dataset $\boldsymbol{D}$ using a synthesizing network. The second stage is a SR network trained on the dataset $\boldsymbol{D}$, which learns a mapping from clinical volumes $\boldsymbol{x}$ (LR volumes) to $\mu \mathrm{CT}$ volumes $\boldsymbol{y}$ (HR volumes).

For inference, a clinical CT volume $\boldsymbol{x}$ is input into the SR network and we will obtain volume $\boldsymbol{x}^{\mathrm{SR}}$ as the SR output.

\section{Stage 1: Building synthesized clinical CT- $-\mu \mathrm{CT}$ dataset}

We design a synthesizing network which generates synthesize clinical CT-like volumes from $\mu \mathrm{CT}$ volumes. The synthesizing network is designed following CycleGAN [2] with additional loss terms based on SSIM. We train the synthesizing network using clinical CT and downsampled $\mu \mathrm{CT}$ volumes. We use trained synthesizing network for synthesizing clinical CT-like volumes $\boldsymbol{x}$ ' from $\mu \mathrm{CT}$ volumes $\boldsymbol{y}$. Large amount of paired $\boldsymbol{x}^{\prime}$ and $\boldsymbol{y}$ forms synthesized clinical CT- $-\mu \mathrm{CT}$ dataset $\boldsymbol{D}$.

Stage 2: Training SR network using synthesized clinical CT$\mu \mathrm{CT}$ dataset

By building synthesized clinical CT $-\mu \mathrm{CT}$ dataset $\boldsymbol{D}$, we are able to train a SR network $f$ using clinical CT-like volumes $\boldsymbol{x}$ ' as input and $\mu \mathrm{CT}$ volumes $\boldsymbol{y}$ as corresponding ground truth. We use an FCN as the network for SR. We use pixel-wise $l_{2}$ loss as loss function between network output $f\left(\boldsymbol{x}^{\prime}\right)$ and ground truth $\boldsymbol{y}$ to train the network.

To perform SR of a clinical CT volume, the clinical CT volume is splitted into patches of $n \times n$ (pixels) as input into trained SR network. Then we obtain SR output of $8 n \times 8 n$ (pixels). We combine multiple SR outputs as the final SR result of the whole CT volume. Results

In stage 1 , we used eight clinical $\mathrm{CT}$ volumes and six $\mu \mathrm{CT}$ volumes. In stage 2 , we used nine $\mu \mathrm{CT}$ volumes. For training each network in stage 1 and stage 2, 2000 2D patches were extracted from each clinical CT and $\mu \mathrm{CT}$ volume. The clinical CT volumes were acquired from lung cancer patients, with resolution $0.625 \times$ $0.625 \times 0.6 \mathrm{~mm}^{3} /$ voxel. The $\mu \mathrm{CT}$ volumes were of lung specimens resected from lung cancer patients, with resolutions in the range of $(42-52) \times(42-52) \times(42-52) \quad \mu^{3} /$ voxel. We implemented our proposed method with PyTorch.

The size of patches extracted from the clinical CT volumes were set as $32 \times 32$ pixels, while patches extracted from the $\mu \mathrm{CT}$ volumes were set as $256 \times 256$ pixels. SR was conducted eight-times enlargement for each axis $(32 \times 32$ to $256 \times 256$ pixels $)$.

We utilized two clinical CT volumes for qualitative evaluation. We compare the SR results with the previous method SR-CycleGAN [1]. We used four $\mu \mathrm{CT}$ volumes for quantitative evaluation, as shown in Table 1. The qualitative results are shown in Fig. 1. Compared with the SR-CycleGAN, the edge of tube structures are clearly shown in the SR results of the proposed method.

Table 1 Quantitative evaluation of previous method [1] and proposed method

\begin{tabular}{lcc}
\hline & Previous method [1] & Proposed method \\
\hline PSNR & 11.79 & $\mathbf{1 3 . 2}$ \\
SSIM & 0.36 & $\mathbf{0 . 6 8}$ \\
\hline
\end{tabular}



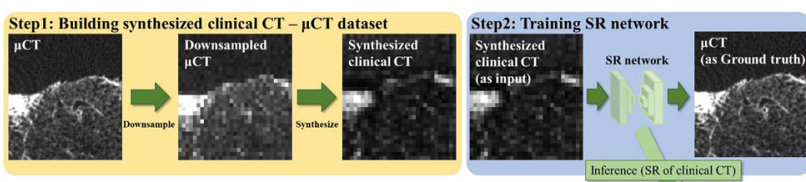

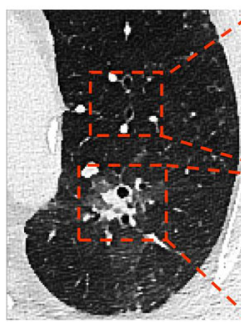

Right lung

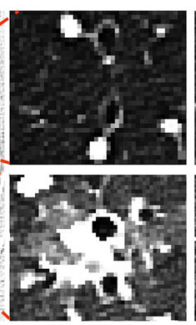

Clinical CT

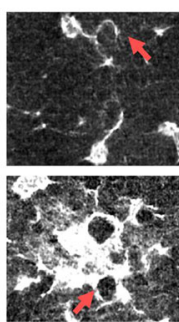

SR result (Previous method)

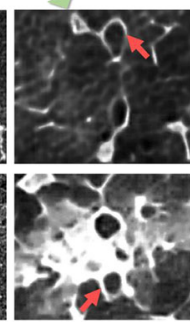

SR result (Proposed method)
Fig. 1 SR results of proposed method and previous method (SRCycleGAN). Upper half: generating synthesized clinical CT from $\mu \mathrm{CT}$ and training the SR network. Lower half: SR results. Compared with SR-CycleGAN, the edges of tube structures such as bronchus are clearly shown in the results of the proposed method (red arrows)

\section{Conclusion}

We proposed a SR method from clinical CT volumes to $\mu \mathrm{CT}$ level with synthesis of the training dataset. To the best of our knowledge, our proposed method is the first study that performs unsupervised SR with synthesized dataset.

Future work includes introduction of a more precise quantitative evaluation approach. Quantitative evaluation approach was conducted by evaluating how well synthesized clinical CT can be reconstructed to $\mu \mathrm{CT}$. We plan to make clinical $\mathrm{CT}-\mu \mathrm{CT}$ volume pairs by taking clinical CT volumes of lung specimen.

\section{References}

[1] Zheng $T$, Oda $H$, Moriya $T$, Sugino $T$, Nakamura S, Oda M, Mori M, Takabatake H, Natori H, Mori K (2020) Multi-modality super-resolution loss for GAN- based super-resolution of clinical CT images using micro CT image database. Prcoc. SPIE, Medical Imaging: 11313-3

[2] Zhu J.Y, Park T, Isola P, Efros A.A (2017) Unpaired image-toimage translation using cycle-consistent adversarial networks. Proceedings of the IEEE International Conference on Computer Vision: 2223-2232

\section{IRDBB: Archiving and communication model of curated imaging data for research and machine learning}

J. Spaltenstein ${ }^{1}$, G. Pasquier ${ }^{2}$, N. van Dooren ${ }^{1}$, P. Mildenberger ${ }^{3}$, B. Gibaud $^{2}$, O. Ratib ${ }^{1}$

${ }^{1}$ University of Geneva, Radiology and medical informatics, Genève, Switzerland ${ }^{2}$ University of Rennes, Faculté de Médecine, Rennes, France ${ }^{3}$ Johannes Gutenberg-Universität, Klinik fur Radiologie, Mainz, Germany

Keywords DICOM, Imaging Database, Imaging biobanks, Big Data

\section{Purpose}

With the development of a growing number of Artificial Intelligence (AI) and autonomous image analysis tools, there is a growing need for IT platforms that provide a structured approach to data management

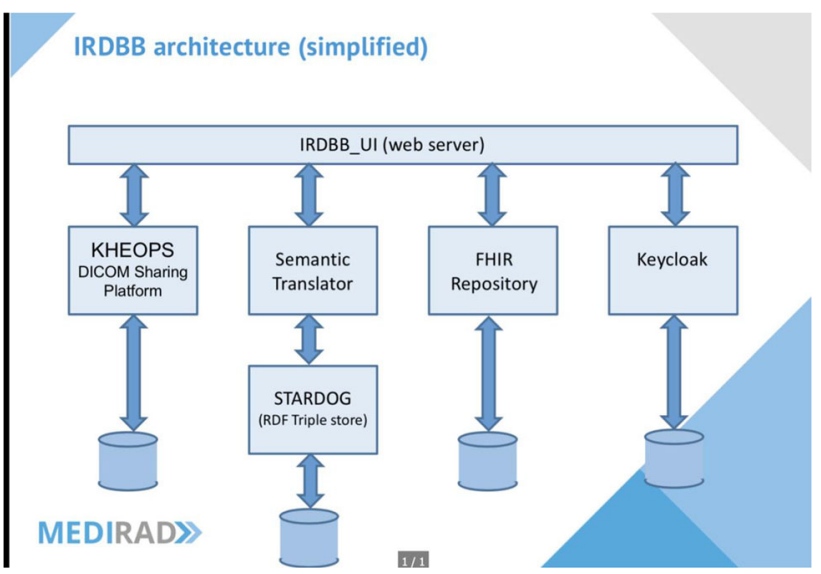

Fig. 1 General architecture of IRDBB architecture

and communication of curated imaging data that can serve for development and testing of a new generation of Deep Learning AI tools. Our goal was to develop an image archiving platform for DICOM and non-DICOM data that can be well referenced and retrieved using a classifier tool based on an ontology that can be adapted and designed to explicitly define all the information that is used for different analysis and research protocols.

\section{Methods}

A tailored implementation of such a model was developed for a platform dedicated to sharing imaging data, along with the associated dosimetric data pertaining to the radiation doses absorbed by specific organs. This platform called Imaging and Radiation Dose Biobank (IRDBB) was designed to support a multicentric European project (MEDIRAD ref.) for the development and validation of simulation models to measure the effects of low radiation dose in the context of medical procedures, see Fig. 1. An interactive platform allows users to assign specific ontology References to each dataset by which the data can be retrieved in selected cohorts based on a well-defined syntax.

\section{Results}

A first instance of such a model was implemented to host imaging data and analysis results in a common sharable database.

This IRDBB platform consists of the following components:

- A web server (IRDBB_UI) which manages user features for uploading of images and database requests;

- The open-source KHEOPS image archive platform that also provides DICOMweb access to the images for third-party analysis software and results reporting systems.

- A FHIR repository used to manage non-DICOM data;

- A semantic data manager, referred to as the Semantic Translator, used to translate the metadata of the imported images into a semantic graph whose elements (i.e. nodes and arcs) are classified according to the OntoMEDIRAD ontology designed specifically to standardize the semantics of information used by the MEDIRAD project;

- A semantic database implemented using the Stardog3 RDF triplestore;

- An Identity and Access Manager (IAM) implemented using Redhat"s Keycloak software.

Data generated by different groups of the project as well as results derived from specific analysis and modeling tools are hosted on the IRDBB server and can be retrieved through specific query templates based on the defined ontology.

A special extension to the platform called the "Report Provider" allows selected sets of data to be used by a remote server that 
performs specific analysis or processing functions that may return results in different format (i.e. graphs, tables, derived images or DICOM structured reports). This mechanism was specifically designed to allow data to be sent and processed by remote AI or image processing platforms.

\section{Conclusion}

The current implementation of our model of curated imaging data for research and machine learning has shown its ability to serve for multidisciplinary research and for the development of analysis tools on a variety of imaging data. It demonstrates the need for classification and curation of imaging data based on a standard ontology that defines the semantics used to query and retrieve data for specific research purposes. The web-based KHEOPS archive provides convenient access to the imaging data as well as web-services destined to analysis and AI tools residing on remote platforms.

This work serves as a proof of concept of a new architecture of large imaging databases (Big Data) that are becoming essential for future developments of $\mathrm{AI}$ and machine learning systems.

\section{Investigation of visibility of head-mounted display for vascular observation}

T. Shinohara ${ }^{1}$, A. Morioka ${ }^{1}$, N. Nakasako ${ }^{1}$

${ }^{1}$ Kindai University, Kinokawa-shi, Japan

Keywords visibility, head-mounted display, display resolution, vascular observation

\section{Purpose}

Three-dimensional (3D) representations of objects help us intuitively understand their shapes. The 3D representation of medical images obtained using several modalities, including computed tomography (CT) and magnetic resonance imaging, is indispensable for morphological and anatomical diagnoses and surgical treatments. However, organs with complicated structures, such as vasculatures, remain difficult to observe even if they are three-dimensionally displayed on an ordinal two-dimensional (2D) monitor. This is because their depth of the 3D representations is hardly recognized on the $2 \mathrm{D}$ monitor. A head-mounted display (HMD) is often used for medical purposes, such as image guidance and augmented reality in surgery. One of the advantages of using the HMD is to represent objects stereoscopically such that their depth can be easily recognized. In our previous study, we proposed a vascular virtual handling system [1] using which physicians can observe the vasculature stereoscopically using the HMD as the blood vessels appear more realistically, and handle blood vessels using a 3D pointing device for endovascular intervention assistance. An experiment to find a pseudo lesion embedded into the cerebral artery showed that the visibility of this system for vascular observation was superior to that of the system using an ordinal 2D monitor instead of the HMD [2]. This result indicates that the stereoscopic representation effectively represents objects with complicated structures such as vasculatures. This paper investigates the visibility of the HMD for vascular observation in more detail through an experiment similar to that in our previous study. Particularly, the relevance of the lesion size to the visibility of the HMD was investigated using pseudo lesions of three different sizes.

\section{Methods}

The HMD used in this study has a sensor to track head movement so that the user's viewpoint can change according to the head position and its attitude. Changing the viewpoint provides a sense of reality as if the blood vessels appear to be realistic such that the blood vessel is intuitively observed. In this study, the visibility of the HMD for vascular observation was investigated through an experiment to find a pseudo lesion embedded into the cerebral artery as shown in Fig. 1. The pseudo lesion was a cube with an edge length that was 0.9 times

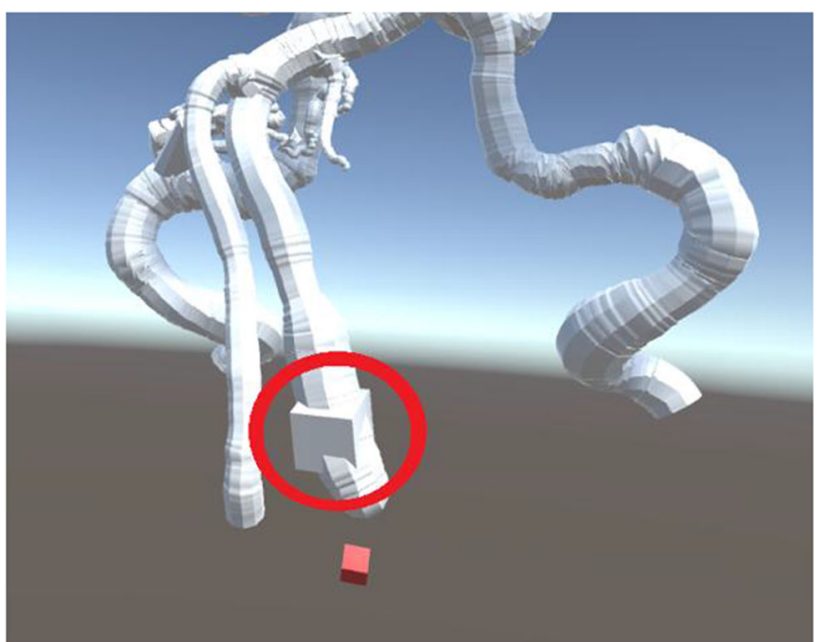

Fig. 1 Cerebral artery and embedded pseudo lesion (inside red circle)

larger than the blood vessel's diameter at the position. Pseudo lesions of three different sizes were used in this study to investigate the relevance of the lesion size to the visibility of the HMD. The edge length of the small, medium, and large pseudo lesions were approximately 2, 4, and $6 \mathrm{~mm}$, respectively. An examinee sought the pseudo lesion by translating, rotating, and scaling the vasculature. The time taken by the examinee to find the pseudo lesion was used as a measure of visibility. The examinees included 12 males and females in their twenties who were students in the department of science and engineering, not medicine. The same experiment was carried out using the 2D monitor instead of the HMD to compare the visibility of the HMD. Six examinees used the HMD before using the 2D monitor, while the other six examinees used the 2D monitor before using the HMD, considering the habituation effect. The display resolutions of the HMD and the $2 \mathrm{D}$ monitor were $1080 \times 1200$ pixels per eye and $1920 \times 1080$ pixels, respectively.

\section{Results}

Table 1 shows the average times taken for the 12 examinees to find the pseudo lesions of the three different sizes using the HMD and the 2D monitor. The time taken for the examinee to find the small-sized pseudo lesions using the HMD was significantly shorter than that using the $2 \mathrm{D}$ monitor $(p<0.05)$. The times taken for the examinee to find the pseudo lesions of the other two sizes using the HMD were not significantly different from those using the 2D monitor, suggesting that smaller objects are more visible when using the HMD than the 2D monitor. This might be because the stereoscopic representation of objects by the HMD enables depth recognition of objects.

\section{Conclusion}

This study investigated the visibility of the HMD for vascular observation through an experiment to find pseudo lesions of three different sizes embedded into the cerebral artery using the HMD. The time taken to find the small pseudo lesion using the HMD was

Table 1 Average times (mean $[\mathrm{s}] \pm$ standard deviation) taken by the 12 examinees to find the pseudo lesions of three different sizes using HMD and 2D monitor

\begin{tabular}{lccc}
\hline Size of lesion & Small size $^{\mathrm{a}}$ & Medium size & Large size \\
\hline HMD & $90.4 \pm 69.9$ & $67.5 \pm 87.0$ & $40.6 \pm 67.3$ \\
2D monitor & $218.7 \pm 192.0$ & $50.6 \pm 52.9$ & $54.0 \pm 51.6$ \\
\hline
\end{tabular}

${ }^{\mathrm{a}} p<0.05$ 
significantly shorter than that using the $2 \mathrm{D}$ monitor, suggesting that the stereoscopic representation is effective for observing small delicate objects such as the vasculature. This finding indicates that the HMD is also useful for the diagnosis of medical images. We aim to investigate the availability and superiority of the HMD to the 2D monitor in observing medical images for diagnosis and surgery under a more realistic clinical situation in the future.

\section{References}

[1] Shinohara T, Nishida Y, Nakasako N, Ogura N (2018) Vascular virtual handling in three-dimensional image for endovascular intervention assistance. International Journal of Computer Assisted Radiology and Surgery 13 (Suppl 1): S29-S30

[2] Shinohara T, Fukata K, Nakasako N (2019) Considering visibility of vascular virtual handling system for endovascular intervention assistance. International Journal of Computer Assisted Radiology and Surgery 14 (Suppl 1): S6-S7

\section{Dimensionality reduction for mass spectrometry imaging}

R. Ellis ${ }^{1}$, E. Ritcey ${ }^{1}$, R. Theriault ${ }^{1}$

${ }^{1}$ Queen's University, School of Computing, Kingston, Canada

Keywords Mass spectrometry, Digital Pathology, Image Processing, Breast Cancer

\section{Purpose}

This works aims to provide pathologists with real-time use of analytical chemistry in histopathologic studies, with specific application to breast cancer. Breast cancer is one of the most common forms of cancer among women in North America. Although lumpectomies are a generally successful procedure, the current absence of intraoperative margin analysis has resulted in a high re-excision rate for the surgery. Desorption electrospray ionization [1] is a method for Mass Spectrometry Imaging (MSI) that enables rapid analysis of tissue under ambient pressure and without causing damage to the tissue. A large challenge with the processing of MSI results is the sheer volume of data produced; an MSI scan of a $5 \mathrm{~mm} \times 5 \mathrm{~mm}$ tissue sample may have $500 \times 500 \times 1000$ entries. This challenges typical pre-processing techniques as well as the application of artificial intelligence techniques in a precise and efficient manner. As a result, the majority of MSI analysis for visualization has been univariate, investigating individual molecules as potential biomarkers for breast cancer. Recent work suggests application of multivariate techniques may assist in tissue identification in the large dimensionality space. Our goal was to implement dimensionality reduction techniques for MSI data to achieve reduced computation times while maintaining the quality of multivariate cancer versus benign tissue analysis. This could enable real-time clinical applications of multivariate techniques for breast cancer analysis and detection.

\section{Methods}

The data used for this study included 9 slices of thin excised breast tumor from 8 patients for which we had both complete histology and MSI data. The samples contained a mixture of cancerous and benign tissue. The sizes of the images ranged from $170 \times 122$ pixels to $190 \times 318$ pixels. Each pixel of each MSI image initially contained 1000 mass-charge abundances. Alignment of mass spectra across the images produced a single mass spectrum with mass-charge values consistent across all samples; this increased the dimension of each mass spectra to 3240 mass-charge abundances.

Seven reduction methods were extensively tested; the method we present had the best performance in terms of amount of reduction and computation time. The reduction began with decimation of the data by removing the $90 \%$ least abundant mass-charge values across each MSI acquisition. A graph clustering method in a proximal gradient descent framework was performed on the decimated data to segment the foreground, with background pixels removed. Non-negative matrix factorization (NMF) with Nonnegative Double Singular Value Decomposition (NNDSVD) initialization was performed on the foreground pixels. The 15 most relatively abundant mass-charge values from the top four components of the factorization were selected as important features for tissue subspace identification. The union of the important features of each sample formed the final feature space. The methods used in [2] were used to produce distance maps for the reduced data to show the presence of benign and malignant tissue in each image via multivariate analysis of the reduced data.

The same pipeline, without reduction, was also performed on the original, aligned data set referred to as the full data. Distance maps were produced from the foreground pixels by using the same multivariate techniques that were used on the reduced data. The distance maps were used as a control to compare the distance maps produced by the reduced data. Comparison of the distance maps were made quantitatively by calculating the mutual information (MI) similarity value between the cancer components of the distance maps.

\section{Results}

Our method produced a 94\% dimensionality reduction, reducing the number of mass-charge abundances from 3240 to 197 abundances. The reduction is visualized in Fig. 1. Mass-charge values greater than $640 \mathrm{Da}$ were removed in the reduction while ions ranging from $77 \mathrm{Da}$ to $638 \mathrm{Da}$ were selected as important features for tissue identification. On a laptop computer, this produced a $75 \%$ reduction in total computation time (Table 1) while maintaining quality of the distance maps (Fig. 2). Statistical comparison via MI of the distance methods are presented in Table 2. The MI value was on average 0.98 and did not decrease below 0.93 , suggesting nearly identical distance maps between full data and reduced data.

\section{Conclusion}

This work demonstrated a dimensionality reduction pipeline for MSI data that had very limited effects on multivariate analysis for tissue identification. The reduction-selected 197 mass-charge values were effective at distinguishing malignant from presumably benign tissue. This unsupervised reduction method identified ions in the range of $77 \mathrm{Da}$ to $638 \mathrm{Da}$ as being important features for detection. The range of selected mass-charge values suggests that fatty acids may play a part in distinguishing benign and malignant regions in excised breast tissue.

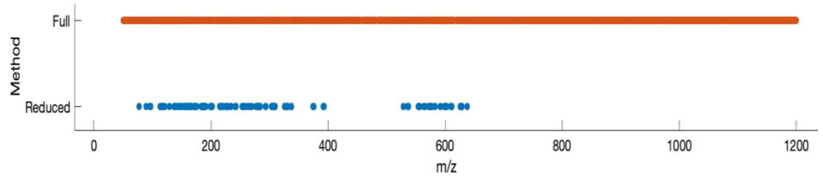

Fig. 1 The mass-charge values in the full dataset and the reduced dataset

Table 1 Computation times, in s, for creating the distance maps with the reduced data and with full data on a laptop computer

Computation time (s)

\begin{tabular}{lr}
\hline Reduced & 531 \\
Full & 2244 \\
\hline
\end{tabular}



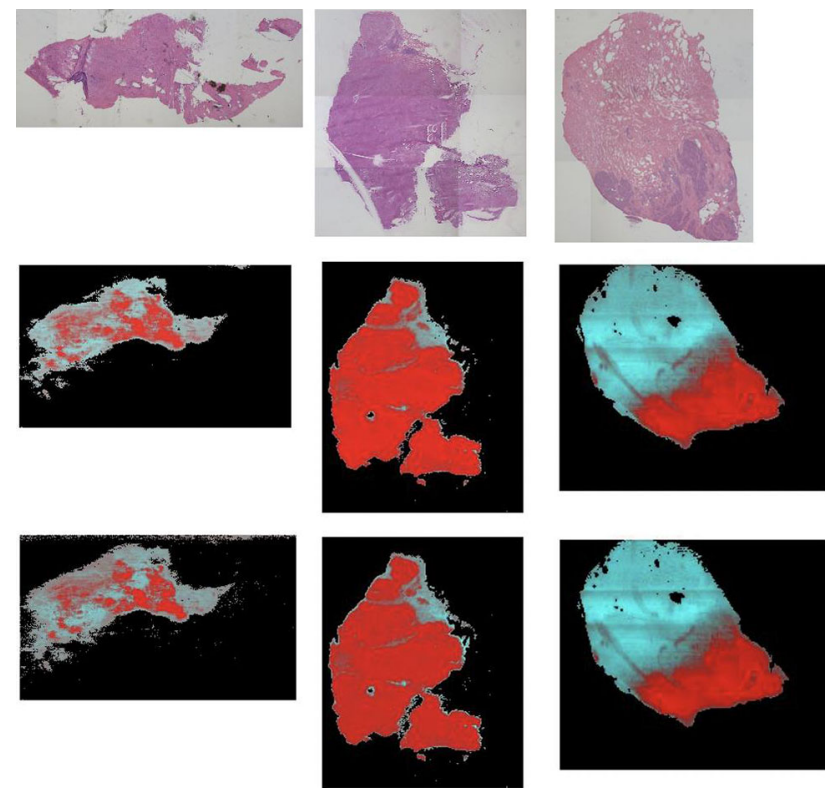

Fig. 2 For three tissue samples: the top row contains the histology, the middle row contains the distance maps produced from the full data, the bottom row contains the distance maps produced from the reduced data

Table 2 A summary of the mutual information (MI) similarity values, calculated for each tissue sample

\begin{tabular}{ll}
\hline & Mutual information \\
\hline Min & 0.9306 \\
Max & 0.9934 \\
Mean & 0.9742 \\
Median & 0.9832 \\
\hline
\end{tabular}

This work was conducted to investigate how dimensionality reduction might enable decreased run time and clinical application of MSI analysis. Future work could include using a larger sample size and investigation of how a mix of cancer types performs with dimensionally reduced data. This pipeline could be applied to other tissue types to further investigate its applicability in a clinical setting. The selected mass-charge values require further investigation, preferably with analytical chemistry techniques, to investigate their biological significance and potential for biomarker discovery. This method could be used to implement fast run times without loss of valuable information for tissue identification in breast cancer surgeries and to enable diagnosis in a clinical setting, which is yet to be seen in breast cancer surgery.

\section{References}

[1] Takats Z, Wiseman JM, Gologan B, Cooks RG: Mass spectrometry sampling under ambient conditions with desorption electrospray ionization. Science 306(5695):471-473, 2004.

[2] Theriault RL, Kaufmann M, Ren KYM, Varma S, Ellis RE. Metabolomics Patterns of Breast Cancer Tumors Using Mass Spectrometry Imaging. Int J Comput Assist Radiol Surg, accepted Dec. 2020.

\section{KHEOPS: Open source platform for storage and sharing of imaging biobanks}

J. Spaltenstein ${ }^{1}$, N. Roduit ${ }^{1}$, G. Pasquier ${ }^{1}$, N. van Dooren ${ }^{1}$, S. Cicciu ${ }^{1}$, O. Ratib ${ }^{1}$

${ }^{1}$ University of Geneva, Geneva, Switzerland

Keywords DICOM, Imaging Database, Open Source, Big Data

\section{Purpose}

To provide an Open-Source web-based platform for storage, communication, wide distribution, processing and analysis of large collections of medical images.

\section{Methods}

We developed an Open-Source framework to support open-access and research repositories of medical images geared specifically toward multi-centric collaborative initiatives featuring [1]:

- Fully DICOM-compliant image archive

- Open databank of medical images and multimodality imaging biomarkers

- Facilitate sharing of curated and consented data between research groups

- User-friendly cockpit for the management and sharing of images by users

- Imbedded HTML5 viewer (OHIF) as well as links to popular open-source viewers (OsiriX/Horos, Weasis)

- Open web-based APIs for developers of image analysis and data mining tools.

Its highly secured and flexible cockpit allows user to manage and exchange data through a simple paradigm of "shared albums" that consists of subset of data from multiple sources. The use of unique and revocable tokens allows users to manage and monitor the use of their shared data. A companion data anonymization gateway (KARNAK) was developed to allow template-based deidentification of imaging data before exporting them from clinical settings to a KHEOPS platform.

\section{Results}

This platform provides a flexible means for data sharing that gives access to Big Data repositories for machine learning and Radiomics. Its flexible cockpit allows user to easily handle and manage imaging collections and to exchange them through a simple concept of "shared albums". The use revocable tokens assigned by the owners of the data allows to easily share and monitor the use of their shared data. Special attention was given to facilitating the integration with existing institutional PACS as well as existing imaging databanks through a customizable anonymization gateway called KARNAK. Each institution can thereby define its own templates of data anonymization according to institutional rules and regulations defined by local ethic committees for each research study.

This platform was already downloaded and installed in several research centers and academic institutions worldwide. The platform was implemented in academic hospitals together with KARNAK anonymization gateway allowing researchers to gather cohorts of imaging data extracted from clinical PACS networks.

Using a web-based, zero-footprint open source viewer (OHIF viewer) the users can preview and analyze images from any imaging modality (including pathology images) on any platform including tablets and smartphones.

The platform architecture was specifically designed to allow image analysis and AI tools to be easily implemented by facilitating access to the image data through a simple interface allowing users to send an encrypted token of a given image set to a remote analysis process that can be hosted on a remote server to perform a specific 
image processing return numerical or image-based results. Several AI analysis tools developed by third party partners were tested through this interface allowing the AI analysis process to remain on a dedicated server with adequate processing and numerical capacity while images remained on the local KHEOPS server.

\section{Conclusion}

The web-based KHEOPS platform accessible on any device provides a cost-effective solution for securely hosting and sharing imaging data for research. Its user-friendly cockpit interface allows researchers to manage their own datasets and share them among selected community of participants. This platform is already being adopted by different national and international projects. It provides a cost-effective solution for securely hosting and sharing imaging data for research.

\section{References}

[1] Spaltenstein J, van Dooren N, Pasquier G, Roduit N, Brenet M, Pasquier G, Gibaud B, Mildenberger P; Ratib O (2020) A multicentric IT platform for storage and sharing of imagingbased radiation dosimetric data." Int J Comput Assist Radiol Surg 15(10): 1639-1643.

\section{A three-dimensional guidance based on fiber optical shape sensing, electromagnetic tracking and preoperative data for endovascular procedures}

S. Jäckle ${ }^{1}$, A. Lange ${ }^{1}$, V. García-Vázquez ${ }^{2}$, T. Eixmann ${ }^{3}$, F.

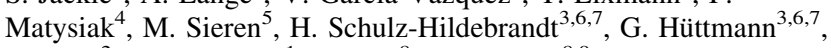
F. Ernst ${ }^{2}$, S. Heldmann ${ }^{1}$, T. Pätz ${ }^{8}$, T. Preusser ${ }^{8,9}$

${ }^{1}$ Fraunhofer MEVIS, Lübeck, Germany ${ }^{2}$ Universität zu Lübeck, Institute of Robotic and Cognitive Systems, Lübeck, Germany

${ }^{3}$ Universität zu Lübeck, Institute of Biomedical Optics, Lübeck, Germany ${ }^{4}$ University Hospital Schleswig-Holstein, Department of Surgery, Lübeck, Germany ${ }^{5}$ University Hospital SchleswigHolstein, Department for Radiology and Nuclear Medicine, Lübeck, Germany ${ }^{6}$ Medical Laser Lübeck GmbH, Lübeck, Germany ${ }^{7}$ German Center for Lung Research (DZL), Großhansdorf, Germany

${ }^{8}$ Fraunhofer MEVIS, Bremen, Germany ${ }^{9}$ Jacobs University, Bremen, Germany

Keywords Fiber optical shape sensing, Electromagnetic tracking, endovascular navigation, endovascular aneurysm repair

\section{Purpose}

In endovascular aortic repair (EVAR) procedures, two-dimensional (2D) fluoroscopy and conventional digital subtraction angiography together with the administration of contrast agent is the gold standard guidance for medical instruments inside the patient's body. These image modalities require X-ray exposure and the depth information is missing. Moreover, contrast agent is potentially kidney damaging for the patient. To overcome these drawbacks, a three-dimensional (3D) guidance based on tracking systems and preoperative data is introduced and evaluated using a realistic vessel phantom.

\section{Methods}

A model for obtaining the 3D shape position based on fiber optical shape sensing (FOSS), one electromagnetic (EM) sensor and a preoperative computed tomography (CT) scan was developed. This guidance method includes several steps. First the reconstructed shape is located in the preoperative CT scan with the EM sensor. As a result, the shape has the correct position and direction, but has the wrong rotation along the direction. Then, the shape is prealigned to the centerline path of the vessel to get an estimation of the correct rotation. Finally, the shape is registered to the vessel volume to obtain the accurate located shape. For evaluation, a stentgraft system (Endurant II AAA, Medtronic, Dublin, Ireland) was used. After dissembling it, a multicore fiber (FBGS Technologies GmbH) covered with a metallic capillary tube and one Aurora Micro 6 degree-offreedom EM sensor (length: $9 \mathrm{~mm}$, diameter: $0.8 \mathrm{~mm}$; Northern Digital Inc.) was integrated in the front part. Moreover, a custommade phantom with a $3 \mathrm{D}$ printed vascular system was created. An introducer sheath (Medtronic Sentrant ${ }^{\mathrm{TM}}$ Introducer Sheath, Medtronic, Dublin, Ireland) was inserted into the right common femoral artery of the phantom to facilitate the insertion of the stentgraft system. In the experiment, the stentgraft system was inserted into the aorta and pushed back approximately $2 \mathrm{~cm}$ steps between the measurements. At those five insertion depths data measurements of the tracking systems were done as well as image acquisitions for obtaining the ground truth.

Results

In the five different measurements we obtained average errors from 1.81 to $3.13 \mathrm{~mm}$, maximum errors from 3.21 to $5.46 \mathrm{~mm}$ and tip errors from 2.67 to $4.58 \mathrm{~mm}$ for the located shapes obtained with the guidance method. These errors are influenced both by the shape reconstruction (average errors from 0.51 to $1.26 \mathrm{~mm}$, maximum errors from 1.00 to $4.05 \mathrm{~mm}$ ) and the EM sensor accuracy $(1.13-1.50 \mathrm{~mm})$. Due to twist introduced by the introducer sheath the shape reconstruction errors are higher as in previous studies whereas the EM sensor errors are comparable. Despite the twisted shape, the errors of the located shape did not increase much for most measurements in comparison to the errors of the reconstructed shape and accurately located shapes were obtained with the introduced approach.

\section{Conclusion}

In this work, a 3D guidance method for a stentgraft system based on FOSS, the pose of one EM sensor and a preoperative CT scan was introduced and evaluated in an realistic experiment. The evaluation of the novel introduced guidance approach resulted in low errors and these results are promising for using this approach as guidance in EVAR procedures. Thus, future work will focus on further evaluations of the developed guidance in EVAR procedures. For this purpose, such an intervention will be conducted by navigating the stentgraft itself. This would facilitate the stentgraft placement in EVAR procedures.

\section{An end-to-end unsupervised affine and deformable registration framework for multi-structure medical image registration}

Z. Chen ${ }^{1}$, X. Gao ${ }^{1}$, G. Zheng ${ }^{1}$

${ }^{1}$ Shanghai Jiao Tong University, School of Biomedical Engineering, Shanghai, China

Keywords Unsupervised deep learning, Affine Image registration, Deformable Image registration, Multi-structure Registration

\section{Purpose}

Image registration is the process of determining a geometrical transformation that aligns coordinates in two or more images so that the attributes associated with different coordinates can be viewed and analyzed jointly. It plays an increasingly important role in medical image analysis due to its wide range of applications, including tracking relative positions of instruments and anatomy during surgery, integrating multimodal information like CT/MRI or atlas segmentation. Intensity based and handcrafted feature based methods are commonly used traditional registration methods, but these processes 
are inconvenient and time-consuming. Recently, unsupervised deep learning based methods show good performance and generalization ability in many registration tasks, while they still face a big challenge in dealing with multi-structure task $[1,2]$. On the other hand, global and local registration effects are hard to balance. Therefore we propose an end-to-end deep learning based unsupervised framework for simultaneously affine and deformable image registration and evaluate it on a $3 \mathrm{D}$ multi-structure registration task.

\section{Methods}

The proposed framework consists of two components: an affine network used to learn global transformation for coarse registration and a deformable network used to learn local deformation. The affine network is a deep convolutional network, which could learn 12 affine transformation parameters representing rotation, translation, scaling and shearing between the original fixed image and moving image. In order to enlarge receptive fields and get as much global information as possible, more convolutional layers and down-sampling operations are used in this network. Coarsely warped image is obtained by warping the moving image with the learned affine transformation. Next, a U-Net like deformable network is built to learn voxel-wise registration deformable field and then get the finally warped image. In the encoder stage, the network can learn and stack local features with different resolutions. And in the decoder stage, successive deconvolutional layers, convolutional layers and skip connections help the network use different resolution features to predict the displacement of each voxel. These two stages are then concatenated into an end-toend network. The detailed process is depicted in Fig. 1. The similarity metric function can be mean square error (MSE) or normalized crosscorrelation (NCC). And the smooth regularization function works on the gradients of deformable field. The overall loss function is designed as the weighed sum of the similarity between coarsely warped image and fixed image, smooth regularization and the similarity between finally warped image and the same fixed image, since every single part of the framework should be effective and the deformable field should be smooth enough to prevent distortions.

Experiments are designed to evaluate the performance of our framework. We include 30 hip CTs and evaluate on left femur, right femur and pelvis respectively. In details, for each structure, we have 20 train data, 9 test data and 1 fixed data. Before training, random affine transformation is done on train dataset for data augmentation, which could greatly improve the effectiveness of affine registration. Then in the training stage, all of input data pairs are centered first to ensure that they have overlaps, which is beneficial for the optimization process. Evaluation metrics used in our experiments includes Dice similarity coefficient, average surface distance (ASD) and residual mean square distance (RMS).

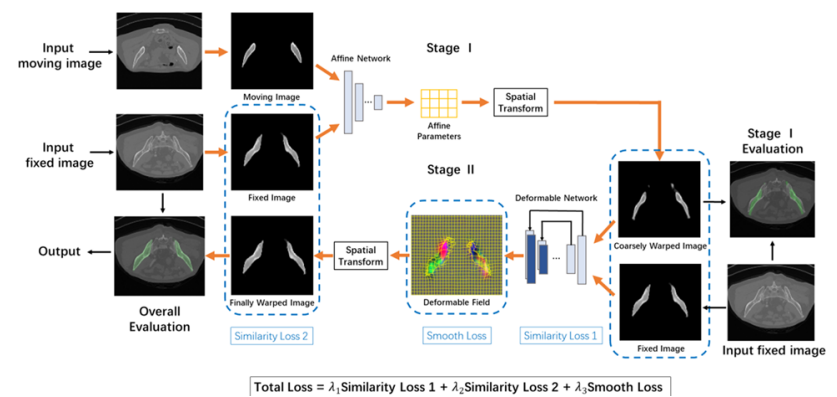

Fig. 1 Schematic representation of the proposed end-to-end framework for multi-structure registration. Three weighted losses are computed to optimize the parameters, specifically including the smooth loss of the deformable field, the other two similarity losses between the fixed Image and the warped Image of StageIand Stage II. Evaluation metrics including Dice, ASD, RMS
Table 1 Results of our experiments

\begin{tabular}{llrrr}
\hline & Methods & \multicolumn{1}{l}{ Left femur } & \multicolumn{1}{c}{ Right femur } & \multicolumn{1}{l}{ Pelvis } \\
\hline Dice & Before & $0.29 \pm 0.16$ & $0.34 \pm 0.20$ & $0.31 \pm 0.12$ \\
& Affine & $0.90 \pm 0.01$ & $0.89 \pm 0.01$ & $0.76 \pm 0.04$ \\
& Overall & $0.97 \pm 0.01$ & $0.96 \pm 0.01$ & $0.92 \pm 0.01$ \\
ASD & Before & $14.22 \pm 5.10$ & $13.15 \pm 5.79$ & $11.11 \pm 3.85$ \\
& Affine & $1.73 \pm 0.29$ & $1.82 \pm 0.22$ & $3.27 \pm 0.41$ \\
& Overall & $0.82 \pm 0.12$ & $1.05 \pm 0.20$ & $1.46 \pm 0.21$ \\
RMS & Before & $16.91 \pm 5.84$ & $15.87 \pm 6.63$ & $14.02 \pm 4.41$ \\
& Affine & $2.29 \pm 0.33$ & $2.40 \pm 0.25$ & $4.34 \pm 0.49$ \\
& Overall & $1.35 \pm 0.17$ & $1.57 \pm 0.25$ & $2.53 \pm 0.31$ \\
\hline
\end{tabular}

For each metric with each structure, the table shows three evaluation methods

\section{Results}

Table 1 provides an overview of quantitative results for this multistructure task in which for each evaluation metric, Before, Affine and Overall represent the corresponding values before registration, after only affine transformation and the overall performance by our proposed framework respectively. A mean overall Dice of $0.97 \pm 0.01$, $0.96 \pm 0.01$ and $0.92 \pm 0.01$ was found for left femur, right femur and pelvis respectively. Notably, an obvious improvement is achieved after the Affine transformation stage and the deformable part has a further approximate $10 \%$ increase of Dice over Affine. Meanwhile our method has an average $0.82 \pm 0.12 \mathrm{~mm}, 1.05 \pm 0.20 \mathrm{~mm}$ and $1.46 \pm 0.21 \mathrm{~mm}$ ASD. Furthermore, an average RMS of $1.35 \pm 0.17 \mathrm{~mm}, 1.57 \pm 0.25 \mathrm{~mm}, 2.53 \pm 0.31 \mathrm{~mm}$ was found for the three different structures.

\section{Conclusion}

Image registration is always an important subject in medical image analysis, but current registration methods still face challenges, such as multi-structure tasks and simultaneously global and local registration. We propose an unsupervised end-to-end affine and deformable registration framework to deal with different structure registration task. Experiments have demonstrated the effectiveness and generalization performance of our framework, which is mainly attributed to the framework can learn global and local deformation at the same time. And it has great potential to solve multi-structure registration problems. Even though we evaluated the framework on a 3D registration task, it can actually solve n-D registration task. Above all, our proposed framework has been proved to be effective to work as an efficient tool for multi-structure medical image registration.

\section{References}

[1] Jaderberg M, Simonyan K, Zisserman A (2015) Spatial transformer networks. Advances in neural information processing systems, 28, 2017-2025.

[2] Balakrishnan G, Zhao A, Sabuncu MR, Guttag J, Dalca AV (2019) Voxelmorph: a learning framework for deformable medical image registration. IEEE transactions on medical imaging, 38(8), 1788-1800. 


\section{COVID-19 lung infection and normal region segmentation from $\mathrm{CT}$ volumes using $\mathrm{FCN}$ with local and global spatial feature encoder}

\author{
M. Oda ${ }^{1,2}$, Y. Hayashi ${ }^{1}$, Y. Otake ${ }^{3,4}$, M. Hashimoto ${ }^{5}$, T. Akashi ${ }^{6}$, K. \\ Mori $^{1,2,4}$ \\ ${ }^{1}$ Nagoya University, Graduate School of Informatics, Nagoya, Japan \\ ${ }^{2}$ Nagoya University, Information and Communications, Nagoya, \\ Japan ${ }^{3}$ Nara Institute of Science and Technology, Graduate School \\ of Science and Technology, Nara, Japan ${ }^{4}$ National Institute \\ of Informatics, Research Center for Medical Bigdata, Tokyo, Japan \\ ${ }^{5}$ Keio University School of Medicine, Department of Radiology, \\ Tokyo, Japan ${ }^{6}$ Juntendo University, Department of Radiology, Tokyo, \\ Japan
}

Keywords COVID-19, segmentation, infection and normal regions, fully convolutional network

\section{Purpose}

This paper proposes an automated lung infection and normal regions segmentation method from lung CT volumes of COVID-19 cases. Novel coronavirus disease 2019 (COVID-19) spreads over the world causing the large number of infected patients and deaths. The total number of COVID-19 cases are more than 89 million in the world by January 10, 2021. To provide appropriate treatments to patients, rapid and accurate inspection method is required. Reverse transcriptase polymerase chain reaction testing (RT-PCR) is commonly used to diagnose COVID-19 cases. However, its sensitivity ranges from $42 \%$ to $71 \%$. In contrast, the sensitivity of chest CT image-based COVID19 diagnosis is reported as $97 \%$. Therefore, CT image-based diagnosis is promising to provide accurate diagnosis results. To provide more accurate and rapid diagnosis results to patients, computer aided diagnosis (CAD) system for viral pneumonia including COVID-19 is necessary. Such CAD system employs a segmentation method of infection (ground-glass opacity and consolidation) and normal regions in the lung. Segmentation results of them enables quantitative analysis of lung condition.

We propose an automated segmentation method of infection and normal regions from lung CT volumes of COVID-19 cases. Our original contribution is proposal of a 3D fully convolutional network (FCN) utilizing a local and global spatial feature encoder for accurate segmentation of infection regions that have various sizes and shapes. U-Net accurately segments lung normal regions because the shapes of the regions are similar among patients. However, infection regions have variations of sizes and shapes. Such variations are difficult to learn by U-Net or common segmentation FCNs. We utilize 3D versions of dilated convolutions [1] and dense pooling connections [2] in our 3D FCN to effectively encode spatial information to feature values. By using our $\mathrm{FCN}$, lung infection and normal regions are accurately segmented.

Methods

3D FCN model for segmentation

Encoder-decoder style FCNs such as U-Net are commonly used in segmentation. The encoder extracts feature values from images. Commonly, convolution kernels of small sizes and max pooling are used in encoders. However, use of small convolution kernels cause loss of global spatial information. Furthermore, max pooling also loses spatial information.

To reduce loss of such spatial information in the encoder, we introduce dilated convolutions [1] and dense pooling connections [2]. We use 3D dilated convolutions of different dilation rates that are connected parallelly (called as 3D dilated convolution block) to utilize local and global spatial information. Our 3D FCN has 3D U-Net like structure. We replace a 3D convolution layer in the encoder with the $3 \mathrm{D}$ dilated convolution block. We also replace 3D max poolings

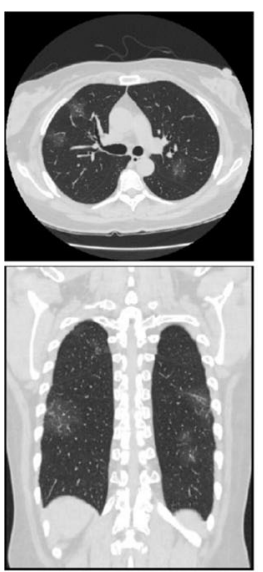

CT image
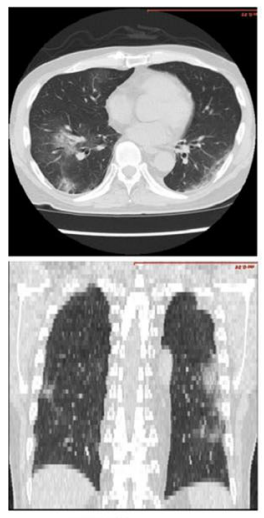

CT image

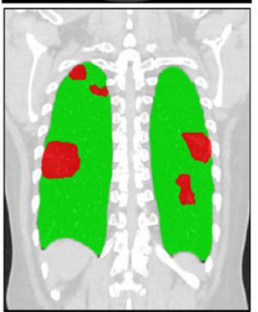

Ground truth
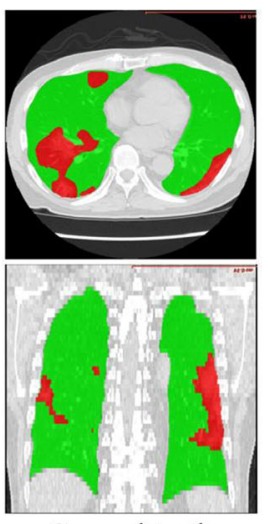

Ground truth

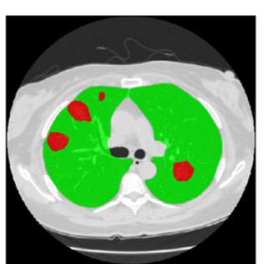

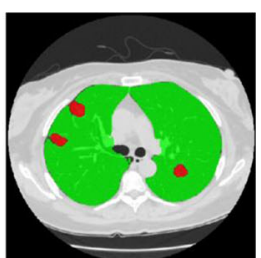

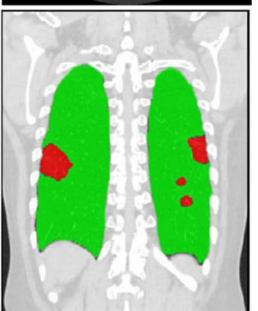

Segmentation result
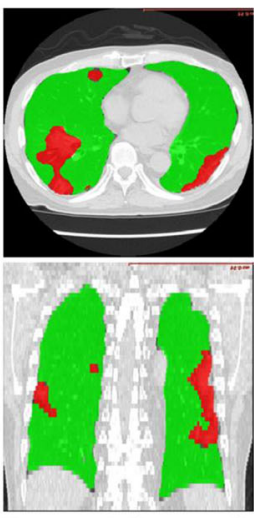

Segmentation result
Fig. 1 CT image, ground truth regions, and segmentation results by the proposed 3D FCN. Red and green regions are infection and normal regions. Top and bottom columns show axial and coronal slices

in the encoder with the $3 \mathrm{D}$ version of dense pooling connections to reduce loss of spatial information.

$3 D$ FCN training

We employ a 3D patch-based process to reduce GPU memory use. CT volumes for training are resized and clipped with $32 \times 32 \times 32$ voxel strides to make $64 \times 64 \times 64$ voxel patches. Data augmentations including translation, rotation, and elastic deformation are applied to the patches. Pairs of patches of CT volumes and the corresponding annotation volumes are used to train the $3 \mathrm{D} \mathrm{FCN}$. The annotation volume contains infection and normal regions in the lung, and outside body regions.

Segmentation

A CT volume for testing is resized and clipped to $64 \times 64 \times 64$ voxel patches. The patches are fed to the trained $3 \mathrm{D}$ FCN to obtain segmentation result patches. The patches are reconstructed to obtain a segmentation result in the same size as original CT volume.

Results

We applied the proposed segmentation method to $20 \mathrm{CT}$ volumes of COVID-19 patients. Specifications of the CT volumes are: image size is $512 \times 512$ pixels, slice number is 56 to 722 , pixel spacing is 0.63 to $0.78 \mathrm{~mm}$, and slice thickness is 1.00 to $5.00 \mathrm{~mm}$. Annotation volumes were checked by a radiologist. In the training of the 3D FCN, the minibatch size and training epoch number were set as 2 and 50. The generalized dice loss was used as a loss function. 
We evaluated segmentation performance in a fivefold cross validation. Dice coefficients of the proposed 3D FCN were 0.744 and 0.864 for infection and normal regions, respectively. As a comparison, we used 3D U-Net as the segmentation model and obtained 0.732 and 0.840 for infection and normal regions. Segmentation results by the proposed 3D FCN are shown in Fig. 1.

The proposed 3D FCN achieved higher segmentation accuracies compared to the 3D U-Net. Image features of infection regions that have large variations in their sizes and shapes are difficult to extract by the encoder in the 3D U-Net. The use of dilated convolutions and dense pooling connections improved feature extraction performance of the encoder by utilizing local and global spatial features. Therefore, we obtained higher segmentation accuracies by the proposed 3D FCN

\section{Conclusion}

We proposed an automated lung infection and normal regions segmentation method from CT volumes of COVID-19 cases. We developed a 3D FCN for segmentation that has an improved encoder for feature extraction from regions that have various sizes and shapes. $3 \mathrm{D}$ patch-based process was employed in our segmentation to reduce GPU memory use. In our experiments using $20 \mathrm{CT}$ volumes of COVID-19 patients, the proposed 3D FCN achieved higher segmentation accuracies compared to the 3D U-Net. Future work includes use of multiple patch sizes as the input of the 3D FCN and improvement of the decoder in the $3 \mathrm{D}$ FCN.

\section{References}

[1] Yu F, Koltun V (2016) Multi-scale context aggregation by dilated convolutions. International Conference on Learning Representations (ICLR)

[2] Playout C, Duval R, Cheriet F (2018) A multitask learning architecture for simultaneous segmentation of bright and red lesions in fundus images. MICCAI 11071:101-108

\section{CNN-based joint non-correspondence detection and registration of retinal optical coherence tomography images}

\author{
J. Andresen ${ }^{1}$, T. Kepp ${ }^{1}$, J. Ehrhardt ${ }^{1}$, C. von der Burchard ${ }^{2}$, \\ J. Roider ${ }^{2}$, H. Handels ${ }^{1}$ \\ ${ }^{1}$ University of Lübeck, Institute of Medical Informatics, Lübeck, \\ Germany ${ }^{2}$ Christian-Albrechts-University of Kiel, Department \\ of Ophthalmology, Kiel, Germany
}

Keywords Image registration, Non-correspondence detection, Optical coherence tomography, Macular degenerati

\section{Purpose}

Neovascular age-related macular degeneration (nAMD) is a retinal disease that causes vision loss due to abnormal blood vessel growth originating from the choroid. Injections of vascular endothelial growth factor inhibitors can slow down this process and even lead to amelioration of disease status. The monitoring of nAMD is performed using opical coherence tomography (OCT). An algorithm that registers OCT images of different time points could help to detect changes of pathologies and assist nAMD monitoring.

Due to the dynamic behaviour of the disease one patient's status may change drastically from one visit to another which leads to noncorresponding regions in the OCT images. Intra- and subretinal fluids (IRF and SRF), for example, are important biomarkers for nAMD progression that appear as dark spots on OCT images. Direct registration of pathological images can lead to severe registration errors since intensity differences are erroneously accounted for by image deformations. We therefore present a convolutional neural network $(\mathrm{CNN})$ for joint registration and non-correspondence detection in OCT images of nAMD patients. Our CNN registers intra-patient OCT images from different time points and simultaneously segments regions of missing correspondences. The network is trained using an image distance measure, a regularizer and the Mumford-Shah functional described in [1] as loss function and can handle a wide variation of deformations. The resulting segmentations of non-corresponding regions are shown to reflect pathological fluids inside the retina.

\section{Methods}

The proposed network for joint registration and non-correspondence detection is a y-shaped U-Net. Both decoders are connected with the encoder part of the network via skip connections. The first decoder outputs a vector field that is interpreted as a stationary velocity field. The matrix exponential of the vector field is calculated resulting in a diffeomorphic transformation that warps the baseline image to match the follow-up image. The second decoder outputs the segmentation of non-corresponding regions which are excluded from the distance computation during the registration. Input to the network are rigidly pre-registered, corresponding B-scans of baseline and follow-up OCT images.

Training is performed by minimizing a loss function composed of the mean squared error image distance evaluated on corresponding image regions (masking with the segmentation output), curvature regularization to smooth the deformation field and another regularizer favouring small segmentations with smooth boundaries according to the Mumford-Shah functional [1].

Weak supervision during the training of the registration task is introduced by an additional loss term that determines the Dice loss between retina segmentations of follow-up and baseline [2]. The segmentations were created by a medical expert who delineated the inner limiting membrane and the Bruch's membrane manually. No manual segmentations are required for application of the trained network.

The network is trained on 193 image pairs ( $9650 \mathrm{~B}$-scans) from 42 nAMD patients for which the acquisition times of baseline and follow-up image are no longer apart than five months. The images were taken with a Heidelberg Spectralis OCT device with a B-scan resolution of $496 \times 512$ pixels and cropped to the central 384 A-scans. The data is split on patient level into training, validation and test datasets. Five-fold crossvalidation is performed and for each fold the network is trained for 300 epochs with a batch size of 10, Adam optimization and a learning rate of $1 \mathrm{e}^{-4}$.

\section{Results}

The mean Dice score between the manual retina segmentations improved from 0.903 before to 0.961 after the baseline was transformed with the deformation output of the CNN. Our CNN is thus able to improve the spatial alignment between OCT-images of the same patient. The mean absolute error between the images improved from $7.75 \mathrm{e}^{-2}$ to $5.54 \mathrm{e}^{-2}$. To evaluate the segmentation output of the network, the mean absolute error between the registered images was calculated inside and outside the segmented regions. The error was $5.28 \mathrm{e}^{-2}$ in the corresponding regions and $3.61 \mathrm{e}^{-1}$ in the non-corresponding regions.

Figure 1 shows exemplar results of our registration and segmentation network. The figure shows that, depending on disease activity, the segmented regions either reflect growth or shrinkage of pathologies like retinal fluids. For patients with pathologies that are only visible in one of the images the segmentations directly align with these pathologies. This shows great potential of our network to be used for unsupervised pathology detection in retinal OCT images with the advantage of generating sharply delineated segmentations.

\section{Conclusion}

We presented the first results of a CNN designed to simultaneously register intra-patient OCT B-scans from different time points and 

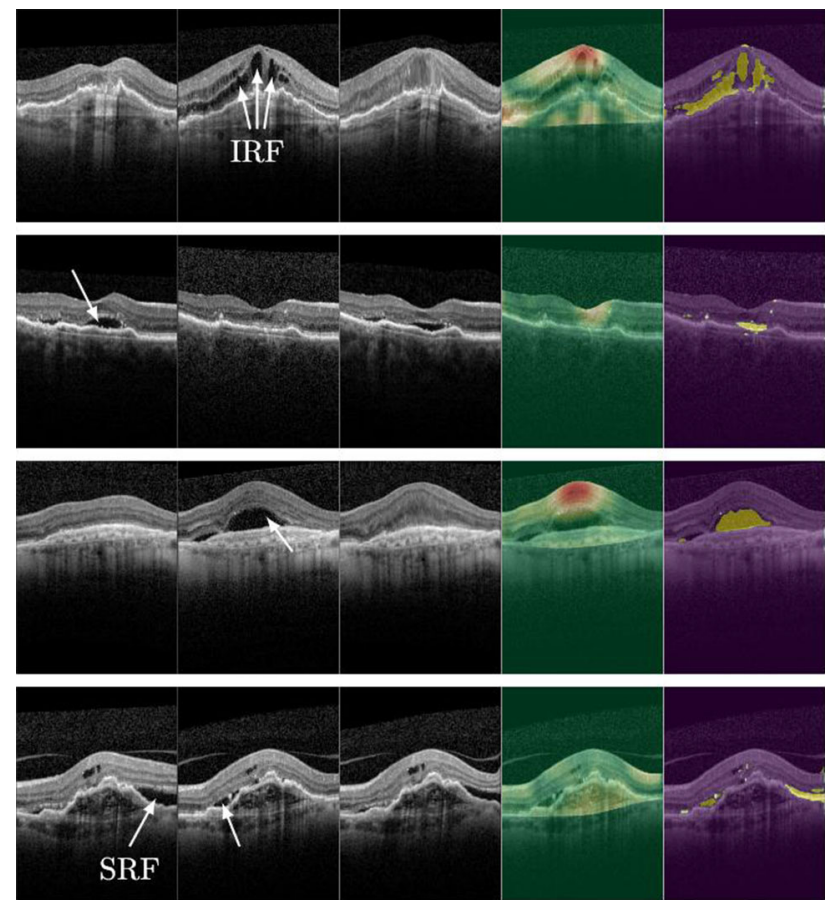

Fig. 1 Joint registration and non-correspondence detection results. Each row shows results for one patient. The first three columns show baseline B-scan, follow-up B-scan and registered baseline B-scan. The fourth column shows the follow-up image with the deformation field's magnitude overlaid (masked with manual retina segmentations). The fifth column shows the segmentation result of noncorresponding regions. Retinal fluids are exemplarily indicated with white arrows

segment regions where there is no correspondence between baseline and follow-up image. We show that CNN-based registration of OCT images profits from a Mumford-Shah segmentation of non-corresponding regions, as previously described in [1] for a variational registration approach. Our approach allows the end-to-end training of the registration-segmentation task. The trained network allows fast registration of OCT image slices and the resulting segmentations align with pathology changes. Our approach shows great potential for the monitoring of nAMD progression.

\section{References}

[1] Chen K, Derksen A, Heldmann S, Hallmann M and Berkels B (2015) Deformable image registration with automatic non-correspondence detection. International Conference on Scale Space and Variational Methods in Computer Vision: 360-371

[2] Hering A, Kuckertz S, Heldmann S, Heinrich M P (2019) Enhancing label-driven deep deformable image registration with local distance metrics for state-of-the-art cardiac motion tracking. Bildverarbeitung für die Medizin 2019: 309-314

\section{An ensemble of deep learning models to segment the optic disc in eye fundus images}

M. Ali ${ }^{1,2}$, M. Jabreel ${ }^{1}$, M. Abdel-Nasser ${ }^{1}$, A. Valls ${ }^{1}$, M. Baget Bernaldiz $^{2,3}$

${ }^{1}$ Universitat Rovira i Virgili-URV, Department of Computer Engineering and Mathematics, Tarragona, Spain ${ }^{2}$ Institut Investigacio Sanitaria Pere Virgili (IISPV), Tarragona, Spain ${ }^{3}$ University Hospital Sant Joan de Reus, Ophthalmology, Reus, Spain

Keywords Fundus image, optic disc segmentation, deep learning, opthalmology

\section{Purpose}

Optic disc (OD) assessment is essential for diagnosing glaucomatous damage and progression. Automated OD segmentation systems can handle a huge number of images and reduce human errors in screening. The focus of this work is to develop a reliable, accurate, and full automated system for segmenting OD in eye fundus images collected from Hospital Sant Joan de Reus (SJR) based on deep learning and ensemble techniques. The developed system has been also validated on two public datasets to demonstrate its reliability.

Methods

To develop a reliable and efficient OD segmentation system, we employ accurate individual deep learning-based segmentation models and an aggregation function to determine the largest consistency among the conflicting segmentation masks. As shown in Fig. 1, the input image is fed into three individual OD segmentation models, and then the resulting segmentation masks are aggregated to produce the final segmentation mask (final prediction).

In this study, we developed three accurate OD segmentation models based on state-of-the-art deep convolutional neural networks (CNNs) for image segmentation. Specifically, three networks, namely DoubleU-Net [1], DeepLabv3 + , and gated skip connections (GSCs) [2], are used here to build the individual OD segmentation models. We briefly introduce each model below:

DoubleU-Net includes two U-Net stacked architectures in sequence, two encoders, and two decoders. The first encoder and decoder are used in the lightweight model VGG-19 and the second is used in the U-Net network. DeepLabv3 + is an extension of DeepLabv3, which has a faster and stronger encoder-decoder network that can refine the semantic segmentation results, especially along with object boundaries. It can also extract feature vectors with Atrous convolution at various resolutions. GSCs is based on a U-shaped CNN which contains a contracting path and an expanding path, five encoder and five decoder blocks with a gated skip connection mechanism on the features received from the encoder layers.

In the aggregation step, we explored and tested various aggregation functions to construct the ensemble, finding that the median operator yields the best results. The SJR dataset contains 105 images:

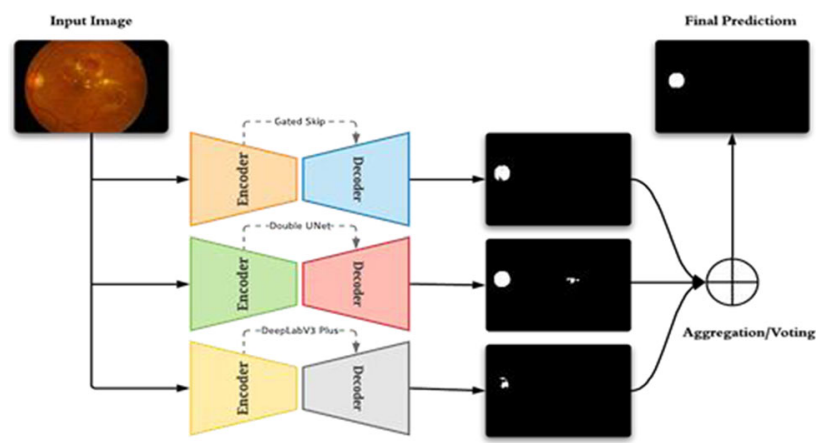

Fig. 1 Proposed system 
Table 1 Performance comparison in SJR dataset

\begin{tabular}{lllll}
\hline Method & \multicolumn{4}{l}{ Evaluation metrics } \\
\cline { 2 - 5 } & IOU & Dice & Recall & AUC \\
\hline U-Net & 0.924 & 0.919 & 0.875 & 0.937 \\
DeepLabV3+ & 0.941 & 0.938 & 0.901 & 0.95 \\
DoubleU-Net & 0.944 & 0.942 & 0.95 & 0.975 \\
GSCs & 0.951 & 0.949 & 0.929 & 0.965 \\
Proposed system & 0.955 & 0.954 & 0.952 & 0.976 \\
\hline
\end{tabular}

70 for training and 35 for testing. We trained the models on the SJR training set (100 epochs) and used the binary-cross entropy objective function with Adam optimizer (a learning rate of 0.001 , and a batch size of (2). The training set was augmented 20 times to improve the generalization of the OD segmentation models by applying horizontal and vertical flip, Hue, and rotation processes on the images.

Results

Table 1 presents the results of our system in terms of the intersectionover-union (IoU), Dice Score, Recall, and area under the curve (AUC). We achieved IoU and Dice scores of 0.955 and 0.954, respectively. Our system outperforms all compared models in all the evaluation metrics. Our system has been also evaluated on two publicly available datasets: IDRID (https://idrid.grand-challenge. org/Data/) and Drishti-GS (https://cvit.iiit.ac.in/projects/ mip/drishti-gs). With IDRID, it obtained IoU and Dice scores of 0.959 and 0.958 , respectively. It is remarkable that it achieved a 2.5 improvement on the IoU score on IDRID Leaderboard. With DrishtiGS dataset, our method achieved an IoU and Dice scores of 0.967 , which are better than the ones of the related work and standard medical image segmentation models, such as U-Net and DoubleUNet.

\section{Conclusion}

The proposed system produces accurate segmentation of the OD, which may reduce human errors for ophthalmologists in the detection of eye pathologies. We have demonstrated that the use of an ensemble of models improves the quality of the segmentation, reaching values above 0.95 in the usual indicators. In fact, the proposed model achieves accurate yet reliable segmentation results with the in-house dataset and two other public datasets (IoU > 0.95 and Dice $>0.96$ ). Acknowledgements

This work has been funded by the research project PI18/00169 from Instituto de Salud Carlos III \& FEDER funds. The University Rovira i Virgili also supports this work with project 2019PFR-B2-61.

\section{References}

[1] Jha D, Riegler M, Johansen D, Halvorsen P, Johansen H (2020) Doubleu-Net: A deep convolutional neural network for medical image segmentation. Proceedings of the IEEE conference on Computer Based Medical Systems (CBMS), pp. 558-564.

[2] Jabreel, M, Abdel-Nasser, M (2020). Promising crack segmentation method based on gated skip connection. Electronics Letters, 56(10), 493-495.
DRR to C-arm X-ray image translation with application to trauma surgery

M. Himstedt ${ }^{1}$, S. Häger ${ }^{1}$, S. Heldmann ${ }^{1}$, A. Petersik ${ }^{2}$, E. Zähringer ${ }^{2}$, H. Gottschling ${ }^{2}$, S. Manuel ${ }^{2}$, T. Lieth ${ }^{2}$, J. Modersitzki ${ }^{1}$

${ }^{1}$ Fraunhofer MEVIS, Lübeck, Germany ${ }^{2}$ Stryker Trauma \&

Extremities, SOMA Research, Schönkirchen, Germany

Keywords Deep learning, Generative adversarial network, Trauma surgery, Image guided interventions

\section{Purpose}

Deep neural networks (DNNs) leverage high-quality segmentation, registration as well as 2D-3D mapping for image-guided trauma surgery. However, the limited amount of readily available C-arm X-ray images (CXR) hampers the training of deep networks. Fortunately, the presence of numerous CT scans motivates the utilization of synthetic images for training. Thanks to their 3D representations CT scans enable to generate 2D digitally reconstructed radiographs (DRR) simulating various sensor and imaging pose settings. Yet these models learnt using DRRs are observed to perform worse compared to those being trained on real X-ray images due to their distinct imaging characteristics. Thus, an DRR-to-CXR translation is proposed allowing high-quality inference for subsequent tasks based on more realistic training data. This paper presents first results obtained with generative adversarial networks (GAN) incorporating the lack of corresponding CXRs and CT scans.

\section{Methods}

Image translation enables to transform images from a domain $\mathbf{A}$ into another domain B. Generative adversarial networks have

been demonstrated to achieve outstanding results for this task. As the input DRR a of domain A and CXR $\mathbf{b}$ of domain $\mathbf{B}$ are not paired, our approach makes use of CycleGAN. A generator $\mathrm{G}_{\mathrm{AB}}$ translates a to the CXR domain $\mathbf{B}$ and $\mathrm{G}_{\mathrm{BA}}$ vice versa with $\left|\mathrm{G}_{\mathrm{BA}}\left(\mathrm{G}_{\mathrm{AB}}(\mathbf{a})-\mathbf{a}\right)\right|$ and $\left|\mathrm{G}_{\mathrm{AB}}\left(\mathrm{G}_{\mathrm{BA}}(\mathbf{b})-\mathbf{b}\right)\right|$ being forward and backward cycle losses respectively. For a more detailed derivation of CycleGAN, the reader is referred to the original paper [1]. We make use of a U-Net-based generator architecture consisting of 9 downsampling blocks. The discriminator is fixed to a patchbased architecture classifying overlapping regions of $70 \times 70$ pixels. The input DRR images are preprocessed before being passed to the GAN. In particular, we apply an image complement to the raw intensities as well as a fixed circular cutout as it is common for CXRs (see Fig. 1). Omitting these steps results in a less stable training and a degraded synthesises quality. Due to the lack of corresponding DRRs and CXRs for evaluation we generate a paired dataset as follows. Real X-ray images are downsampled (factor 0.5) and upscaled (to original size) while applying a Gaussian filter $(\sigma=5)$. This allows to simulate reduced textures and lower resolutions, particularly for bone structures.

\section{Results}

For training our GAN we incorporate 100 samples with a size of $512 \times 512$ pixels for each domain which are expanded to $\mathrm{N}_{\mathrm{A}}=$ 968 DRRs and $N_{B}=968$ CXRs respectively by means of image augmentation (random rotations and translations).

The experiment paired utilizes a set of 100 paired samples being generated as detailed previously. Thanks to the paired images we are able to compare synthesized to References images. Within the second experiment unpaired our GAN is applied to an additional set of 100 samples. The output is evaluated by comparing input to reconstructed images obtained based on a full-cycle run. Quantitative examinations for the proposed methods are carried out using common metrics for GAN evaluations. In particular, we use MAE, SSIM and PSNR. Results are achieved using a modified PyTorch implementation of CycleGAN [1] (see Table 1). The goal of the first experiment is to examine the image differences 

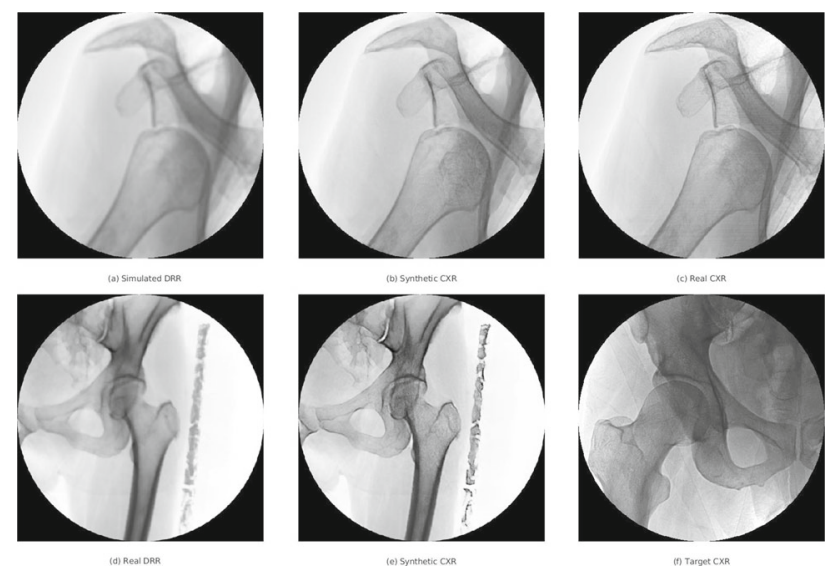

Fig. 1 Examples of synthetic CXR generation. a-c Refer to the paired experiment, $\mathbf{d}-\mathbf{f}$ to the unpaired experiment. a Is a simulated DRR, $\mathbf{d}$ a real DRR, b, e synthetic CXRs generated using our GAN, $\mathbf{c}$ is the real CXR for reference and $\mathbf{f}$ an example image of the CXR target domain. Note that there is no corresponding real CXR as reference for the unpaired dataset

Table 1 Experimental results for paired and unpaired experiments respectively

\begin{tabular}{lll}
\hline MAE \pm std & SSIM \pm std & PSNR \pm std \\
{$[0,255]$} & {$[0,1]$} & {$[\mathrm{db}]$} \\
\hline
\end{tabular}

\section{Paired}

$\mathbf{a}, \mathbf{b}$

$\mathrm{G}_{\mathrm{AB}}(\mathbf{a}), \mathbf{b}$

$2.245 \pm 0.710 \quad 0.833 \pm 0.054$

$27.858 \pm 3.660$

Unpaired
a, $\mathrm{G}_{\mathrm{AB}}(\mathbf{a})$
$0.722 \pm 0.591 \quad 0.953 \pm 0.043$
$38.723 \pm 4.847$
a, $\mathrm{G}_{\mathrm{BA}}\left(\mathrm{G}_{\mathrm{AB}}(\mathbf{a})\right)$
$0.697 \pm 0.1560 .987 \pm 0.005$
$40.537 \pm 1.742$
b, $\mathrm{G}_{\mathrm{BA}}(\mathbf{b})$
$0.942 \pm 0.4290 .954 \pm 0.029$
$36.964 \pm 2.936$
b, $\mathrm{G}_{\mathrm{AB}}\left(\mathrm{G}_{\mathrm{BA}}(\mathbf{b})\right)$
$0.781 \pm 0.221 \quad 0.976 \pm 0.020$
$38.984 \pm 1.449$

Values are means with $\pm 1 \sigma$ std. $\mathrm{G}_{\mathrm{AB}}$ (a) refers to the CXR synthesized based on DRR a. $\mathrm{G}_{\mathrm{AB}}\left(\mathrm{G}_{\mathrm{BA}}(\mathbf{b})\right)$ is the reconstructed image of $\mathbf{b}$ after a full cycle. Large values for SSIM/PSNR and low values for MAE are better

of paired raw images $(\mathbf{a}, \mathbf{b})$ compared to the synthesized $\mathrm{CXR} \mathrm{G}_{\mathrm{A}}$ в (a) and real CXR b. The second experiment additionally utilizes real DRRs, however, as the images $\mathbf{a}$ and $\mathbf{b}$ are unpaired, the evaluation is limited to measuring the reconstruction error given a and $\mathrm{G}_{\mathrm{BA}}\left(\mathrm{G}_{\mathrm{AB}}(\mathbf{a})\right)$ and vice versa for $\mathbf{b}$.

Based on our experimental evaluation, we can state that synthesized CXRs $\mathrm{G}_{\mathrm{AB}}$ (a) are closer to real CXRs b than DRRs a (paired: a, b vs. $\left.G_{A B}(\mathbf{a}), \mathbf{b}\right)$. The more detailed bone textures missing on DRRs become clearly visible on synthetic CXRs (see Fig. 1). Our GAN is able to generalize from various anatomical regions and acquisition poses. In addition to transferring contrast and intensity distributions it enables to reconstruct fine details of CXR images.

Conclusion

The presented approach allows to synthesize intraoperative CXRs from rendered DRRs given a limited amount of real unpaired data. This enables us to generate a large number of realistic paired samples for training networks addressing challenging tasks such as CXR segmentation as well as 2D$3 \mathrm{D}$ registration and mapping of bone structures which will be evaluated in our future work. Only a subset of the DRRs used for training contain fractures. For robustness, we will further expand this data ensuring that all putative bone fractures and anatomical variations are incorporated.

\section{Funding}

This work has been funded by the federal ministry of economic affairs and energy of Germany (BMWi, Funding number: 01MK20012Q)

\section{References}

[1] Zhu J-Y, Park T, Isola P, Efros AA (2017) Unpaired Image-toImage Translation using Cycle-Consistent Adversarial Networks, arXiv:1703.10593

\section{Thyroid nodule volume estimation and RF ablation using 3D matrix ultrasound: a phantom study}

T. Boers ${ }^{1}$, S. J. Braak ${ }^{2}$, M. Versluis ${ }^{3}$, S. Manohar ${ }^{1}$

${ }^{1}$ University of Twente, Multi-modality Medical Imaging, Enschede, Netherlands ${ }^{2}$ Ziekenhuisgroep Twente, Radiology, Enschede,

Netherlands ${ }^{3}$ University of Twente, Physics of Fluids, Enschede,

Netherlands

Keywords Radiofrequency Ablation, Ultrasound, 3D, Thyroid nodule

\section{Purpose}

The use of $2 \mathrm{D}$ ultrasound is well established for thyroid nodule assessment, however it offers a limited field of view that may lead to inaccurate thyroid nodule classification and reduced radiofrequency (RF) ablation effectiveness and an increase in complications. Real time 3D ultrasound offers an increased field of view by using three planes. This can potentially improve the clinical workflow, owing to assumed better volume estimation and improved nodule edge RF ablation. Here we assess the performance of a new 3D "matrix" transducer for thyroid nodule volume estimation and US-guided RF ablation, in comparison with a currently used 2D ultrasound approach. Methods

Twenty thyroid nodule phantoms underwent volume estimation and ablation guided by either one of two US-transducer types: a linear transducer (2D) and a real time 3D matrix (3D matrix) transducer. For volume estimation, the respective software-based calliper measurements of both transducer approaches were used, to calculate an ellipsoid volume approximating the volume of the nodule. Additionally, the 3D matrix transducer used its volume estimation tool. Thereafter, with one of two transducers, 10 phantoms were ablated. We used the transisthmic approach, evading the imaginary danger triangle that houses the recurrent laryngeal nerve and that has to remain clear of ablation, as well as the multiple overlapping shots technique in order to mimic the clinical ablation protocol closely. After ablation all 20 phantoms were cut into slices perpendicular to the RF ablation needle direction. The slices were photographed and analysed using Matlab running an in-house created pixel-based analysis algorithm. The algorithm calculates the volume of each nodule as well as the percentage of the nodule ablated and the ablated volume outside the nodule. The calculated total volume of each nodule was then used as the ground truth to which the US volume estimations were compared. Kruskal-Wallis tests were performed with a significance threshold of 0.05 . 


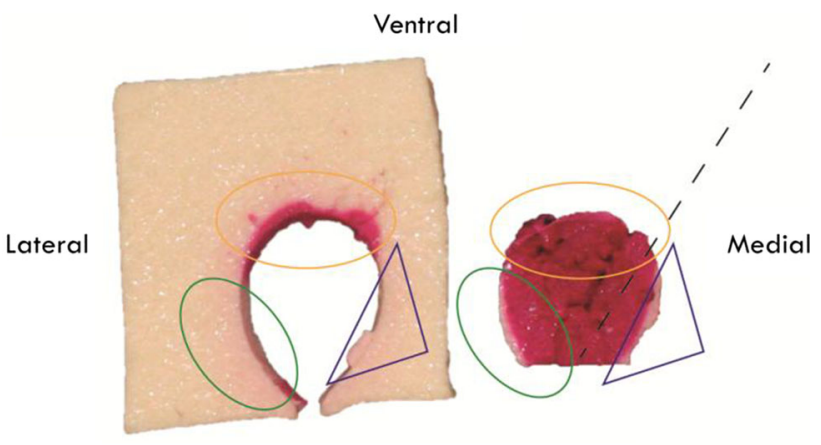

3D matrix

Dorsal

Fig. 1 Ablation results using 3D matrix guidance. The dashed line indicates the ablation needle orientation. The green circle indicates an area ablated closely to the edge, with a small rim of unablated tissue and minimal ablation outside the nodule. The blue triangle indicates the danger triangle with zero ablation outside the nodule. The yellow circle indicates an edge of the nodule fully ablated as well as the area outside of the nodule showing ablation

\section{Results}

We found that the matrix transducer volumes had a lower median difference with the ground truth compared to the standard 2D B-mode imaging (median differences $0.4 \mathrm{~mL}$ vs. $2.2 \mathrm{~mL}$ ). The 3D matrix guided ablation resulted in a similar ablation percentage when compared to the $2 \mathrm{D}$ approach $(76.7 \%$ vs. $80.8 \%)$. A 3D matrix guided ablation result of a nodule phantom can be seen in Figure 1. Although the 3D matrix and 2D guided ablations achieved higher ablation percentages, they also lead to ablated volumes outside the nodule (5.1 mL and $4.2 \mathrm{~mL}$, respectively). Of the locations outside the nodules some of the danger triangles showed minor ablations $(2 / 10$ and $4 / 10$, respectively). It is important to note that the $2 \mathrm{D}$ approach results were from a second batch of 10 phantoms wherein the median ablation percentages were found to be $13.4 \%$ higher than the first 10 phantoms, thus indicating a learning curve in the phantom ablation procedure.

\section{Conclusion}

This study has shown that 3D matrix transducer volume estimation is more accurate than the 2D transducer. Furthermore, 3D matrix transducer guidance during RF ablation is non-inferior to the current 2D transducer approach in ablating the nodule while staying clear of the nearby critical structures.

With the increased accuracy in volume estimation for the matrix transducer, diagnosis and follow-up results may aid in improved nodule classification. The longest-axis cut-off points in the TI-RADS protocols can be replaced with volume cut-off points that can be followed with more accuracy, potentially reducing the number of unnecessary biopsies.

The 3D matrix technology allows for simultaneous visualization of the perpendicular as well as the tangential plane relative to the needle giving the clinician a direct view of the needle position with respect to the nodule edge. This will potentially aid in ablating closer to the periphery of the nodule and thereby reducing vital peripheral nodular tissue ablation. However, this technology has to be developed further, due to the need for manual scrolling in the $3 \mathrm{D}$ matrix view and its potential learning curve.

\section{Development and evaluation of a 3D annotation software for interactive COVID-19 lesion segmentation in chest $\mathrm{CT}$}

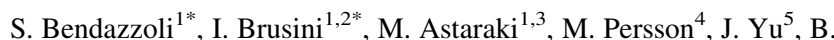
Connolly $^{5}$, S. Nyrén ${ }^{5}$, F. Strand ${ }^{3,6}$, Ö. Smedby ${ }^{1}$, C. Wang ${ }^{1 *}$

${ }^{1}$ Department of Biomedical Engineering and Health Systems, KTH Royal Institute of Technology, Stockholm, Sweden ${ }^{2}$ Department of Neurobiology, Care Sciences and Society, Karolinska Institute, Stockholm, Sweden ${ }^{3}$ Department of Oncology-Pathology, Karolinska University Hospital, Stockholm, Sweden ${ }^{4}$ Department of Physics, KTH Royal Institute of Technology, Stockholm, Sweden ${ }^{5}$

Department of Radiology, Karolinska Institute, Stockholm, Sweden ${ }^{6}$ Imaging and Physiology, Karolinska University Hospital, Stockholm, Sweden

*Contributed equally

Keywords: COVID-19, lesion segmentation, CT, semi-automatic

\section{Purpose}

We present a novel interactive tool for COVID-19 lesion segmentation, implemented within the software Mialab (http://mialab.org). The proposed workflow allows to segment both the lungs and the COVID19 lesions from input CT scans, by alternating automatic and manual steps within an intuitive user interface. This semi-automatic pipeline can thus speed up and aid the creation of ground-truth lesion masks, which may also be later employed for training automatic AI-based segmentation methods in a supervised fashion.

\section{Methods}

The present pipeline combines automatic segmentation methods with interactive manual editing and refinement, and it can be divided into four steps. First, a lung segmentation is produced by applying a shape model-based level set segmentation algorithm [1]. Secondly, the automatically segmented lung masks can be manually edited using a set of five interactive tools: a magnet tool, a brush tool, a splineinterpolation tool, a thin plate spline polyline tool and a smart clickand-drag tool. Additional information about the tools and their usage can be found at http://mialab.org/portfolio-item/covid-19/. A threshold-based level set segmentation algorithm is then applied to obtain a preliminary automatic segmentation of the COVID-19 lesions. Finally, the output lesion segmentations can undergo a final interactive editing step using the previously-mentioned tools.

The proposed software was tested on ten COVID-19 cases from the open access COVID-19 CT Lung and Infection Segmentation Dataset [2]. Six annotators were recruited and split into two groups according to their level of expertise: three radiologists (Expert Group) and three annotators with a technical background (Novice Group).

We computed the intra-class correlation coefficient (ICC model A, 1) - which evaluates the agreement in the lesion volumetric measures for the six annotators together, as well as for the Expert Group only and the Novice Group only. Moreover, for each of the ten segmented cases, we generated a Novice Group's and an Expert Group's consensus segmentation by performing voxel-wise majority voting within each group. We then computed the Dice coefficient, 95th percentile Hausdorff Distance and Jaccard coefficient between the consensus segmentations.

A further analysis of the spatial overlap between the lesions was then performed by calculating the generalized conformity index (GCI) both globally and within each annotator group.

The results from the enrolled annotators were then compared with the reference segmentation already provided as part of the dataset [2]. A Bland-Altman plot was used to investigate the volumetric agreement between the reference and the present annotators' segmentations. Moreover, their voxel-wise agreement was analyzed 
by computing the Dice coefficient, using the segmentation results from both all annotators together and for each separate expertise group.

\section{Results}

The agreement within and between annotator groups is presented in Table 1 . The highest volumetric agreement was obtained within the Expert Group, and the lowest within the Novice Group.

The two consensus segmentations obtained through majorityvoting showed a Dice coefficient of $0.845 \pm 0.113$ (mean \pm standard deviation), a 95th percentile Hausdorff distance of $44.714 \pm 21.5 \mathrm{~mm}$, and a Jaccard coefficient of $0.746 \pm 0.164$.

Furthermore, a GCI of $0.588 \pm 0.155$ was obtained for the global analysis, i.e. considering all six annotators together. On the other hand, the Expert group and Novice Group alone showed a GCI of $0.650 \pm 0.195$ and $0.545 \pm 0.149$, respectively.

Figure 1 shows the comparison of the consensus segmentations in some example slices from cases 2 and 10 of the dataset, which resulted, respectively, in a high and a low disagreement between the two groups.

The results obtained from the present annotators were also compared with the reference segmentation mask, obtaining a rather high agreement (see Figure 2). The biggest volume differences were observed between the reference with the Expert Group, rather than the Novice Group.

Furthermore, a slightly higher voxel-wise agreement was found between the reference segmentation and the Novice Group with a Dice coefficient of $0.719 \pm 0.129$, against $0.689 \pm 0.141$ obtained between the reference and the Expert Group.

The overall required annotation time resulted to be equal to $23 \pm 12$ min (mean \pm standard deviation across all raters and all 10 cases). The Expert and Novice Groups reported an annotation time of, respectively, $23 \pm 10 \mathrm{~min}$ and $22 \pm 14 \mathrm{~min}$.

\section{Conclusion}

The proposed semi-automatic tool led to promising results when tested by both expert radiologists and users with a technical background, suggesting the possibility of applying it in a vast range of applications within the scientific community. The pipeline was also shown to significantly speed up the segmentation process compared to a fully manual approach, thus helping to overcome the time efficiency issue of ground truth lesion mask creation.

Table 1 Summarized volume measurements across all annotators ("Global" column), across the annotators with a medical background ("Expert Group"), across the annotators with a technical background ("Novice Group"), as well as between the Expert and Novice groups ("Inter-group")

\begin{tabular}{lllll}
\hline Metric & Global & $\begin{array}{l}\text { Expert } \\
\text { group }\end{array}$ & $\begin{array}{l}\text { Novice } \\
\text { group }\end{array}$ & $\begin{array}{l}\text { Inter- } \\
\text { group }\end{array}$ \\
\hline $\begin{array}{l}\text { Mean Volume } \pm \\
\text { standard deviation } \\
\quad[\mathrm{mL}]\end{array}$ & $278.0 \pm 292.2$ & $295.6 \pm 318.8$ & $260.4 \pm 267.4$ & - \\
$\begin{array}{l}\text { Mean Absolute } \\
\quad \text { Volume Difference } \\
\quad \text { mL }]\end{array}$ & 54.7 & 38.4 & 48.9 & 73.0 \\
$\begin{array}{l}\text { Mean Relative } \\
\quad \text { Volume Difference }\end{array}$ & $23.0 \%$ & $18.4 \%$ & $17.7 \%$ & $24.5 \%$ \\
$\begin{array}{l}\text { ICC (A, 1) } \\
\quad(95 \% \text { confidence } \\
\text { interval) }\end{array}$ & $\begin{array}{c}0.899 \\
(0.785\end{array}$ & $\begin{array}{c}0.955 \\
(0.878\end{array}$ & $\begin{array}{c}0.886 \\
(0.707\end{array}$ & $\begin{array}{c}0.926 \\
(0.746\end{array}$ \\
\hline
\end{tabular}

The intra-class correlation coefficient (ICC) and its confidence interval were calculated using the ICC $(\mathrm{A}, 1)$ model
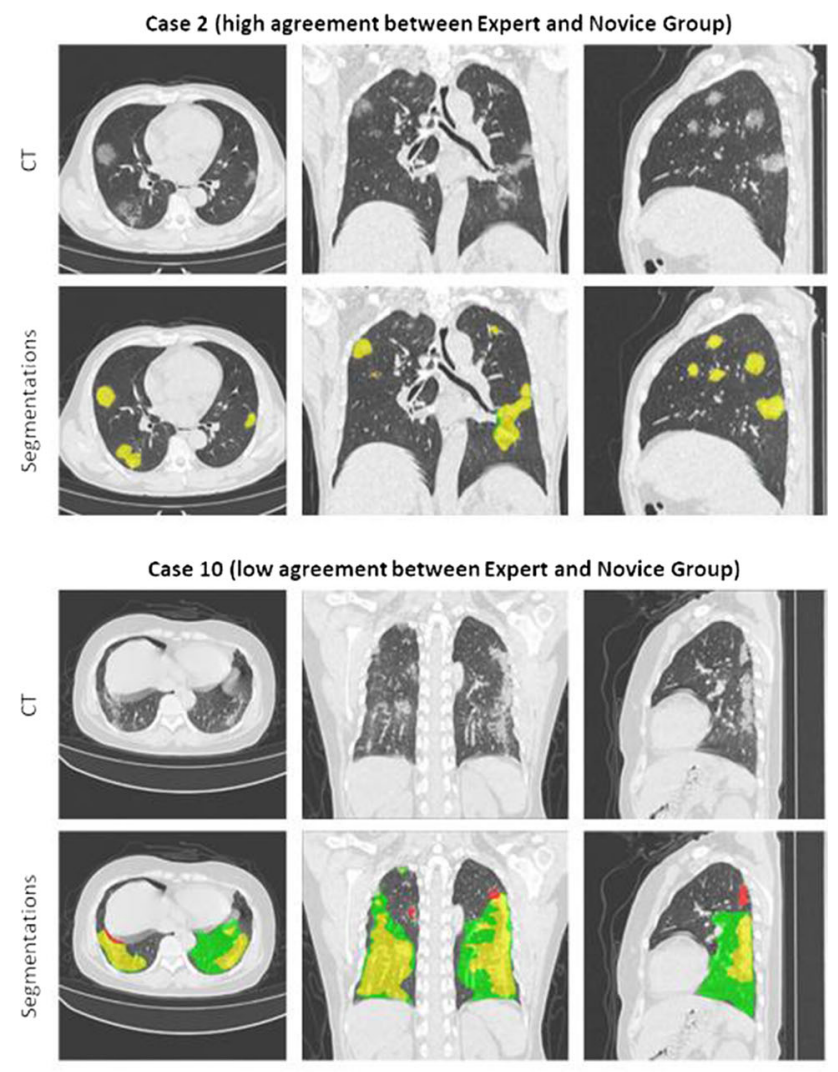

Legend

Lesions segmented by: Only Expert Group Both Groups

Fig. 1 Segmentation results in some example slices from all three image views, i.e. axial (left column), coronal (center) and sagittal (right). The results are shown for cases 2 (top) and 10 (bottom), which resulted, respectively, in a high and a low agreement between Expert and Novice Groups. For each case, both the original CT image slice and its overlaid segmentation masks are reported. Green areas correspond to regions labeled as lesions only in the Expert consensus segmentation, red areas are labeled as lesions only by the Novice Group consensus, while yellow regions correspond to an intersection between the two groups

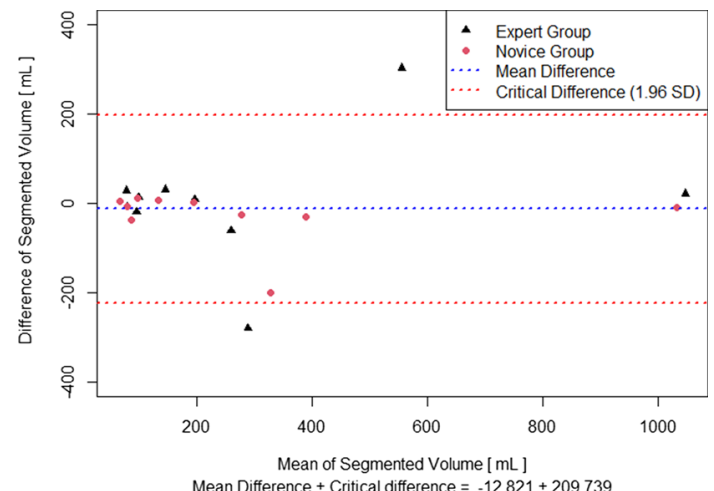

Fig. 2 Bland-Altman plot describing the agreement between the available reference segmentation and both the Expert Group (in black) and Novice Group (in red) segmentations 


\section{References}

[1] Leventon ME, Grimson WE, Faugeras O (2002) Statistical shape influence in geodesic active contours. In5th IEEE EMBS International Summer School on Biomedical Imaging, 15 (pp. 8-pp). IEEE.

[2] Ma J, Wang Y, An X, Ge C, Yu Z, Chen J, Zhu Q, Dong G, He J, He Z, Nie Z. Towards efficient covid-19 ct annotation: A benchmark for lung and infection segmentation. arXiv preprint arXiv:2004.12537. 2020 Apr 27.

\section{Dental implant planning, guide design and accuracy evaluation using free and open source software: an in vivo study}

\author{
D. Trikeriotis ${ }^{1}$, A. Theodoropoulou ${ }^{1}$, M. Mountricha ${ }^{1}$
}

${ }^{1}$ Private Practice, Athens, Greece

Keywords guide implantology, surgical guide implants, open source software, $3 \mathrm{~d}$ printing

\section{Purpose}

To explore the possibility of using and developing free and open source software in which the whole sequence of preparation of the working layout, implant planning, guide design and calculation of accuracy without postoperative computed tomography could be performed. Such a sequence could be acronymized as PIGA sequence derived from the initials of Preparation, Implant, Guide and Accuracy. The literature related to open-source solutions in dentistry appears limited [1]. In this context, the 3D Slicer software ( http://www.slicer.org) that is a free and open source software platform for medical image informatics, image processing, and threedimensional visualization was selected for evaluation.

Methods

27 partially edentulous patients seeking implant therapy, 9 men (mean age 50.5, range 40-68 years) and 18 women (mean age 49, range 21-62 years), underwent CBCT followed by a conventional impression and scan of the preoperative plaster cast with an intra-oral scanner (Medit i500). DICOM images and a 3D model were imported to 3D Slicer 4.11.0. Data was processed by using modules of the software package. Except for modules of import and addition, 18 more modules were used-each one in more than one of the 4 steps followed (Figure 1). Surgical simulation was harmonized with the sizes and lengths of components of the related guided system.

A total of 22 tooth supported and 10 tooth-mucosa supported surgical guides were 3D printed (PLA, da Vinci 1.0 Pro) and tested preoperatively as regards to seating on the teeth and tissues and availability of intermaxillary space. Master tubes were sterilized in an autoclave and surgical guides had a high-level disinfection by using ortho-phthalaldehyde $0.55 \%$ (exposure time $12-30 \mathrm{~min}$ at $\geq 20{ }^{\circ} \mathrm{C}$ ) according to the guidelines of CDC. 51 dental implants were placed (Xive, Dentsply) following the half-guided surgical protocol. After guided sleeve-on-drilling, the implants were placed freehand under direct bone visualization after incision.

The calculation of the placement accuracy in the RAS anatomical coordinate system was performed in 8 of the 27 patients after 12 implants were placed. These 8 cases met the following criteria: a) delayed dental implant placement into healed sockets and b) absence of changes in dentition during the time interval until restoration. At

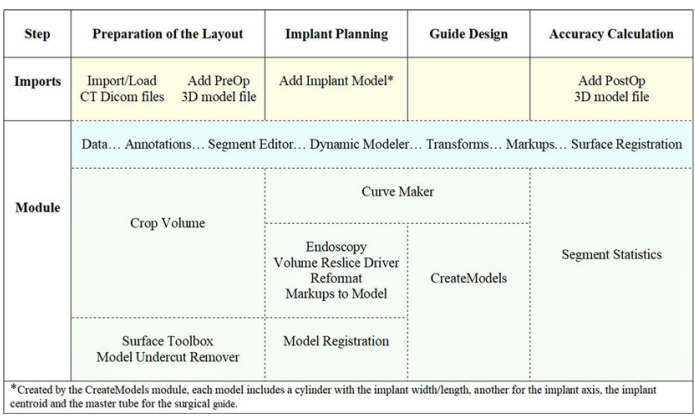

Fig. 1 Schematic workflow of 3D Slicer-based Preparation of the working layout, dental Implant planning, surgical Guide design and Accuracy calculation (PIGA sequence). Dashed lines emphasize the potential multiple use of modules during digital workflow

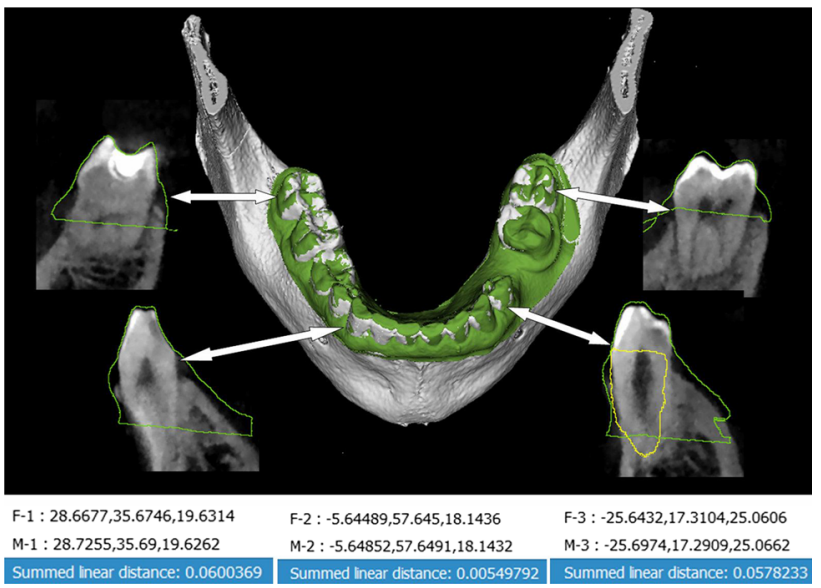

Fig. 2 The output model of a 3-point fiducial registration of the scanned imported model (green) to the CT anatomy model (white). At the bottom of the figure the deviations $(\mathrm{mm})$ of registered points at the sagittal, coronal and axial plane (RAS anatomical coordinate system) as seen in the Markups module

the time of restoration, an extra-oral scan (Medit i500) of the impression with implants identical to those placed and fixed to the transfer coping pickups was taken and imported into 3D Slicer for the calculation of accuracy. The registration process performed during the fourth step as well as the first step was monitored with numerical accuracy (Figure 2). MS excel software package was used for statistics.

\section{Results}

A digital workflow of implant planning, guide design and accuracy calculation without postoperative computed tomography was possible.

The mean linear deviation measured at the sagittal, coronal and axial plane found at the entry point was $1.03 \mathrm{~mm}$ (95\% CI: 0.662 to $1.4 \mathrm{~mm}), 0.47 \mathrm{~mm}(95 \% \mathrm{CI}: 0.3$ to $0.64 \mathrm{~mm})$ and $0.58 \mathrm{~mm}(95 \% \mathrm{CI}$ : 0.461 to $0.699 \mathrm{~mm})$, respectively and at the apex $1.1 \mathrm{~mm}(95 \% \mathrm{CI}$ : 0.744 to $1.46 \mathrm{~mm}), 1.05 \mathrm{~mm}$ (95\% CI: 0.722 to $1.38 \mathrm{~mm}$ ) and 
Table 1 Linear deviations at the sagittal, coronal and axial planes of the RAS anatomical coordinate system in relation to intra-oral orientation

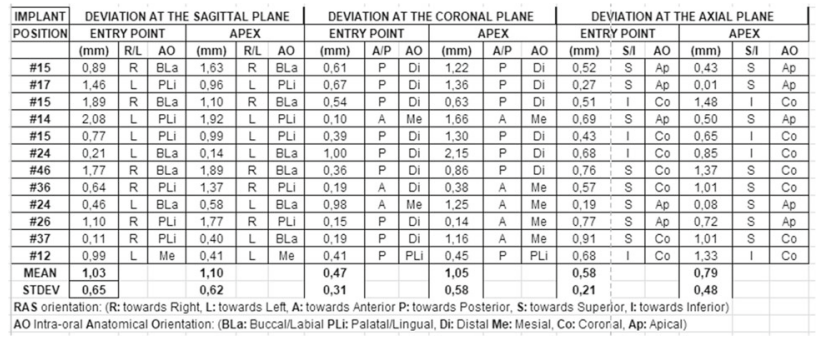

Table 2 Summed linear deviation at the entry point and the apex and angular deviation

SUMMED LINEAR DEVIATION ANGULAR DEVIATION

\begin{tabular}{|c|c|c|c|}
\hline \multirow{3}{*}{ IMPLANT POSITION } & \multicolumn{2}{|c|}{ SUMMED LINEAR DEVIATION } & \multirow{3}{*}{$\begin{array}{c}\text { ANGULAR DEVIATION } \\
\text { (degrees) }\end{array}$} \\
\hline & ENTRY POINT & APEX & \\
\hline & $(\mathrm{mm})$ & $(\mathrm{mm})$ & \\
\hline \#15 & 1,19 & 1,76 & 4,87 \\
\hline$\# 17$ & 1,62 & 1,65 & 6,22 \\
\hline$\# 15$ & 2,02 & 1,94 & 5,93 \\
\hline$\# 14$ & 2,19 & 2,58 & 8,19 \\
\hline$\# 15$ & 0,96 & 1,76 & 4,90 \\
\hline$\# 24$ & 1,22 & 2,31 & 6,98 \\
\hline \#46 & 1,95 & 2,48 & 4,64 \\
\hline$\# 36$ & 0,87 & 1,74 & 4,52 \\
\hline$\# 24$ & 1,09 & 1,38 & 1,13 \\
\hline$\# 26$ & 1,34 & 1,91 & 5,22 \\
\hline$\# 37$ & 0,93 & 1,58 & 6,58 \\
\hline$\# 12$ & 1,27 & 1,46 & 9,23 \\
\hline MEAN & 1,39 & 1,88 & 5,70 \\
\hline STDEV & 0,45 & 0,39 & 2,05 \\
\hline
\end{tabular}

$0.79 \mathrm{~mm}(95 \% \mathrm{CI}: 0.518$ to $1.06 \mathrm{~mm})$, respectively (Table 1$)$. The most frequent deviation was the "Distal" (9/12 implants) at the entry point and the "Coronal" (7/12 implants) at the apex.

The mean summed linear distance deviation at the entry point and the apex found was $1.39 \mathrm{~mm}(95 \% \mathrm{CI}: 1.13$ to $1.65 \mathrm{~mm})$ and $1.88 \mathrm{~mm}$ (95\% CI: 1.66 to $2.1 \mathrm{~mm})$, respectively. The mean angular deviation found was 5.7 degrees (95\% CI: 4.54 to 6.86 degrees) (Table 2).

\section{Conclusion}

The results regarding the accuracy of half-guided dental implant placement were consistent with literature [2]. 3D Slicer could be used in static computer-aided implant surgery as an alternative to commercial dental software. Although the presented workflow seems complex and time consuming there is, however, a great potential for improvements following a proper collaboration of software engineers and clinicians so that a Slicer-based IGA workflow can be generated in order for it to be clinically usable.

\section{References}

[1] Chruściel-Nogalska M, Smektała T, Tutak M, Sporniak-Tutak K, Olszewsk R (2017) Open-source software in dentistry: A systematic review. International Journal of Technology Assessment in Health Care 33(4):487-493

[2] Kühl S, Zürcher S, Mahid T, Müller-Gerbl M, Filippi A, Cattin P (2013) Accuracy of full guided versus half-guided implant surgery. Clin Oral Implants Res 24(7):763-769

\section{AI-based 3D-reconstruction of the colon from monoscopic colonoscopy videos-first results}

R. Hackner ${ }^{1}$, S. Walluscheck ${ }^{1,2}$, E. Lehmann ${ }^{3}$, M. Raithel ${ }^{4}$, V. Bruns $^{1}$, T. Wittenberg ${ }^{1,2}$

${ }^{1}$ Fraunhofer Institute for Integrated Circuits IIS, Erlangen, Germany ${ }^{2}$ University Erlangen, Erlangen, Germany ${ }^{3} \mathrm{E} \& \mathrm{~L}$ medical systems, Erlangen, Germany ${ }^{4}$ Malteser Waldkrankenhaus, Erlangen, Germany

Keywords colonoscopy, 3D-reconstruction, deep neural networks, depth maps

\section{Purpose}

For an improved image-based documentation of a screening colonoscopy it is desirable to determine which parts of the colon walls were seen during the examination and which sites potentially still need to be viewed. So-called panoramic images, being largescale, wallpaper-like images of the colon wall, are one possibility for such an enhanced documentation. Procedures already exist for the real-time creation of panoramic images of the urinary bladder floor or the oesophagus. Nevertheless, these approaches implicitly assume a simple and fixed geometry, such as a hemisphere or a tube. However, as the colon-in contrast to the bladder or the oesophagus-is an organ with a much more complex (partially tubular, partially flat, partially hemisphere) and dynamic geometry, these methods cannot be applied. In order to obtain adequate panoramas of the colon wall, a partial or fully $3 \mathrm{D}$ reconstruction of the colon geometry is necessary. Methods

Recently, deep-neural-network (DNN) based methods have been suggested and evaluated for various tasks in the field of image analysis in gastroscopy, such as automated adenoma and polyp detection, or differentiation of Barrett"s oesophagus. For the 3D reconstruction of the colon, or sections thereof, DNN-based methods can be applied that approximate depth maps in the form of point clouds from temporally sequential image pairs from colonoscopic grids. These depth maps and 3D- segments can then be assembled, registered and fused in successive steps [1].

For the first step of approximating depth maps, three deep neural networks with different architectures, namely "V-Net", "UNet", and "modified U-Net" (with a ResNet encoder) were constructed and evaluated. For training and validation, a set of 3600 successively acquired monoscopic image pairs (captured at $\mathrm{t}$ and $t+\Delta t$ ) from a synthetically created (digital) colon model (see details below) with known camera geometry were used. Using this data, each of the three networks was trained using 200 training epochs, and the achieved results were quantitatively evaluated against the known ground truth from the colon model. Additionally, the depth maps predicted for real endoscopy images were qualitatively evaluated, s. Fig. 1. Based on the intermediate results, the network that visually achieves the best results (U-Net with ResNet Encoder) was trained for 2.000 epochs on the 3.600 training image pairs and evaluated on 100 validation image pairs. For all
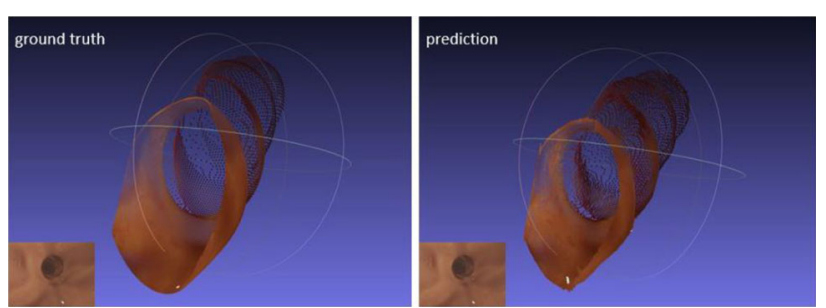

Fig. $13 D$ modelling of the depth of a colon section (left) and associated known ground truth from the digital data model (right) 
experiments an Adam optimizer with learning rate $r=1 \mathrm{e}-5$, a mean squared loss function and batch size $b=16$ was applied.

For the generation of sufficient synthetic colon data for the training of a deep neural network [2], a custom texture was designed using Blender. Voronoi textures were used to simulate vessel structures and fractal Perlin noise ("Musgrave texture") to obtain a slightly bumpy surface that allows a more natural impression. This also leads to more light scattering. The Musgrave texture originates from an algorithm initially designed to model landscapes. The mucus layer of the synthetic colon was modelled by using a BSDF glass shader with a refraction index that matches the one of water.

\section{Results}

For evaluation of the networks, the mean square error (MSE, L2Norm) and the mean absolute errors (MAE, L1-Norm) were employed to compare the resulting depth maps predicted by the DNN against the a priori-known ground-truth depth maps of the 100 test image pairs. The modified U-Net architecture with the ResNet Encoder reaches quantitative results of MSE $=0.000017$ and MAE $=0.001634$ over all 100 test data sets. Conclusion

The obtained results (see Fig. 1) suggest that an approximation of depth maps of the colon from monocular image pairs using a "modified U-Net" is possible in real time and leads to highly acceptable results, which can be used for a consecutive 3D colon reconstruction.

\section{References}

[1] Freedman D, Blau Y, Katzir L, Amit A, Shimshoni I, Veikherman D, Golany T, Gordon A, Corrado G, Matias Y (2020) Detecting Deficient Coverage in Colonoscopies. IEEE Trans. on Biomed. Eng. 39(11) pp. 3451-3462.

[2] Hackner R, Walluscheck S, Lehmann E, Bruns V, Eixelberger T, Wittenberg T (2021): A Geometric and Textural Model of the Colon as Ground Truth for Deep-Learning Based 3D-Reconstruction. Accepted, to appear. In: Ch. Palm, et al. (Eds.) Bildverarbeitung für die Medizin 2021. Informatik aktuell. Springer Vieweg, Wiesbaden.

\section{$\overline{\text { Image-based decision support system for catheter-based }}$ pulmonary valve replacement}

J. Yan ${ }^{1}$, S. Ching ${ }^{2}$, A. Wilson ${ }^{1}$, A. Lasso ${ }^{3}$, C. Herz ${ }^{2}$, A. Enquobahrie ${ }^{1}$, M. A. Jolley ${ }^{2}$, V. S. Arikatla ${ }^{1}$

${ }^{1}$ Kitware, Inc, Medical Computing Group, Carrboro, United States

${ }^{2}$ Children's Hospital of Philadelphia, Department of Anesthesiology and Critical Care Medicine, Philadelphia, United States ${ }^{3}$ Queen's University, Laboratory for Percutaneous Surgery, Kingston, Canada

Keywords pulmonary valve, finite element analysis, image processing, experimental validation

\section{Purpose}

Transcatheter Pulmonary Valve Replacement (TPVR) using self-expanding devices has evolved into a promising alternative to open surgical valve replacement in patients status post repair of Tetralogy of Fallot (TOF). However, determining patient candidacy and matching the optimal device to an individual patient remains challenging due to the complex, dynamic, and heterogeneous nature of the surgically repaired right ventricular outflow tract (RVOT). Current decision making is based on manually derived metrics, and laborious 3D printing based strategies, both of which erroneously assume that the vessel is rigid in the setting of device implant. Image based computational modeling holds promise for informing this process. We describe the ongoing development of an interactive image-based modeling tool, which now incorporates realistic finite element (FEM)based mechanics, to support the future identification of patients suitable for TPVR as well as selection of the best fit device for an individual patient.

\section{Methods}

We used custom open-source software to interactively place virtual models of transcatheter pulmonary valve (TCPV) into image based RVOT models. Existing CT scans of five sheep prior to and after the TCPV implantation in both end-systole and end-diastole were utilized for modeling [1]. Pre-implant images were imported into 3D Slicer [2] and the right ventricular (RV) and pulmonary artery (PA) blood pool are segmented using 3D threshold paint or grow cut algorithms as in our prior work [1]. The blood pool is then dilated to create a shell representation of the RV and PA (Fig. 1a). The segmented models are then imported into TetWild, to generate high-quality tetrahedral meshes. Finally, a finite element analysis of the device deployment process is conducted using FEBio, similar to prior modeling of self expanding devices performed using commercial packages. A frictionless contact model in FEBio, Sliding node-onfacet contact, is used to model the interaction between the vessels and the device. Two nonlinear hyperelastic models, Mooney-Rivlin and neo-Hookean, are adopted as the materials for the vessel tissues and the device, respectively. Prior to the deployment, the device is first compressed into a cylindrical catheter positioned coaxially. The compression is driven by the prescribed displacement on the outer surface of the catheter, and a frictional contact between the catheter and the device. To deploy the device into the vessel, a frictionless contact is defined between the device and the vessel, and two ends of the catheter are sequentially expanded as demonstrated in Fig. 1b, c. This approach can effectively avoid the sudden release of the high potential energy in the compressed device that could otherwise cause convergence issues in contact problems. However, when using this quasi-static analysis, the device needs to be fixed at certain mesh nodes to eliminate the rigid-body degree of freedom.

In order to assess device fit from resulting models, we developed quantitative metrics to evaluate whether a device is adequately sealed and properly anchored in both systole and diastole, while avoiding overcompression which could lead to device failure or erosion of the device into the vessel. Quantification is calculated both automatically and graphically. Deformation and stress measures of the device and vessel in the post-deployment state is shown in Figure 1b, c.

\section{Results}

We conducted FE analysis of a self-expanding TCPV replacement in five RVOT models. With the deployment strategy described above, FEBio was able to converge the challenging nonlinear contact

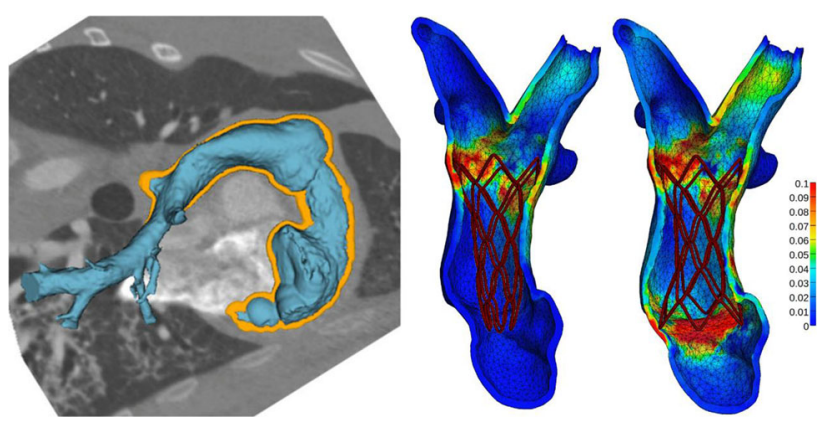

Fig. 1 Modeling Workflow. A) RVOT shell creation (orange) in 3D Slicer; B) FEM modeling of release of distal device; C) Release of both proximal and distal device. Color scale represents principle stress 
problems in all five cases, and generate visually realistic results. Fig. 1 shows the configurations of one systolic case with 41,000 and 73,000 tetrahedral elements in the vessel and the device, respectively. Penetration of device into the vessel wall in the converged state is about $25 \%$ of the wire diameter.

\section{Conclusion}

We have preliminarily demonstrated the feasibility of incorporating FEM into an evolving open-source based workflow for assessment of patient candidacy for TCPV replacement using self-expanding devices into native outflow tracts in TOF. This is significant considering the complex vessel geometries and the significant difference of mesh sizes and stiffness between the vessels and the device. We are now working to validate pre-implant modeling in comparison to actual device implants in the same surgical model. Evolution of this modeling may inform patient selection and optimal device for TCPV in congenital heart disease. In addition, the general framework is applicable to all self-expanding devices, such as many in clinical use for transcatheter aortic valve replacement.

\section{References}

[1] Jolley MA, Lasso A, Nam HH, Dinh PV, Scanlan AB, Nguyen AV, Ilina A, Morray B, Glatz AC, McGowan FX, Whitehead K, Dori Y, Gorman JH 3rd, Gorman RC, Fichtinger G, Gillespie MJ. (2019) Toward predictive modeling of catheter-based pulmonary valve replacement into native right ventricular outflow tracts. Catheter Cardiovasc Interv. 15;93(3):E143-E152.

[2] Fedorov A, Beichel R, Kalpathy-Cramer J, Finet J, FillionRobin C, Pujol S, Bauer C, Jennings D, Fennessy F, Sonka M, Buatti J, Aylward S, Miller JV, Pieper S, Kikinis R. (2012) 3D slicer as an image computing platform for the quantitative imaging network. Magn Reson Imaging. 30(9):1323-1341.

\section{FEM-based patient-individual modelling and simulation of knee joint motion}

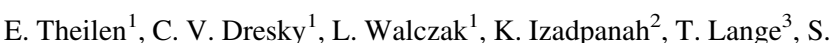
Bendak $^{2}$, D. Gehring ${ }^{4}$, J. Ketterer ${ }^{4}$, C. Huber ${ }^{5}$, J. Georgii ${ }^{1}$

${ }^{1}$ Fraunhofer Institute for Digital Medicine MEVIS, Bremen, Germany ${ }^{2}$ Medical Center-University of Freiburg, Department of Orthopedics and Trauma Surgery, Freiburg, Germany ${ }^{3}$ Medical CenterUniversity of Freiburg, Department of Radiology, Medical Physics, Freiburg, Germany ${ }^{4}$ University of Freiburg, Department of Sport and Sport Science, Freiburg, Germany ${ }^{5}$ Stryker Leibinger GmbH \& Co. KG, R\&D Robotics, Freiburg, Germany

Keywords biomechanics, knee joint, finite element method, patientspecific modelling

\section{Purpose}

The knowledge about the patient-specific knee joint kinematics is crucial for the decision on therapeutic measures in order to restore stability und functionality after a cruciate ligament rupture. To achieve best possible approximation to the patient's native pre-traumatic knee joint condition, a dynamic three-dimensional knee joint model based on the finite element method (FEM) is presented for the assessment of the individual biomechanics. In this approach the knee joint model is automatically derived from segmented MR images of the patient and from knee motion (flexion and rotation) captured by a motion tracking system (Vicon). The vision is to establish computerassisted ligament reconstruction planning in clinical routines.

Methods

For the development of the patient-specific knee model, MR images using either GRE or SPACE sequences with fat saturation are considered for the main knee structures: bones and the respective articular cartilages (i.e., femur, tibia, patella), menisci (i.e., medial and lateral meniscus), ligaments (i.e., anterior cruciate, posterior cruciate, medial collateral, lateral collateral and patellar ligament) and quadriceps tendon. All structures are manually segmented by clinical experts.

These developments have been inspired by the research investigated by [1]. In the present approach an automatic model generation has been conducted to fit into the clinical workflow. For a proper finite element (FE) model, thorough preprocessing of the structure segmentations is required to be able to neatly define contact conditions. The automatic procedure ensures reasonable geometries and that the structures do not overlap each other. Accordingly, a FE-model featuring tetrahedral meshes is automatically generated to fit into the open-source FEBio software [2] for non-linear implicit finite element simulations. In order to align the motion of flexion and rotation from the motion tracking system (Vicon) to the MR data of the patient's knee, a point-based registration has been performed. Thus, the captured patient-individual motion can be prescribed as boundary conditions to the femur while fixing the tibia.

For contact conditions of ligaments/tendon attached to bone at their proximal and distal ends, all ligament/tendon nodes in their intersection to the corresponding bone are detected automatically and defined to match the motion of the attached bone. Frictionless nonlinear contact conditions allow structures to slide across each other without penetration.

Furthermore, in the FE-model bones are considered to be rigid, so only their surface meshes are required. Articular cartilages are assumed to be linear, elastic and isotropic; and the menisci are modelled as linearly elastic and transversely isotropic material. The meniscal horn attachments on the tibial plateau are represented by linear springs. The behavior of the ligaments/tendon are described by transversely isotropic Mooney-Rivlin material using a fully-coupled formulation featuring initial strains defined from data available in literature.

Results

Figure 1 top row shows the result of the FE simulation of a knee flexion up to $20^{\circ}$ and subsequent external rotation up to $28^{\circ}$ of a healthy adult volunteer. The bottom row shows the stresses in the

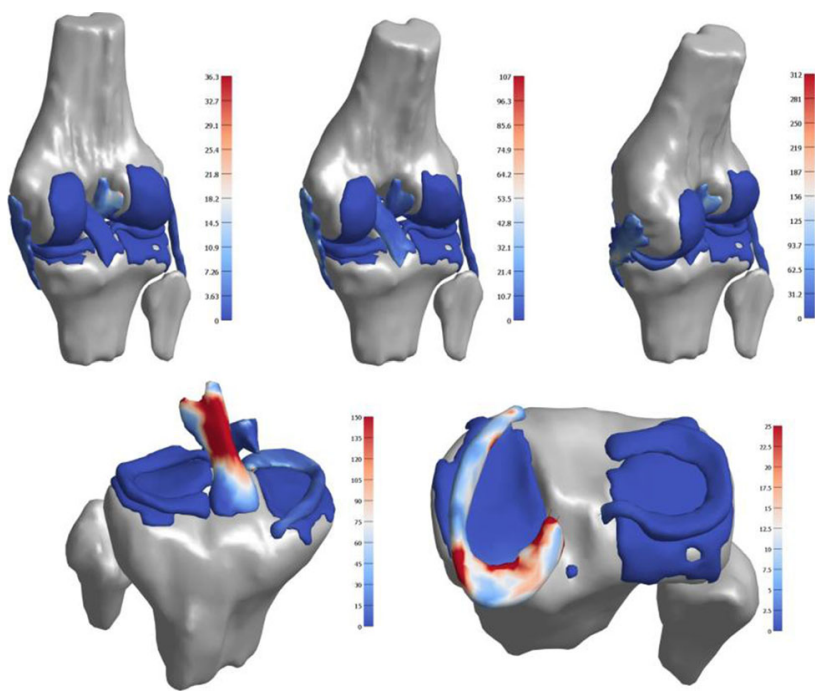

Fig. 1 FEM-simulation of a knee joint up to $20^{\circ}$ flexion with subsequent external rotation up to $28^{\circ}$ (top row) and the corresponding stresses $[\mathrm{MPa}]$ in ACL and medial meniscus at $20^{\circ}$ knee flexion and $28^{\circ}$ external rotation (bottom row) 
anterior cruciate ligament (ACL) and medial meniscus at the final position of $20^{\circ}$ flexion and $28^{\circ}$ external rotation.

The evaluation of the biomechanical dynamics, such as pressure distribution on the cartilage, stress curve in ligaments or the motion of the menisci can be used to quantify the extent of knee (in)stability and thus, derive patient-specific therapeutic measures in close cooperation with clinical experts.

Analyses and validation of simulation results against MR measurements at various flexion and rotation angles for 30 subjects (healthy and patients with isolated ACL rupture) as well as against cadaver data for 10 subjects, which will be analyzed dynamically in the lab, are in progress. Furthermore, kinematic quantification of different ACL-transplantation positions will be investigated with respect to clinical measures in operative care.

\section{Conclusion}

The finite element analyses based on automatic model generation from patient-specific MR image data and motion tracking of the knee joint are presented. This approach allows the quantification of the patient-individual dynamics, whose derived biomechanical parameters can be included in decisions on therapeutic treatment. Furthermore, this approach enables virtual planning on surgical interventions in advance for restoring best possible knee joint stability and functionality for each individual patient.

\section{References}

[1] Erdemir A (2016) Open Knee: Open Source Modeling and Simulation in Knee Biomechanics. Journal of Knee Surgery. 29(2):107-116.

[2] Maas SA, Ellis BJ, Ateshian GA, Weiss JA (2012) FEBio: Finite Elements for Biomechanics. Journal of Biomechanical Engineering. 134(1):011005.

\section{Automatic extraction of the knee joint's anatomical configuration in $3 D$}

E. Taghizadeh ${ }^{1,2}$, K. Izadpanah ${ }^{3}$, T. Lange ${ }^{4}$, P. Rovedo ${ }^{4}$, H. Meine ${ }^{1,2}$ ${ }^{1}$ University of Bremen, Informatics, Bremen, Germany ${ }^{2}$ Fraunofer MEVIS, Bremen, Germany ${ }^{3}$ Uniklinikum Freiburg, Orthopedics, Freiburg, Germany ${ }^{4}$ Uniklinikum Freiburg, Radiology, Freiburg, Germany

Keywords Patella luxation, Knee kinematics, Automatic 3D landmark detectio, Knee shape parameters

\section{Purpose}

Different factors can increase the probability of patellar luxation, including the bone shape the status of the ligaments and muscle activation. Diagnosing the individual risk factors for patella luxation in each patient might improve the treatment process. In clinical routines, 2D parameters are extracted manually for measuring the severity of these risk factors. In this work, we implemented an algorithm to automatically extract relevant patellofemoral joint parameters from 3D scans to facilitate diagnosis of recurrent patellar luxation.

\section{Methods}

The subjects' knees were scanned at the following flexion angles: extension (flexion angle $=0^{\circ}$ ), $15^{\circ}$ flexion, and $30^{\circ}$ flexion. At each flexion angle, loads of $0 \mathrm{~kg}$ and $5 \mathrm{~kg}$ were applied on the knee, so in total, each knee was scanned 6 times using a Siemens SPACE sequence in sagittal direction [1]. Currently, the dataset consists of scans of 14 patients and five healthy volunteers. For each subject, the image at extension without load (base image) was segmented manually.
Parameters extraction consists of the following steps:

1. Anatomical landmarks are automatically extracted on the femoral and patellar bones in the base image.

2. Each bone in other flexion/load cases is separately registered to the corresponding one in the base image, resulting in two transformation matrices per image, one for femur and one for patella.

3. The extracted femoral and patellar landmarks from step 1 are transferred to the bones on other loading situations, by applying respective transformations.

4. Kinematic parameters are computed for each flexion and load situation.

More details on each step follow.

Step 1. The landmarks and axes are extracted automatically on each segmented base image. These landmarks are explained in the following. The anatomical femoral axis (aFA) is computed using RANSAC as the best fit to the centerline of the femoral shaft. Transepicondylar axis (TEA) is the line connecting two epicondyles, which are the two mediolateral extremes of femoral bone when the image is aligned to have aFA parallel to Z-axis. The most distal landmarks on each condyle in the direction of aFA are extracted. The plane perpendicular to aFA with equal distance from these two landmarks defines the distal plane of femur. The transcondylar axis (TCA) is defined based on the center of two spheres that are fitted, using Hought transform, to two condylar surface joints. Posterior condylar landmarks are extracted as the extreme posterior points of each condyle in the anterio-posterior direction of femur. These two points define the posterior condylar axis of femur (PCA). Patellar reference point (PRP) is the patella geometry center of mass. The most distal point on patella in the direction of aFA is found. The most proximal landmark of patella is defined as the point on patellar surface with maximum distance from the most distal point. The axis connecting these points defines the proximal-distal axis of patella $\left(\mathrm{P}_{\mathrm{P}-\mathrm{D}}\right)$. The plane perpendicular to $\mathrm{P}_{\mathrm{P}-\mathrm{D}}$ containing PRP is intersected with patella. A contour is extracted from this intersection. The two points with maximum distance from each other on this contour define patellar mediolateral axis $\left(\mathrm{P}_{\mathrm{M}-\mathrm{L}}\right)$. Patellar anterioposterior axis is the cross product of $\mathrm{P}_{\mathrm{P}-\mathrm{D}}$ and $\mathrm{P}_{\mathrm{M}-\mathrm{L}}$.

Step 2. The femur (patella) bone segmentation in base image is dilated for $3 \mathrm{~mm}$. Then it is used as a mask to rigidly align the respective bone surface in each image to the base image based on normalized gradient fields. This registration gives us the transformation matrices for aligning each bone in the base position to the loaded/flexed situations.

Step 3. This transformation matrix is used to transfer the femoral (patellar) landmarks to the loaded image position.

Step 4. For quantifying the movement of patella w.r.t. femur under a specific flexion/load, a reference orthogonal coordinate system should be defined. We selected TCA as the mediolateral axis (M-L) and its center as the coordinate system origin $(\mathrm{O})$. Anteriorposterior axis of coordinate system (A-P) is the cross product of aFA and M-L axis, and the proximal-distal (P-D) axis is the cross product of A-P and M-L axes. Then the rotation and translation parameters of patella under load/flexion are computed in the reference coordinate system. In addition to these 6 parameters, we also defined lateralization as the translation of PRP projection on $\mathrm{M}-\mathrm{L}$ axis normalized by the TCA length, patella inclination as the angle between $\mathrm{P}_{\mathrm{M}-\mathrm{L}}$ and PCA, and patellar altitude as the distance of PRP from the distal plane of femur. 

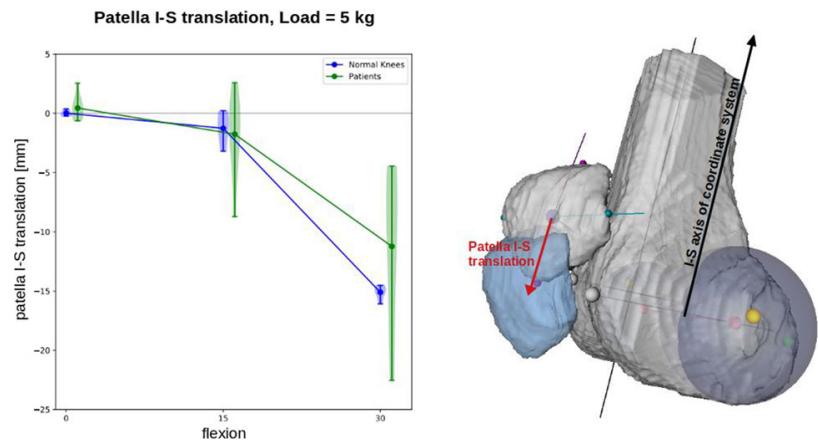

Fig. 1 Left: the violin plot of patella translation in the direction of I-S axis of the reference coordinate system, for healthy subjects (blue), and patients (green). Right: I-S translation is the magnitude of the red arrow. Here the knee of a patient when it is extended (gray bones) is shown. Patella movement is depicted for a $30^{\circ}$ flexion angle under a $5 \mathrm{~kg}$ load (blue patella)

\section{Results}

We analyzed seven shape parameters and nine kinematic parameters (times five for each flexed/loaded case). For almost all parameters, the variance of variables among patients were larger than the healthy cases. As an example, the patella translation along I-S axis of the reference coordinate system is shown in figure 1 , for different flexion angles when $5 \mathrm{~kg}$ load was applied.

\section{Conclusion}

We developed a pipeline to automatically extract clinically relevant landmarks and derived relevant parameters that represent shape and kinematics of the patellofemoral joint. Our results showed that the variance of parameters was larger among patients compared to healthy cases. This was expected for patients with patellar instability. Our work confirmed and, moreover, quantified these variations.

Our dataset consists of knee scans of patients with one or more risk factors, such as trochlea dysplasia and patella alta, as such the parameter distributions is not monomodal. Grouping patients based on the risk factors would facilitate better understanding of the patellar luxation and risk factors.

As part of our future work, we will increase the number of cases for reporting an in depth statistical analysis of kinematic parameters.

\section{References}

[1] Lange A, Taghizadeh E, Knowles B. R, Sudkamp N. P, Zaitsev M, Meine H, Izadpanah K (2019) Quantification of patellofemoral cartilage deformation and contact area changes in response to static loading via high-resolution MRI with prospective motion correction. Journal of Magnetic Resonance Imaging 50 (5): 1561-1570.

\section{The healthcare system of the future \\ D. Wilhelm ${ }^{1}$, D. Ostler ${ }^{2}$, C. Amato ${ }^{3}$, L. Bernhard ${ }^{2}$, O. Ratib $^{4}$ \\ ${ }^{1}$ Klinikum rechts der Isar, Technical University Munich, Surgery, München, Germany ${ }^{2}$ Research Group MITI, Klinikum rechts der Isar, Munich, Germany ${ }^{3}$ CannonDesign, Los Angeles, Germany \\ ${ }^{4}$ University of Geneva, Radiology, Geneva, Switzerland}

Keywords Robotics, Artificial Intelligence, Healthcare System, Transsectoral care

\section{Purpose}

The aim of this work is to assess upcoming technologies in respect to a future vision of the healthcare system and to predict potential developments for the upcoming period of time.

\section{Methods}

The results and thoughts which we present herein do originate from expert discussions and studies of the available literature. Also, aspects which were elaborated during the finalization of the "White paper on the Digitalization in Surgery" of the German society of surgeons are included. Still, the presented theses are speculative and not based on a fully scientific background.

\section{Results}

\section{Preventive medicine}

While in former times health was taken for granted and medical support was only requested in case of already present health related problems prevention of health is gaining attraction. Prevention affects both medical care, as well as private life in the form of healthy eating, weight control or daily sportive activities. Health trackers, wearables and smart devices already measure our heart rate, daily activity and stress level. The data together with detailed stratification of individual genomic and phenotypic profile, when analyzed by AI algorithms, can identify very early on health alterations which require preventive treatment.

\section{Data collection and storage}

It is assumed that the healthcare of the future will completely rely on a global data network and no longer be that personal and bilateral as it is today. Although we assume the contact of patients to their health providers will still represent the central pillar of healthcare it will not only be enabled by data and AI methods based on this, data will foster all healthcare related processes to the next level. A continuous personalized data flow thus forms the basis of care and will be fed with uncertified and certified information. This data flow is not restricted to the health-related environment, to medical departments or hospitals for example, but will be ubiquitous, will say, accompanies the patient everywhere and for his entire life. The capacity of collecting and curating large amount of data of each individual person can significantly empower the patients in their participation and awareness of their health status and assist them in adopting proper prevention and treatment strategies. The availability of comprehensive personal data of each individual person will allow for the generation of avatars or digital twins, that then can be used for modelling and evaluation of specific treatments regimes or even for preventive measures.

\section{Artificial intelligence}

AI represents the new panacea for the healthcare system and will expand from image dominated fields, e.g. in radiology and dermatology, to all other medical fields, as soon as data of adequate size and structure are available. AI will most likely be used to support our medical decision making and improve medical care by augmented approaches based on global knowledge and integration of large volume of data that single individual care-providers cannot achieve. AI already serves for physicians to re-become omniscient, as for example for defining the optimal cancer therapy and also for intraoperative decision making. In the near future, we can reasonably predict that $\mathrm{AI}$ will reduce the need for extreme specialization in healthcare and will allow for a more general and coordinated health approach. With constant feed-back from clinical results and patient reported outcomes the accuracy of AI-based decisions could further be improved and then provide a solid basis for augmented care approaches.

\section{Robotics}

Robots will become integral part of the healthcare system of the future. Whilst today they are predominantly used as master-slave systems in surgery, autonomous service and care robots (carebots) are supposed to play a much more relevant role in the coming decades. Autonomous nursing robots and automated devices are ideally suited to perform less demanding and repetitive tasks, saving time for the 
staff which then can be used for personal interaction with patients. But robotic technology will also contribute to the autonomy and flexibility of healthcare and we argue that self-navigating systems, capable of autonomously changing their position and adapt to specific frameworks and logistics, could bring a great advantage in optimizing treatment and monitoring procedures. The effective integration of robotics into surgical therapy will require a comprehensive standardization of surgical procedures and the support of a cognitive and highly "intelligent" environment. Accordingly, the cross linking of the different technologies inside the healthcare system will become a driving force and will foster the optimal usage and efficiency of implemented systems.

\section{The buildings of the healthcare system of the future}

We assume the healthcare system of the future will evolve from highly separated and specialized centers to a patient centered and health oriented omnipresent environment. This will span globally and include all areas, as for example home, working space or even mobile, while traveling. Only with smart capabilities that allow for a comprehensive monitoring of the patient's health status everywhere and at any time health problems can be detected the earliest possible. In this respect, the home and living environment of each individual will become a main area for less intrusive healthcare and wellbeing support systems mainly because this is where patients spend most of their time here and due to its privacy supporting sensitive examinations of vital signs. We also believe that there will be a seamless transition between home and hospital/medical departments, as care and diagnosis will already start in private areas.

\section{Conclusion}

The healthcare system of the future will be driven by technology, robots and artificial intelligence. Nevertheless, it will be more patient centred than it is today and patient satisfaction and maintaining "the human touch" will become core aspects. The maintenance of health and prevention of disease will serve as its main added value and will be enabled by a broad serration of all medical fields and the integration of non-medical environments.

\section{Innovation design for image guided therapies with disruption in mind-novel methodology for exploration, evaluation and impact generation}

\author{
M. Friebe ${ }^{1}$ \\ ${ }^{1}$ Otto-von-Guericke-University, Medical Faculty, HealthTec \\ Innovation Lab, Magdeburg, Germany
}

Keywords Healthcare Innovation, Clinical Translation, Biodesign, Purpose Launchpad

\section{Purpose}

A core problem of today's university based research-also in our dedicated area of Image Guided Therapies and Minimal-InvasiveProcedures-is that we are mainly focusing on creating research papers and that our research output is often judged by the amount of papers and citations. This value system encourages research activities on incremental innovations that come with a relatively low risk of failure and that can lead to a publishable result in a relatively short time. Disruptions can be defined as technologies or processes that lead to a significant $(>10 \mathrm{x})$ quality improvement or reduction in cost. Many published results today show improvements of some percent points in a very narrowly defined area. We do not want to stop these improvements and we do believe that these developments come with a value. They are however not typically leading to completely novel approaches and are not typically solving the huge challenges that we are facing in health delivery (chronic diseases, inequalities, urbanrural delivery differences, increasing cost, healthcare instead of sickcare, and many more). Exponential technologies (AI, Big Data,
Deep Learning, advanced sensors/wearables, robotics) will cause a paradigm shift in healthcare delivery and eventually not only lead to different development value propositions, but will also see different roles for different stakeholders (e.g. an empowered patient).

Healthcare is in need of INNOVATION and technologies for the digital transformation will cause unpredictable, but most likely very significant workflow changes in healthcare delivery and associated business models and will also need to follow different development criteria and value propositions $[1,2]$.

\section{Methods}

Our lab follows a novel approach for innovation generation with a clear focus on identifying and validating disruptive approaches based on UNMET CLINICAL NEEDS. We have also created a 5 ECTS interdisciplinary (students from Engineering, Natural Sciences, Medicine) lecture that provides attendees with much needed $21 \mathrm{st}$ century skills to address future health challenges and innovation needs. For this novel Innovation approach we combined several innovation methodologies including the BIODESIGN and the PURPOSE LAUNCHPAD teachings. The job of the researchers (and students) is to invest a lot of their initial work in identifying the UNMET CLINICAL NEEDS, analyze and properly understand the underlying problems, ideate possible solutions, and validate the problem/solution with the stakeholders. A focus on future developments needs to be based on patient empathy around an UNMET CLINICAL NEED. The role of university based research cannot stop with the publication of a paper. It needs to primarily focus on providing a value to the patient and society. For that we need to teach our researchers the basics of translation, which includes economic issues, future forecasting, and actual implementation of a validated idea/concept (see Figs. 1, 2).

To develop patient/society related products and concepts we will need to understand the underlying clinical problem including the

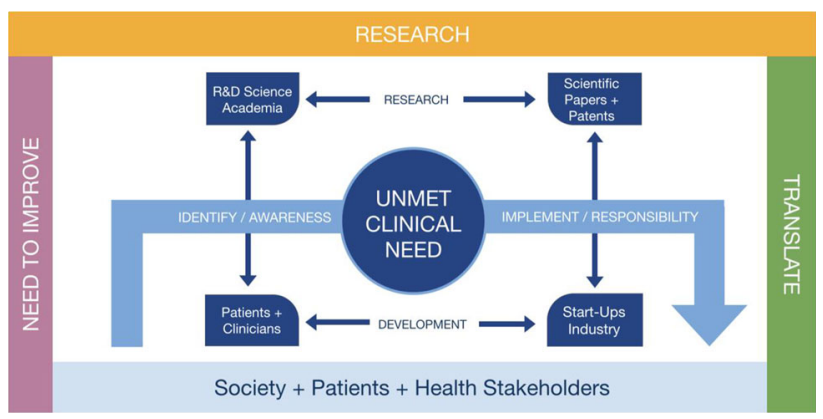

Fig. 1 Development approach that starts with an UNMET CLINICAL NEED (the need to improve) and includes research as well as translation to actually create a benefit for the patient and society

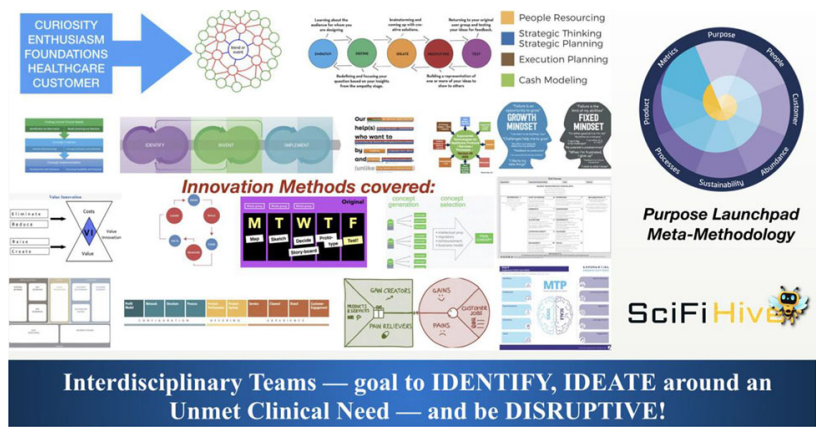

Fig. 2 Interdisciplinary Teams-goal to IDENTIFY, IDEATE around an Unmet Clinical Need-and be DISRUPTIVE 
current ways of diagnosing and treating it in combination with technological forecasting capabilities and an economic understanding. We have designed a new approach based on an adapted Stanford BIODESIGN approach of IDENTIFYING UNMET CLINICAL NEEDS, IDEATE solutions for these needs, iterate with the stakeholders and subsequently work on IMPLEMENTATION as start-up companies. This is combined with other VALIDATION models and experiments (PURPOSE LAUNCHPAD methodology, Alexander Osterwalder's Value and Business Model Canvas, Exponential Canvas and Massive Transformative Purpose approaches) to properly understand the problem, the future opportunities and to create and validate ideas through Minimal Viable Prototypes.

Research in general is future oriented and in the particular field of HEALTHTEC INNOVATION should consider the effects and possibilities of new technologies and their effect and needs to work on TRANSLATING the results of research into clinical practice. As INNOVATION is a combination of technical solutions with identifying the clinical need it is also necessary to understand regulatory issues and health-economics. The Future of healthcare will be data driven and will combine personal health records with a more comprehensive data and management structure requiring integrated devices, forensic information, advanced learning tools, and many more to eventually provide a digital twin that will then be able to manage, predict, and recommend personalized health related procedures and actions.

We believe that we need to combine technological depth with more broader skills and a novel approach of exploring and validating clinical problems. The paper will present the details of the innovation approach that includes:

- several future oriented innovation tools;

- the need to work in interdisciplinary innovation teams in an exploration and validation phase;

- the need to think disruptive with a goal significantly improve the quality, user and clinical experience, and dramatically reduce the potential cost of device or process;

- uses the PURPOSE LAUNCHPAD meta-methodology for Exploration (Biodesign: Identify), Evaluation (Biodesign: Ideate and partly Implement), and subsequent Impact Generation (Biodesign: Implement), Ethics and Exponential Canvas, Massive Transformative Purpose, Blue Ocean, Innovation Segment Concept, Exponential Technology Canvas, Value Proposition and Business Model Canvas, and associated entrepreneurial tools;

- get introduced to basics in economy, team and project management, ethics and empathy and other 21 st century soft skills;

\section{Results}

This approach was used in our lab in the recent past to start many clinical research projects and produced many papers, patents, and start-ups. We also have started a database of unmet clinical needs that we will make public domain in the coming months.

\section{Conclusion}

Incremental Innovation will continue to be very important for research activities in our field, but we believe that researchers and students should be introduced to disruptive innovation approaches. They should also know basics of management, economics, entrepreneurship, and clinical translation activities to identify and work on ideas that have a significant impact. The methodology that we are using could be a base for more disruption.

\section{References}

[1] Friebe M (2020) Healthcare in need of innovation: exponential technology and biomedical entrepreneurship as solution providers. Proc. SPIE 11315, Medical Imaging 2020: ImageGuided Procedures, Robotic Interventions, and Modeling, 113150T (16 March 2020); https://doi.org/10.1117/12.2556776

[2] Friebe M (2017) Exponential Technologies + Reverse Innovation $=$ Solution for Future Healthcare Issues? What Does It Mean for University Education and Entrepreneurial Opportunities? Open Journal of Business and Management, 5, 458-469. https://doi.org/10.4236/ojbm.2017.53039

\section{$\overline{\text { Technology and global surgery: materials and methods }}$ to achieve the United Nations healthcare-related sustainable development goals for 2030}

\author{
R. Andrews ${ }^{1}$ \\ ${ }^{1}$ NASA Ames Research Center, Nanotechnology \& Smart Systems, \\ Los Gatos, United States
}

Keywords global surgery, mass casualty disaster, resilient \& mobile hospitals, e-health \& telemedicine

\section{Purpose}

In 2015 the United Nations (UN) issued 17 Sustainable Development Goals (SDGs) for 2030, Goal \# 3 being "Ensure healthy lives and promote well-being for all at all ages". Improved global surgery is essential to achieve SDG \#3:

1. One-third of all deaths worldwide result from lack of surgery10 times the deaths due to malaria, tuberculosis, or HIV/AIDS. By 2030, the cost of lack of surgery in low- and middle-income countries (LMICs) - in terms of GDP lost-will approach US $\$ 1.5$ trillion annually. For every dollar invested in global surgery, the long-term savings are greater than 20-fold.

2. Natural disasters annually cost over US $\$ 500$ billion and force over 26 million people into poverty. Annual deaths from earthquakes alone exceed the number killed in traffic accidents in North America and the European Union combined.

3. Man-made or "un-natural" disasters (infrastructure failures, transportation accidents, terrorist/warfare events) kill several hundred thousand people each year worldwide. Deaths and injuries from terrorist events in particular have increased in the past decade.

For daily care, lack of both surgical resources and resilient infrastructure (e.g. power outages) are frequent contributors to avoidable morbidity/mortality. In India alone, deaths due to lack of surgery for acute abdomen number 50,000 yearly; avoidable deaths from trauma, stroke, difficult childbirth, etc., number in the hundreds of thousands annually.

An estimated 20,000 people died each day immediately following the 2010 Haiti earthquake due to lack of basic surgery. Mass casualty disaster (MCD) response in LMICs presently depends on international organizations (e.g. UN, World Health Organization (WHO), Red Cross) and other groups (e.g. military, faith-based). These groupsseparate from the ongoing local healthcare system-require bureaucratic authorizations before mobilization: it is typically a week before medical personnel reach an MCD site. This is far beyond the 12- to 24-hours essential to reduce morbidity/mortality from trauma [1, 2].

Figure 1 illustrates parallels in disaster management and global surgery.

Healthcare delivery resembles smartphones-sophisticated hardware and optimized software as well as a global network are essential for effective operation. Computer-assisted technology can address the need for surgery worldwide. 


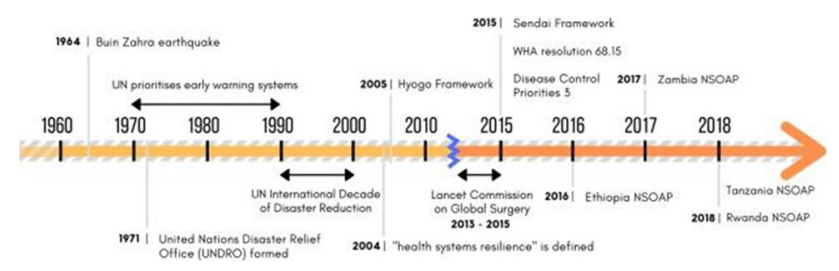

Fig. 1 Timeline of key events in disaster management and global surgery policy since 1960. NSOAP: National Surgical, Obstetric, and Anesthesia Plan; UN: United Nations; WHA: World Health Assembly. BMJ Global Health 2019;4:e001493. https://doi.org/10.1136/ bmjgh-2019-001493

\section{Methods}

Trauma and stroke centers (TSCs) evolved in high-income countries (HICs) with evidence that immediate 24/7/365 treatment dramatically improved morbidity/mortality. TSCs are part of the ongoing healthcare system-not a separate entity. The universal humanitarian response to MCDs suspends political, cultural, and socioeconomic barriers that hinder a coordinated response to other global crises: groups frequently at odds with each other unite during an MCD.

MCD response-like TSCs - can be integrated into ongoing healthcare systems with Mass Casualty Centers (MCCs). Each MCC, like a TSC, is staffed by specialists from all aspects of emergency response-available 24/7/365. Integrating the civilian and military medical resources improves efficiency and minimizes duplication. MCCs in LMICs can be staffed (at least initially) by local physicians and nurses working side-by-side with HIC physicians and nurses (on a rotating basis), following the "twinning" model for partnering medical centers in LMICs and HICs.

Computer-assisted technology has enhanced both daily healthcare and MCD response to both natural and un-natural (man-made) MCDs. A common electronic health record and data collection platform across MCCs enhances standardization of guidelines, training, and quality assurance. Telemedicine can reduce morbidity/mortality and expense in both daily care and MCDs-and also provide immediate 24/7/365 telesurgical guidance. Battery-powered CT scanners provide resilient infrastructure during both power outages (common in LMICs) and MCDs. Mobile operating rooms-portable by helicopter-enable surgery anywhere worldwide within hours. Drones and robots improve both daily healthcare (e.g. transport blood products, lab specimens, vaccines) and MCD response (e.g. identify the living buried in rubble, triage medical resources to maximize benefit).

Resilient, full-service, 24/7/365 MCCs augment the healthcare resources of the region served during non-MCD times (like TSCs in HICs): they provide radiology, blood bank, laboratory, critical care, etc. Groups with resources to optimize cost-effective, immediate care include:

1. The International Virtual eHospital (IVeH) has developed telemedicine programs for Albania, Cabo Verde, the Philippines, and the North Atlantic Treaty Organization (NATO).

2. The Apollo Telemedicine Network Foundation (ATNF), based in India, provides daily telemedicine consultation services to over 30 countries (mostly in sub-Saharan Africa).

3. The Center for Robot-Assisted Search and Rescue (CRASAR) at Texas A\&M University provides immediate free robots and drones for MCD response.

\section{Results}

The initial MCC sites are Iquique (northern Chile) and Peshawar (northwest Pakistan). In Iquique, a joint meeting was held in 2018 with the local health authorities, the military (Chilean Iquique Air Force Base), and the Chilean Ministry for Emergency Response
(ONEMI). In 2019, meetings were held with the Ministry of Health and the Chilean Naval Hospital Director.

In Peshawar, neurosurgeon Tariq Khan over the past decade has opened two hospitals, a medical school (100 students/year), a nursing school (50 students/year), and a ground ambulance service. Community-based trauma prevention and rehabilitation programs have been implemented; the Peshawar Chapter of the ThinkFirst Trauma Injury Prevention Program (begun in the USA in 1986) received the 2019 International Achievement Award. The Military Commander for the Pakistan"s Northern Region supports the MCC project-meetings were held before the COVID-19 pandemic. Meetings with the Surgeon General of Pakistan are planned for 2021, and the MCC Project is coordinating with the Pakistani National Vision for Surgical Care 2025 and NSOAP (National Surgical, Obstetric, and Anesthesia Plan of the WHO) projects.

\section{Conclusion}

Technical advances in resilient and mobile imaging and surgery, electronic data collection and analysis, telemedicine/telesurgery, robots/drones make improvement in both daily healthcare and MCD response not only feasible but economically essential.

MCCs implement universal standards for medical/surgical training, and provide an unmatched global platform for research. They foster camaraderie between physicians and staff from various LMICs and HICs, and development of cost-effective medical/surgical techniques. MCCs advance healthcare and economic progress in LMICs and can be key to realizing many healthcare-related SDGs for 2030 .

There are substantial political and socioeconomic benefits-beyond the healthcare benefits - of integrated MCCs as a means to leverage technology for improved global surgery.

\section{References}

[1] Khan T, Quintana L, Aguilera S, Garcia R, Shoman H, Caddell L, Latifi R, Park KB, Garcia P, Dempsey R, Rosenfeld JV, Scurlock C, Crisp N, Samad L, Smith M, Lippa L, Jooma R, Andrews R (2019). Global health, global surgery, and mass casualties: I. Rationale for integrated mass casualty centres. BMJ Global Health 2019;4:e01943. https://doi.org/10.1136/ bmjgh-2019-001943

[2] Aguilera S, Quintana L, Khan T, Garcia R, Shoman H, Caddell L, Latifi R, Park KB, Garcia P, Dempsey R, Rosenfeld JV, Scurlock C, Crisp N, Samad L, Smith M, Lippa L, Jooma R, Andrews R (2020). Global health, global surgery, and mass casualties: II. Mass casualty centre resources, equipment, and implementation. BMJ Global Health 2020;5:e001945. https://doi.org/10.1136/bmjgh-2019-001945.

\section{Task allocation using wearable devices for managing mixed human-robot teams within the OR wing}

L. Bernhard ${ }^{1}$, D. Wilhelm ${ }^{1}$

${ }^{1}$ Klinikum rechts der Isar, Technische Universität München, Department of Surgery, Research Group MITI, München, Germany

Keywords mobile robots, task allocation, human-robot collaboration, wearables

\section{Purpose}

Mobile robotic systems are a promising technology for addressing many challenges that today's inpatient facilities are facing. This relates to supporting understaffed clinical teams, taking on non-ergonomic or monotonous tasks, and improving overall efficiency of the system. We refer to such mobile robots for the hospital as autonomously self-navigating clinical assistance systems (ASCAS) and are working toward laying the groundwork for integrating these systems into the clinics of tomorrow. Since, for ethical and 
technological reasons, we do not believe in the vision of a fully automated hospital, we see ASCAS systems as a means to support clinicians, not replace them. We center our concepts around what is best for patients and clinicians, while economic factors are only considered secondarily. As a result, and contrary to holistic automation, humans and robots will need to collaborate as teams within the hospital, which introduces the problem of workload balancing between both sides. Though similar problems have been described in the context of other domains, we argue that the application to the hospital deserves special consideration due to unique characteristics regarding highly dynamic workflows and responsibility towards the patient. In this extended abstract, we present a first approach regarding workload balancing by means of task allocation in mixed human-robot teams collaborating within the operating room wing (OR wing).

\section{Methods}

Within the framework of the research project AURORA, we are currently developing a robotic assistant for the OR that is meant to support the circulating nurses and the surgical team as a whole, by executing tasks within the non-sterile area of the operating theater, such as fetching sterilely packaged materials and adjusting medical devices. According to our vision, the human circulators and the AURORA robots are forming a team, from here on referred to as human-robot circulator team (HRCT), that may receive task assignments from several sources, including the sterilely dressed surgical team, non-sterile OR staff, technical assistants or even other robotic systems.

As a foundation for further investigations on how to allocate tasks intelligently within such an HRCT, we analyzed the tasks and workflows that are currently being executed by (human) circulators in the OR. For that, we recorded circulator activity during 15 cholecystectomies and 5 sigma resections conducted at the surgical department of a university hospital.

\section{Results}

We identified several tasks that are reasonable candidates for both robotic or human execution (beyond those provided by our AURORA robot), including cleaning, moving of objects, disposal of waste and phone management. We also found that the priority and frequency of these tasks may vary significantly depending on the patient condition, OR phase and current overall workload. We concluded that, since there is overlap in human and robot capabilities, a context-aware workload balancing mechanism is required to make best use of the available resources in any given situation and also avoid confusion regarding current responsibilities of individual HRCT members. Additionally, we consider avoidance of some of these tasks by implementation of alternative technologies (automated phone management) as a valid option.

Our task allocation concept considers the following scenario: an HRCT, consisting of multiple human team members of different adeptness and multiple ASCAS systems with different capabilities, is responsible for assisting multiple ORs in parallel and therefore needs to distribute its resources in an intelligent way. Especially in situations of high demand or staff shortage, the choice of task allocation may impact patient wellbeing and staff workload significantly. Consequently, we propose that task allocation should consider these aspects during matchmaking (i.e. task assignment to individual HRCT members), instead of assigning tasks randomly or on a first-in-first-out basis.

Let's assume that a given task can, per se, be executed either by a human or a robot. How can intelligently be decided who the task should be assigned to? We argue that patient wellbeing should be the most important influence on this decision. For example, in situations where materials are required urgently to manage an adverse event during surgery, it should be considered whether the task of fetching these materials can be executed faster by a human or robot. However, as long as there is no disadvantage for the patient, we also want to ease the workload of human HRCT members to improve staff satisfaction. Therefore, the execution of, e.g. monotonous or heavy-lifting tasks should be assigned to robotic team members, if currently available. Finally, in cases where patient well-being or staff ergonomics are not significantly affected, we want to optimize efficiency of the overall workflow to benefit economic interests of the hospital.

We envision the HRCT task allocation process as follows: A task manager module receives incoming task assignments and is responsible for creating a schedule, i.e. the execution plan that dictates which task shall be executed at what time by an available resource (Clearly, this entails much more than the decision whether to allocate the tasks to a human or a robot, however, we will exclude these aspects in the context of this abstract). According to the schedule, the tasks are then dispatched to HRCT members. While robotic team members receive task assignments via standard communication interfaces and protocols (e.g. WIFI and JSON), we propose to use wearable devices for human team members. An incoming task assignment will be displayed on the wearable and the circulator can confirm execution begin via touch or voice interaction. Thus, the task manager is now aware of the circulator currently being occupied and considers this information when updating the schedule. However, in case of an unexpected event with high priority, the circulator may receive the request to pause the current task and execute the new, more urgent task first. The circulator can choose to accept or decline this request, depending on whether an interruption of the initial task is possible or not. As soon as the circulator finishes a task, the successful execution must be reported back to the task manager. Again, this can be achieved using the wearable device.

\section{Conclusion}

In this extended abstract, we introduced the problem of HRCT task allocation and discussed in which order patient well-being, staff ergonomics and the hospital"s economic interests should be an influence on this. Furthermore, we proposed an early concept how we believe wearable devices can be used for allocating tasks to human HRCT members. In the future, we aim at integrating these results into our ANTS-OR scheduling framework to offer support for mixed human-robot teams.

\section{The hospital of the future}

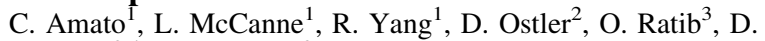
Wilhelm $^{2,4}$, L. Bernhard ${ }^{2}$

${ }^{1}$ CannonDesign, Los Angeles, California, USA ${ }^{2}$ Research Group MITI, Klinikum rechts der Isar, Technical University Munich, Germany ${ }^{3}$ Department of Radiology and Medical Informatics, University of Geneva, Switzerland ${ }^{4}$ Department of Surgery, Klinikum rechts der Isar, Technical University Munich, Germany

\section{Purpose}

Current healthcare systems around the world are characterized by the organizational principles of segmentation and separation of medical specialties. They are structured into departments and facilities which are not tightly linked to the rest of the healthcare ecosystem. With our new hospital concept, called Patient Hub, we envision a one-stop truly patient-centric, department-less facility, where all critical functions occur on one floor and which could serve not only as a model for the hospital, but also for the general healthcare system of the future.

\section{Methods}

The effectiveness and added value of our proposed hospital architecture was benchmarked against a traditional design using a 3D simulation software and by assessing workflow efficiency and patient satisfaction for an exemplary scenario around a patient being diagnosed and treated for rectal cancer. 


\section{Workflow simulation}

Using the 3D simulation software FlexSim Healthcare ${ }^{\mathrm{TM}}$ (FlexSim Software Products, Inc., Orem, Utah, USA) we developed dynamic models for comparing various quality measures between our two different settings. We focused on measuring:

- The time spent on each step of patient clinical pathways

- Estimated bottlenecks and waiting times

- Distance and number of patient transfers

- Required staff size

- Number of waiting rooms and other spaces

\section{Results}

\section{The Patient Hub Concept}

Today's hospital buildings are often versions of the 1960s bed-toweron-diagnostic-and-treatment podium model similar to a Kaiser Permenente hospital, which are separated in several departments and in which the patient is incrementally moved around from place to place to receive care instead of bringing the care to the patient.

Our Patient Hub concept is a radical departure from this design and envisions a transformative "one of a kind", truly patient-centric, department-less facility. We propose a highly centralized clinical layout, where all relevant medical fields of expertise are available within the same space surrounding the patient (see Figure 1). They form clusters which contain functionalities of equal classes, for example inpatient or outpatient care. The centralized clinical layout brings together staff from all specialties to encourage clinical collaboration and care coordination. The Patient Hub co-locates outpatient, inpatient, rehabilitation, wellness and prevention, ancillary support spaces, and R\&D all under one roof. It is no longer a site for the treatment of the sick rather than a health-oriented all-encompassing facility.

By avoiding copies of functionalities in every department (e.g. waiting rooms) the Patient-hub concept allows for the saving of space, for complexity reduction of patient pathways, and for easy implementation of new treatment concepts. While keeping this functional patient-hub design with all required functionalities being logically distributed on one floor, different levels of the building can be adapted to different patient needs and treatment forms (Figure 2). Comparison to traditional hospital layout

Numeric results of the actual workflow simulations are given in Table 1, whereas a more detailed description of the simulation and results will be presented during the congress.

\section{Discussion}

Our (preliminary) results on a simple patient scenario are promising, since a considerable improvement for every selected parameter can be observed. In more complicated situations and workflows we believe the benefits of the new patient-centric layout will become even more

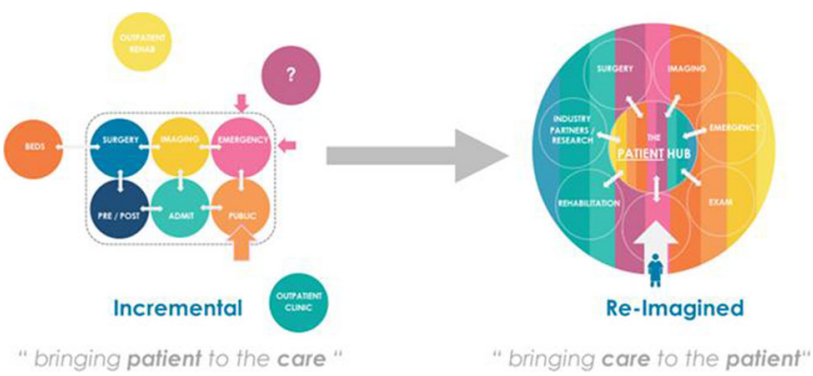

Fig. 1 Re-imagined healthcare paradigm: changing from incremental "patient to care" towards a re-imagined "care to patient" approach

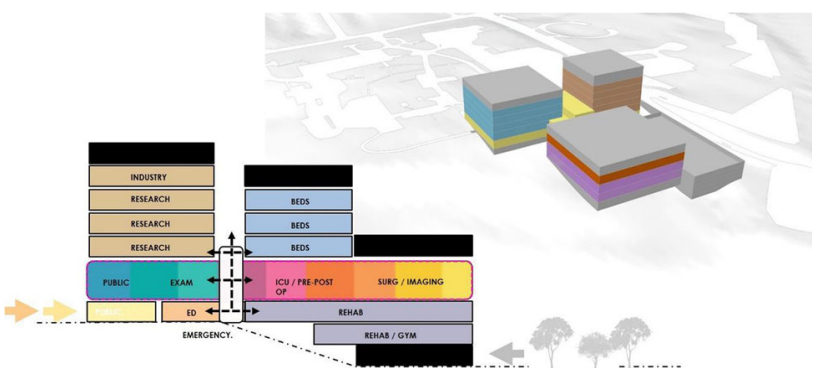

Fig. 1 Patient Hub functional stacking and 3D massing

Table 1 Results of workflow simulation comparison

\begin{tabular}{ll}
\hline Parameter & Result \\
\hline $\begin{array}{l}\text { Number of "stops" or patient "hand-offs" } \\
\text { between staff and departments—-(traveling } \\
\text { from different specialty areas to and from }\end{array}$ & $55 \%$ reduction \\
$\quad$ Medical/Surgical Ward/ICU) & \\
Average elevator travel waiting time & $85 \%$ reduction \\
Elevator Trips & $80 \%$ reduction \\
Reception personnel and waiting rooms needed & $50 \%$ reduction \\
$\begin{array}{l}\text { Trips to administration for interview, receive } \\
\text { data and instructions }\end{array}$ & $75 \%$ reduction \\
$\begin{array}{l}\text { Patient transfer travel distance horizontal } \\
\text { Staff support/commons spaces typically found } \\
\text { and repeated in different departments } \\
\text { (lounges, conference rooms, offices, etcetera) }\end{array}$ & $40 \%$ space savings \\
Universal, one stop patient room (Multi-acuity) & $35 \%$ increase in \\
& room size \\
\hline
\end{tabular}

obvious-due to the reduction of bottlenecks and resulting improvements of target parameters relevant for patient experience.

As of now, the proposed concepts mainly focus on architectural design and a translation to the real world will certainly require many more building blocks, such as AI, big data and robotics. In particular, we envision the entire infrastructure, including technical devices, spaces and functional units to become adaptive, mobile and intelligent. While we plan to incorporate such considerations into future work, we advocate a very deliberate use of technology, goverened by the paradigm of bringing care to the patient and to increase patient satisfaction. Lastly, our simulation results show significant increases in efficiency throughout the facility, with less required staff members and less time required per patient.

\section{Conclusion}

With the Patient Hub concept we envisioned breaking the traditional departmental organization and topological distribution in isolated clusters of today's hospitals and improving patient experience and satisfaction while optimizing the efficiency of therapeutic and diagnostic procedures. We further introduced a more structured approach to design and experiment with the disruptive and innovative architecture of future healthcare facilities. 


\section{5th Annual Conference of the International Society for Computer Aided Surgery}

Chairs: Kensaku Mori, PhD (JP), Cristian A. Linte, PhD (US) 


\section{Combining augmented reality and 3D printing to improve orthopedic oncological surgeries}

\author{
A. Pose Díez de la Lastra ${ }^{1,2}$, R. Moreta Martínez ${ }^{1,2}$, J. A. Calvo \\ Haro $^{2,3,4}$, L. Mediavilla Santos ${ }^{2,3,4}$, R. Pérez Mañanes ${ }^{2,3,4}$, J. \\ Pascau $^{1,2}$
}

${ }^{1}$ Universidad Carlos III de Madrid, Departamento de Bioingeniería e Ingeniería Aeroespacial, Leganés, Spain ${ }^{2}$ Instituto de Investigación Sanitaria Gregorio Marañón, Madrid, Spain ${ }^{3}$ Hospital General Universitario Gregorio Marañón, Servicio de Cirugía Ortopédica y Traumatología, Madrid, Spain ${ }^{4}$ Universidad Complutense de Madrid, Departamento de Cirugía, Facultad de Medicina, Madrid, Spain

Keywords Augmented reality, 3D printing, Orthopedic oncology, Microsoft Hololens

\section{Purpose}

Orthopedic oncology treats tumors affecting bones and soft tissues. In many cases, it involves their complete surgical resection, ensuring a safety margin of healthy tissue. However, these surgeries are a real challenge to clinicians, since local recurrence rate is $27 \%$ and the fiveyear survival rate, $50 \%$ [1].

During the last decade, surgical navigation improved tumor resection accuracy, decreasing local occurrence, and enhancing surgical outcomes. Nevertheless, traditional navigation systems do not always adapt to this kind of interventions. They display real-time navigation information on external screens, requiring the surgeon to move his attention away from the patient.

Three-dimensional printing (3DP) and augmented reality (AR) have recently increased their adoption in many medical areas with exciting benefits. 3DP allows creating patient-specific anatomical biomodels for surgical planning and patient communication. AR enables the simultaneous interaction with real and virtually projected $3 \mathrm{D}$ elements during medical training or surgery. These two technologies could overcome the limitations identified for surgical navigation by displaying relevant patient information on-site during surgical procedures.

In this study, we propose a system that combines both technologies to improve orthopedic oncological surgeries. This solution was developed as an AR-based application for Microsoft HoloLens 2. It displays the internal anatomical structures overlaid on the patient using two 3D-printed tools: a reference marker and a surgical guide. They are designed to fit in a unique position of the bone that surrounds the tumor, thus enabling automatic registration. We assessed this solution on a patient with an undifferentiated pleomorphic sarcoma on the right deltoid, measuring the precision with a 3D-printed phantom obtained from the patient data and evaluating the performance during the actual surgical intervention.

\section{Methods}

An AR-based HoloLens application was developed on Unity platform. The app uses the camera and Vuforia ${ }^{\circledR}$ development kit to identify a two-dimensional 3D-printed reference marker that contains a known black and white pattern. Once the marker is identified, virtual models are projected over the patient to guide the surgeon during the tumor identification facilitating the resection. Three virtual models (tumor, surrounding bone, and surgical guide) can be displayed with user-selected transparency.

The bone and the tumor were segmented from the CT of the patient using 3D Slicer platform. The surgical guide was created using MeshMixer software as a negative of the bone's surface, with a support to fix the AR marker. The surgical guide was printed in BioMed Clear V1 resin material (class II biocompatible material) on a Form 2 stereolithography 3D printer. Both the guide and the AR marker were sterilized before surgery with ethylene oxide $(\mathrm{EtO})$ at $55^{\circ} \mathrm{C}$ and $37^{\circ} \mathrm{C}$, respectively. The relative coordinates of the biomodels and the marker were computed and stored using a previously developed 3D Slicer module. Once the marker is detected, the virtual models are automatically displayed in the correct position with respect to the patient.

Prior to the surgery, the precision of the system was evaluated at the laboratory using patient-specific phantom, 3D-printed in PLA with an Ultimaker 3 Extended 3D printer, containing the tumor and the surrounding bone. We also 3D printed a version of the surgical guide in resin. Both phantom and guide included eight and four conical holes respectively ( $\varnothing 4 \mathrm{~mm} \times 3 \mathrm{~mm}$ depth) as reference landmarks for the experiment. We used a Polaris Spectra optical tracking system and a pointer to record the reference fiducials' positions, allowing to calculate the registration (phantom'landmarks) and two precision values: surgical guide placement error (guide'landmarks) and the AR tracking error. We virtually augmented 14 randomly distributed spheres $(\varnothing 3 \mathrm{~mm})$ on the phantom's surface to measure the AR error. Two users selected with the pointer the position of each conical hole or sphere (guided by the AR app). The error corresponded to the distance between the pointer position and the known sphere coordinates. Each user repeated the process five times.

\section{Results}

The errors obtained on the phantom are summarized in Table 1. The mean surgical guide placement error was $1.34 \pm 0.39 \mathrm{~mm}$, and the overall AR error $2.38 \pm 0.90 \mathrm{~mm}$. Both values are slightly better than recent literature in AR surgical guidance with Hololens 1 [2].

In the surgical room, the surgical guides fitted as planned in the target area on the patient's bone, and the AR marker could be correctly detected by Hololens 2 . Hence, we could calculate a successful registration between the AR system and the patient's anatomy, projecting the virtual biomodels overlayed on the patient in their expected location (see Figure 1). Surgeons' feedback on the intuitiveness and comfortability of the system was very positive. They believe that this AR solution could increase the accuracy of the procedure and boost their confidence to verify that the tumor has been correctly resected.

\section{Conclusion}

The results obtained in both scenarios demonstrate the benefits that the combination of AR and 3DP can bring to orthopedic oncological surgeries. The laboratory results show precision values in concordance with current literature, and surgeons' feedback endorses the applicability of our proposal from the clinical perspective, promoting further research on this area. More cases with different tumor conditions will be studied to warranty the usability of the system. This work establishes a baseline for developing more AR and 3DP systems for surgical guidance in orthopedic oncology in the future.

\section{Acknowledgements}

I would like to thank my parents for their endless support, thanks to my companions for their guidance and advice, and thanks to Luis for everything.

Table 1 Mean surgical guide placement error and augmented reality tracking error per user

\begin{tabular}{lccc}
\hline & User 1 & User 2 & Overall \\
\hline \multicolumn{2}{l}{ Surgical guide placement error (mm) } & & \\
Mean & 1.34 & 1.33 & 1.34 \\
SD & 0.31 & 0.46 & 0.39 \\
Augmented reality & tracking error (mm) & \\
Mean & 2.43 & 2.31 & 2.38 \\
SD & 0.86 & 0.95 & 0.90 \\
\hline
\end{tabular}




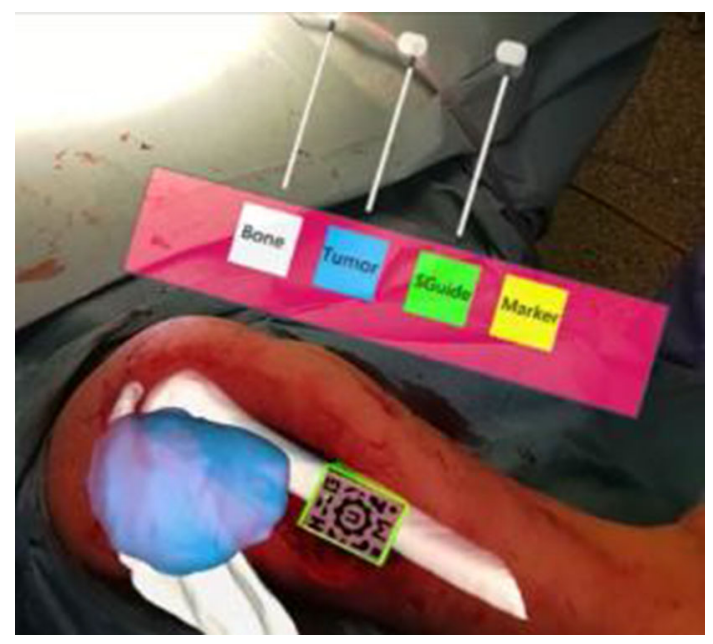

Fig. 1 HoloLens view during the surgery after the tumor resection. The virtual bone and tumor are projected overlaid on the patient in the correct position. A virtual panel allows controlling the transparency of the virtual biomodels

\section{References}

[1] Clark MA, Fisher C, Judson I, Thomas JM (2005) Soft-Tissue Sarcomas in Adults. N Engl J Med 353:701-711. https://doi.org/10.1056/NEJMra041866

[2] Moreta-Martinez R, García-Mato D, García-Sevilla M, PérezMañanes R, Calvo-Haro J, Pascau J (2018) Augmented reality in computer-assisted interventions based on patient-specific 3D printed reference. Health Technol Lett 5:162-166. https://doi.org/10.1049/htl.2018.5072

\section{Development of an electromagnetic catheter tracking system using TMR sensor for superselective intra- arterial chemotherapy}

R. Nagano ${ }^{1}$, K. Hara $^{2}$, A. Ishijima ${ }^{1}$, N. Tomii ${ }^{1}$, K. Nakagawa ${ }^{3}$, Y. Akagi $^{3}$, M. Yamazaki ${ }^{3}$, H. Tsukihara ${ }^{2}$, E. Kobayashi ${ }^{1}$, T. Ohya ${ }^{4}$, I. Sakuma ${ }^{1,2,3}$

${ }^{1}$ The University of Tokyo, Graduate School of Engineering, Department of Precision Engineering, Tokyo, Japan ${ }^{2}$ The University of Tokyo, Medical Device Development and Regulation Research Center, Tokyo, Japan ${ }^{3}$ The University of Tokyo, Graduate School of Engineering, Department of Bioengineering, Tokyo, Japan ${ }^{4}$ Yokohama City University, Graduate School of Medicine, Department of Oral and Maxillofacial Surgery, Yokohama, Japan

Keywords Tunneling magnetoresistance, Electromagnetic tracking, Catheter navigation, Oral cancer

\section{Purpose}

Superselective intra-arterial chemotherapy is an effective treatment for oral cancer in that surgeons can provide a high concentration of anticancer drugs into the tumor-feeding arteries through a catheter [1]. In general, X-ray fluoroscopy is used to track the position and orientation of the catheter"s tip for inserting it into the targeted artery. However, it requires X-ray exposure and administration of contrast agents. Electromagnetic tracking is often used to track medical instruments inside a human body [2]. Although electromagnetic wave is less invasive than $\mathrm{X}$-ray, the size of conventional coil sensors is too large $(>5.5 \mathrm{~mm})$ to fit inside the catheter $(<1.3 \mathrm{~mm})$ for superselective intra-arterial chemotherapy. For this purpose, tunneling magneto-resistance (TMR) sensor $(\sim 0.3 \mathrm{~mm})$ that measures magnetic fields based on the tunneling magnetoresistance effect is a promising candidate to use as a magnetic sensor in the electromagnetic system. However, its nonlinear field-voltage characteristics needs to be considered to implement TMR sensors in an electromagnetic tracking system. Here, we proposed a TMR sensor based electromagnetic tracking system considering its nonlinear field-voltage characteristics and evaluated the position estimation accuracy of the system.

\section{Methods}

The TMR sensor based electromagnetic tracking system consists of a field generator and a TMR sensor device. For the field generator, six inductors were driven simultaneously with different frequencies. The magnetic field strength of the six inductors was limited to \pm 398 $\mathrm{A} / \mathrm{m}$, so as not to exceed the range in which the field-voltage characteristics of the TMR sensor were bijective. For the TMR sensor device, we used a custom-made three axis TMR sensor (ABA03, Daido Steel, JP). The output voltages were converted to magnetic field values by a quadratic function fitted nonlinear fields-voltage characteristics. The converted magnetic field values were decomposed into each frequency using phase detection. Calibration was performed to compensate fields distortion before position calculation. Magnetic fields distortions are caused by eddy currents in surrounding conductive materials and mutual induction between inductors. The magnetic fields" values were compensated by a predetermined calibration matrix. The calibration matrix was determined by measuring the magnetic fields at 45 known positions and minimizing the difference between the measured values and theoretical values with the least-squares method.

The position estimation accuracy of the system was evaluated by the experimental setup shown in Fig. 1. The inductors used in the experiment were $100 \mathrm{~mm}$ in diameter with 6060 turns. The inductor frequencies were set to $355,435,602,748,881,1061 \mathrm{~Hz}$. The sensor device was located at 45 points using positioning pins within $100 \mathrm{~mm} \times 200 \mathrm{~mm} \times 100 \mathrm{~mm}$. The position of the sensor device was calculated by using the Levenberg-Marquardt method which searches for a point where the compensated measurement value matches the theoretical value. The estimated position was iteratively updated until the position difference became less than $0.1 \mathrm{~mm}$. The errors between the estimated sensor position and the positioning pins were evaluated $(N=4)$.

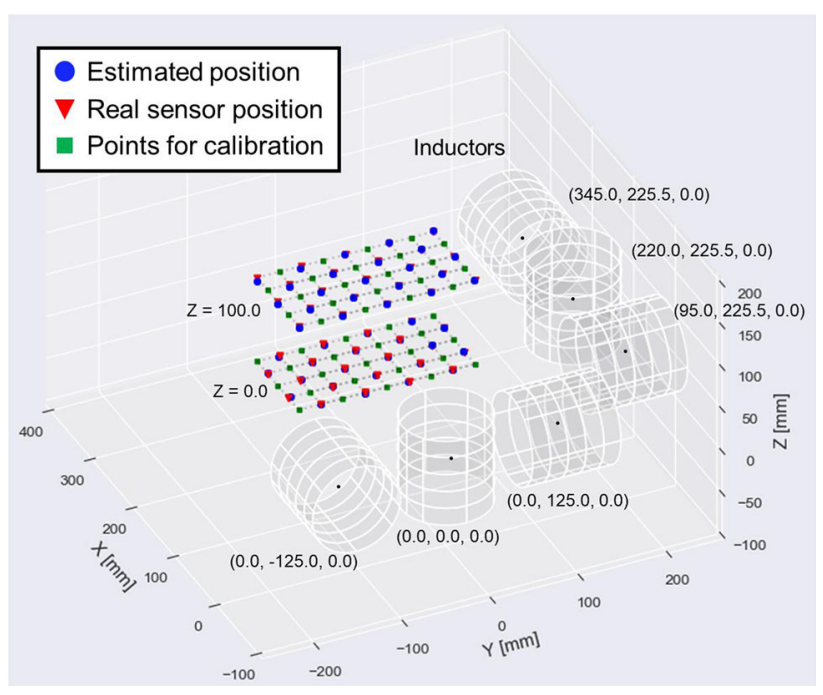

Fig. 1 Position estimation of a TMR sensor with developed tracking system 
Table 1 Errors and standard deviations (STD) of 45 points

\begin{tabular}{ccccccc}
\hline & & & Mean & Median & Min. & Max. \\
\hline $\begin{array}{c}\text { Error } \\
(\mathrm{mm})\end{array}$ & $\begin{array}{c}\text { Euclidean } \\
\text { norm }\end{array}$ & All (45points) & 1.24 & 0.88 & 0.18 & 4.62 \\
& & $\mathrm{Z}=0(22$ & 0.85 & 0.53 & 0.18 & 3.54 \\
& & points) & & & & \\
& & $\mathrm{Z}=100(23$ & 1.53 & 1.19 & 0.29 & 4.62 \\
& & points $)$ \\
& X-axis & All (45points) & 0.74 & 0.45 & 0.08 & 3.71 \\
& Y-axis & All (45points) & 0.55 & 0.33 & 0.06 & 3.06 \\
& Z-axis & All (45points) & 0.55 & 0.26 & 0.04 & 3.60 \\
SD & Euclidean & All (45points) & 0.49 & 0.41 & 0.10 & 2.21 \\
& norm & Z = 0 (22 & 0.33 & 0.29 & 0.10 & 0.72 \\
& & points) & & & \\
& & Z = 100 (23 & 0.70 & 0.54 & 0.16 & 2.21 \\
& & points) & & & \\
& X-axis & All (45points) & 0.38 & 0.24 & 0.05 & 2.62 \\
& Y-axis & All (45points) & 0.27 & 0.21 & 0.05 & 0.96 \\
& Z-axis & All (45points) & 0.29 & 0.26 & 0.02 & 0.80 \\
\hline
\end{tabular}

\section{Results}

The results are shown in Table 1 and Fig. 1. The mean error of 45 points was $1.24 \mathrm{~mm}$. The smallest error was $0.18 \mathrm{~mm}$ at $(242.5,75.0$, $0.0)$, while the largest error was $4.62 \mathrm{~mm}$ at $(242.5,-100,100)$. The errors tended to increase as the distance from the inductors increased. The mean error of 22 points on the $Z=0.0 \mathrm{~mm}$ plane was $0.85 \mathrm{~mm}$, while the mean error of 23 points on the $Z=100.0 \mathrm{~mm}$ plane was $1.53 \mathrm{~mm}$. For each axis, the mean error was $0.74 \mathrm{~mm}$ for the $\mathrm{x}$-axis, $0.55 \mathrm{~mm}$ for the $y$-axis, $0.55 \mathrm{~mm}$ for the $\mathrm{z}$-axis.

The mean standard deviation of 45 points was $0.49 \mathrm{~mm}$. The smallest standard deviation was $0.10 \mathrm{~mm}$ at $(142.5,75.0,0.0)$, while the largest standard deviation was $2.11 \mathrm{~mm}$ at $(242.5,-50.0,100.0)$. The standard deviations also tended to increase as the distance from the inductors increased. The mean standard deviation of 22 points on the $\mathrm{Z}=0.0 \mathrm{~mm}$ plane was $0.33 \mathrm{~mm}$, while the mean standard deviation of 23 points on the $Z=100.0 \mathrm{~mm}$ plane was $0.70 \mathrm{~mm}$. For each axis, the mean standard deviation was $0.38 \mathrm{~mm}$ for the $\mathrm{x}$-axis, $0.27 \mathrm{~mm}$ for the y-axis, $0.29 \mathrm{~mm}$ for the $\mathrm{z}$-axis.

\section{Conclusion}

We have constructed and evaluated the position estimation accuracy of an electromagnetic tracking system using a TMR sensor. The mean error of sensor position estimation was $1.24 \mathrm{~mm}$. Although the accuracy of sensor orientation has not been evaluated, TMR sensor is a promising candidate to use as a magnetic sensor for an electromagnetic tracking system in superselective intra-arterial chemotherapy. (Funding availability: Grant-in-Aid for Scientific Research (B) (JSPS KAKENHI Grant Number JP 20H04553), JSPS, Japan)

\section{References}

[1] Tohnai I, Fuwa N, Hayashi Y, Kaneko R, Tomaru Y, Hibino Y, Ueda M (1998) New superselective intra-arterial infusion via superficial temporal artery for cancer of the tongue and tumour tissue platinum concentration after carboplatin (CBDCA) infusion. Oral oncology 34(5):387-390

[2] Andria G, Attivissimo F, Di Nisio A, Lanzolla AML, Larizza P, Selicato S (2019) Development and performance evaluation of an electromagnetic tracking system for surgery navigation. Measurement 148:106916.

\section{Developing an MRI-compatible concentric tube robot for intracerebral hemorrhage evacuation in the bore of the magnet}

Y. Chen ${ }^{1}$, I. Godage ${ }^{2}$, S. Sengupta ${ }^{3}$, D. Sigounas ${ }^{4}$, C. Kellner ${ }^{5}$, K.

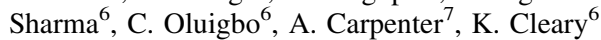

${ }^{1}$ University of Arkansas, Fayetteville, United States ${ }^{2}$ DePaul

University, Chicago, United States ${ }^{3}$ Vanderbilt University, Nashville, United States ${ }^{4}$ George Washington University, Washington, United

States ${ }^{5}$ Mount Sinai, New York, United States ${ }^{6}$ Children's National Hospital, Washington, United States ${ }^{7}$ Georgetown University, Washington, United States

Keywords robotics, interventions, MRI, hemorrhage

\section{Purpose}

The goal of this project is to develop and evaluate a minimally invasive MRI compatible concentric tube robot for intracerebral hemorrhage (ICH) evacuation under interventional MRI (iMRI). While the current standard for ICH evacuation uses CT imaging, we aim to explore whether ICH evacuation under iMRI is feasible and could provide a new treatment paradigm for these procedures. The advantages of MRI versus CT for the brain include the delineation of white matter tracts in optimizing a surgical corridor to the hemorrhage; greatly enhanced visualization of underlying pathology, critical structures, and hemorrhage heterogeneity impacting the degree of evacuation; and lack of radiation which means multiple scans can be acquired during the procedure.

About 1 in 50 people suffer from ICH in their lifetime and is the leading cause of long-term disability in the United States. ICH occurs when blood leaked from a ruptured vessel accumulates and forms a blood clot (hematoma) in the brain parenchyma, which can compress local structures. The 30 -day mortality for ICH is about $40 \%$ with half of all deaths occurring in the acute phase, especially in the first $48 \mathrm{~h}$. Blood spilled outside of the intracranial vessels is toxic to the surrounding brain, causing inflammation and secondary brain injury. This manifests as edema seen immediately after the hemorrhage and increases rapidly over the first $24 \mathrm{~h}$. The aim of surgical intervention is decompression to relieve the mass effect exerted by the hematoma on the brain. Traditional surgical techniques do not always offer clinical benefit and often disrupt more normal brain in the operative approach than the volume of brain that could be saved by evacuating the clot. These deleterious effects motivate improved treatments to save at-risk brain tissue. Therefore, a minimally invasive approach under MRI guidance may offer increased accuracy and the ability to decompress the brain with less disruption to normal brain in approaching the hematoma.

Methods

Our system concept is shown in the figure 1 which includes (1) an MRI-compatible concentric tube robotic system positioned on a 6-DoF supporting arm; (2) a navigation workstation to visualize the cannula and brain/hematoma MRI images; and (3) a user input device for the clinician to control the concentric tube robot to evacuate the clot.

The robot will be fabricated using 3D printing techniques and incorporate novel MRI-safe actuators capable of accurate movement in the bore of the magnet. Actively tracked microcoils mounted on the aspiration cannula will enable sub-millimeter position feedback for accurate cannula localization. Real-time image feedback from MRI will enable intraoperative monitoring for deploying the aspiration 


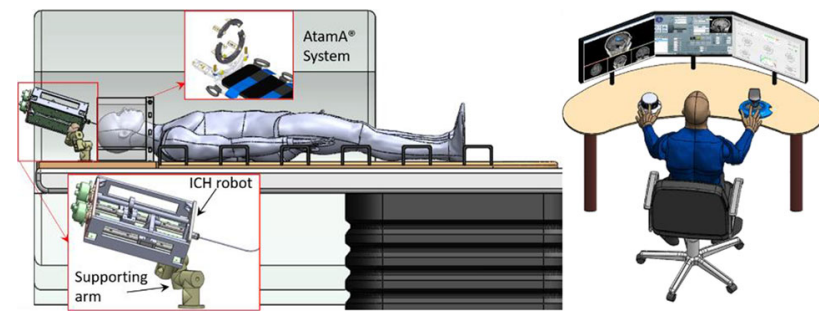

Fig. 1 (Left) Schematic diagram of the 3D-printed robot inside the MRI scanner. The top inset shows the AtamA patient transfer and head stabilization system. The left bottom inset shows the robot module mounted on a passive supporting arm. (Right) The surgeon sits inside the MRI control room and uses a joystick (right hand) to manipulate the aspiration cannula and a proportional vacuum regulator (left hand) to control the aspiration under MRI guidance

cannula deployment within the hematoma. MRI will also help monitor the treatment outcome to avoid incomplete or overly aggressive hemorrhage evacuation.

\section{Results}

We have created a prototype MRI-compatible concentric tube robot for ICH aspiration. It consists of an MR-safe actuation unit that applies the translational and rotational motions to the concentric tubes. The robot is mounted on top of a 6-DOF stereotactic supporting arm to enable the correct orientation towards the hematoma. We have tested the concentric tube robot workspace in ICH models, segmented from brain images from 20 patients provided by Vanderbilt University Medical School under an approved IRB protocol, and found the aspiration cannula could reach and cover all the hematomas. A benchtop targeting accuracy characterization study was performed using an electromagnetic tracker (Aurora, Northern Digital, Inc.). The experimental results indicated the prototype has a targeting accuracy of $1.26 \pm 1.22 \mathrm{~mm}$ [1]. The MRI-guided phantom study showed that the robot successfully reached the desired target with real-time MRI guidance and evacuated approximately $11.3 \mathrm{ml}$ of phantom hematoma in $9 \mathrm{~min}$. The complete setup was tested in a 3T MR scanner and no image distortions or safety hazards were observed based on ASTM standards tests.

\section{Conclusion}

We will build on the prototype system described above to expand its capabilities including real-time MRI guided concentric tube robot control and efficient hemorrhage evacuation with intraoperative monitoring. Future work will also include cadaver tests and swine animal studies.

\section{References}

[1] Chen Y, Godage IS, Sengupta S, Liu CL, Weaver KD, Barth EJ (2019) MR-conditional steerable needle robot for intracerebral hemorrhage removal. International journal of computer assisted radiology and surgery, 14(1), pp. 105-115.

\section{Real-time automated performance metrics for mastoidectomy surgical simulation}

\author{
E. Simpson ${ }^{1}$, S. Agrawal ${ }^{1,2,3,4}$, H. Ladak ${ }^{1,2,3,4}$ \\ ${ }^{1}$ Western University, Electrical and Computer Engineering, London, \\ Canada ${ }^{2}$ Western University, Department of Otolaryngology-Head \\ and Neck Surgery, London, Canada ${ }^{3}$ Western University, Department \\ of Medical Biophysics, London, Canada ${ }^{4}$ Western University, School \\ of Biomedical Engineering, London, Canada
}

Keywords Metrics, Mastoidectomy, Simulation, Bone drilling

\section{Purpose}

Mastoidectomy (temporal bone drilling) is difficult to master and requires significant training. Cadaveric practice material is expensive and difficult to obtain, resulting in the closure of many temporal bone courses in North America. Additionally, an expert surgeon must be present to evaluate the drilled cadaveric sample. These requirements hinder the accessibility of surgical training in otology. An adjunct to cadaveric practice is a virtual reality simulator designed for temporal bone drilling. Western University, University of Calgary, and Stanford University have developed a virtual surgical environment called CardinalSim that allows the user to view and drill patient-specific digital models of anatomical structures as well as receive intuitive haptic (touch) feedback from simulated tools. Whilst CardinalSim removes the need for cadaveric material and provides summative feedback automatically, it does not yet provide formative feedback and an expert is required to monitor the training session. Hence, the purpose of this work is to implement real-time metrics to provide formative feedback.

\section{Methods}

Software technologies including voxel representation, ray-casting, and asynchronous scheduling were employed to create an automated real-time metric system. Algorithms have been developed to dynamically monitor and evaluate CardinalSim"s virtual surgical environment, haptic tool, and digital anatomical structures in realtime. User drilling interaction is sequenced into a series of drilling strokes that contain information regarding the applied pressure, bone mass removed, and time required for removal. These data points are recorded to volatile memory and analyzed for indication of poor surgical practice. After the user completes a drill stroke (signified by de-actuating the haptic tool), the stroke is further analyzed to calculate metrics and statistics pertaining to the overall surgical procedure progress.

This version of CardinalSim allows the user to initiate an evaluated procedure by importing and signifying critical structures and the evaluation metrics to process. Calculated metric data are presented to the user as raw values, visual queues, and textual feedback in the form of numerical fields, colour indicators, and a progress-feed. These user-interface elements can be displayed as a side-panel next to the surgical view or as a secondary view on an additional display. In order to maintain user-interface reliability and performance, an asynchronous system was developed that ensures every data point is recorded and the most recently analyzed data are displayed to the user. Finally, when concluding the virtual procedure, a post-operative report is displayed.

\section{Results}

Real-time automated metrics have been developed to identify poor practices such as sporadic or hesitant drilling, drilling whilst the drill burr is not visible, drilling forcibly with the bottom-side of the burr, and using a burr that is either too large or too coarse (see Figure 1). Overall procedure progress has also been quantified to provide the total amount of volume removed, length of time spent drilling, length of time taken to drill out critical landmark structures, average force applied, and average distance between removed bone material.

Metrics that indicate poor practice have been evaluated with various acceptance tests wherein a user with adept surgical knowledge processes the corresponding poor and proper actions to verify that the system can detect and record them effectively. This was furthered by thorough alpha and ad-hoc testing in virtual user environments and workflows during the agile development phase. Numerical-based metrics such as those involving volume were verified by processing pre-programmed surgical scenes generated outside of CardinalSim to ensure known values were obtained. Numericalbased metrics involving time and force were tested by comparing the results to external monitoring applications such as those included with the haptic arm. 


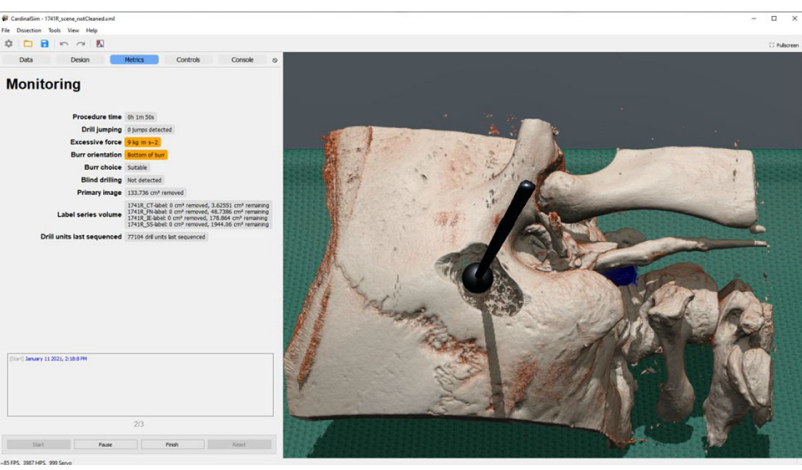

Fig. 1 CardinalSim surgical view with metrics

The asynchronous design resulted in no noticeable performance impact on the simulator when compared to the same system with metrics disabled. During testing, frames per second was reduced by an average of $3 \%$ while metric calculation was occurring. Thus, user metric feedback was able to formulate and display as the user interacts with the system in real-time.

Clinical residents are currently being recruited to participate in a validation study to determine efficacy of the metrics in discriminating surgical performance.

\section{Conclusion}

Real-time automated metrics have been developed to identify poor practice (sporadic or hesitant drilling, drilling whilst the drill burr is not visible, drilling forcibly with the bottom-side of the burr, and using a burr that is either too large or too coarse) and highlight overall procedure progress (total amount of volume removed, the length of time spent drilling, the length of time taken to drill out critical landmark structures, average force applied, and average distance between removed bone material). The user receives formative feedback as raw values, visual queues, and text in the form of numerical fields, colour indicators, and a progress-feed. Real-time performance was maintained with the use of an asynchronous sequencing and analysis design that dynamically monitors user interaction. The addition of a real-time automated metric feedback system will allow users to learn mastoidectomy when an expert surgeon is not present, thus saving valuable human resources.

\section{D cryo-balloon catheter orientation reconstruction based on automatically detected structures in 2D \\ X-ray fluoroscopy}

D. Bertsche ${ }^{1}$, T. Dahme ${ }^{1}$, W. Rottbauer ${ }^{1}$, V. Rasche ${ }^{1}$, I.

Vernikouskaya $^{1}$

${ }^{1}$ Ulm University Medical Center, Clinic of Internal Medicine II, Ulm, Germany

Keywords cryo-balloon ablation, pulmonary vein isolation, 3D reconstruction, image fusion

\section{Purpose}

Cryoablation is a popular treatment for atrial fibrillation. The mainly $\mathrm{X}$-ray fluoroscopy (XR)-guided procedure is performed by placing the cryo-balloon $(\mathrm{CB})$ in the pulmonary vein ostia and filling the $\mathrm{CB}$ with refrigerant to electrically isolate the veins from the atrium by annular scarring. High variability of pulmonary veins (PV) and acute angulation of $\mathrm{PV}$ challenges proper $\mathrm{CB}$ positioning [1]. However, to achieve sufficient $\mathrm{PV}$ isolation, the $\mathrm{CB}$ must be in optimal position in relation to the PV. Insufficient isolation may require re-intervention. To facilitate correct $\mathrm{CB}$ positioning, visualization of the $\mathrm{CB}$ location in relation to the target structure might be helpful. In this work, we propose to reconstruct the $3 \mathrm{D} \mathrm{CB}$ orientation from automatically detected radio-opaque marker and catheter shaft in biplane XR fluoroscopy to visualize the $\mathrm{CB}$ position relative to the patient specific left atrium. Based on the position of the radio-opaque marker and the course of the catheter shaft, detected in the biplane XR fluoroscopy using two neural networks, the $\mathrm{CB}$ orientation was reconstructed in $3 \mathrm{D}$ and fused with the patient specific 3D model of the left atrium and its centerlines. The $\mathrm{CB}$ orientation in relation to the target structures could achieve improved navigation during the procedure or may enable accurate pre-procedural planning for re-intervention.

\section{Methods}

The radio-opaque XR marker and the cryo-balloon catheter shaft were automatically segmented in corresponding biplane fluoroscopic images using neural networks of U-net architecture [2]. The segmented marker and catheter shaft masks were post-processed to obtain: (1) a single seed point as the center of the detected marker contour and (2) the spline fitted catheter centerline from analytical graph of the skeletonized networks output. Based on the automatically detected structures in biplane images the 3D orientation of the $\mathrm{CB}$ was reconstructed. The marker position was reconstructed based on epipolar geometry from (1). From (2), a direction vector was fitted using minimal averaged Euclidean distance measurement (Fig. 1a). According to the projection geometries, the direction vectors were translated in order to obtain initialization by the previously reconstructed marker position. Using the cross product, the normal to the plane spanned by the projection vector and direction vector was calculated for both of the $\mathrm{C}$-arm configurations. The cross product of the normals provided the 3D catheter direction (Fig. 1b). CB model including an ellipsoid, marker, and catheter were constructed in consideration of the specifications of Arctic Front Advance ProTM (Medtronic, Minneapolis, USA). Since the CB model is rotationally

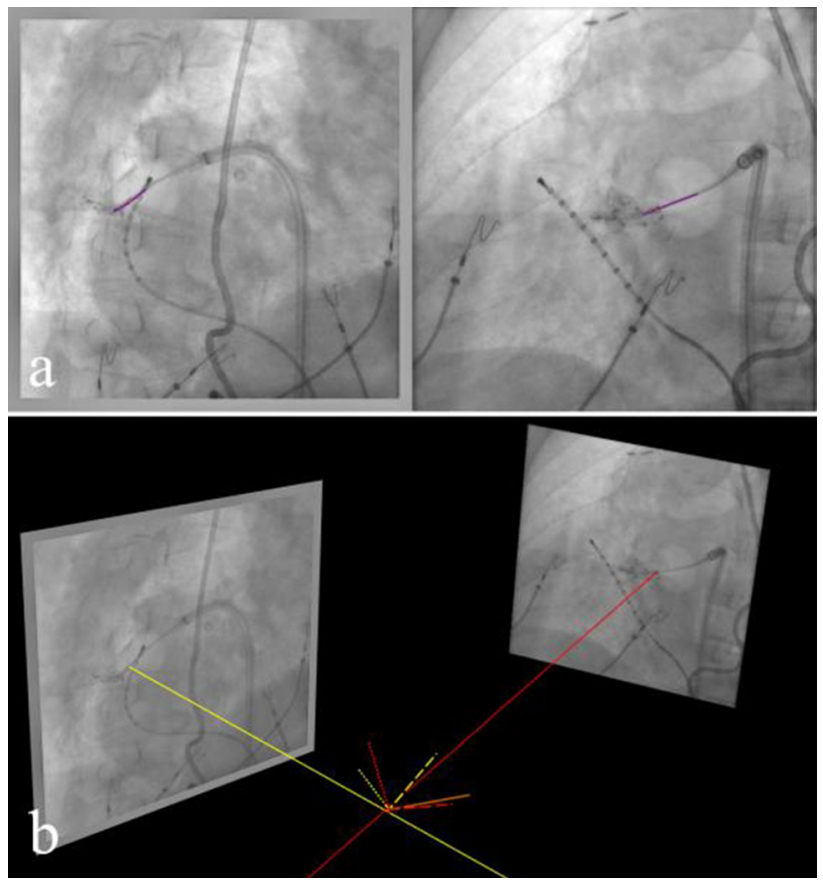

Fig. 1 a Biplane XR marked with automatically detected marker (orange), catheter shaft (blue), and fitted line (red). b From the projection vector (solid line) and the catheter orientation (dotted line), initialized by $3 \mathrm{~d}$ marker position (orange blob), the normal (dashed line) was determined for frontal (red) and lateral (yellow) view, yielding the $3 \mathrm{~d}$ catheter orientation (orange solid line) 


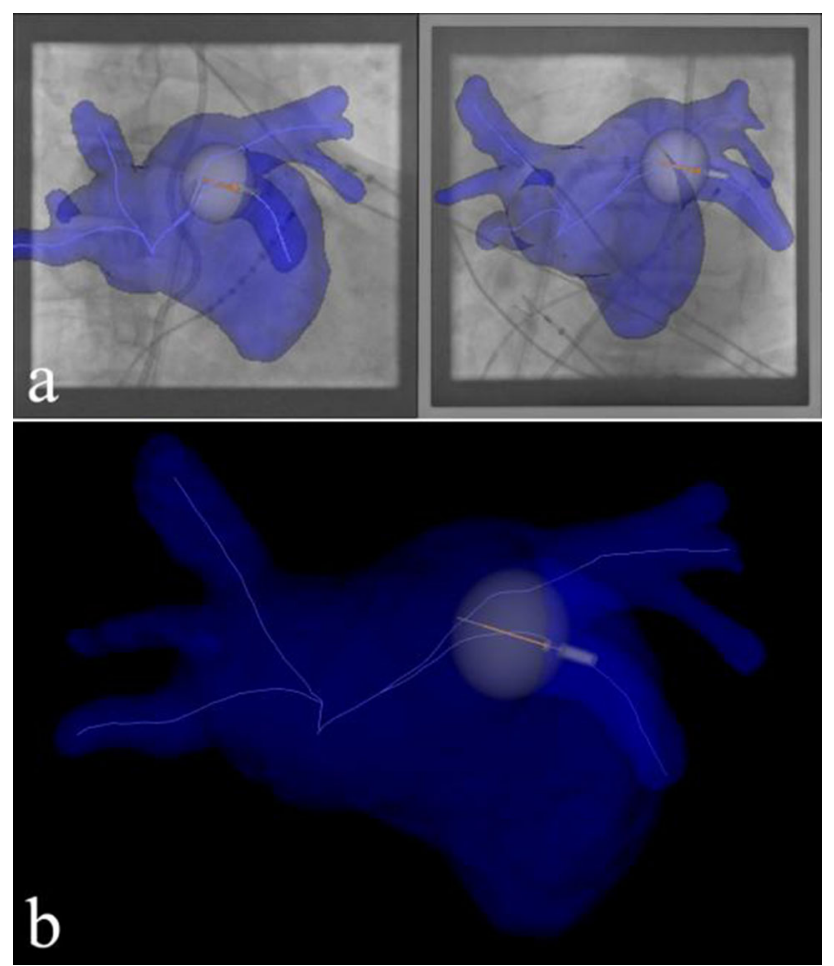

Fig. 2 CB model (yellow) aligned to the reconstructed marker and catheter shaft (orange) with respect to the LA model (blue) and its centerlines overlaid on the biplane XR (a) and as registered 3d model (b)

symmetric, there are only 2 landmarks required for proper 3D-3D registration of the $\mathrm{CB}$ model to the $\mathrm{XR}$ fluoroscopies. A paired-point registration between $\mathrm{CB}$ model and $\mathrm{XR}$ fluoroscopy was done using the marker center and $\mathrm{CB}$ center. Further, 3D CT-based surface models of the left atrium (LA) including the PV ostia were generated. Besides the surface model of the LA, its PV centerlines were generated. Co-registration of the LA model and XR angiography of the LSPV was performed manually.

\section{Results}

The automatic localization of the CB marker and catheter shaft using U-net could be achieved with high accuracy. The only limitation results from tricky catheter orientation and overlay of interfering structures, especially contrast agent injection. Figure 2 shows an example of the reconstructed catheter orientation and the aligned $\mathrm{CB}$ model as 2D overlays in a) and as 3D model b). As can be seen in Figure 2a, the model aligned to the reconstructed structures match the marker and catheter shaft in both biplane XR-projections. Further, the anatomic overlay enables the recognition of the relevant anatomy. By alignment of the $\mathrm{CB}$ model to the reconstructed orientation in combination with the registered 3D model of the LA and its centerlines, the position and orientation of the interventional tool within the patients" specific anatomy can be visualized (Fig. 2b).

\section{Conclusion}

The presented approach yields a visualization of the $\mathrm{CB}$ in relation to the patient specific target structure. Limitation of the detection method requires further work to ensure the robustness of the reconstruction algorithm, e.g. in single-frame detection failure or if opposite catheter orientation direction is detected. However, the automatic detection of $\mathrm{CB}$ structures is the basis on the way to realtime visualization. In an intraprocedural environment the $\mathrm{CB}$ position potentially might be verified or adjusted by alignment of the reconstructed catheter with the PV centerline. Therefore, the correct manual registration is essential. Further application may consist in documenting the intervention and subsequently pre-procedural planning in case of re-intervention. Further evaluation needs to be done regarding the deviation in case of highly angulated catheters. Nevertheless, the presented approach provides a basis for a simple procedure to likely facilitate correct $\mathrm{CB}$ positioning during $\mathrm{PV}$ ablation, which is expected to be further refined.

Acknowledgements

The project on which this report is based was funded by the Federal Ministry of Education and Research under the funding code 13GW0372C. Responsibility for the content of this publication lies with the author.

\section{References}

[1] Andrade JG, Dubuc M, Guerra PG, Macle L, Mondésert B, Rivard L, Roy D, Talajic M, Thibault B, Khairy P (2012) The biophysics and biomechanics of cryoballoon ablation. Pacing and clinical electrophysiology, 35(9):1162-1168

[2] Vernikouskaya I, Rottbauer W, Rasche V (2020) Real-time catheter tip segmentation and localization in 2D X-ray fluoroscopy using deep convolutional neural network. In CARS Proceedings 2020, Int J Comput Assist Radiol Surg 15(Suppl 1): S67-S69

\section{Model-based surgery-evaluation of preoperative characteristics}

T. Vogel ${ }^{1}$, M. Berlet ${ }^{1}$, D. Ostler ${ }^{1}$, M. Kranzfelder ${ }^{1}$, H. Feussner ${ }^{1}$, D. Wilhelm ${ }^{1}$

${ }^{1}$ Klinikum rechts der Isar, Technical University of Munich, MITIMinimally invasive Interdisciplinary Therapeutical Intervention,

Department of Surgery, Munich, Germany

Keywords cognitive surgery, prediction, surgery, workflow

\section{Purpose}

Cognitive surgery is the goal of extensive scientific efforts. This term includes, among other things, the possibility of predicting the course of certain steps during a surgical procedure. This implies two central aspects. First, it must be defined which steps a surgery consists of at all, and second, it must be defined which parameters are crucial to predict the course of a certain phase of the surgery. The assumption is therefore that there are parameters such as laboratory values and biometric measurements that influence the individual course. So the main problem is to find a way to define these appropriate parameters using statistical methods and to determine which combination is optimal here.

\section{Methods}

We selected the laparoscopic cholecystectomy as a representative, and daily performed procedure with a high degree of standardization, in order to find a procedure that is suitable to define crucial parameters. For this purpose, an existing database of 201 laparoscopic cholecystectomies was used. From each surgical phase, the duration of the dissection phase was determined and tested for the suitability of 19 parameters (age, height, sodium, potassium, weight, AP, bilirubin, GGT, GPT, GOT, albumin, CRP, Quick, PTT, leukocytes, gender, gallbladder stone size, platelets, and thickness of abdominal fat).

A "long" dissection was arbitrarily defined as one that lasts longer than 05 "33" " (median of the entire collective). The collective was initially divided into two groups $\mathrm{K} 1$ and $\mathrm{K} 2$. K1 was used to find 
suitable marker combinations, which were then to be validated with K2.

The definition was done in two steps. First, receiver operating characteristic (ROC) analysis was used to determine appropriate cutoff values for each marker, which were determined by optimizing the product of sensitivity and specificity. In a second step, the found cutoff values were combined by an R-script to evaluate which combinations of parameters could perform an even better prediction for a patient with a preparation time of more than $05^{\prime} 33^{\prime \prime}$ or. This was again done by optimizing the product of sensitivity and specificity, involving testing for combinations of one, two, or up to eight parameters. Tests were considered positive if half or more of the parameters suggested a longer dissection time. The constellations with the best product of sensitivity and specificity were then tested with the second collective K2.

Results

ROC analysis of the 19 parameters studied revealed intermediate sensitivities and specificities for several parameters, but none was higher than 74 and $72 \%$, respectively. Thus, no single parameter was suitable to predict with certainty whether the respective patient would have a longer preparation time.

When the parameters were combined with the cuttoff values found, the combination of the six values 'height $<177 \mathrm{~cm}$ ', 'sodium $>142 \mathrm{mmol} / \mathrm{l}$ ', 'potassium $<4.5 \mathrm{mmol} / \mathrm{l}$ ', 'body weight $>$ $80 \mathrm{~kg}$ ', 'AP $>66 \mathrm{U} / \mathrm{l}$ ', 'Quick $>105 \%$ ', and 'abdominal fat thickness $<4 \mathrm{~cm}$ ' was the optimal combination to separate patients with a long preparation time from those with a short one. (Sensitivity $88 \%$, Specificity $74.5 \%$ ).

The tests found in the optimization phase were then applied to the remaining $\mathrm{K} 2$ collective. Here, the optimal combination of the six parameters described above yielded a sensitivity of only 68 and a specificity of $55 \%$.

\section{Conclusion}

It is still difficult to define which parameters are crucial for an appropriate patient model in cognitive surgery. Here we describe an approach to this problem using the well-established method of ROC analysis. Since the separation of a patient collective with respect to gallbladder dissection time during laparoscopic cholecystectomy is improved when several parameters are combined, this method seems promising. However, it still requires some validation and optimization as well as testing in the context of other surgical procedures. There is an urgent need for valid patient models to advance cognitive surgery. Retrospective analysis of procedures already performed is an important means to design such models, and appropriate approaches are crucial.

\section{Magnetic nanoparticle detector for laparoscopic surgery: assessment of detection sensitivity}

M. Horstman—van de Loosdrecht ${ }^{1}$, L. Molenaar ${ }^{1}$, E. Krooshoop ${ }^{1}$, B. ten Haken ${ }^{1}$, J. Meijerink ${ }^{2}$, L. Alic ${ }^{1}$, I. Broeders ${ }^{3,4}$

${ }^{1}$ University of Twente, Magnetic Detection \& Imaging, Enschede, Netherlands ${ }^{2}$ Radboud University Medical Center, Operating Rooms and MITeC, Nijmegen, Netherlands ${ }^{3}$ Meander Medical Center, Surgery, Amersfoort, Netherlands ${ }^{4}$ University of Twente, Robotics and Mechatronics, Enschede, Netherlands

Keywords Magnetic detection, Laparoscopic surgery, Sentinel lymph node, Magnetic nanoparticle

\section{Purpose}

Sentinel lymph node biopsy (SLNB) is a minimally invasive procedure developed to detect and remove sentinel lymph nodes (SLNs) to select treatment regimes in a variety of tumor types. An SLN is one of the first lymph nodes draining from a primary tumor and it therefore

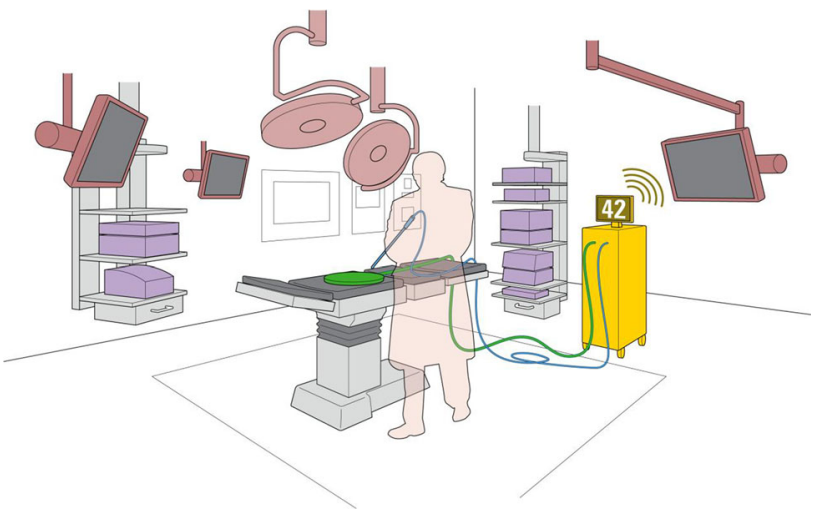

Fig. 1 LapDiffMag situated in an operating room. The excitation coil is shown in green, the detection probe in blue, and the control unit in yellow. The number (42) displayed, and the pitch of the sound represent either the amount of particles close to the probe or the distance between probe and sample

has the highest probability of containing metastases. The detected SLNs are surgically removed and pathologically investigated to identify potential metastases. Consequently, when identified during surgery and free of tumor cells, removing all regional lymph nodes becomes obsolete decreasing the burden to the patient. Magnetic SLNB is enabled by injection of superparamagnetic iron oxide nanoparticles (SPIONs) combined with a handheld probe detecting the SPIONs. Additionally, a pre-operative MRI scan can be used for intraoperative guidance and a post-operative MRI scan can be used to confirm that all SLNs were removed. For abdominal tumors, such as prostate cancer, (robot-assisted) laparoscopic surgery is standard care. The main challenge when developing a detector for a laparoscopic procedure is the combination of diameter of the detector (limited by the use of trocars) and sufficient detection depth. A laparoscopic differential magnetometer (LapDiffMag) was developed to enable magnetic laparoscopic SLNB, and assessed on its clinical performance regarding (depth) sensitivity.

\section{Methods}

To detect magnetic nanoparticles, differential magnetometry (DiffMag) was used [1]. This patented nonlinear detection method is SPION-specific and is influenced minimally by surrounding tissue and surgical instruments made of steel. LapDiffMag utilizes excitation coils to activate magnetic nanoparticles and detection coils to acquire the consequent magnetization of the particles. To maintain sufficient depth sensitivity after decreasing the diameter of the detector, the excitation and detection part of the system were separated [2]. Excitation coils were designed large and placed underneath the patient, while the detection coils in the probe are kept small enough to fit through a standard $12 \mathrm{~mm}$ trocar (Figure 1: setup). However, with this new setup, the detection coils move through the excitation field, leading to disturbances in SPION detection. This was solved by active compensation, a way to actively cancel-out the excitation field perceived by the detection coils, facilitated by an additional set of compensation coils [2]. To assess performance of the LapDiffMag, we used Magtrace ${ }^{\circledR}$ magnetic nanoparticles (a CE-certified and FDA-approved tracer for SLNB) in the following experiments:

- Identification of minimum iron content detectable by LapDiffMag: various amounts of Magtrace ${ }^{\circledR}(2.8,5.6,7,9.8,14,28,42$, $56,84,112,252$, and $504 \mu \mathrm{g}$ iron) were positioned directly in front of the probe. 
- Assessment of depth sensitivity of LapDiffMag: a sample containing $504 \mu \mathrm{g}$ iron was measured at various distances to the detection probe in air.

\section{Results}

An advantage of LapDiffMag is that it potentially can be used in a regular operating room during laparoscopic surgery, in contrast to other magnetic methods such as an MRI scan or EMG measurement. Strength of the signal detected by the LapDiffMag system is presented on a screen and as a sound with a pitch corresponding to the strength of the signal detected. The minimum detectable amount of iron by LapDiffMag was found to be $9.8 \mu \mathrm{g}$, representing the sensitivity of the system. Detection depth of LapDiffMag for a sample containing $504 \mu \mathrm{g}$ iron was found to be $10 \mathrm{~mm}$.

\section{Conclusion}

LapDiffMag demonstrated promising first results in terms of iron sensitivity and detection depth. It is a new route for laparoscopic magnetic SLNB that has the potential to facilitate abdominal cancer treatment strategies.

\section{References}

[1] Visscher M, Waanders S, Krooshoop H J G, ten Haken B (2014), Selective detection of magnetic nanoparticles in biomedical applications using differential magnetometry, Journal of Magnetism and Magnetic Materials, 365: 31-39

[2] van de Loosdrecht M M, Waanders S, Krooshoop H J G, ten Haken B (2018), Separation of excitation and detection coils for in vivo detection of superparamagnetic iron oxide nanoparticles, Journal of Magnetism and Magnetic Materials, 493: 165362

\section{Spatial information considered module based on attention mechanism for self-supervised depth estimation from laparoscopic image pairs}

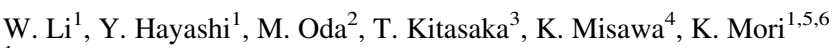
${ }^{1}$ Nagoya University, Graduate School of Informatics, Nagoya, Japan ${ }^{2}$ Nagoya University, Information Strategy Office, Information and Communications, Nagoya, Japan ${ }^{3}$ Aichi Institute of Technology, Faculty of Information Science, Toyota, Japan ${ }^{4}$ Aichi Cancer Center Hospital, Department of Gastroenterological Surgery, Nagoya, Japan ${ }^{5}$ Nagoya University, Information Technology Center, Nagoya, Japan ${ }^{6}$ National Institute of Informatics, Research Center of Medical Bigdata, Nagoya, Japan
}

Keywords Depth estimation, Laparoscopic surgery, Abdominal surgery, Self-supervised learning

\section{Purpose}

Recently, robotic surgical system has been developed as a powerful tool for assisting minimally invasive surgery. Depth estimation is useful to generate surgical navigation information in robot-assisted laparoscopic surgery system. Due to the development of deep learning and its wide application in depth estimation, the accuracy of depth maps predicted by self-supervised deep learning have been greatly improved by introducing a left-right depth consistency loss compared to traditional methods. However, continuous convolution operations in the feature encoder module lead to the loss of spatial information of input images and blur depth estimation at object boundaries estimated in depth images after feature decoder module. In order to maintain high-resolution representations, this paper proposes an attentionbased contextual module for self-supervised learning in depth estimation task.

\section{Methods}

The basic self-supervised strategy is followed by the previous method [1]. At the training phase, we input the rectified stereo image pairs, corresponding to the left and right laparoscopic color images, into the network. Then the left one is used to predict the stereo disparity maps. Based on the left color image and right disparity map, we reconstruct the fake right color image. While in a similar way, the left reconstructed image will also be predicted by the right color image and left disparity map. The depth maps are obtained from the stereo disparity maps using the extrinsic parameters of the stereo cameras. However, spatial information of original image is lost in the previous network [1] that is based on the standard architecture with chained convolution layers.

The proposed attention-based contextual module consists of three major blocks: the attention-based block, the dense atrous convolution (DAC) block and the spatial pyramid pooling (SPP) block. Generally, depth estimation is realized at pixel level by finding an accurate correspondence between image pairs under this self-supervised strategy. Therefore, a cross-dimension interaction attention-based block is adopted to learn contextual representations of features with similar semantic similarity at the first of this module. This attentionbased block is made up of three parallel branches. The first two branches build a channel attention mechanism together. The final branch is used to construct spatial attention mechanism by the channel pooling layer. Then, we introduce DAC block and SPP block into the proposed module.

These two blocks are both to reduce the loss of spatial information after chained convolution operations. DAC block contains four cascade branches with different number of atrous convolution that employs different receptive fields, and encodes the high-level feature maps. SPP is also adopted to compress features into four scales where the low-dimensional feature maps are up sampled to the same size as input feature map in final. We design the two parallel branches for these two blocks instead of a chained architecture in the previous method to reduce the loss of spatial information. We integrate these methods into a module to predict depth for medical images. Moreover, this module can be plugged into classic backbone network easily and the experiments show it can improve the performance of previous method [1].

\section{Results}

The proposed method was implemented in Pytorch. We used the stereoscopic image pairs dataset from the Hamlyn Centre Laparoscopic/Endoscopic Video Datasets [2]. This training dataset includes 18,624 rectified image pairs, and testing dataset includes 5231 rectified image pairs. They are both collected in partial nephrectomy using da Vinci robotic assisted system with stereo-laparoscope. We performed an ablation study by changing components of our module. The experimental result showed that combination of all components leads to improve performance. Moreover, we replaced the backbone in the previous method with the U-Net and repeat the experiments to evaluate the performance of the proposed module on different backbones. And the proposed module leaded to an improvement on different backbones.

The mean SSIM and PSNR of testing stereo image pairs were shown in Table 1. Our experimental results showed that the proposed module improved about $5.25 \%$ in PSNR and about $2.72 \%$ in SSIM, comparing with the previous method [1]. Meanwhile, the spatial information of the input image was retained with chained convolution layers, as shown green boxes in Fig. 1.

\section{Conclusion}

In this work, we proposed a novel attention-based contextual module for self-supervised depth estimation method from stereo laparoscopic images. This module tried to tackle the limitation of previous method that consecutive convolution operations that lead to a low-representation and loss of spatial information of images. The module is made 
Table 1 Comparison of proposed module on different backbone networks by SSIM and PSNR

\begin{tabular}{lllll}
\hline Methods & $\begin{array}{l}\text { Mean SSIM } \\
\text { (Left) }\end{array}$ & $\begin{array}{l}\text { Mean SSIM } \\
\text { (Right) }\end{array}$ & $\begin{array}{l}\text { Mean PSNR } \\
\text { (Left) }\end{array}$ & $\begin{array}{l}\text { Mean PSNR } \\
\text { (Right) }\end{array}$ \\
\hline $\begin{array}{l}\text { Baseline [1] } \\
\begin{array}{l}\text { Baseline } \\
\text { (DAC + SPP) }\end{array}\end{array}$ & 0.85 & 0.84 & 18.3 & 18.08 \\
$\begin{array}{l}\text { Baseline } \\
\text { (full module) }\end{array}$ & $\mathbf{0 . 8 7}$ & 0.85 & 19.16 & 18.8 \\
$\begin{array}{l}\text { U-Net } \\
\begin{array}{l}\text { U-Net } \\
\text { (DAC + SPP) }\end{array}\end{array}$ & 0.84 & $\mathbf{0 . 8 6}$ & $\mathbf{1 9 . 3 4}$ & $\mathbf{1 8 . 9 4}$ \\
$\begin{array}{l}\text { U-Net } \\
\text { (full module) }\end{array}$ & $\mathbf{0 . 8 6}$ & 0.83 & 17.66 & 17.58 \\
\hline & 0.84 & 18.13 & 18.26 \\
\hline & & $\mathbf{0 . 8 6}$ & $\mathbf{1 8 . 6 7}$ & $\mathbf{1 8 . 9}$ \\
\hline
\end{tabular}

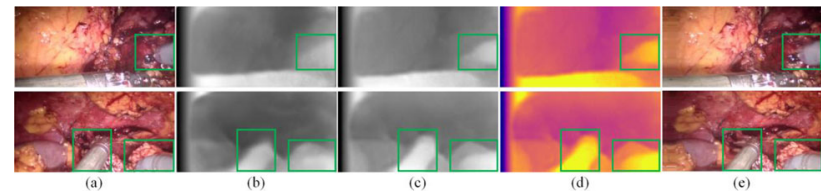

Fig. 1 Example and comparison of previous method [1] and proposed method. a original left image of stereo image pairs, b disparity maps estimated by the previous method, c disparity maps estimated by the previous with the proposed module, $\mathbf{d}$ disparity maps with color maps, and e reconstructed left image. Green boxes indicate the results of the previous method with and without the proposed module in the same area

up of three blocks. The attention-based block is chosen according to the consideration of high accuracy requirement for depth estimation at the pixel level. DAC and SPP are designed into two parallel branches instead of traditional chain structure. Experimental results showed that the proposed module improved the accuracy of the disparity map and retained the spatial information of the input image compared with the previous method.

\section{References}

[1] Clément G, Aodha O, Brostow G (2017) Unsupervised monocular depth estimation with left-right consistency. Proceedings of the IEEE Conference on Computer Vision and Pattern Recognition: 270-279

[2] Ye M, Johns E, Handa A, Zhang L, Pratt P, Yang GZ (2017) Self-supervised siamese learning on stereo image pairs for depth estimation in robotic surgery. Hamlyn Symposium on Medical Robotics

\section{Real-time deformation simulation of hollow organs based on XPBD with small time steps and air mesh for surgical simulation}

\author{
S. $\mathrm{Li}^{1}$, Y. Hayashi ${ }^{1}$, M. Oda ${ }^{2}$, K. Misawa ${ }^{3}$, K. Mori ${ }^{1}$ \\ ${ }^{1}$ Nagoya University, Graduate School of Informatics, Nagoya, Japan \\ ${ }^{2}$ Nagoya University, Information Strategy Office, Information \\ and Communications, Nagoya, Japan ${ }^{3}$ Aichi Cancer Center, \\ Department of Gastroenterological Surgery, Nagoya, Japan
}

Keywords Real-time, Deformation simulation, Hollow organs, Surgical simulation

\section{Purpose}

With the development of computer technologies, surgical simulator is gradually utilized for the surgical skill training. Since most organs of the human body are deformable, the deformation simulation of them is fundamental to a surgical simulator. For providing high-quality user experience, the simulation also needs to be real-time. Although some research has been carried out on this topic, there are few studies that are focused on hollow organs. Target organs in organ deformation simulation studies are commonly solid, such as the liver and kidneys, or hollow but treated as solid, such as the gallbladder. Obviously, the deformation of hollow organs may not be simulated in the same way. Therefore, in this paper, a fast, simple, and robust method is proposed to simulate the deformation of hollow organs in real-time. We proposed a novel double-layer model to simulate hollow organs deformation and adopted extended position-based dynamics (XPBD) with small time steps [1] to simulate deformation and air mesh [2] to prevent self-collision. We tested our method on a stomach model.

\section{Methods}

Double-layer model

A general organ model usually consists of only a single-layer surface mesh without thickness. To obtain the volume mesh, which is necessary for the simulation, all the inner space is tessellated and assumed solid regardless of whether the organ is solid or not in reality. For more appropriate simulation of hollow organs, a hollow model would be better instead of a solid one. Therefore, we proposed a novel double-layer hollow model. By increasing the thickness of the surface in a single-layer model, we obtained a double-layer model that consists of an outer surface and an inner surface. The space inside the inner surface represents the hollow structure and the space between the outer and inner surfaces represents the wall of hollow organs. We tetrahedralized the "wall" part to get the volume mesh for deformation simulation. The "hollow" part was also tetrahedralized, which is called air mesh and will be explained in later paragraphs.

Basic deformation simulation

XPBD with small time steps [1] is popular in physical simulation recently. It can provide robust and visually plausible deformation in real time, which is crucial in surgical simulation. Furthermore, it is also extensible by adopting different types of constraints to achieve various effects. Therefore, we adopted XPBD with small time steps as our deformation simulation framework. We built distance constraints along each edge in the volume mesh of the "wall" part in our model, which can conserve the distance between two vertices of one edge. To conserve the volume of organ, we also gave each tetrahedron in the "wall" part a signed volume constraint. Compared with general volume constraint, signed volume constraint prevents tetrahedrons from inversion naturally, which is common when the model is severely distorted. XPBD with distance constraint and signed volume constraint enables to perform the basic deformation simulation of hollow organs.

Self-collision prevention

Along with the double-layer model, self-collision prevention method is also introduced. The self-collision refers to the collision between different parts of the model itself. In solid organ models, self-collision is rare due to the solid shape. However, since there are no constraints inside the hollow interior space in a double-layer model, self-collision will occur. Therefore, we introduced air mesh [2] to solve this problem. The "hollow" part was tetrahedralized and called air mesh. We add unilateral volume constraints to tetrahedrons in the air mesh and integrated it into the XPBD framework. During the simulation, when the volume of a tetrahedron in the air mesh is negative which means it is inverted, unilateral volume constraint makes its volume back to zero. The self-collision problem is solved robustly and naturally in this way without increasing too much computational burden. 


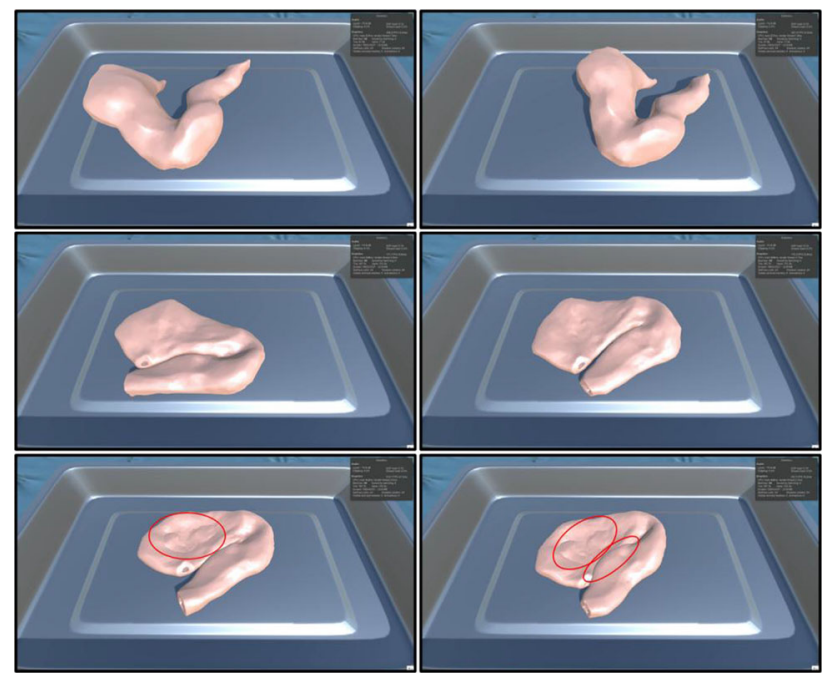

Fig. 1 The simulation results. The upper two images are the result of single-layer solid model. The middle two images are the result of double-layer hollow model with air mesh. The lower two images are the result of double-layer hollow model without air mesh. The red circles denote the self-collision parts

\section{Results}

We tested our method on a PC with Intel Core i7-8700 K CPU, NVIDIA GeForce RTX 3080 GPU, and 64.0 GB RAM. We generated a stomach model from stomach region segmented from a CT volume. The processed double-layer stomach model has 10,316 vertices, 35,424 tetrahedrons in volume mesh, and 33,315 tetrahedrons in air mesh. Unity was chosen as our engine because it facilitates rapid development. We let the model free fall onto a frictionless tray and simulated the deformation. The deformation simulation and selfcollision prevention procedures were computed parallelly on GPU. The average processing time per frame was $5.65 \mathrm{~ms}$. We also compared the results of single-layer solid model, double-layer hollow model with air mesh, and double-layer hollow model without air mesh. The comparison is shown in Fig. 1. Compared with the simulation of a single-layer solid model, the simulation of a double-layer hollow model is more plausible and closer to the real deformation of a hollow organ. Compared with the simulation without air mesh, the simulation with air mesh solves the self-collision problem well.

\section{Conclusion}

This paper aims to simulate the deformation of hollow organs in real time for the further application in surgical simulation. We proposed a fast, simple, and robust method based on a novel double-layer model, XPBD with small time steps, and air mesh. We tested our method with a stomach model and achieved a favorable result. A limitation of this work is that constraints of the deformation simulation are too simple to show the complex physical properties, like anisotropy and heterogeneity. We will work on it in future.

\section{References}

[1] Macklin M, Storey K, Lu M, Terdiman P, Chentanez N, Jeschke S, Müller M (2019) Small steps in physics simulation. SCA'19: Proceedings of the 18th annual ACM SIGGRAPH/Eurographics Symposium on Computer Animation

[2] Müller M, Chentanez N, Kim T-Y, Macklin M (2015) Air Meshes for Robust Collision Handling. ACM Trans Graph 34:133:1-133:9. https://doi.org/10.1145/2766907
Active positioning arm with high RCM changeability using parallel sliding mechanism for minimally invasive surgery

Y. $\mathrm{Yi}^{1}$, B. S. Cheon ${ }^{2}$, S. Cho ${ }^{1}$, D. S. Kwon ${ }^{1,3}$

${ }^{1}$ Korea Advanced Institute of Science and Technology, Mechanical Engineering, Daejeon, South Korea ${ }^{2}$ Korea Advanced Institute of Science and Technology, Robotics Program, Daejeon, South Korea ${ }^{3}$ EasyEndo Surgical Inc., Daejeon, South Korea

Keywords Active positioning arm, Minimally invasive surgery, Parallel sliding mechanism, RCM Changeability

\section{Purpose}

Minimally Invasive Surgery (MIS) robot systems have a remote center of motion (RCM) in which a surgical instrument conducts a fulcrum motion around a point of incision. It is generally considered that there is no lateral motion to RCM point, however, in real surgery, there are many cases in MIS the RCM point moves. For example, the RCM point may be moving because of heavy breathing, strong heartbeat, and movement of the patient [1]. Moreover, the RCM point is changed to get additional sight of the lesion in arthroscopic surgery and vitreoretinal surgery.

Many active positioning arms (APAs) for RCM have been developed over many years and can be classified into two categories, depending on whether the RCM point is defined and mechanically locked based on the kinematics of the mechanism (e.g. link) or not [2]. APAs that the RCM point is mechanically locked are strong against external forces that are related to safety. However, it is difficult to set these arms to move RCM point while preventing interference among robot arms or between robot arms and surgeons. This is because the more change of inertia makes the larger workspace for moving RCM point. On the other hand, APAs that control virtual RCM point without any kinematic constraints have an advantage to freely move RCM point, but they are vulnerable to external forces.

It is necessary for APA to have a strong structure against external forces but move RCM point with the less change of inertia of APA. In this paper, we propose a simple but novel APA using parallel sliding mechanism for MIS. This mechanism makes 2-degree of freedom (pitch rotation and translation) by the relative motion of sliders so that the proposed APA has a strong structure but is able to move RCM point with the less change of inertia.

This work was supported in part by the National Research Foundation of Korea through the Framework of International Cooperation Program under Grant 2019K2A9A2A06025429 and Grant FY2019, in part by the Korea Medical Device Development Fund grant funded by the Korea Government (the Ministry of Science and ICT, the Ministry of Trade, Industry and Energy, the Ministry of Health \& Welfare, Republic of Korea, the Ministry of Food and Drug Safety) under Project 202012D18.

\section{Methods}

The proposed APA has one motor for roll, two motors for parallel sliding mechanism, and one motor for translation of instrument. The upper slider and lower slider move independently in parallel along with the upper and lower ball screws respectively. Both sliders are connected with the link as shown in Fig. 1.

The kinematics for RCM roll rotation is not dealt with in method section because the proposed APA has the same kinematics as other mechanisms.

1. Kinematics and control method of parallel sliding mechanism The control variables for parallel sliding mechanism in this APA are $X_{1}$ and $X_{2}$ which are the distance the lower and upper slider move respectively as shown in Fig. 1. We can easily show the 


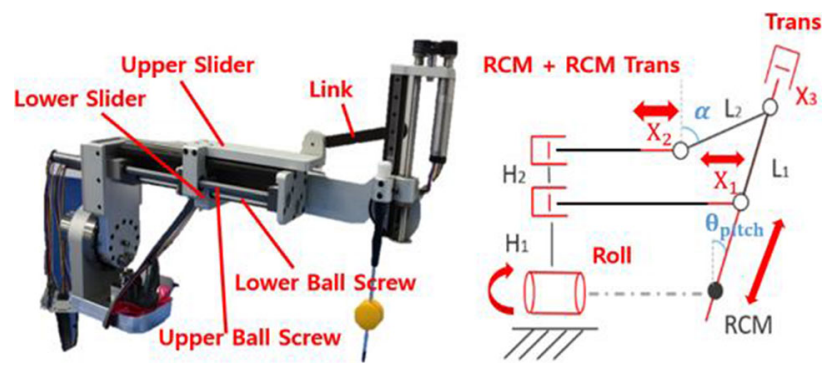

Fig. 1 Developed active positioning arm using parallel sliding mechanism for MIS and its kinematic chain

kinematics of parallel sliding mechanism for RCM pitch rotation as follows:

$\mathrm{X}_{1}=\mathrm{H}_{1} \tan \left(\theta_{\text {pitch }}\right),\left(\theta_{\text {pitch }} \neq 0\right)$

$\mathrm{X}_{2}=\mathrm{X}_{1}+\mathrm{L}_{1} \sin \left(\theta_{\text {pitch }}\right)+\mathrm{L}_{2}\left(\sin \left(\alpha_{0}\right)-\sin (\alpha)\right)$

where $\alpha=\cos ^{-1}\left(\left(\mathrm{~L}_{1} \cos \left(\theta_{\text {pitch }}\right)-\mathrm{H}_{2}\right) / \mathrm{L}_{2}\right) \quad$ and $\theta_{\text {pitch }}$ is the angle change of tool in RCM pitch rotation. $\alpha_{0}, \mathrm{H}_{1}, \mathrm{H}_{2}, \mathrm{~L}_{1}, \mathrm{~L}_{2}$ are all constant and these constants are determined considering the environment of operation and workspace.

There are two control methods in parallel sliding mechanism, one is RCM pitch rotation and the other is RCM translation. For RCM pitch rotation, we control $X_{1}$ and $X_{2}$ following the above equation (1) and (2). Relative motion between 2 sliders $\left(\theta_{\text {pitch }} \neq 0, X_{1} \neq X_{2}\right)$ makes RCM pitch rotation. For RCM translation, we give the same input to the $X_{1}$ and $X_{2}\left(X_{1}=X_{2}, X_{1} \neq 0\right)$. Then, there is no change of $\theta_{\text {pitch }}$ and it makes only translation of RCM point.

2. RCM Changeability

To evaluate quantitively how efficiently APA changes RCM point, we suggest a new criterion, RCM Changeability.

RCM Changeability = Displacement of RCM point/Displacement of COM of active positioning arm

RCM changeability means that how much APA moves to move RCM point. The inertia change of APA determines the energy to move APA, interference among APAs. The change of inertia is related to the displacement of the center of mass (COM) so that the equation of RCM changeability consists of the displacement of RCM point and the displacement of COM of APA.

Results

In this section, we compare the double parallelogram mechanism which most of APAs use and the proposed parallel sliding mechanism with RCM changeability. We only consider the one-dimensional movement of RCM point and COM assuming the kinematics of RCM roll rotation, the total mass of APA and workspace of APA is the same.

Let the movement of RCM point is $\Delta \mathrm{x}$. For the double parallelogram mechanism, the movement of COM and RCM point is the same because the entire APA has to be moved. Consequently, the RCM changeability is 1 . In contrast to the double parallelogram, only part of the proposed APA moves to change RCM point. The ratio of the mass of moving part to total mass is 0.236 so that movement of $\mathrm{COM}$ is $0.236 \times \Delta \mathrm{x}$. Therefore, $\mathrm{RCM}$ changeability of parallel sliding mechanism is 4.237 .

\section{Conclusion}

This paper proposed APA for MIS that can move RCM point by using the parallel sliding mechanism. We validated the proposed mechanism based on RCM changeability and the result shows that the parallel sliding mechanism change less inertia of APA to move RCM point than the double parallelogram mechanism.

\section{References}

[1] Pham CD, Coutinho F, Leite AC, Lizarralde F, From PJ, Johansson R (2015) Analysis of Moving Remote Center of Motion for Robotics-Assisted Minimally Invasive Surgery. IEEE/RSJ International Conference on Intelligent Robots and Systems (IROS)

[2] Kuo CH, Dai JS, Dasgupta P (2012), Kinematic design considerations for minimally invasive surgical robots: an overview. The International Journal of Medical Robotics and Computer Assisted Surgery; 8:127-145

\section{Needle puncture analysis using audio excitations during tissue insertions}

M. Sabieleish ${ }^{1}$, K. Heryan ${ }^{2}$, A. Boese ${ }^{1}$, M. Friebe ${ }^{1}$, A. Illanes ${ }^{1}$

${ }^{1}$ Otto-von-Guericke Universität, Medical Faculty, INKA-Innovation Laboratory for Image Guided Therapy, Magdeburg, Germany ${ }^{2} \mathrm{AGH}$ University of Science and Technology, Department of Measurement and Electronics, Kraków, Poland

Keywords needle puncture, audio guidance, audio processing, needle interventions

\section{Purpose}

Accurate percutaneous needle tip localization is essential during minimally invasive procedures (MIPs). Usually, less experienced surgeons face more complications during MIP-related interventions and have more difficulties localizing the needle tip. Biological punctures created during needle insertion procedures could deliver valuable information that can be used to localize the needle tip. Various imaging technologies are used to enhance accuracy and support the clinician. However, these technologies face limitations, such as image artifacts affecting the procedure's accuracy and efficacy. Increasing the accuracy of image-based needle guidance could be achieved with complementary sensors to provide additional guidance information. However, most of these specialized sensors are placed on the needle tip, resulting in direct contact with the biological tissue leading to increased risks, complexity, costs, and sterilization issues.

An audio-based technique has recently been introduced [1], showing promising results for different applications. However, the relationship between soft tissue events created by the needle tip and the audio signal excitation is still not well understood. A first study has been performed [2] to better understand audio excitation using force as a reference. It showed that force and audio dynamics are strongly related during needle insertion.

This work aims to study the factors that can influence and affect the audio signals recorded during a needle puncture and verify if tissue-related audio dynamics can be identified. One of the main challenges for audio event characterization is understanding the transfer function (TF) between the audio wave generated from the needle tip/tissue interaction at the contact zone and the received audio signal at the tool's proximal end. This work proposes a study of the dynamics related to the TF and punctures to analyze tissue-related audio dynamics' identifiability.

\section{Methods}

An automatic experimental setup for needle insertions was created to acquire the audio signals. A microphone attached to the proximal end of a short bevel steel rod ( $1.6 \mathrm{~mm}$ diameter, $140 \mathrm{~mm}$ length, $45^{\circ}$ tip) via a $3 \mathrm{D}$ printed adapter was used for recording. The rod simulates a simplified needle to perform insertions in two plastic tissues of different thicknesses. Two layers of each plastic were fixed in a 3Dprinted tissue holder immersed in a gelatin phantom at a depth of $3 \mathrm{~cm}$ and $5.5 \mathrm{~cm}$ the first and second layers, respectively. The 
insertions were performed automatically using a testing machine (Zwicki, Zwick GmbH \& Co.KG, Ulm) at an insertion velocity of $5 \mathrm{~mm} / \mathrm{s}$ that recorded the axial needle insertion force. The audio and force frequencies sampling was $16000 \mathrm{~Hz}$ and $100 \mathrm{~Hz}$, respectively. The signals were synchronized using a simultaneous trigger event visible in both the force and audio signals.

The setup aimed to test the effect of the TF modification on the audio signal by adding weights to the needle shaft and subsequently analyzing the impact of the tissue type on the audio excitation. Moreover, the setup was used to test the influence of puncture depth on audio dynamics.

For signal analysis, a dataset of 20 recordings/tissue/needle setup was generated. Continuous Wavelet Transformation (CWT) was used. As shown in Fig. 1, two main features were extracted from the CWT of the audio excitation resulting from a needle puncture: the frequency at which the maximum energy event occurs (Max.time-freq energy) and the dominant frequency observed in the normalized CWT spectrum that can be extracted at each time instant (see the last row of Fig. 1). Our goal was to observe the influence of the TF modification and a puncture excitation when different tissue types are punctured at different depths.

\section{Results}

Table 1 shows the average and the standard deviation values for the Max.time-freq energy feature for different insertions. It is possible to observe that the thicker tissue (tissue (2) produces higher maximal frequency responses than the thinner one. We can also observe that the TF modification strongly affects the extracted maximal frequency feature for both tissues. Additionally, another important observation is that this feature is not affected by the depth of the layer. For both tissues, the maximal frequency energy feature does not significantly change during the first and second punctures (layers 1 and 2, respectively).

Figure 2 shows the dominant frequency analysis results. Each row of the displayed matrix represents the probability distribution of each needle insertion's dominant frequencies. This feature"s behavior is similar to the previously analyzed feature. The modification of the TF results mainly in the apparition of new frequency dynamics around the frequency scale 45 . For both tissues, there are no significant changes between the first and second punctures.

The final fundamental observation is that when the TF is fixed, the dominant frequency behavior depends on the tissue punctured and not on the depth. This is very important since it demonstrates that it is

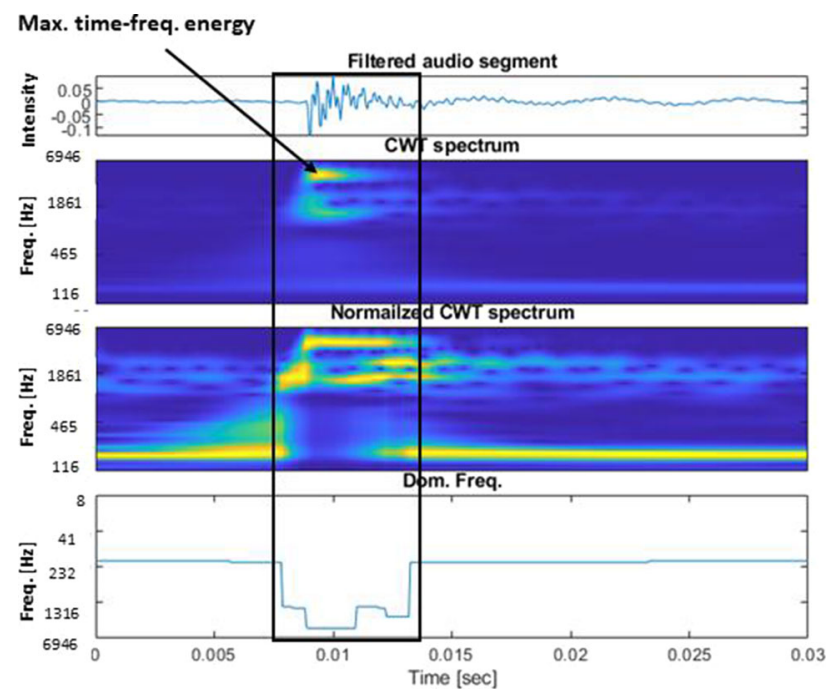

Fig. 1 Example of a puncture event (rectangle) and the computed CWT illustrating the extracted features
Table 1 Max.time-freq energy mean and standard deviation (SD) for all setups

\begin{tabular}{lc}
\hline Data & Mean \pm Std $[\mathrm{Hz}]$ \\
\hline 1st tissue, 1st puncture, One weight & $730.5 \pm 6.9$ \\
1st tissue, 1st puncture, Three weights & $650 \pm 10.7$ \\
1st tissue, 2nd puncture, One weight & $750 \pm 6.9$ \\
1st tissue, 2nd puncture, Three weights & $550 \pm 13.6$ \\
2nd tissue, 1st puncture, One weight & $800 \pm 5.2$ \\
2nd tissue, 1st puncture, Three weights & $1069 \pm 0.8$ \\
2nd tissue, 2nd puncture, One weight & $700 \pm 6.2$ \\
2nd tissue, 2nd puncture, Three weights & $1000 \pm 5.9$ \\
\hline
\end{tabular}
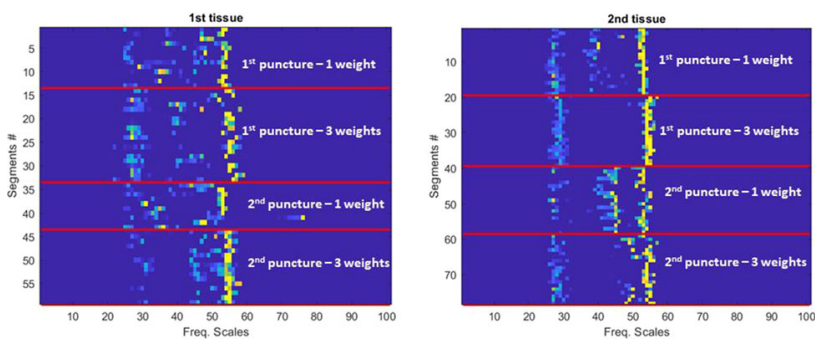

Fig. 2 Dominant frequency analysis results for both tissues during needle insertion

possible to identify tissue puncture-related dynamics for automatic insertions. Nevertheless, any modification on the TF could limit tissue puncture identification.

\section{Conclusion}

An analysis of different dynamics involved in the audio excitation during the needle insertion process was performed in this work. The most important conclusion of this work is that it is possible to identify tissue-related dynamics under a fixed TF and differentiate between two punctured tissues. These results show that needle audio guidance could be possible for automatic needle insertion.

\section{References}

[1] Illanes A, Sühn T, Esmaeili N, Maldonado I, Schaufler A, Chen CH, Boese A, Friebe M (2019) Surgical Audio Guidance SurAG: Extracting Non-Invasively Meaningful Guidance Information During Minimally Invasive Procedures. In 2019 IEEE 19th International Conference on Bioinformatics and Bioengineering (BIBE) (pp. 567-570). IEEE.

[2] Illanes A, Boese A, Friebe M, Hansen C (2020) Feasibility Check: Can Audio Be a Simple Alternative to Force-Based Feedback for Needle Guidance?. In International Conference on Medical Image Computing and Computer-Assisted Intervention (pp. 24-33). Springer, Cham. 


\section{Passive needle tracking using phase imaging for MR- guided interventions}

A. Mahran ${ }^{1,2}$, P. Moreira ${ }^{1,2}$, K. Tuncali ${ }^{1,2}$, J. Tokuda $^{1,2}$

${ }^{1}$ Brigham and Women's Hospital, Radiology, Boston, United States

${ }^{2}$ Harvard Medical School, Boston, United States

Keywords Device tracking, Interventional MRI, Needle detection, MRI guided intervention

\section{Purpose}

Percutaneous interventions under intraoperative magnetic resonance imaging (MRI) guidance have been used in various applications such as aspiration, biopsy, and thermal ablations [1]. Intraoperative MRI is useful in identifying target sites thanks to its superior soft-tissue contrast, and locating the needle with respect to the target site in near real-time based on the susceptibility artifact at the needle on the image. The ability to visualize both the target site and needle simultaneously potentially allows closed-loop control of the needle to achieve accurate placement of the needle into the target site using imaging guidance software and/or a needle-guiding robot. However, distinguishing the intensity generated by the susceptibility artifact at the needle is challenging to perform in real-time MRI. There have been two categories of approaches to address this technical challenge, including active and passive tracking [2]. Active tracking localizes the needle using a small MR receiver coil or additional sensors fitted to the needle. Although active tracking provides robust and accurate localization, it requires specialized hardware and additional safety considerations associated with it. Passive needle tracking, in contrast, localizes the needle by detecting image features, e.g. the needle artifact, bright spots produced by MR-visible markers. While passive tracking does not require specialized hardware, automatic detection of image features is not always reliable due to their inconsistent appearances on the image. In this study, we hypothesize that the MRI phase information can yield more distinct and consistent features around the needle than the commonly-used magnitude image, because of its direct correlation with local magnetic homogeneity; hence it helps to improve the accuracy of passive needle tracking. The phase image can be obtained as part of the standard scan output and therefore is available at no extra acquisition time or change in scanning parameters. This study proposes a new approach to passive device tracking by generating positive-contrast images from MRI phase images.

\section{Methods}

We implemented and evaluated the real-time MRI-based needle tracking algorithm during insertions into biological tissues. The algorithm uses a multi-step approach described in Fig 1, such as: (1) A mask is generated from the magnitude image to eliminate the noisy background in the phase images, (2) the phase image is unwrapped, (3) a high pass filter is applied to increase the signal-to-noise ratio in the background field, and (4) A hessian matrix is used for the needle tip localization.

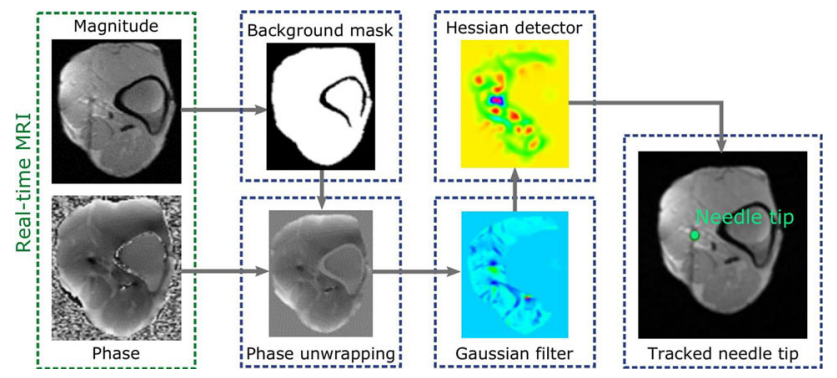

Mask Generation: It is known that areas with large susceptibility (i.e. air-tissue interfaces) are prone to artifacts because phase image measures the weighted summation of the magnetic properties of the surrounding tissue. A binary threshold was used in the magnitude image to mask out the areas with high susceptibility borders in the phase image. This eliminates the noisy phase in the air and bone surrounding the phantom and reduces computational time.

Phase Unwrapping: The original phase signal is encoded in such a way that it can only measure phase values in the range of $[-\pi,+2 \pi]$ causing the signal to fold over in the opposite direction. This wrapping operation makes the signal difficult to interpret because of the random $2 \pi$ jumps. Phase unwrapping is a process used to recover the continuous phase values from the wrapped one by detecting phase jumps and correctly adding appropriate $2 \pi$ radians. In the proposed tracking algorithm, the phase unwrapping is achieved using the Phase Region expanding labeler for Unwrapping Discrete Estimates (PRELUDE) technique.

Estimation and Removal of Background Field: Further filtering is needed to increase signal-to-noise ratio and reduce artifacts. We implemented a Gaussian high pass filter which serves two functions: it helps remove background field inhomogeneities since they have low spatial frequencies and enhance field inhomogeneity produced by the needle tip. The implemented Gaussian high pass filter has the following equation:

$\mathrm{H}(\mathrm{u}, \mathrm{v})=1-\mathrm{e}^{-\mathrm{D}^{2}(\mathrm{u}, \mathrm{v}) / 2 \mathrm{D} 0^{2}}$

where with a kernel size of $128 \times 128$ and the cut off distance $\mathrm{D}_{0}$ experimentally tuned to 5 .

Detecting Needle Tip position: To detect the needle tip from the processed image, A blob detector is employed based on the eigenvalue of the hessian matrix. The advantage of the hessian process is the ability to delineate the high intensity clusters. After the filter is passed, the needle tip is selected based on the peak local maxima.

Experimental Protocol: In this study, we preformed needle insertion experiments to validate the tracking accuracy and computational time of our proposed method. A MRI-compatible 18-Gauge biopsy needle was repeatedly inserted and retracted linearly with varying speed along an ex vivo tissue. Magnitude and phase images were acquired using a T2-weighted gradient echo sequence $(5 \mathrm{~mm}$ slice thickness, FOV of $300 \times 300 \mathrm{~mm}$, and $256 \times 128$ matrix). The tracking algorithm was implemented in 3D Slicer. The algorithm was evaluated using Sagittal (110 frames) and Coronal (110 frames) images acquired in different needle insertion sequences. The needle tip was tracked in all 220 image frames and the results were compared to the needle locations manually tracked offline using the magnitude images. The root mean square error (RMSE) between the needle tip locations tracked by the algorithm and the one manually tracked and the mean computational time required to track the needle in each image frame were calculated.

\section{Results}

The root mean square error of the automated needle tip compared to the manually segmented needle tip was $1.88 \mathrm{~mm}$ on average. Despite the coronal slices containing bone tissue, which causes a strong susceptibility artifact, the algorithm was able to consistently track the needle tip. The discrepancy between the algorithm and the manual tracking is within the expected range due to the limitations of manually segmenting the needle tip, such as artifact size and visibility of the needle tip in some frames. The average computational time was $0.72 \mathrm{~s}$. It is worth noting that the computation time was mostly due to the phase-unwrapping step and conversion of data format due to the reliance on the multiple image processing library; we expect that those steps could be potentially eliminated or optimized in the future implementation to achieve a shorter computational time.

Fig. 1 Workflow of needle tip detection 


\section{Conclusion}

We demonstrated the use of phase imaging for MR guided device tracking. Our method was able to consistently detect the needle tip without any change in the scan parameters or additional input from the operator. We are currently integrating this method with a closedloop scan plane control module. The proposed system will allow the information from the device tracking to be used directly to re-adjust the scan plane position and orientation to follow the needle trajectory autonomously.

\section{References}

[1] Campbell-Washburn AE, Faranesh AZ, Lederman RJ, Hansen MS (2015) MR sequences and rapid acquisition for MR guided interventions. Magnetic resonance imaging clinics of North America, 23(4), 669

[2] Bock M, Wacker FK (2008) MR-guided intravascular interventions: techniques and applications. Journal of Magnetic Resonance Imaging: An Official Journal of the International Society for Magnetic Resonance in Medicine, 27(2), 326-338

\section{Design of a human-machine interface between sterile and non-sterile environment of the OR for the usage of a robotic circulating nurse}

\section{Müller ${ }^{1}$, L. Bernhard ${ }^{1}$, D. Ostler ${ }^{1}$, D. Wilhelm ${ }^{1,2}$}

${ }^{1}$ Research Group MITI, Klinikum rechts der Isar, Technical University Munich, Munich, Germany ${ }^{2}$ Technical University Munich, Faculty of Medicine, Department of Surgery, Klinikum rechts der Isar, Munich, Germany

Keywords User interface, Circulating nurse, Operating room, Robotic

\section{Purpose}

This extended abstract presents the work-in-progress of a research project whose overall goal is the integration of a Robotic Circulating Nurse (RCN) into the operating room (OR) environment. Specifically, a human-machine interface is to be developed for this purpose, which includes the communication of the sterile OR staff with the environment. It should fit as seamlessly as possible into the surgical workflow, which means it should not complicate communication.

Within the AURORA project of the MITI research group of the TUM, a concept of a robotic assistant in the non-sterile OR environment is being developed, which takes over tasks of a circulating nurse to reduce the workload of the staff. The so-called circulating nurse $(\mathrm{CN})$ is non-sterile OR staff who has an assistant function and whose responsibilities include handing out sterile items and operating medical devices.

Concepts of robotic OR nurses for handling surgical instruments were among others examined in [1]. However, robotic surgical nurses, which have been developed so far all are located in the sterile area and their tasks are limited to the handling of a predefined and small number of sterile instruments. Further tasks in the non-sterile area, such as those of a human $\mathrm{CN}$, cannot be fulfilled.

For the implementation of an RCN, as in the AURORA project, a human-machine interface is required that can comprehensively cover the complex communication between the sterile and non-sterile OR environment and which allows for seamless integration of this new technology without causing additional workload.

\section{Methods}

An important first step is to determine the basic requirements for the interface. Through field observations in the OR and user interviews, the communication of the sterile staff with their environment is analyzed. Particular emphasis is placed on the generation of tasks regarding the human $\mathrm{CN}$. Suitable protocols and questionnaires are designed for this purpose.

Based on the information collected, the communication will then be described in detail and thus narrowed down. Through a comprehensive structuring, for example a clustering into different task types, a further, important prerequisite for the feasibility of a human-machine interface will be created.

In order to develop possible communication techniques, literature research and analysis of existing products is carried out.

Based on the developed and structured communication, exemplary use cases and a suitable dialogue design will be modeled.

\section{Results}

In order to realize the goal described above, the provision of a human-machine interface for a non-sterile RCN, the framework condition is defined, that an RCN can only take over a subset of the tasks of a human $\mathrm{CN}$. To enable such a partial integration in a meaningful way, instead of directly communicating with a concrete $\mathrm{RCN}$, an OR-bound, universal interface is developed, which includes any communication of the sterile OR staff with the environment, including the RCN, but also the permanent staff or other devices in the future.

The advantage of an interface, that is permanently present in the OR and is independent of the target instance, is that the work orders of several ORs can be simultaneously collected and prioritized. Furthermore, this concept enables a work order to be subsequently delegated either to a robot or a human, regardless of how the work order is acquired.

The core functionality is defined as the generation of work orders, which are fed by three types of information sources, namely verbal communication, non-verbal communication and context-sensitivity.

Verbal communication includes keywords, freely formulated requests and also further inquiries and suggestions from the system.

Non-verbal communication on the system side includes the visual presentation of images and writing. On the staff side, non-verbal communication includes haptic and gestures interacting with the environment. Concerning the interaction with the environment, its visual perception is required to a certain extent. For example, the instrumenting nurse might present the packaging of a used sterile item and verbally request further items, which requires the precise identification of the packaging.

Specifically with non-verbal communication, one of the most important requirements is to maintain the integrity of the sterile area. Sterile haptic communication and visual presentation of images and writing are made possible, for example, by sterile wireless terminals such as tablets. Another possibility of sterile, non-verbal communication is the clever combination of visual presentation and gestures using modern technologies such as augmented reality and interactive laser projection.

Context-sensitivity requires, on the one hand, the learning of possible surgery situations and their temporal or causal sequence and, on the other hand, the automated recognition of which of these situations is present at a given time. This is done in consideration of previously manually defined framework conditions such as the nature of the surgery and based on comprehensive process models. With help of the knowledge of the present and likely following situation, a more meaningful generation of work orders can take place. A work order can either be generated solely on the basis of the present and predicted situations or from a combination of these situations with explicitly requesting communication on the part of the sterile OR staff.

Such context recognition and prediction was conceptualized and implemented in the research project "IVAP 2025" [2]. From the recognized context, work orders can also be assigned an urgency, which is an important step in prioritizing the handling of multiple work orders. 


\section{Conclusion}

Currently, field observations in the OR are already being conducted. In the analysis of task generation for the $\mathrm{CN}$, particular attention is paid to the sources of information described above. Accordingly, a protocol was designed, which on the one hand records verbally and non-verbally communicated work orders, and on the other hand, also records non-communicated work orders resulting from the situation. Each generated work order is supplemented with further meta-information, such as client, urgency level and phase of the surgery. This is followed by the description and structuring of the collected information as explained above.

For the exploration of possible communication techniques, detailed research of the interaction models of existing linguistic, visual, haptic and gesture-based assistance systems is carried out. These are now widely used in the home and automotive sectors, for example, and can take on numerous tasks.

The presentation will cover first results from literature research and findings from OR field observation data that has been collected and analyzed to date.

\section{References}

[1] Jacob M, Li Y-T, Akingba G, Wachs J (2012): Gestonurse: a robotic surgical nurse for handling surgical instruments in the operating room. Journal of robotic surgery 6 (1): 53-63

[2] Ostler D, Marahrens N, Kohn N, Koller S, Kranzfelder M, Feussner H, Wilhelm D (2018) IVAP 2025-Towards the collaborative operating room. 13th Russian-German Conference on Biomedical Engineering (RGC)

\section{Towards holographic navigation for paediatric nephron sparing wilms tumour surgery: a technical feasibility study}

Q. Eyck ${ }^{1}$, M. Fitski ${ }^{1}$, L. van der Steeg ${ }^{1}$, M. Wijnen ${ }^{1}$

${ }^{1}$ Princess Máxima Centre, pediatric oncology surgery, Utrecht, Netherlands

Keywords Augmented Reality, HoloLens 2, Nephron sparing surgery, Pediatric surgery

\section{Purpose}

A small part of patients suffering from Wilms tumours (WT) can be treated with open nephron sparing surgery (NSS). During this procedure intraoperative ultrasound is used to visualise the exact size of the tumour. Unfortunately, approximately $30 \%$ of the NSS procedures still result in a positive surgical margin. New visualization techniques may help us reduce this percentage.

The size and infiltration of the tumour can be visualized intraoperatively with new techniques such as holographic navigation based on Augmented Reality (AR). The HoloLens 2 projects an overlay of the preoperative imaging in AR onto the open surgical field. Using AR surgical navigation technology, our goal is to reduce positive surgical margins during NSS. Therefore, we have developed an AR application which determines the required overlay with a combination of two algorithms; a registration algorithm based on anatomical landmarks and a tracking algorithm based on QR-code recognition. These self-developed algorithms are validated in this feasibility study. The clinically maximally allowed error for this validation was determined to be five $\mathrm{mm}$ by our surgical team.

Methods

The validation of this self-developed AR application is twofold. We measure the accuracy of the tracking through QR-code recognition

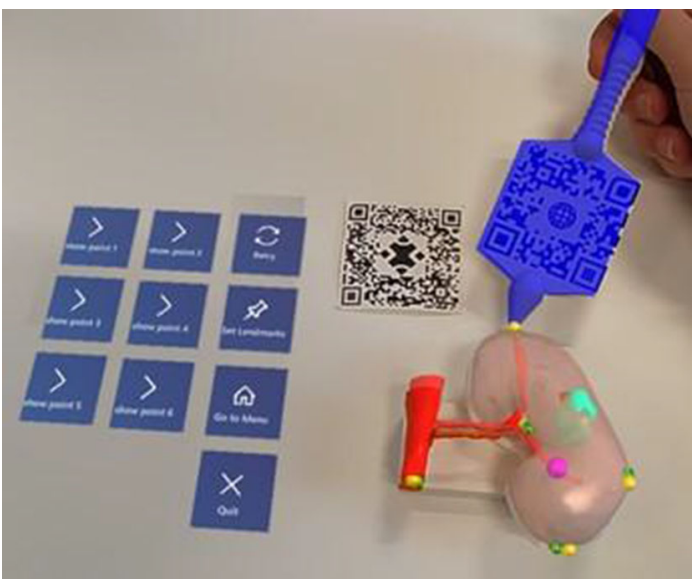

Fig. 1 The holographic overlay of the kidney (salmon), tumour (cyan), artery (red), and a positional marker (purple) on top of the 3D printed model are shown. Additionally, the QR-code used for tracking and the QR-code placed onto the surgical pointer (blue) are visible. The surgical pointer is used to pinpoint the anatomical landmark for the HoloLens 2. There is a slight offset of the overlay due to a difference in height of the camera of the HoloLens 2 and the eyes of the user

and we estimate the accuracy of the resulting registration based on a 3D-printed kidney phantom.

In order to measure the accuracy of the QR-code recognition, a calibration setup was built in which the HoloLens was placed onto a 3D-printed bearer and the QR-code could be moved manually in two different directions. The HoloLens 2 measured the moved distance of the QR-code, which was compared with the actual movement. Additionally, we investigated the minimal workable size of the QRcode without limiting the tracking quality. Three different sizes were used, $5 \times 5 \mathrm{~cm}, 4.5 \times 4.5 \mathrm{~cm}$, and $4 \times 4 \mathrm{~cm}$.

The accuracy of the registration algorithm was derived through a phantom study in which ten volunteers participated. None of our volunteers had prior experience with the AR application. A short introduction on how to use the HoloLens 2 was given and eye-calibration was performed. The volunteers were asked to perform the registration by pinpointing five anatomical landmarks onto the kidney phantom using a surgical pointer, as is shown in figure 1. After mapping of the anatomical landmarks, the holographic 3D model is automatically registered onto the 3D printed phantom. Subsequently, six randomly placed positional markers are highlighted on top of the holographic visualization. The volunteers were asked to mark these holographic positional markers in the real world with a marker. These marked positions were compared with the actual position using a 3Dprinted calibration mold, which allowed us to quantify the amount of misalignment between the holographic 3D model and the corresponding 3D printed model.

Results

We measured the movement of a moving QR-code with three different dimensions $(5 \times 5,4.5 \times 4.5$ and $4 \times 4 \mathrm{~cm})$ in two directions. The mean error and the standard deviation of the movement in the $\mathrm{X}$-axis was $0.29 \mathrm{~mm}(2.2 \mathrm{~mm}), 4.04 \mathrm{~mm}$ (4.6), and $11.54 \mathrm{~mm}$ (3.8) respectively. For the movement in the $\mathrm{Z}$-axis the mean error and standard deviation was $0.47 \mathrm{~mm}$ (3.6), $4.18 \mathrm{~mm}$ (5.6), and 13.57 (8.2) respectively. There was a significant difference in mean error between the $5 \times 5 \mathrm{~cm}$ and $4.5 \times 4.5 \mathrm{~cm}$ QR-codes and the $5 \times 5 \mathrm{~cm}$ and $4 \times 4 \mathrm{~cm} \mathrm{QR-codes}(p<0.001)$.

The accuracy of the registration was measured as a mean distance between the holographic and actual position. This distance varied between volunteers but these differences were statistical not 
Table 1 The mean distance and standard deviation of all 5 marked positions per volunteer

\begin{tabular}{ll}
\hline Volunteer & Mean error $(\mathrm{mm})(\mathrm{SD})$ \\
\hline 1 & $2.80(0.99)$ \\
2 & $2.75(0.92)$ \\
3 & $2.87(1.11)$ \\
4 & $3.01(0.95)$ \\
5 & $2.27(1.60)$ \\
6 & $5.19(4.30)$ \\
7 & $3.93(2.19)$ \\
8 & $2.86(1.00)$ \\
9 & $2.98(1.26)$ \\
10 & $2.95(0.80)$ \\
Mean of all observers & $3.16(1.82)$ \\
\hline
\end{tabular}

significant $(0.056 \leq p \geq 0.963)$. Unfortunately, one positional marker was excluded from the measurements due to a complete misalignment of the holographic marked position and actual position. The mean distance error per volunteer is given in table 1 . Three of the 50 holographic marked positions were placed with a distance greater than $5 \mathrm{~mm}$ of the actual position by two different volunteers. Only one volunteer obtained a mean error above the clinical error of five $\mathrm{mm}$.

\section{Conclusion}

The accuracy obtained by our surgical navigation AR application is within the clinically allowed error and therewith we believe this technique may be feasible for clinical use. The results show that the QR-code tracking capacity of our application is sufficient and that volunteers are capable to apply the anatomical landmark based registration satisfactory. Further implementation of this technique during nephron sparing surgery is recommended. However, intraoperative surgical workload and usability needs to be assessed beforehand. Therefore, we recommend performing a surgical phantom study to further explore this technique in pediatric oncologic surgery.

\section{Image guided decision making for the suspension of patient specific scaphoid replacement}

P. Honigmann ${ }^{1,2,3}$, M. Haefeli ${ }^{2,4}$, J. G. Oonk ${ }^{2}$, G. J. Strijkers ${ }^{2}$, J. G. Dobbe ${ }^{2}$, S. D. Strackee ${ }^{5}$, G. J. Streekstra ${ }^{2,6}$

${ }^{1}$ Kantonsspital Baselland, Hand Surgery, Liestal, Switzerland

${ }^{2}$ Amsterdam UMC, University of Amsterdam, Department of Biomedical Engineering and Physics, Amsterdam Movement Sciences, Amsterdam, Netherlands ${ }^{3}$ Medical Additive Manufacturing, Department of Biomedical Engineering, University Basel, Basel, Switzerland ${ }^{4}$ Kantonsspital Graubünden, Hand Surgery, Chur, Switzerland ${ }^{5}$ Amsterdam UMC, University of Amsterdam, Department of Plastic, Reconstructive and Hand Surgery, Amsterdam Movement Sciences, Amsterdam, Netherlands ${ }^{6}$ Amsterdam UMC, University of Amsterdam, Department of Radiology, Academic Medical Center, University of Amsterdam, Amsterdam Movement Sciences, Amsterdam, Netherlands

Keywords Scaphoid, Patient specific, Prosthesis, Wrist

\section{Purpose}

When aiming for motion preserving procedures in carpal pathologies, patient specific design of carpal bone replacement is one option to maintain individual anatomy. To restore carpal motion and kinematics, a correct fixation of the individualized implant is mandatory. We present an image-based decision-making process to achieve the best suspension.

\section{Methods}

Initial cadaver tests in which we assessed the kinematics of a customized Scaphoid replacement using 4D-CT showed good results in terms of movement and stability of the prosthesis. Based on these data we performed the implantation of a patient specific scaphoid prosthesis in a patient who had a failed reconstruction of a Scaphoid pseudoarthrosis.

Due to a Scaphoidpseudoarthrosis on the contralateral side, the prosthesis was designed according to a patented [1] shape based on a polygon model of the non-united Scaphoid and adapted according to the necessary carpal height needed. The SLS 3D-printed Titanium patient specific prosthesis was implanted using a fibre tape augmented palmaris longus tendon graft fixed to the Trapezium and to the Lunate. Care was taken not to overtighten the suspension to allow for carpal movement. Carpal alignment and stability was checked intraoperatively using fluoroscopy.

Conventional X-rays and 4D-CT scans were taken during follow up visit.

\section{Results}

Intraoperatively, the patient specific prosthesis provided good primary stability of the carpus before carry out the suspension. With the suspension, the implant seemed to be more stable and the carpal alignment stable, especially the scapho-lunate interval. The postoperative clinical course was uneventful and the patient recovered very well from the intervention. Conventional X-rays after 6 and 12 weeks showed a good stability of the prosthesis. To assess carpal kinematics, we performed a $4 \mathrm{D}$-CT scan 12 weeks postoperatively.

The fixation technique of the prosthesis provided sufficient stability during flexion/extension and radial-/ulnar abduction. The carpal alignment was stable but a dorsal intercalated segment instability of the Lunate remained.

\section{Conclusion}

A patient specific design of the Scaphoid replacement is mandatory for good primary stability documented intraoperatively using fluoroscopy. Postoperatively, conventional X-rays were sufficient to check for the position of the prosthesis. For the assessment of carpal alignment and-kinematics and especially decision making to change the suspension, 4D CT imaging was the helpful tool.

When aiming for the best reconstruction of carpal kinematics using a customized prosthesis of the Scaphoid, the correct alignment of the Lunate needs to be considered. We therefore modified the suspension technique using the anatomical front and back ligament reconstruction (ANAFAB) developed by Michael J. Sandow [2] to reconstruct the dorsal intrinsic and palmar extrinsic ligaments according to the findings of the 4D CT scans. Further investigations will show if this is going to improve stability of the lunate as well.

\section{References}

[1] Honigmann P, Schumacher R, Marek R, Büttner F, Thieringer F, Haefeli M (2018) A three-dimensional printed patient-specific scaphoid replacement: a cadaveric study. Journal of Hand Surgery (European Volume), vol. 79, pp. 175319341875763-6, 2018.

[2] Sandow M, Fisher T (2019) Anatomical anterior and posterior reconstruction for scapholunate dissociation: preliminary outcome in ten patients. J Hand Surg Eur Vol. 2020 May;45(4):389-395. https://doi.org/10.1177/17531934198 86536. Epub 2019 Nov 13. PMID: 31718405. 


\section{Augmented reality for improved PSI placement in pelvic tumour resections}

M. García-Sevilla ${ }^{1,2}$, R. Moreta-Martinez ${ }^{1,2}$, D. García-Mato ${ }^{1,2}$, A. Pose-Díez-de-la-Lastra $^{1,2}$, R. Pérez-Mañanes ${ }^{2,3}$, J. Calvo-Haro ${ }^{2,3}$, J. Pascau $^{1,2}$

${ }^{1}$ Universidad Carlos III de Madrid, Departamento de Bioingeniería e Ingeniería Aeroespacial, Leganés, Spain ${ }^{2}$ Instituto de Investigación Sanitaria Gregorio Marañón, Madrid, Spain ${ }^{3}$ Hospital General Universitario Gregorio Marañón, Servicio de Cirugía Ortopédica y Traumatología, Madrid, Spain

Keywords Augmented reality, Pelvic tumour, Patient-specific instruments, 3D-printing

\section{Purpose}

Pelvic tumour resections are challenging due to the bone complexity and the proximity to vital structures. In these surgeries, achieving optimal resection margins is crucial to avoid adverse clinical outcomes and local recurrence. However, the probability of obtaining adequate margins following the conventional approach and under ideal conditions is only $52 \%$ [1].

In recent years, intraoperative navigation has proved to successfully assist in these interventions. The preoperative surgical plan can be easily translated to the operating room, and tumour and anatomical structures can be located accurately thanks to the real-time visual feedback. However, surgical navigation hardware can be expensive and requires experience, a large space in the operating room, and an operator.

Patient-specific instruments (PSIs) have reported similar accuracy to surgical navigation [2]. They are easier to use, more convenient, and faster. They also allow surgeons to focus on the surgical field rather than looking at a screen. However, PSI placement can only be checked subjectively, and therefore correct guidance cannot be verified. As PSIs are customized tools designed to fit in a particular region of the patient's bone, they are highly dependent on the morphology of the bone surface. For instance, the correct placement of a PSI in the iliac crest can become very challenging, as it covers a large area that presents a homogeneous shape. In contrast, the supra-acetabular area is more characteristic, thus less prone to errors.

In this work, we propose augmented reality (AR) guidance to assist in the correct placement of PSIs for pelvic tumour resections. Specifically, we focus on type I resections, where two PSIs are placed, one in the supra-acetabular region and one in the iliac crest. Using a reference marker placed on the supra-acetabular PSI, we can display the bone and iliac crest PSI models in their correct position. These models are then used as guidance for placement.

\section{Methods}

We conducted an experiment with a bone phantom and a total of 12 PSIs ( 6 for the supra-acetabular region and 6 for the iliac crest). The pelvic bone used for the phantom was segmented from the CT of a patient using 3D Slicer platform. The design of the PSIs and the phantom was carried out in Meshmixer software (Autodesk, Inc., USA). The PSIs were designed in different locations and with varying shapes inside their target area. Finally, we printed all the models in PLA using the Ultimaker 3 Extended (Ultimaker B.V., Netherlands) desktop 3D printer.

We created two versions of the phantom, a normal and a realistic one. The first one was simply the printed bone, whereas the realistic one included a silicone layer unequally distributed across the surface. This layer simulated the tissue present in real scenarios, where the bone surface is not entirely exposed.

A smartphone AR application was developed for guiding PSI placement (Figure 1) using Unity platform and Vuforia development kit. The application uses the internal camera to detect a cubic marker (3D-printed) presenting a known black-and-white pattern. This

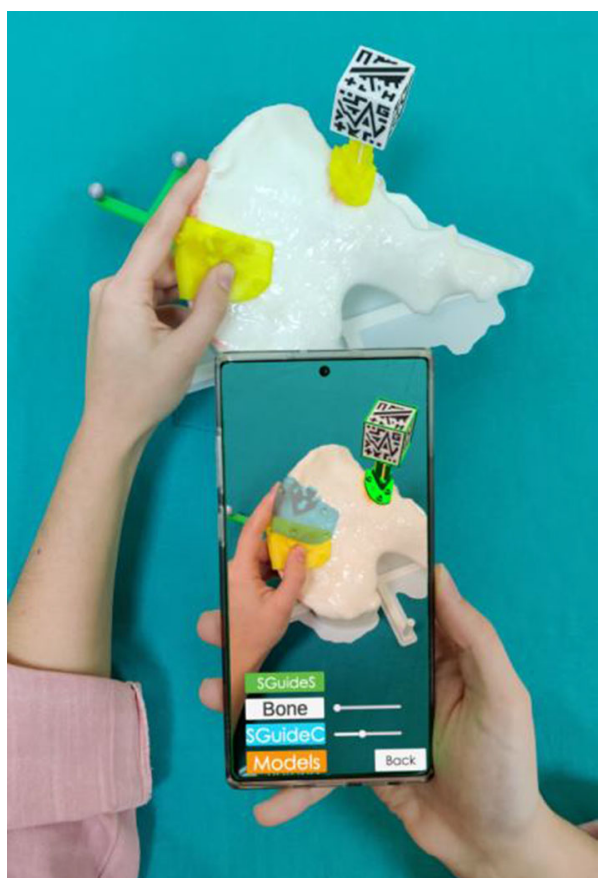

Fig. 1 PSI placement in the phantom using the AR app for guidance

marker is placed on the supra-acetabular PSI through a socket. The application detects the marker and, based on the location of the supraacetabular PSI in the virtual plan, displays the relative position of the bone (used to verify the placement of the supra-acetabular PSI) and the PSIs overlaid on the simulated surgical field.

In order to record the placement of the PSIs in the bone and compare it with the virtual plan, we used the Polaris Spectra (NDI, Waterloo, Canada) optical tracking system. A dynamic reference frame was attached to the phantom and registration was performed using pinholes included in the phantom design. Each PSI also contained 4 pinholes to record their position using the optical tracker.

A total of 3 users placed the 6 pairs of PSIs, both manually and using AR. The process was repeated twice, one for each version of the phantom. We recorded the position of the 4 pinholes in each PSI after their placement and measured the time required for each method. The mean distance between the recorded points in every PSI and their position in the virtual plan was computed for posterior analysis.

\section{Results}

Table 1 shows the mean, standard deviation, and maximum distances of the PSIs to their virtual plan for each method and each phantom type. The results obtained when using AR for guidance present lower mean values (below $2 \mathrm{~mm}$ ) than freehand placement in both phantoms. The variability is also reduced, especially for the iliac crest PSIs, where maximum errors of almost $1 \mathrm{~cm}$ are reduced to $2.37 \mathrm{~mm}$. The realistic phantom presents higher errors in all cases. The use of AR for placement increased the total time in approximately $1 \mathrm{~min}$.

\section{Conclusion}

The results obtained prove the benefits of using AR for PSI placement. The time added to the procedure is negligible while providing precision and avoiding high errors. The results also highlight the importance of using realistic phantoms to better resemble real scenarios. It is easy to find the correct placement with a smooth bone surface (from which PSIs are designed) but this is usually not the case in real interventions.

To conclude, AR is an effective tool to improve PSI placement. Further studies should be conducted to ensure its feasibility and accuracy inside the OR. 
Table 1 Distances of the recorded points in every PSI (iliac crest and supra-acetabular) to their planned position for each placement method (freehand or AR) and each phantom (normal or realistic)

\begin{tabular}{llllll}
\hline & \multicolumn{2}{l}{ Normal Phantom } & & \multicolumn{2}{l}{ Realistic Phantom } \\
& Freehand & AR & & Freehand & AR \\
\hline Iliac crest & & & & \\
Mean & 2.4 & 1.17 & & 3.26 & 1.52 \\
Std & 1.67 & 0.42 & & 1.88 & 0.39 \\
Max & 6.18 & 2.18 & & 9.48 & 2.37 \\
\multicolumn{2}{l}{ Supra-acetabular } & & & \\
Mean & 1.07 & 0.92 & & 2.14 & 1.66 \\
Std & 0.36 & 0.25 & & 0.88 & 0.53 \\
Max & 1.8 & 1.44 & & 4.56 & 2.61 \\
\hline
\end{tabular}

\section{Acknowledgements}

Supported by projects PI18/01625, and IND2018/TIC-9753.

\section{References}

[1] Cartiaux O, Docquier PL, Paul L, Francq BG, Cornu OH, Delloye C, Raucent B, Dehez B, Banse X (2008) Surgical inaccuracy of tumor resection and reconstruction within the pelvis: an experimental study. Acta Orthop 79:695-702. https://doi.org/10.1080/17453670810016731

[2] Wong KC, Sze KY, Wong IOL, Wong CM, Kumta SM (2016) Patient-specific instrument can achieve same accuracy with less resection time than navigation assistance in periacetabular pelvic tumor surgery: a cadaveric study. Int J Comput Assist Radiol Surg 11:307-316. https://doi.org/10.1007/ s11548-015-1250-x

\section{Identification of implant type from total knee arthroplasty images based on machine learning}

\section{Kishino ${ }^{1}$, T. Yamazaki ${ }^{1}$, F. Itami ${ }^{1}$, T. Tomita ${ }^{2}$, K. Sugamoto ${ }^{1}$ Saitama Institute of Technology, Information Systems, Fukaya, Saitama, Japan ${ }^{2}$ Osaka University Graduate School of Medicine, Orthopaedic Biomaterial Science, Suita, Osaka, Japan}

Keywords discrimination performance, total knee arthroplasty images, machine learning, convolutional neural network

\section{Purpose}

Accurate assessment of 3D kinematics after total knee arthroplasty (TKA) is very important for understanding the complexity of knee joint mechanics after surgery and for evaluating the outcome of surgical techniques. To achieve 3D kinematic analysis of TKA, 2D/ 3D registration techniques, which use $\mathrm{X}$-ray fluoroscopic images and computer aided design (CAD) model of the knee implants, have been applied to clinical cases. In most conventional techniques, although the accuracy of 3D TKA kinematic analysis for clinical application has been achieved, the analysis or measurement process requires some manual operations and is still labor-intensive and time-consuming work.

For such a serious problem of manual operations for clinical application, we have developed some elemental techniques [1] for full automation of 3D TKA kinematic measurement based on 2D/3D registration using a single-plane fluoroscopic image and CAD model of the knee implant. As one of them, to automatically identify the type of knee implant from X-ray fluoroscopic image is important. Such an automatic identification of implant type is thought to be also useful for supporting TKA diagnosis using simple X-ray radiograph.

In this study, therefore, we conduct a basic investigation of discrimination performance of implant type based on machine learning, using many kinds of TKA silhouette images. Specifically, we examine the identification effect by Mahalanobis distance in conventional machine learning, and also the discrimination performance by convolutional neural network $(\mathrm{CNN})$ and its visualization by gradientweighted class activation mapping (Grad-CAM) [2].

\section{Methods}

To identify the implant type from TKA silhouette images, in this study, discrimination method by Mahalanobis distance in conventional machine learning (pattern recognition) and discrimination one by $\mathrm{CNN}$ (deep learning) were used respectively.

In order to validate the discrimination performance of implant type using each method, two experiments using synthetic images (computer simulation test) and real X-ray images were performed.

In the first experiment using synthetic images, four type of knee implant CAD model (F, N, P and V type) were used, and a set of 441 synthetic silhouette images were created for each implant type in known typical orientations using perspective projection model. Therefore, a total of $1764(4 \times 441)$ synthetic silhouette images were created for femoral and tibial component, respectively. For identification of implant type from synthetic images using conventional machine learning (pattern recognition), two features which are effective for identification (in this study, contour complexity, and area ratio of implant silhouette relative to bounding box image) were utilized. In order to verify the identification effect by Mahalanobis distance which considers the distribution of the data, the identification using Euclidean distance was also performed. For identification of implant type from synthetic images using $\mathrm{CNN}$, a simple network which has one convolution (and pooling) layer and two features map $(3 \times 3$ filter size), AlexNet and VGG16 were used respectively. To ensure the validity for each method, fivefold cross validation was applied using 1764 synthetic silhouette images.

In the second experiment using real X-ray images, three type of knee implant silhouette images (number of images for A, B and C type are 325,325 , and 301 images) were collected, and a total of 951 real X-ray images were used. As a discrimination method to identify the implant type from actual $\mathrm{X}$-ray images, $\mathrm{CNN}$, that is, a simple network which has one convolution (and pooling) layer and two features map $(3 \times 3$ filter size), AlexNet and VGG16 were used respectively. To ensure the validity for each $\mathrm{CNN}$ method, tenfold cross validation was applied using 951 real X-ray images.

Results

The results of the experiment for synthetic images using conventional machine learning are summarized in Table 1 . The number of misidentified images using the Mahalanobis distance compared to the Euclidean distance was reduced for each implant type, and the identification rate (discrimination performance) was improved from $71.2 \%$ to $83.7 \%$ for femoral component and from $33.0 \%$ to $71.9 \%$ for tibial component. As results of the experiment for synthetic images using $\mathrm{CNN}$, there were no misidentified images in all networks (a simple network, AlexNet and VGG16), and the identification rate (discrimination performance) was $100 \%$ for femoral and tibial component. Fig. 1 shows representative four type of knee implant silhouette images (F, N, P and V type) and example for the results of Grad-CAM visualization identified using VGG16.

As results of the experiment for real X-ray images using $\mathrm{CNN}$, identification rates using a simple network, AlexNet and VGG16 were $94.5 \%, 99.7 \%$ and $99.6 \%$ for femoral component, and $95.8 \%, 100 \%$ and $99.9 \%$ for tibial component, respectively. In addition, the results 
Table 1 Number of misidentified images for each implant type and identification rate for synthetic images using conventional machine learning

\begin{tabular}{|c|c|c|c|c|c|c|}
\hline \multirow[t]{2}{*}{$\begin{array}{l}\text { Types of } \\
\text { components }\end{array}$} & \multirow[t]{2}{*}{ Distance type } & \multicolumn{4}{|c|}{$\begin{array}{l}\text { Number of misidenti- } \\
\text { fied images }\end{array}$} & \multirow[t]{2}{*}{$\begin{array}{l}\text { Identification } \\
\text { rate }(\%)\end{array}$} \\
\hline & & $\begin{array}{l}\text { F- } \\
\text { type }\end{array}$ & $\begin{array}{l}\mathrm{N}- \\
\text { type }\end{array}$ & $\begin{array}{l}\mathrm{P}- \\
\text { type }\end{array}$ & $\begin{array}{l}\text { V- } \\
\text { type }\end{array}$ & \\
\hline \multirow[t]{2}{*}{$\begin{array}{l}\text { Femoral } \\
\text { component }\end{array}$} & $\begin{array}{l}\text { Euclidean } \\
\text { distance }\end{array}$ & 74 & 148 & 202 & 84 & 71.2 \\
\hline & $\begin{array}{l}\text { Mahalanobis } \\
\text { distance }\end{array}$ & 30 & 126 & 123 & 9 & 83.7 \\
\hline \multirow[t]{2}{*}{$\begin{array}{l}\text { Tibial } \\
\text { component }\end{array}$} & $\begin{array}{r}\text { Euclidean } \\
\text { distance }\end{array}$ & 184 & 225 & 376 & 396 & 33.0 \\
\hline & $\begin{array}{l}\text { Mahalanobis } \\
\text { distance }\end{array}$ & 123 & 122 & 105 & 147 & 71.9 \\
\hline
\end{tabular}
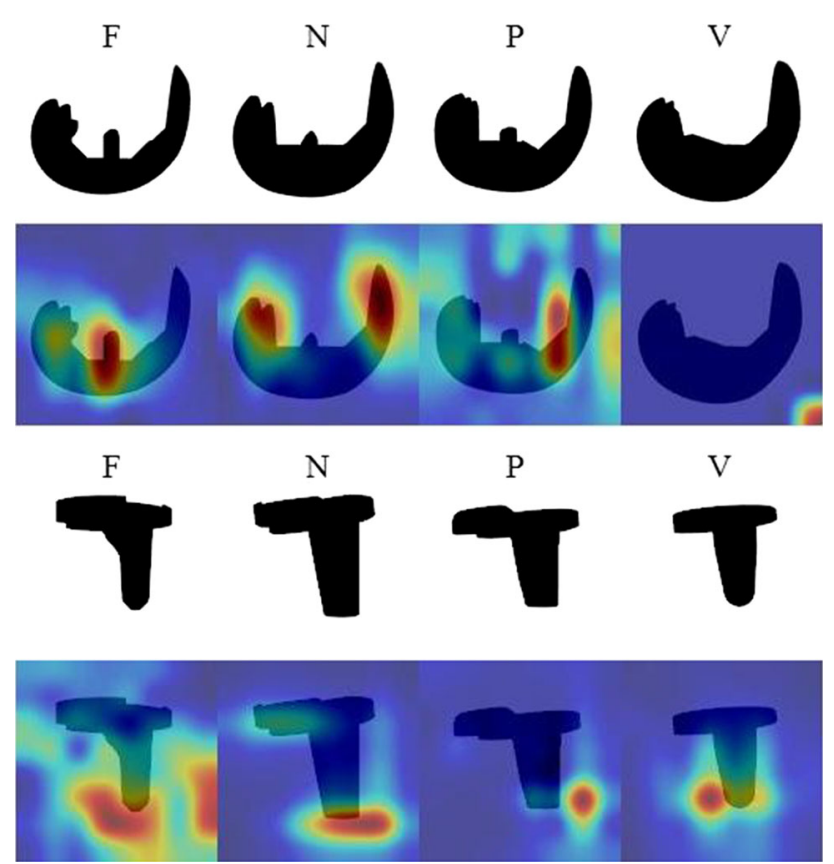

Fig. 1 Representative four type of knee implant silhouette images (F, $\mathrm{N}, \mathrm{P}$ and $\mathrm{V}$ type) and example for the results of Grad-CAM visualization identified using VGG16

of Grad-CAM visualization identified using each network showed a reasonable and explainable heatmap pattern.

\section{Conclusion}

In this study, to automatically identify the implant type from TKA silhouette images, a basic investigation of discrimination performance of implant type based on machine learning was conducted.

In the result of the experiment for synthetic images, the identification effect by Mahalanobis distance in conventional machine learning was confirmed as shown in Table 1. The reason for this identification effect is thought that the distribution of two features used in this study is close to a Gaussian distribution. While, as results of the experiment for synthetic images using $\mathrm{CNN}$, there were no misidentified images in all networks, and the identification rate (discrimination performance) was $100 \%$.

In the result of the experiment for real X-ray images using $\mathrm{CNN}$, for all networks used in this study, the identification rate was very high. In particular, identification rates using AlexNet and VGG16 were close to $100 \%$, and this suggests that automatic identification of the implant type from actual X-ray images is possible.

\section{References}

[1] Yamazaki T, Tomita T, Sato Y, Yoshikawa H, Sugamoto K (2019) Robust 3D kinematic measurement of femoral component using machine learning. Proc. IJCARS 14(1):S156-157

[2] Selvaraju R.R, Cogswell M, Das A, Vedantam R, Parikh D, Batra D (2020) Grad-CAM: visual explanations from deep networks via gradient-based Localization. IJCV 128:336-359

\section{$\overline{\text { Blood vessel regions segmentation from laparoscopic }}$ videos using fully convolutional networks with multi field of view input}

S. Morimitsu ${ }^{1}$, S. Yamamoto ${ }^{1}$, T. Ozawa ${ }^{1}$, T. Kitasaka ${ }^{2}$,

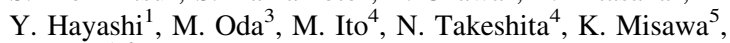
K. Mori ${ }^{1,6,7}$

${ }^{1}$ Nagoya University, Graduate School of Informatics, Nagoya, Japan

${ }^{2}$ Aichi Institute of Technology, School of Information Science,

Toyota, Japan ${ }^{3}$ Nagoya University, Information and Communications, Nagoya, Japan ${ }^{4}$ National Cancer Center Hospital East, Chiba, Japan ${ }^{5}$ Aichi Cancer Center Hospital, Nagoya, Japan ${ }^{6}$ Nagoya University, Information Technology Center, Nagoya, Japan ${ }^{7}$ Research Center for Medical Bigdata, National Institute of Informatics, Tokyo, Japan

Keywords Blood vessel segmentation, Multi field of view, U-Net, Laparoscopic video

\section{Purpose}

Laparoscopic surgery is widely performed as minimally invasive surgery. This surgery is generally difficult compared with the conventional open surgery. Therefore, surgical assistance systems for laparoscopic surgery have been studied. In these systems, scene recognition from laparoscopic videos is important to generate surgical assistance information. There are many research on laparoscopic scene analysis such as surgical instruments and anatomical structures segmentation. Our research group conducted segmentation of the inferior mesenteric artery (IMA) regions, which are important anatomical structures in laparoscopic colorectal surgery, from laparoscopic videos using U-Net [1] and convLSTM U-Net [2]. In this method, we introduced long short-term memory (LSTM) to U-Net for improving segmentation accuracy along time axis. The results showed that the convLSTM U-Net enabled stable segmentation along the time axis. However, this method was sensitive to changes of blood vessel size in the images. The segmentation accuracy was affected by change of distance between the laparoscopic camera and the blood vessels due to the camera movement in the same surgical scene. In this paper, to reduce this problem, we introduce a multi field of view (FOV) framework to fully convolutional networks (FCNs) for segmentation.

\section{Methods}

In the proposed method, we input multiple images having different FOV sizes to the FCN. Multi FOV images are generated by clopping and resizing the original laparoscopic images. This operation simulates distance changes between the laparoscopic camera and the blood vessels. Images contains variations of blood vessel sizes are generated. We input three different FOV images to FCN. Size of these three 
images are $512 \times 512$ pixels, $256 \times 256$ pixels, and $128 \times 128$ pixels. We utilize U-Net with dilated convolution as the FCN. U-Net has encoder and decoder parts. We add a dilated convolution layer before the decoder part. Furthermore, we extend this FCN to handle multi FOV inputs. Our multi-input U-Net has three encoder parts to process three FOV input images, respectively. Feature maps obtained from these encoders are concatenated and fed to the dilated convolution layer. Skip connections are made between the encoder part corresponding to the largest image input and decoder part.

We train the proposed network using laparoscopic images and manually segmented IMA regions for training. We use Dice loss as loss function and Adam as optimizer in the training. Data augmentation using rotation, scaling, and Gaussian blur is used to increase the training data. In the inference using the trained network, we input laparoscopic images for inference and obtain segmentation results.

\section{Results}

We extracted the IMA from laparoscopic videos using the proposed method. Dataset was created by selecting frames in which IMA is appeared from 37 laparoscopic videos of laparoscopic sigmoid colectomy and laparoscopic high anterior resection for rectal cancer. Total number of frames in the dataset was 2566 frames. For evaluation, we performed fivefold cross validation. Mean Dice coefficients of extraction results by U-Net [1], convLSTM U-Net [2], and the proposed multi input U-Net were $0.386,0.476$ and 0.426 , respectively. An example of extraction results is shown in Fig. 1.

The blue regions in this figure indicate the extracted IMA regions. These results showed that the proposed method could extract the IMA regions from laparoscopic videos. The proposed method correctly segmented IMA regions even if the distance between the laparoscopic camera and IMA region changes, as shown in Fig. 1. We consider that the proposed method was robust to size changes of the blood vessels in the scene by using multi FOV input. However, mean Dice coefficient was reduced compared with the previous method [2]. Since each encoder provides different feature maps, imposing different weights to encoders will improve segmentation accuracy.

Conclusion

In this paper, we reported the blood vessel segmentation method using multi field of view input from laparoscopic videos. The experimental results showed that the proposed method could extract IMA regions from laparoscopic video. Especially, the proposed method correctly segmented IMA regions even if the distance between the laparoscopic camera and IMA region changes. Future work includes improvement of segmentation accuracy using weighing of the feature maps obtained from each encoder.

\section{Acknowledgements}

Parts of this research were supported by the AMED Grant Numbers 18he1802002h402, 19he2102001h0701, 20he2102001h0702, JP19lk1010036, JP20lk1010036 and the MEXT/JSPS KAKENHI Grant Numbers 17H00867, 17K20099, 26108006

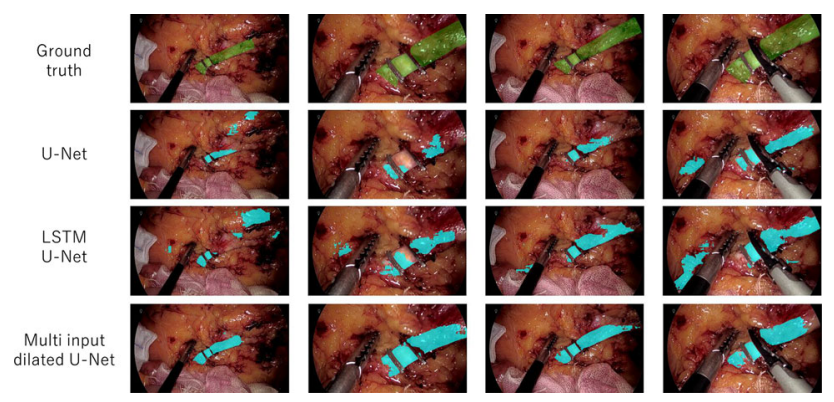

Fig. 1 An example of extraction results by U-Net, convLSTM U-Net, and proposed multi input U-Net

\section{References}

[1] Morimitsu S, Itoh H, Ozawa T, Oda H, Kitasaka T, Sugino T, Hayashi Y, Takeshita N, Ito M, Oda M, Mori K (2019) Automatic segmentation of attention-aware artery region in laparoscopic colorectal surgery. Int J CARS 14(Sup.1):s41-42

[2] Morimitsu S, Yamamoto S, Ozawa T, Kitasaka T, Hayashi Y, Oda M, Ito M, Takeshita N, Misawa K, Mori K (2020) Blood vessel segmentation from laparoscopic video using ConvLSTM U-Net. Int J CARS 15(Sup.1): S63-S64

\section{Soft tissue needle punctures-a study using acoustic emission and force}

A. Illanes ${ }^{1}$, M. Sabieleish ${ }^{1}$, K. Heryan ${ }^{2}$, A. Boese ${ }^{1}$, M. Friebe ${ }^{1}$

${ }^{1}$ Otto-von-Guericke Universität, Medical Faculty, INKA-Innovation Laboratory for Image Guided Therapy, Magdeburg, Germany ${ }^{2} \mathrm{AGH}$ University of Science and Technology, Department of Measurement and Electronics, Kraków, Poland

Keywords needle puncture, audio guidance, force feedback, needle interventions

\section{Purpose}

Percutaneous needle insertion is one of the most common minimally invasive procedures. Needle punctures are required in several applications such as laparoscopic access, biopsies, or regional anesthesia. The clinician's experience and accompanying medical imaging are essential to complete these procedures safely. Imaging, however, may come with inaccuracies due to artifacts, mainly generated by the needle device.

Sensor-based solutions have been proposed to improve accuracy by acquiring additional guidance information from the needle itself or the needle path. This typically requires sensors to be embedded in the needle tip, leading to direct sensor-tissue contact, associated sterilization issues, and added complexity and cost.

A novel concept for acquiring additional complementary information for guiding minimally invasive instruments was previously suggested and presented by our group [1], using an audio sensor connected to the proximal end of a tool to capture interactions between the tip the tissue. The obtained signal can then be processed to extract useful guidance information that can then be mapped to provide feedback to surgeons during minimally invasive procedures.

We were able to show promising results for monitoring medical interventional devices such as needles and guide wires. It was also demonstrated that audio could contain valuable information for monitoring tip/tissue interaction by studying the relationship between force and audio during needle punctures [2]. This study has been extended in this presented work to show that the obtained relationship between force and audio does not depend on the needle insertion velocity. Significant signal-to-signal correlations are obtained between audio and force during a puncture event occurring at four different velocities.

\section{Methods}

For the signal analysis, the previous dataset [1] was used, where audio signals were recorded using a stethoscope connected to a microphone attached to the proximal end of a needle via a $3 \mathrm{D}$ printed adapter. This dataset consists of 80 audio recordings acquired during automatic insertion of an 18G $200 \mathrm{~mm}$ length biopsy needle (ITP, Germany) into an ex vivo porcine tissue phantom. The insertion was performed automatically using a testing machine (Zwicki, Zwick GmbH \& Co.KG, Ulm) at an insertion velocity of $3 \mathrm{~mm} / \mathrm{s}$ that recorded the axial needle insertion force. The audio and force frequencies sampling was $44100 \mathrm{~Hz}$ and $100 \mathrm{~Hz}$, respectively. The acquisition of force and audio was synchronized using a trigger event visible in both signals. 
Using the same setup, experiments at three other velocities, 6, 10, and $14 \mathrm{~mm} / \mathrm{s}$, were also performed. Thirty new audio and force recordings were generated per velocity.

For relating force and audio signal dynamics, indicators from both signals are first extracted. The audio signal is processed using a bandpass filter followed by homomorphic envelope extraction. An indicator for enhancing the force signal's curvature information was extracted using a 2nd-degree polynomial fitting inside a sliding window [2] and then by computing the homomorphic envelope.

Finally, a signal-to-signal correlation by computing the Pearson coefficient is performed between the audio and force indicators.

\section{Results}

Table 1 shows the average and standard deviation of the Pearson coefficients obtained from the four tested velocities' audio and force indicators. It is possible to observe that the average Pearson coefficient for all the velocities is predominantly over 0.5 , arriving to 0.71 and 0.72 for the extreme lower and higher velocities.

Fig. 1 displays a further analysis concerning the obtained correlations, where the accumulative histograms of the Pearson coefficients of insertions at each velocity are displayed. This analysis confirms the results shown in Table 1. For all the insertion velocities, more than $80 \%$ of the recordings show Pearson coefficients between the audio and force indicators over 0.5 . Mainly for $3 \mathrm{~mm} / \mathrm{s}$ and $14 \mathrm{~mm} / \mathrm{s}$ insertion velocities, more than $85 \%$ of the correlations are even over 0.6. These correlations are high, considering the completely different nature between audio and force sensors.

Fig. 2 shows examples of high correlations between the audio and force indicators for the $6 \mathrm{~mm} / \mathrm{s}$ and $14 \mathrm{~mm} / \mathrm{s}$ velocities. For both recordings, it is possible to visualize the entry and exit of the porcine tissue clearly. It is also possible to see how the audio indicator's main dynamics can follow the dynamics obtained from the force indicator. Puncture of important tissues (mainly fascia) resulting in a high peak

Table 1 Average and standard deviation of the Pearson coefficients for each tested velocity

\begin{tabular}{llll}
\hline $3 \mathrm{~mm} / \mathrm{s}$ & $6 \mathrm{~mm} / \mathrm{s}$ & $10 \mathrm{~mm} / \mathrm{s}$ & $14 \mathrm{~mm} / \mathrm{s}$ \\
\hline $0.72 \pm 0.10$ & $0.63 \pm 0.09$ & $0.61 \pm 0.15$ & $0.71 \pm 0.14$ \\
\hline
\end{tabular}

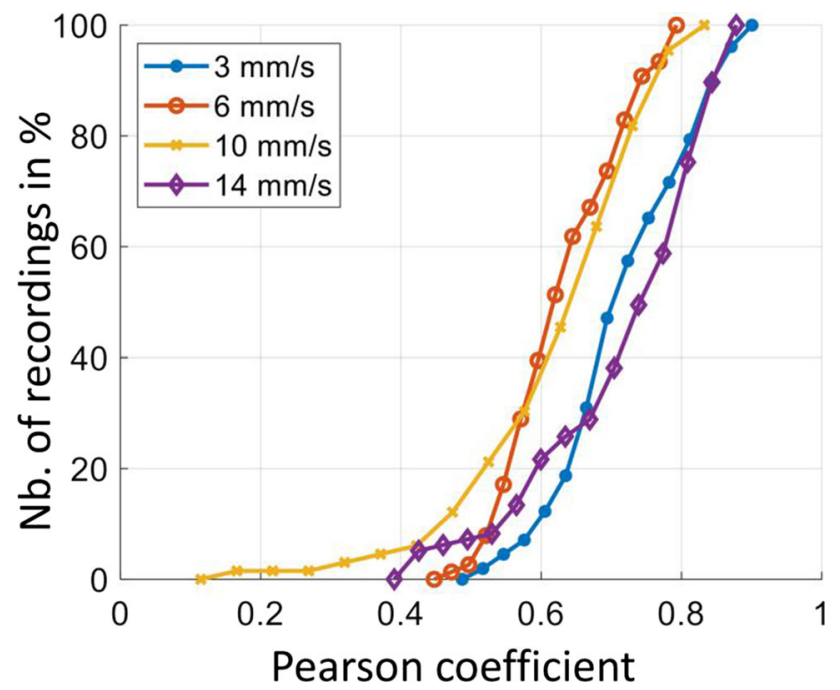

Fig. 1 Cumulative histograms of the Pearson coefficients obtained between the audio and force indicators for the four tested insertion velocities
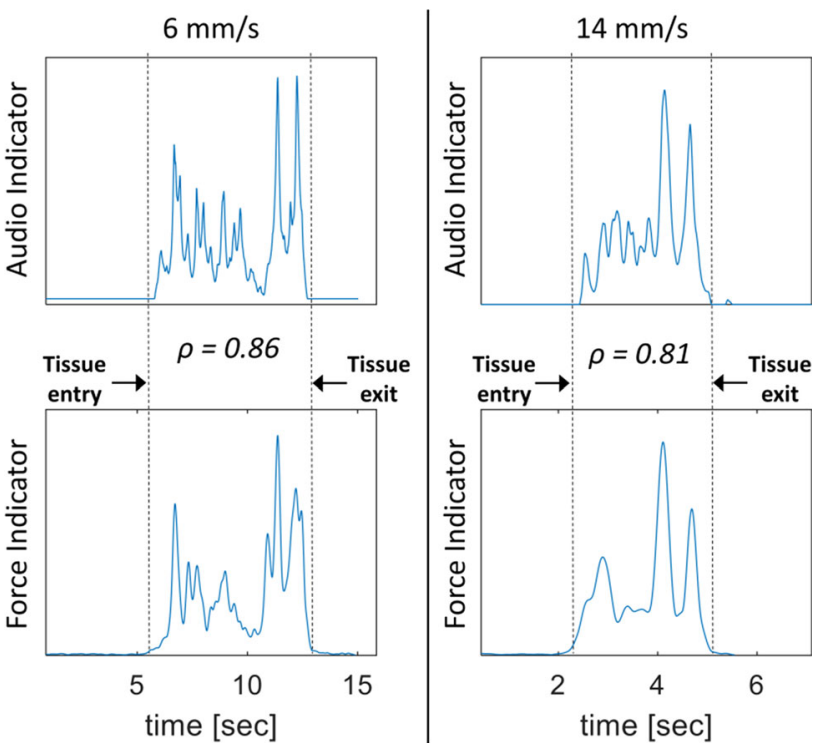

Fig. 2 Examples of force and audio indicators where high correlations were obtained

in the force generates significant audio excitations that are enhanced and delineated thanks to the homomorphic envelope indicator. All these results suggest that the higher is the curvature in the force signal, the higher is the resulting audio excitation in terms of cumulative events enveloped by the audio indicator.

\section{Conclusion}

In this work, we explored the audio dynamics generated from the tip/ tissue interaction during needle insertion into soft tissue using audio and force sensors. Different needle insertion velocities were tested to analyze the signal dynamical responses in both sensors.

The results confirm what has been presented in previous works. Audio contains valuable information for monitoring needle tip/tissue interaction dynamics. Significant dynamics that can be obtained from a well-known sensor as force can also be extracted from audio. The results also show that the audio guidance approach is robust to insertion velocities in terms of puncture acoustic excitations.

\section{References}

[1] Illanes A, Boese A, Maldonado I, Pashazadeh A, Schaufler A, Navab N, Friebe M (2018) Novel clinical device tracking and tissue event characterization using proximally placed audio signal acquisition and processing. Scientific reports, 8(1), pp. $1-11$

[2] Illanes A, Boese A, Friebe M, Hansen C (2020) Feasibility Check: Can Audio Be a Simple Alternative to Force-Based Feedback for Needle Guidance? In International Conference on Medical Image Computing and Computer-Assisted Intervention (pp. 24-33). Springer, Cham.

\section{Comparison of different spectral cameras for image- guided surgery}

E. Wisotzky ${ }^{1,2}$, B. Kossack ${ }^{1}$, R. Mühle ${ }^{3}$, W. Markgraf ${ }^{3}$, A. Hilsmann $^{1}$, P. Eisert ${ }^{1,2}$

${ }^{1}$ Fraunhofer Heinrich-Hertz-Institute, Vision and Imaging Technologies, Berlin, Germany ${ }^{2}$ Humboldt-Universität zu Berlin, 
Institut für Informatik, Berlin, Germany ${ }^{3} \mathrm{TU}$ Dresden, Dresden, Germany

Keywords Image-guided surgery, Hyperspectral imaging, Multispectral image processing, Kidney analysis

\section{Purpose}

One of the major problems for the transplantation medicine is the lack of donor grafts as the need for organs far exceeds the number of available donor organs. This high demand for donor organs shows in particular the need to increase the number of successfully transplanted organs ensuring an optimal use of the few donor organs is urgently required. A contactless optical evaluation tool to monitor organ quality before transplantation would therefore be of great interest. Multispectral and hyperspectral imaging (MHSI) in medical applications can provide information about the physiology, morphology and composition of tissues and organs. The use of these technologies enables the evaluation of biological objects and can be potentially applied as an objective assessment tool for medical professionals. For example, in organ preservation prior to transplantation, HMSI could be used to continuously monitor functional parameters of the organ non-invasively. Based on the evaluation of organ quality, surgeons could substantiate their decision-making process in general as well as, in particular, it could help to ensure the appropriate use of donor organs and increase the number of successful transplantations.

\section{Methods}

In this study, four MHSI systems (one hyperspectral pushbroom camera and three multispectral snapshot cameras) were examined for their applicability of detecting specific tissue properties to ease the decision-making process during surgery especially during organ transplantations. The four cameras were used in three different setups: First, the pushbroom camera setup captured the complete spectral data set of the first spatial dimension by recording a slit in one shot. The second spatial component was created by moving the slit. Second, two snapshot cameras $(4 \times 4$-VIS and $5 \times 5$-NIR) were combined to a multispectral 41-bands setup. Both cameras followed the same capturing principle but covered different spectral intervals $(4 \times 4$ VIS: $463 \mathrm{~nm}$ to $638 \mathrm{~nm}$ and $5 \times 5$-NIR: $693 \mathrm{~nm}$ to $966 \mathrm{~nm}$ ). The third snapshot camera $(3 \times 3$-VIS $)$ covered the same spectral interval as the $4 \times 4$-VIS camera but having fewer bands. This allowed an analysis of the minimum required number of spectral bands (16 bands of $4 \times 4$-VIS vs. 8 bands of $3 \times 3$-VIS) needed for robust organ surveillance. All setups with illumination setting are described in detail in [1] (Table 1).

A spectrometer was used as a reference system, beforehand calibrated with a standardized color chart. The spectral accuracy of the cameras reproducing chemical properties of different biological objects (porcine blood, four different physiological and pathological porcine tissues-kidney, lung, heart and brain) was analyzed using the Pearson correlation coefficient. To underline the applicability of MHSI driven analysis of tissue characteristics during kidney

Table 1 Pearson coefficient between the averaged spectral curves of the different acquisition methods and the acquired spectrometer ground truth data

\begin{tabular}{llllll}
\hline Camera Setup & Kidney & Lung & Heart & Brain & $\lambda$-area $(\mathrm{nm})$ \\
\hline 1. Pushbroom & 0.9938 & 0.9978 & 0.9957 & 0.9982 & $500-995$ \\
2. 41-bands & 0.9707 & 0.9822 & 0.9817 & 0.982 & $460-970$ \\
3. 3 × 3-VIS & 0.8369 & 0.8397 & 0.5894 & 0.8533 & $400-700$
\end{tabular}

The curves of the pushbroom, the 41-bands and the $3 \times 3$-VIS camera setup are linearly fitted between the single bands in the given wavelengths area

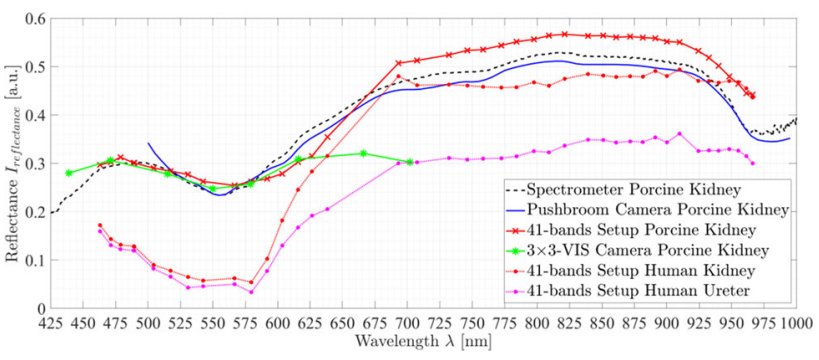

Fig. 1 Different kidney measurements of healthy porcine kidney tissue using all four setups (spectrometer ground truth, pushbroom, 41-bands and $3 \times 3$-VIS) as well as in vivo human kidney and ureter tissue during transplantation using the 41-bands setup

transplantation, the 41-bands snapshot setup has been used to acquire spectral data of in vivo human kidney and ureter.

To obtain the reflectance spectrum from the measured raw data, each image was corrected according to

$\mathrm{I}_{\text {reflectance }}=\left(\mathrm{I}_{\text {raw }}-\mathrm{I}_{\text {dark }}\right) /\left(\mathrm{I}_{\text {white }}-\mathrm{I}_{\text {dark }}\right)$

where $I_{\text {reflectance }}$ is the resulting reflectance spectrum, $I_{\text {raw }}$ is the measured raw data, $I_{\text {dark }}$ contains the dark reference data and $I_{\text {white }}$ is the white reference intensity spectrum. The entire calibration process is described in detail in [2].

\section{Results}

All four examined MHSI cameras are able to provide the characteristic spectral properties of the porcine blood and tissue samples. The pushbroom camera setup and the multispectral 41-bands setup achieves Pearson coefficients of at least 0.97 compared to the ground truth spectrometer data, indicating a very high positive correlation. Only the $3 \times 3$-VIS snapshot camera setup performs moderate to high positive correlation ( 0.59 to 0.85 ). The correlation coefficients of the three setups to the spectrometer ground truth data of the four porcine tissue samples are presented in Tab. 1.

All three setups allow exact reproduction of the physiological conditions of the analyzed anatomical porcine structures, see Fig. 1. The precision of these representations is dependent of each setup, meaning the choice of the optimal setup would be dependent on the specific clinical aim. Different tissue characteristics like oxygen saturation $(\lambda=400-575 \mathrm{~nm})$, water $(\lambda=970 \mathrm{~nm})$ or hemoglobin $(\lambda=760 \mathrm{~nm})$ concentration can be derived using all setups, whereas a higher number of spectral bands is preferable for a snapshot setup $(4 \times 4$-VIS vs. $3 \times 3$-VIS camera). Thus, both basic acquisition principles (pushbroom and snapshot) can be feasible for clinical tissue analysis and differentiation or organ surveillance. Physiological conditions can be analyzed, helping the surgeon to evaluate organ quality as well as to detect dangerous incidents, like bad organ perfusion, during re-perfusion of the organ.

First measurements with the 41-bands snapshot setup during kidney transplantation underline the achieved porcine analyses. The analyzed kidney and ureter samples differ clearly between each other as well as from the porcine kidney, see Fig. 1 .

\section{Conclusion}

We have analyzed the possible use of three different spectral acquisition setups for intraoperative organ surveillance. All camera setups are able to reconstruct the spectral behavior of the analyzed organs in their available wavelength range. For accurate and robust analysis of clinically relevant biological materials, fine scanning of the analyzed spectral range is essential. The knowledge of the suitability of MHSI camera for accurate measurement of chemical properties of biological objects offers a good opportunity for the selection of the optimal evaluation tool for specific medical applications like organ transplantation. 


\section{References}

[1] Mühle R, Markgraf W, Hilsmann A, Eisert P, Wisotzky EL (2021) Comparison of Different Spectral Cameras for ImageGuided Surgery, submitted to Biomedical Optics Express
[2] Wisotzky EL, Kossack B, Uecker FC, Arens P, Hilsmann A, Eisert P (2020) Validation of two techniques for intraoperative hyperspectral human tissue determination, J. Med. Imaging 7(6):065001 https://doi.org/10.1117/1.jmi.7.6.065001 


\section{3rd International Workshop on Computer- Aided Diagnosis and Artificial Intelligence}

Chair: Hiroyuki Yoshida, PhD (US) 


\section{$\overline{\text { Feasibility study of MRI and multimodality CT/MRI }}$ radiomics for lung nodule classification}

\author{
A. Jatobá ${ }^{1}$, P. Azevedo-Marques ${ }^{2}$, M. Koenigkam-Santos ${ }^{2}$, \\ D. Wada ${ }^{2}$, M. Oliveira ${ }^{1}$ \\ ${ }^{1}$ Universidade Federal de Alagoas-UFAL, Programa de Pós- \\ Graduação em Informática, Maceió, AL, Brazil ${ }^{2}$ Ribeirao Preto \\ Medical School—University of Sao Paulo, Medical Imaging, \\ Hematology and Oncology, Ribeirao Preto, Brazil
}

Keywords multimodal medical imaging, radiomics, CT/MRI, lung nodule classification

\section{Purpose}

Lung cancer is the leading cause of cancer incidence and mortality worldwide, and Computed Tomography (CT) screening programs can contribute to its early detection. However, CT still has shortcomings: as this exam requires a considerable radiation dose, performing periodic examinations can become undesirable because of the risks of radiation-induced cancers.

In recent years, technical advancements made Magnetic Resonance Imaging (MRI) a viable modality for chest disease management. Besides not exposing the patient to radiation, MRI presents certain advantages over CT, such as superior soft-tissue contrast, allowing a better characterization of nodules. However, thoracic MRI still requires further clinical trials and protocol development to determine its actual capacities [1].

Multimodality medical imaging has been progressively applied in research and clinical practice. The intuition behind multimodality imaging is that different modalities can provide complementary information and better support for decision making and treatment [2].

This work's main goal was to assess whether MRI radiomics features are well-suited for lung nodules characterization and whether the combination of CT/MRI features can overcome its single modalities.

\section{Methods}

We acquired CT and MRI imaging from a cohort of 33 lung cancer patients. The sequences were obtained with patients in the supine position and with the aid of the deep inspiration breath-hold technique. The clinical chest MRI protocol included the post-contrast T1PC sequence. Our full image database comprises 33 nodules equal or greater than $10 \mathrm{~mm}$, of which 21 were diagnosed as malignant and 12 as benign. A senior radiologist pinpointed the nodules' location on the CT and T1PC sequences, and each lesion was segmented in both modalities using the semi-automatic segmentation algorithm FastGrowCut.

Next, We extracted a series of radiomics features from each nodule using the open-source library pyradiomics. At the time of this work, pyradiomics supported the following feature classes: First Order Statistics; Shape-based; gray level co-occurrence matrix (GLCM); gray level run length matrix (GLRLM); gray level size zone matrix (GLSZM); neighboring gray-tone difference matrix (NGTDM); and gray level dependence matrix (GLDM).

Since MRI has arbitrary intensity units, i.e., the grey level present in the images has no physiological meaning, image quantification with histogram-based features is impractical. Therefore, we discarded the first-order statistics features, leading to 89 metrics for each modality, divided into 14 shape-based, 24 GLCM, 16 GLRLM, 16 GLSZM, 5 NGTDM, and 14 GLDM features. For our multimodality CT/MRI approach, we combined the single modalities features into a new set containing 178 radiomic features. We scaled each feature using Min-Max scaling.

Due to the inherent high dimensionality of radiomics, it is essential to perform feature selection. We used a filter method by ranking the best features according to the ANOVA F-value statistic. For the decision tree and random forest classifier, we considered all features. For the remaining algorithms, we evaluated sets of 5, 10, 20, and 30 features. Because our dataset presents unbalanced classes, we balanced our cases using the synthetic minority over-sampling technique (SMOTE), evaluating $\mathrm{k}$ values of 3 and 5 for each classifier.

We selected a set of machine learning algorithms: logistic regression (LR); k-nearest neighbors (KNN); support vector machine (SVM); decision tree (DT); random forest (RF); naive Bayes (NB); and multi-layer perceptron (MLP). We performed hyperparameter optimization using grid-search with the AUC as the scoring metric. Moreover, we also measured each model's sensitivity and specificity.

We performed validation using fivefold nested cross-validation to ensure that no data leakage is happening within our optimization, repeating the experiment 30 times to obtain the average and deviation in performance for each metric.

\section{Results}

Figure 1 presents each classifier's average AUC performance for CT, T1PC, and CT + T1PC.

Our results contain an intriguing finding, as the models trained with T1PC radiomics presented superior performance compared to those trained with CT features. To assess the statistical significance of the classifiers' performance difference across the two datasets, we performed a Wilcoxon signed-rank test. We verified the significance of this difference and rejected the null hypothesis with a confidence level of $5 \%$ for Logistic Regression $(p=2.85 \mathrm{e}-4)$; SVM $(p=1.11 \mathrm{e}-4) ; \quad$ RF $\quad(p=5.57 \mathrm{e}-4) ; \quad$ and Naive Bayes $(p=2.36 \mathrm{e}-6)$. A conceivable explanation is that MRI's superior soft-tissue contrast allowed for a better characterization of the tumors in terms of radiomics features and led to a higher quality segmentation.

We can also observe that the combination of CT and T1PC features has not resulted in better classification performance, as T1PC surpassed the combined models in every case, except for the MLP classifier. This classifier, however, could not outperform the best classifiers in the T1PC set. This result may indicate that our feature selection approach was not suitable for combining multimodality radiomics features.

In general, our models have exhibited higher sensitivity than specificity. As our dataset contains more positive instances, the models could better learn the patterns for positive classification.

\section{Conclusion}

This study aimed to evaluate MRI and CT/MRI radiomics features' applicability to characterize lung nodules. Our results showed that MRI radiomics features could characterize lung nodules and support predictive models' development, with AUC values up to $17 \%$ higher than their CT counterparts. This advantage over CT is exciting, as MRI can enable a more in-depth investigation of a lung nodule's physiology. Moreover, MRI can mitigate radiation exposure and adverse reactions to contrast materials commonly used in CT.

On the other hand, our multimodality method has not proven advantageous, with lower performance than the single modalities models. Acknowledging that $\mathrm{CT}$ is the gold-standard modality for lung cancer diagnostic, we believe that a more sound investigation into multimodality medical imaging fusion techniques is needed.

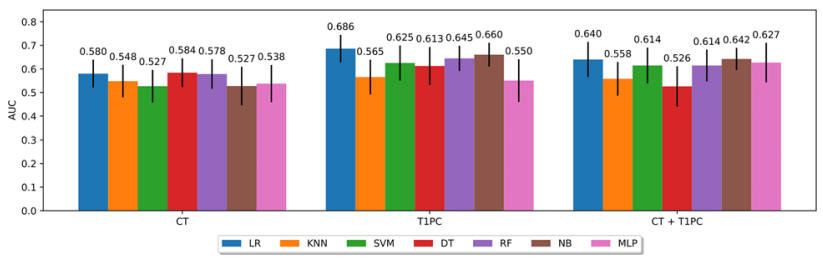

Fig. 1 Average AUC for each classifier, by imaging modality 


\section{Acknowledgements}

This study was financed in part by the Coordenacção de Aperfeiçoamento de Pessoal de Nível Superior (CAPES)—Finance Code 001 and by The São Paulo Research Foundation (FAPESP) - Grants \#16/17078-0 and 14/50889-7.

\section{References}

[1] Ohno Y, Kauczor HU, Hatabu H, Seo JB, van Beek EJ (2018) International Workshop for Pulmonary Functional Imaging (IWPFI). MRI for solitary pulmonary nodule and mass assessment: current state of the art. Journal of Magnetic Resonance Imaging, 47(6), 1437-1458.

[2] Wei L, Osman S, Hatt M, El Naqa I (2019). Machine learning for radiomics-based multimodality and multiparametric modeling. QJ Nucl Med Mol Imaging, 63(4), 323-338.

\section{Deep convolution neural network for laryngeal cancer classification on contact endoscopy-narrow band imaging via transfer learning}

E. Sharaf ${ }^{1}$, E. J. Gomes Ataide ${ }^{2}$, N. Esmaeili ${ }^{3,4}$, N. Davaris ${ }^{5}$, C. Arens 5 , M. Friebe 3,6

${ }^{1}$ Otto-von-Guericke University, Faculty of Electrical Engineering and Information Technology, Institute of Medical Technology (IMT), Magdeburg, Germany ${ }^{2}$ Otto-von-Guericke University, Clinic for Radiology and Nuclear Medicine, Department of Nuclear Medicine, Magdeburg, Germany ${ }^{3}$ Otto-von-Guericke University, Medical Faculty, INKA-Innovation Laboratory for Image Guided Therapy, Magdeburg, Germany ${ }^{4}$ Technical University Munich, Chair for Computer Aided Medical Procedures and Augmented Reality, Munich, Germany ${ }^{5}$ Magdeburg University Hospital, Department of Otorhinolaryngology, Head and Neck Surgery, Magdeburg, Germany ${ }^{6}$ IDTM GmbH, Recklinghausen, Germany

Keywords Deep Learning, Larynx, Contact Endoscopy, Classification

\section{Purpose}

A number of different imaging tools have been used in the past as a base for histopathological assessment of tumors in the larynx. White light endoscopy, Narrow Band Imaging (NBI), and Contact Endoscopy (CE) are widely accepted to visualize the region of the larynx. In spite of the technological advancements, differentiation between malignant and benign lesions in the larynx is difficult in reality, irrespective of the clinicians" level of experience. In addition, the subjectivity in laryngeal cancer diagnosis has been recorded, which reduces the confidence in the evaluation process by meter means of visual tools [1]. A higher accuracy requires a long and expensive training period.

This works investigated using Deep Convolutional Neural Networks (DCNN) for classifying 2-dimensional CE-NBI images into benign and malignant classes. The proposed approach aimed at introducing a fully automatic method with the objective orientation to classify the input images.

\section{Methods}

The work used a dataset of 8181 CE-NBI images as a base for the classification into benign or malignant classes. Images were resized to $284 \times 224$ pixels and normalized in the range $(0-1)$.

Instead of training a deep convolutional network from scratch, the method utilized a pre-trained network for classifying the images

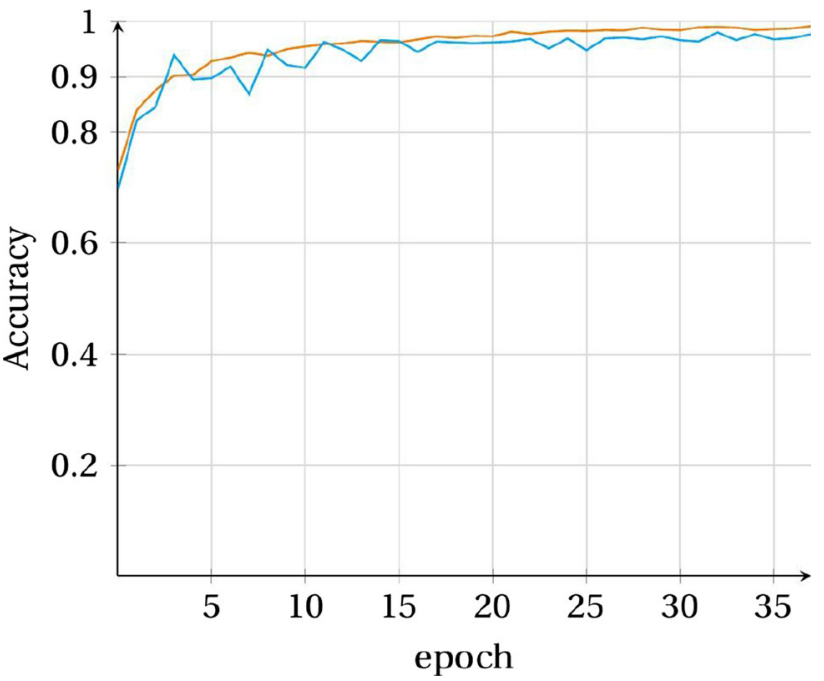

Fig. 1 Example of the model's performance (4th fold) after discarding deep layers of ReNet50. Training curve (orange line) and validation curve (blue line)

efficiently. ResNet50 [2] model was picked for being a well-vetted structure with an expected high success based on our literature research. The ResNet design overcomes the degradation problem shown in different convolutional networks" performance, which achieved a lower error rate with deeper layers. The method included transfer learning for accelerating the fine-tuning process of the network"s weights at the training phase. Transfer learning leads to a better generalization on validation images as the pre-trained model was trained on a larger number of images previously.

The implementation also included assessing the performance of portions of ResNet50, after cutting out deep layers, rather than taking the whole network ( $>23$ million weights), which has more learning capacity than needed for this application. This helped in reducing weights (parameters) count, to reduce the odds of model overfitting that happens in the case of neural networks, leading to a better generalization. For that, a "cut-off" layer was added to the conventional set of hyperparameters (optimizer, learning rate, loss function). Many models at different cut-offs were run and examined based on accuracy, sensitivity, and specificity metrics. Training and validation curves were inspected visually to examine the quality of convergence. A fivefold cross-validation technique was selected for assessing the performance at each trial (6545 images). Additionally, a testing phase was executed on 1636 unseen images for examining the generalizability of the model.

\section{Results}

The outcomes of the work showed that taking just a portion of the ResNet50 model achieved very good results with a $97.95 \%$ validation accuracy, against $96.75 \%$ for the complete provided ResNet50 model, with the same set of hyperparameters. On testing images, the smallersized network (after discard deeper layers) achieved 99.12\%, 98.92\%, $99.28 \%$, for accuracy, sensitivity, and specificity, respectively. Small structures were faster in the convergence than the complete pretrained model. Figure 1 shows the performance of a portion of ResNet50 (around $230 \mathrm{~K}$ weights) on the training and validation phases. As seen, the convergence was started at a high point, smoothly over the short course of epochs. The training and validation curves were moving forward to the optimal point in correspondence with no sign of overfitting. 


\section{Conclusion}

We conclude that the presented approach can be helpful in classifying CE-NBI images into benign and malignant classes objectively, especially with the current state of human-based diagnosis of laryngeal cancer being rather subjective. The high recognition rates indicate the robust performance by the suggested method, and the potential to have an objective diagnosis for helping the submission process in clinical practice, especially in difficult cases. Fine-tuning a portion of ResNet50 helps in decreasing the chance of overfitting, and requires less memory, with retaining high-performance metrics.

\section{References}

[1] Davaris N, Lux A, Esmaeili N, Illanes A, Boese A, Friebe M, Arens C (2020) Evaluation of Vascular Patterns using Contact Endoscopy and Narrow-Band Imaging (CE-NBI) for the Diagnosis of Vocal Fold Malignancy. Cancers 12(1): 248

[2] He K, Zhang X, Ren S, Sun J (2016). Deep Residual Learning for Image Recognition. In Proceedings of the IEEE Conference on Computer Vision and Pattern Recognition: 770-778.

\section{Deep convolutional neural networks (CNN) for COVID- 19 classification in chest $\mathrm{X}$-ray images with explainable artificial intelligence (XAI)}

X. Lessage ${ }^{1}$, S. Mahmoudi ${ }^{1}$, S. A. Mahmoudi ${ }^{1}$, S. Laraba ${ }^{1}$, O. Debauche $^{1}$, M. A. Belarbi ${ }^{1}$

${ }^{1}$ UMons, Faculty of Engineering, Computer Science, Mons, Belgium

Keywords X-ray analysis, classification, convolutional neural networks, covid-19

\section{Purpose}

The latest advances in machine learning and in particular with convolutional neurons $(\mathrm{CNN})$ have proven more than once their great accuracy in the detection of diseases $[1,2]$.

In this paper, we present a new approach for COVID-19 detection from chest X-ray images using Deep Learning algorithms. An efficient process consisting of techniques of transfer learning and a finetuning from pre-trained CNN models (InceptionV3, VGG16, MobileNet, EfficientNet, etc.) is proposed. A comparison of different architectures shows that VGG16 and MobileNet provide the highest scores: $97.5 \%$ and $99.3 \%$ of accuracy.

Experimentations have been conducted using an anonymized database from an Italian hospital thanks to a retrospective study. It is composed of three classes (normal, COVID-19, other pathologies) with a total number of 2905 images.

\section{Methods}

The goal of the proposed method consists of the separation of X-ray images into two or three categories by selecting and adapting the best CNN architecture. The classifier should be able to identify two (normal, COVID-19) or three classes (normal, COVID-19, other pathologies).

The dataset used was randomly splitted into three parts (70\% for training, $20 \%$ for validation, and $10 \%$ for test). We used data augmentation techniques to artificially increase the dataset and so significantly improve results accuracy.

We implemented a pre-trained architecture on ImageNet with several modifications such as: reduction of the number of classes in the last layer from 1000 to 3, integration of 5 dense layers to fine-tune the weights in a progressive way. During the training process, we used the training and validation datasets. Afterwards, we achieved our model test on an independant test dataset whose images were not seen by the neural network.

We evaluated six architectures (VGG16, MobileNet, Xception, InceptionV3, EfficientNetB0, DenseNet169) with the best parameters possible and we constated that the VGG16 and MobileNet architectures were more efficient.

In order to be able to explain the decision taken by the $\mathrm{CNN}$, we used a technique to visualise the pixels responsible for the classification. We opted for the GradCAM method which is frequently used in the domain of Explainable Artificial Intelligence (XAI) but there are many others.

Experiments were executed on a Linux cluster node with $32 \mathrm{CPU}$ cores using a single NVIDIA GeForce GTX 980 with 4 GB memory. Keras 2 with Tensorflow 1.8 backend was used as a deep learning framework.

\section{Results}

The analysis of Table 1 shows that MobileNet and VGG16 are the best models in terms of accuracy with the highest scores: $99.3 \%$ and $98.7 \%$ of accuracy respectively, $98.7 \%$ and $96.3 \%$ of sensitivity respectively, and $98.7 \%$ of specificity for both models.

For other architectures, the scores are acceptable but it is important to note that the number of false negatives is much higher.

In Figure 1, shown pixels responsible for the classification thanks to this temperature curve on the X-ray image and in particular in this case of COVID-19 suspicion.

\section{Conclusion}

Our approach based on in-depth learning is very promising for detecting pathologies, based on chest X-ray images. We recommend VGG16 and MobileNet that have achieved the best results in terms of precision, sensitivity and specificity.

Further investigations will be done using other datasets (and more specifically with CT images) in combination with various visualization methods.

Table 1 Comparison of models

\begin{tabular}{|c|c|c|c|c|c|c|c|c|c|c|c|c|}
\hline Models & $\begin{array}{l}\# \\
\text { Classes }\end{array}$ & $\begin{array}{l}\# \\
\text { Epochs }\end{array}$ & $\begin{array}{l}\text { \# Images } \\
\text { training }\end{array}$ & $\begin{array}{l}\text { \# Images } \\
\text { validation }\end{array}$ & $\begin{array}{l}\text { \# Images } \\
\text { test }\end{array}$ & $\begin{array}{l}\text { Accuracy } \\
\text { test }(\%)\end{array}$ & Sensibility (\%) & Specificity (\%) & \# TP & \# FP & \# FN & \# TN \\
\hline VGG16 & 3 & 48 & 2039 & 588 & 284 & 97.5 & 98.7 & 96.3 & 147 & 5 & 2 & 130 \\
\hline MobileNet & 3 & 48 & 2039 & 588 & 284 & 99.3 & 99.3 & 99.2 & 152 & 1 & 1 & 130 \\
\hline Xception & 3 & 48 & 2039 & 588 & 284 & 91.2 & 91.3 & 91.1 & 136 & 12 & 13 & 123 \\
\hline InceptionV3 & 3 & 48 & 2039 & 588 & 284 & 95.7 & 95.9 & 95.6 & 139 & 6 & 6 & 129 \\
\hline EfficientNetB0 & 3 & 48 & 2039 & 588 & 284 & 85.5 & 96.6 & 73.3 & 143 & 36 & 5 & 99 \\
\hline DenseNet 169 & 3 & 48 & 2039 & 588 & 284 & 90.1 & 85.9 & 94.8 & 128 & 7 & 21 & 128 \\
\hline
\end{tabular}



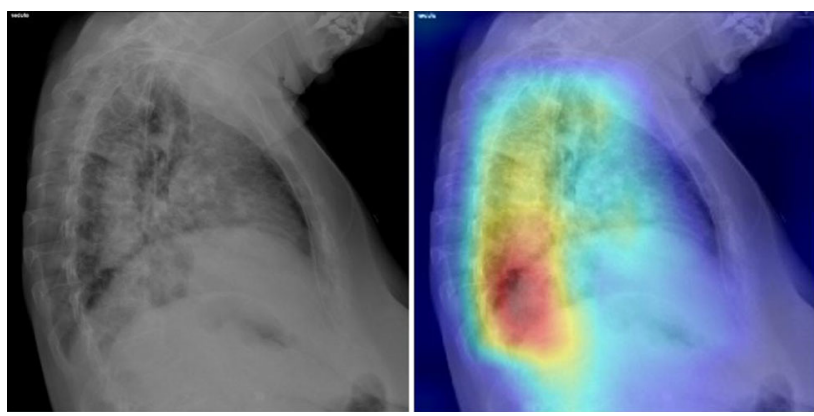

Fig. 1 VGG16 explanation with GradCAM method

\section{References}

[1] Hemdan EED, Shouman MA, Karar ME (2020) Covidx-net: A framework of deep learning classifiers to diagnose covid-19 in X-ray images (2020) Available via ArXiv. https://arxiv. org/abs/2003.11055. Cited 12 Aug 2020

[2] Apostolopoulos ID, Mpesiana TA (2020) Covid-19: automatic detection from X-ray images utilizing transfer learning with convolutional neural networks. Physical and Engineering Sciences in Medicine 43 635-640 (2020), https://doi.org/ $10.1007 /$ s13246-020-00865-4

\section{Weakly unsupervised contrastive unpaired learning for virtual bowel cleansing in computer-assisted diagnosis for laxative-free CT colonography}

J. Näppi ${ }^{1,2}$, H. Yoshida ${ }^{1,2}$

${ }^{1}$ Massachusetts General Hospital, Radiology, Boston, United States ${ }^{2}$ Harvard Medical School, Radiology, Boston, United States

Keywords Unsupervised learning, CT colonography, Electronic cleansing, Unpaired training

\section{Purpose}

Cathartic bowel preparation remains as a major barrier to colorectal screening. Laxative-free CT colonography bypasses the cathartic preparation in colorectal screening by combining the use of a lowfiber diet and an orally ingested fecal-tagging contrast agent with a computer-assisted diagnosis (CADx) method called electronic cleansing (EC) to perform virtual cleansing of the colon after the CT image acquisition. However, current EC methods generate 3D image artifacts that complicate the interpretation of the virtually cleansed images. In this pilot study, we investigated the feasibility of performing the $\mathrm{EC}$ in laxative-free CT colonography by use of contrastive unpaired learning.

\section{Methods}

Addressing the virtual cleansing problem can be formulated as learning an image-to-image translation, where the input image is a laxative-free CT colonography image volume and the output image is the corresponding virtually cleansed CT image volume. However, such an approach has two basic problems. First, clinical laxative-free CT colonography cases do not have precisely matching natural virtually cleansed versions that could be used as paired training samples. Second, the relationship between the input and output images is a surjection, because the virtually cleansed output image has an infinite variety of corresponding fecal-tagged input images. Although the first problem can be addressed by use of the unpaired training method provided by cycle-consistent generative adversarial networks (CycleGANs) [1], the cycle-consistency condition assumes that the relationship between the input and output images is a bijection, thereby failing to address the second problem.

Therefore, in this study, we made use of weakly unsupervised 3D contrastive unpaired training (3D CUT), which is based on maximizing the mutual information between unpaired input and output images sampled from two domains [2]. The method is implemented in terms of a 3D GAN, where the generator that is trained to generate the EC images is composed of sequentially applied encoder and decoder networks. The encoder is trained to pay attention to common elements between the input and output image domains while being invariant to their differences, whereas the decoder is trained to synthesize the domain-specific features. The discriminator of the 3D GAN is trained to differentiate between real and synthetically generated EC images.

For a preliminary evaluation, we collected unpaired samples of 147 fecal-tagged laxative-free CT colonography cases and 147 cathartically cleansed untagged CT colonography cases. The laxative-free CT colonography preparation involved oral ingestion of a low-osmolar, non-ionic iodinated agent for fecal tagging in combination with a lowfiber diet, and the CT colonography scans were acquired by use of multidetector CT scanners in supine and prone position with $120 \mathrm{kVp}$ and $50 \mathrm{mAs}$ effective at a maximum 2.5 -mm z-axis length with 1.25 $\mathrm{mm}$ overlap interval. The cathartic untagged CT colonography examinations were prepared with a standard pre-colonoscopy cleansing without using fecal tagging, and the CT colonography scans were acquired by use of helical $\mathrm{CT}$ scanners in supine and prone position with $120 \mathrm{kVp}$ and $60-100 \mathrm{~mA}$ at $2.5-5.0 \mathrm{~mm}$ collimation and reconstruction intervals of $1.5-2.5 \mathrm{~mm}$. A training dataset was established by extraction of $128 \times 128 \times 128$-voxel training volumes of interest (VOIs) at an isotropic $0.625-\mathrm{mm}$ voxel resolution from both types of CT colonography cases. An independent external test dataset was established by the sampling of additional laxative-free CT colonography examinations that were not part of the 147 laxative-free training cases. After the training, the 3D CUT was tested with the independent test dataset to evaluate if it had been able to learn to perform EC based on the unpaired training. As a reference method, we also trained and tested a 3D CycleGAN in a similar manner.

\section{Results}

The 3D CUT and the reference 3D CycleGAN were trained with 9735 laxative-free VOIs and 10,152 untagged cathartic VOIs. However, the 3D CycleGAN did not produce meaningful test output.

Figure 1a shows an example of the result of the testing of the 3D CUT on a laxative-free test case. As can be seen from the images, the

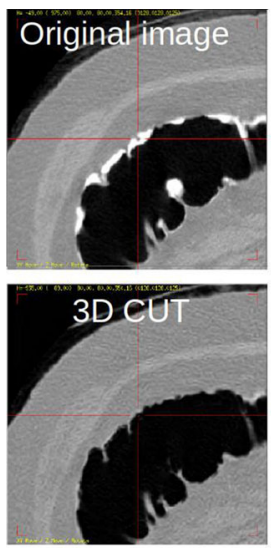

(a)

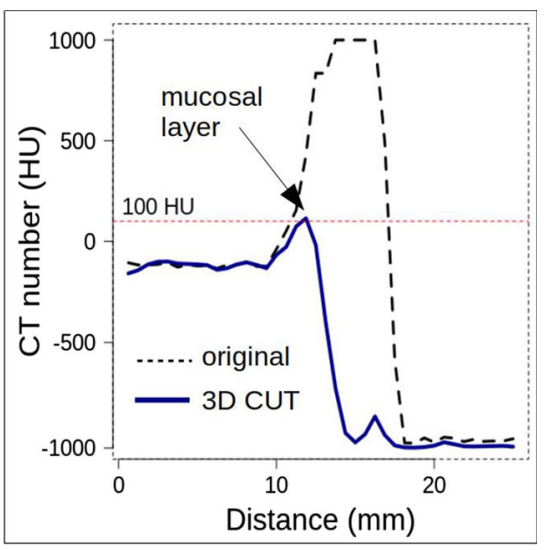

(b)
Fig. 1 a An example of an axial CT image before (top) and after (bottom) performing EC by the 3D CUT. b Plot of a line profile of the bowel surface before (black dotted line) and after (blue line) performing the EC by the 3D CUT 
3D CUT was able to subtract semi-solid residual tagged feces realistically from the laxative-free CT colonography images.

Figure $1 \mathrm{~b}$ shows a line profile of the bowel surface before and after the EC. The plot demonstrates that the 3D CUT was able to subtract the tagged feces adhering to the bowel wall and also able to reconstruct the mucosal thickening layer of the bowel wall. The surrounding soft-tissue and lumen regions retained realistic radiodensity values.

We also noticed some problems. In regions that contained large pools of fecal-tagged fluid, the 3D CUT could convert the tagged fluid into untagged fluid rather than subtract the fluid. This happened because the cathartically cleansed untagged CT colonography training cases contained large quantities of untagged fluid. Thus, while the 3D CUT did learn to perform EC of semi-solid tagged feces, it also learned to convert tagged fluid into untagged fluid based on the examples of the training cases. Resolving such issues provides topics for follow-up studies.

\section{Conclusion}

We performed a pilot study to investigate the feasibility of performing contrastive unpaired learning for EC in laxative-free CT colonography. The 3D CUT model was trained with unpaired samples of laxative-free CT colonography and untagged cathartic CT colonography cases. Our preliminary results indicate that the resulting model can be used to perform EC in laxative-free CT colonography. However, our study also revealed some issues to be addressed in follow-up studies.

\section{Acknowledgements}

This study was supported by the National Institute of Biomedical Imaging and Bioengineering and National Cancer Institute of the National Institutes of Health under Award Number R21EB024025, R01EB023942, and R01CA212382.

\section{References}

[1] Näppi JJ, Yoshida H (2019) Cycle-consistent 3D-generative adversarial network for virtual bowel cleansing in CT colonography. Proc SPIE 10949, Medical Imaging 2019: Image Processing, 1094922Z

[2] Park T, Efros AA, Zhang R, Zhu J-Y (2020) Contrastive learning for unpaired image-to-image translation. 16th European Conference on Computer Vision (ECCV)

\section{Computer assisted pneumonia quantification in COVID-19: data from the CovILD study}

A. Luger ${ }^{1}$, C. Schwabl ${ }^{1}$, T. Sonnweber ${ }^{1}$, S. Sahanic ${ }^{1}$, A. Pizzini ${ }^{1}$, A. Böhm $^{1}$, J. Löffler-Ragg ${ }^{1}$, I. Tancevski ${ }^{1}$, G. Feuchtner ${ }^{1}$, M. Johannidis $^{1}$, R. Kirchmair ${ }^{1}$, E. Wöll ${ }^{1}$, G. Weiss ${ }^{1}$, E. Gizewski ${ }^{1}$, G. Widmann ${ }^{1}$

${ }^{1}$ Medical University of Innsbruck, Innsbruck, Austria

Keywords COVID-19, quantification, computed tomography, pneumonia

\section{Purpose}

Computed tomography (CT) is the imaging method of choice in patients with severe acute respiratory syndrome coronavirus 2 (SARS-CoV-2) disease 2019 (COVID-19), worsening of symptoms and detection of persistent lung abnormalities after recovery. Subjective scores currently classify CT findings based on severity of pulmonary involvement (from mild ground glass opacities (GGO) to marked consolidations) or estimate the amount of these findings. Estimation of the percentage of involved lung parenchyma is time consuming and prone to inter-reader variability. Computer assisted pneumonia quantification software may provide an objective alternative. The prospective, multicentre, observational CovILD study (development of interstitial lung disease (ILD) in patients with severe SARS-CoV-2 infection) systematically evaluated the persisting cardio-pulmonary damage of COVID-19 patients 60 and 100 days after COVID-19 onset. CT scans from this study were used to evaluate a prototype software for pneumonia quantification.

\section{Methods}

The trial protocol was approved by the institutional review board at Innsbruck Medical University (EK Nr: 1103/2020) and was registered at ClinicalTrials.gov (registration number: NCT04416100). Informed consent was obtained from each patient. CT scans in low-dose setting (100 kVp tube potential) were acquired without ECG gating on a 128 slice multidetector CT hardware with a $128 \times 0.6 \mathrm{~mm}$ collimation and spiral pitch factor of 1.1. Overall, pulmonary findings were graded for every lobe using a modified CT severity score by consensus reading of two pulmonary radiologists: 0-none, 1-minimal (subtle GGO), 2-mild (several GGO, subtle reticulation), 3-moderate (multiple GGO, reticulation, small consolidation), 4-severe (extensive GGO, consolidation, reticulation with distortion), and 5-massive (massive findings, parenchymal destructions). The maximum score was 25 (i.e. maximum score 5 per lobe). Syngo.via CT Pneumonia Analysis (Siemens Healthineers, Erlangen, Germany) research prototype for the detection and quantification of abnormalities consistent with pneumonia was used to calculate the percentage of opacity (indicating GGO), and percentage of high opacity (indicating consolidation). Correlations between CT severity score and softwarebased pneumonia quantification were assessed with Spearman rank test.

\section{Results}

In 145 COVID-19 patients, CT lung abnormalities typical for COVID-19 were found in $77 \%$ of patients at visit 1 and in $63 \%$ of individuals at visit 2 . The CT severity score unveiled a moderate structural involvement in most patients at 60-day follow-up and a mean score of 8 points (IQR 2-15). Software based pneumonia quantification revealed a mean percentage of opacity of $0.32 \%$ (IQR $0.01-3.91$ ), and mean percentage of high opacity of $0.23 \%$ (IQR $0.00-0.12$ ) at 60-day follow-up, and the software-based quantification and the CT severity scoring by two radiologists demonstrated a high correlation (correlation coefficient $=0.8, p<0.001$ ). The majority of participants $(81 \%)$ demonstrated an improvement in both the CT severity score and the software-based pneumonia quantification 100 days after COVID-19 onset.

Conclusion

Software-based pneumonia quantification may show a high correlation to a subjective CT severity score. It is uninfluenced by observer and objectives quantification of ground glass opacification and consolidation.

\section{Parametric identification of prostate shape using the superellipses and its correlation with pathology}

\author{
Y. Onodera ${ }^{1}$, N. Koizumi ${ }^{1}$, Y. Shigenari ${ }^{1}$, R. Igarashi ${ }^{1}$, Y. \\ Nishiyama $^{2}$, S. Shoji ${ }^{3}$ \\ ${ }^{1}$ The University of Electro-Communications, Department \\ of Mechanical and Intelligent Systems Engineering, Chofu, Japan \\ ${ }^{2}$ The University of Electro-Communications, Department \\ of Computer and Network Engineering, Chofu, Japan ${ }^{3}$ Tokai \\ University, Department of Urology, Isehara, Japan
}

Keywords superellipse, benign prostatic hyperplasia, SVM, CAD 


\section{Purpose}

In recent years, due to the increase in the number of patients associated with the aging population, emphasis has been placed on prevention and early detection of diseases as a way of medical care. One disease that has a high incidence among elderly men is benign prostatic hyperplasia (BPH). BPH is a disease specific to elderly men in which the enlargement of the internal glands surrounding the urethra causes pressure on the urethra, leading to dysuria and a decrease in QOL [1].

In this study, we focused on imaging diagnosis, which is relatively common, and aimed at diagnosing benign prostatic hyperplasia from the morphological characteristics of the prostate gland, which is incidentally visualized by MRI and abdominal CT, in order to realize a simple screening test. In this paper, we propose a diagnostic prediction system for prostate MRI and evaluate its diagnostic performance.

\section{Methods}

The proposed system is constructed in two major stages. The outline of the proposed system is summarized in Fig. 1. In the first stage, we extract features for classification from input images. The method of feature extraction is described below. First, the prostate contour depicted on the prostate MRI under the guidance of a doctor is treated as the correct contour. We scan the pixels on the prostate MRI to obtain the coordinates of the correct contour. Twenty-four points are obtained at equal angles from the center of the image, and 100 spline interpolation points are used to obtain smooth and continuous coordinates. Based on the acquired coordinates, a mathematical model called superellipses is used for fitting. In the prostate shape extraction, we use a hyperelliptic model with eight deformable parameters $P=\{1 x, l y, r, s y, s q, x y, t, b\}$ by Gong et al. [2]. Among the eight hyperelliptic parameters $\mathrm{P}=\{\mathrm{lx}, \mathrm{ly}, \mathrm{r}, \mathrm{sy}, \mathrm{sq}, \mathrm{xy}, \mathrm{t}, \mathrm{b}\}$, the position parameter $\mathrm{P} p=\{1 \mathrm{x}, \mathrm{ly}, \mathrm{r}, \mathrm{sy}\}$ is calculated numerically and the shape parameter $P s=\{s q, x y, t, b\}$ is identified using grid search.

In the second stage, a classifier is trained using the feature data set obtained in the first stage, and the trained classifier is used to diagnose whether or not the test data has benign prostatic hyperplasia. The standardized feature data set obtained in the first stage is used as the explanatory variable and the prostate pathology as the objective function (normal $=0, \mathrm{BPH}=1$ ), which is input to the SVM model to build the learned model. The parameters are tuned using the grid search method and $\mathrm{k}$-cross partitioning validation to prevent overtraining and improve the generalization performance of the model.

Initially, we classify the pathological conditions using 4-dimensional features with only the shape parameter Ps. Based on the results, we then experimented with a six-dimensional feature set that includes the shape parameter Ps plus "sy" and the area encompassed by the prostate contour (number of pixels) "S". We use prostate MRI as the

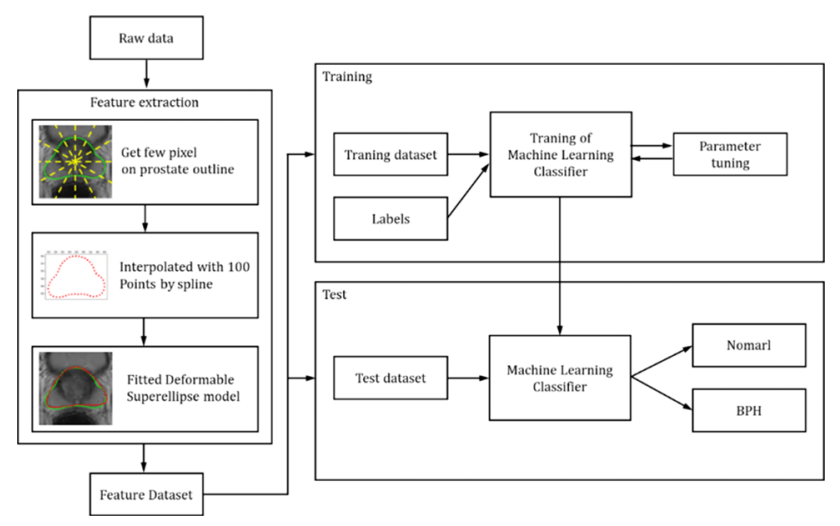

Fig. 1 Proposed algorithm outline
Table 1 Result of experiment

\begin{tabular}{lll}
\hline & Mean & Standard \\
\hline Accuracy & 0.961 & 0.035 \\
Precision & 0.971 & 0.038 \\
Recall & 0.951 & 0.056 \\
F-score & 0.96 & 0.036 \\
AUC & 0.96 & 0.176 \\
\hline
\end{tabular}

training and diagnostic data for the system evaluation experiments. Four consecutive prostate MRI images were used per patient, focusing on the area with the largest endoglandular area in the 3-mm slice T2-weighted images. Only images that show typical morphological features and that are equivalent to the diagnostic results and the physician's imaging results will be used in this experiment.

\section{Results}

The results of this experiment using 6-dimensional features are shown in the Table 1; the results using 4-dimensional features show that the correct answer rate, fit rate, recall rate, F value, and AUC are 0.771, $0.749,0.826,0.781$, and 0.789 , respectively. Then, for the diagnostic system using 6-dimensional features, the correct answer rate, fit rate, recall rate, F value, and AUC were 0.961, 0.971, 0.951, 0.960, and 0.960 , respectively, as shown in the table. In this experiment, the morphological features using the hyperelliptic model are considered to be effective features for the diagnosis of benign prostatic hyperplasia with ideal morphology, since we have shown that features with relatively low dimensionality in this experiment produce a correct answer rate of nearly $80 \%$. Since low dimensionality was thought to be the cause of the misidentification, experiments were conducted with additional features, and the accuracy was improved.

\section{Conclusion}

In this study, we evaluated a method for predicting the diagnosis of benign prostatic hyperplasia (BPH) using morphological features based on superellipses and machine learning to realize a diagnostic system from imaging in screening tests for BPH. We conducted experiments using the shape deformation parameter of the superellipses, and confirmed the effectiveness of the method by proposing new features, since problems in diagnosis were confirmed. In the future, we would like to improve the discriminative performance of the model by extracting more features, and introduce algorithms to reduce the number of missed pathologies, such as the introduction of multi-step diagnosis using the confidence level. In addition, there are some problems to be solved, such as the variation of the fitting performance depending on the setting of the reference axis of the superellipses, and the large computation time required for the grid search method, so we would like to automate and speed up the superellipses fitting.

\section{References}

[1] AUA Practice Guidelines Committee (2003) AUA Guideline on Management of Benign Prostatic Hyperplasia (2003). The journal of Urology 170(2):530-547

[2] Gong L, Pathak SD, Haynor DR, Cho PS, Kim Y (2004)Parametric Shape Modeling Using Deformable Superellipsess for Prostate Segmentation. IEEE Transactions on Medical Imaging 23(3):340-349 


\section{Predicting interstitial and Covid-19 diseases on chest $\mathrm{X}$-ray using a convolutional neural network with transfer learning}

L. L. Lima ${ }^{1,2}$, J. C. Nather $\mathrm{Jr}^{1}$, M. Koenigkam-Santos ${ }^{1,2}$, L. Peron ${ }^{1}$, K. L. Fontes Lopes ${ }^{1}$, C. Machado ${ }^{1}$, F. Sarmento-Neto ${ }^{1}$, D. Wada ${ }^{1}$, M. Koenigkam-Santos ${ }^{1}$, P. Azevedo-Marques ${ }^{1,2}$

${ }^{1}$ Ribeirao Preto Medical School-University of Sao Paulo, Medical Imaging, Hematology and Oncology, Ribeirao Preto, Brazil

${ }^{2}$ University of São Paulo, Interunit Graduate Program

in Bioengineering, São Carlos, Brazil

Keywords Lung Interstitial Disease, Covid-19, Convolutional Neural Network, Transfer Learning

\section{Purpose}

Lung interstitial diseases (LID) includes a group of more than 200 chronic lung disarrangements, which can reduce the ability of the air sacs to capture and carry oxygen into the bloodstream and consequently lead to permanent loss of the ability to breathe [1]. Autoimmune diseases, genetic abnormalities, and long-term exposures to hazardous materials can cause these diseases [1]. Another disease is the Coronavirus disease 2019 (Covid-19), highly contagious has spread rapidly throughout the world since January of 2020 [2]. According to the World Health Organization (WHO), the mortality rate reaches $2-3 \%$ of people affected by Covid-19 [2].

Chest X-ray is often the initial exam in the face of clinical suspicion of lung diseases, in addition to being the simplest, cheapest, and most available imaging exam. To help specialists improve the diagnostic accuracy of LID and Covid-19 diseases presented on chest $\mathrm{X}$-ray images by acting as a second opinion through a computersupplied suggestion, computer-aided diagnosis (CAD) systems has been developed.

One machine learning technique that has emerged to improve CAD systems is the convolutional neural network (CNN) that is based on deep learning $[1,2]$. CNN extracts the image"s features, selects the most important features, and classifies the input images. Nevertheless, modeling the best $\mathrm{CNN}$ architecture for any specific problem by hand can be an exhausting, time-consuming, and expensive task. However, an alternative is the use of the Transfer Learning technique, which uses a network pre-trained on a large dataset applied on a new dataset. In this work, we performed a CNN analysis with a transfer-learning algorithm to predict LID and Covid-19 diseases on chest X-ray images.

\section{Methods}

This was a retrospective study approved by our institutional review board with a waiver of patients' informed consent. Frontal chest $\mathrm{X}$-ray images of patients diagnosed in three groups have been used: healthy (380 images), with LID (310 images), and with Covid-19 (199 images). All images were previously analyzed by a thoracic radiologist and covid-19 diagnosis was confirmed by RT-PCR. Figure 1 shows an example from the Covid-19 set. Original DICOM images were converted to PNG with 3 channels (RGB), to augment the database and improve CNN"s performance in the experiments. Next, pixel values were normalized between 0 and 1 .

A balanced subset of images was also randomly organized with 199 cases from each group, that was splitted in $80 \%$ for training (159 samples from each group) and $20 \%$ for testing (40 samples from each group). An unbalanced testing was also performed using 222 images from healthy, 151 images from LID groups, and 40 images from Covid-19 set.

The experiments were performed using a graphics-processing unit (GPU) NVidia Titan X with 12 Gigabytes of RAM, 3584 cores, speed of $1.5 \mathrm{GHz}$, and Pascal Architecture. For training, the samples were shuffled for tenfold cross-validation using CNN through the

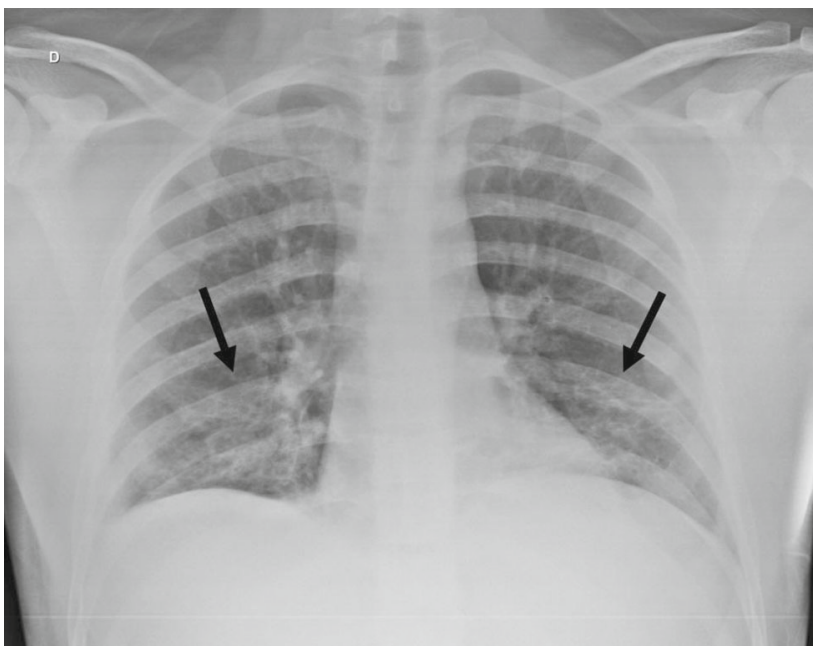

Fig. 1 Frontal CXR of a patient with COVID-19 pneumonia showing most typical interstitial, hazy opacities, predominantly involving basal and peripheral areas of the lungs (arrows). As all patients included in this study, diagnosis was confirmed by RT-PCR and images were previously analyzed by a thoracic radiologist

framework Tensorflow (v.2.1.0.). Through the technique of transfer learning, the pre-trained network used was the VGG19, so the resolution of the images was fixed in $224 \times 224$. From the VGG19, all layers until the block4_pool layer were frozen preventing their weights from being updated during the training, and the layers until block5_pool were retrained. Next, some layers were added: GlobalAveragePooling2D, BatchNormalization, a dense layer with 128 units with the activation function ReLU, a dropout layer with the rate of 0.6, and the final dense layer with 3 units for the classification of the three classes with Softmax activation function. The values of batch size and epoch were 36 and 100, respectively. The optimizer function used was Adam to minimize the categorical cross-entropy. The processing time for training was $50 \mathrm{~min}$.

To evaluate the performance of testing cases the statistical metrics used were the area under the receiver operating characteristic curve (AUC), sensitivity, specificity, and accuracy. We evaluated the metrics sensitivity, specificity, and accuracy considering each class as a positive at a time. The confidence interval (CI) used was $95 \%$.

\section{Results}

In the evaluation using the balanced testing set, results considering the healthy class as positive were sensitivity of $85 \%$ (CI: $0.69-0.93$ ), specificity of $92.50 \%$ (CI: $0.83-0.96$ ), and accuracy of $90 \%$ (CI: $0.82-0.94)$. Results for the LID class as positive were sensitivity of $80 \%$ (CI: $0.63-0.90$ ), specificity of $96.25 \%$ (CI: 0.88-0.99), and accuracy of $90.83 \%$ (CI: $0.83-0.95$ ); and for the Covid-19 class as positive results were sensitivity of $92.50 \%$ (CI: 0.78-0.98), specificity of $90 \%$ (CI: $0.80-0.95$ ), and accuracy of $90.83 \%$ (CI: 0.83-0.95). The AUC values were 0.96, 0.97, and 0.97 for classes healthy, LID, and Covid-19, respectively.

In the evaluation using the unbalanced testing set, the results considering the healthy class as positive were sensitivity of $84.23 \%$ (CI: $0.78-0.88$ ), specificity of $91.62 \%$ (CI: $0.87-0.95$ ), and accuracy of $87.65 \%$ (CI: $0.83-0.90$ ). Results for the LID class as positive were sensitivity of $84.77 \%$ (CI: $0.77-0.89$ ), specificity of $93.13 \%$ (CI: $0.89-0.95$ ), and accuracy of $90.07 \%$ (CI: 0.86-0.92); and for the Covid-19 class as positive results were sensitivity of $92.50 \%$ (CI: $0.78-0.98$ ), specificity of $92.76 \%$ (CI: $0.78-0.98$ ), and accuracy of 92.74\% (CI: 0.89-0.94). The AUC values were 0.95, 0.94, and 0.97 for classes healthy, LID, and Covid-19, respectively. 


\section{Conclusion}

This study performed a CNN modeling integrated with a transfer learning architecture for the prediction of chest X-ray images in healthy, LID, or Covid-19. The small number of cases is a clear limitation of this study. Even so, the proposed approach presented potential to be used as a tool for classifying chest X-ray images. Moreover, our method does not require image segmentation, use of handcrafted features extractors, or clinical features as prerequisites. Acknowledgements

This study was financed in part by the Coordenacção de Aperfeiçoamento de Pessoal de Nível Superior (CAPES)—Finance Code 001 and by The São Paulo Research Foundation (FAPESP) - Grants \#2020/07200-9, \#16/17078-0 and 14/50889-7. We thank the Tecgraf/ PUC-Rio Institute for the computational resources made available, being of vital importance for the conclusion of this work.

\section{References}

[1] Anthimopoulos M, Christodoulidis S, Ebner L, Christe A, Mougiakakou S (2016) Lung Pattern Classification for Interstitial Lung Diseases Using a Deep Convolutional Neural Network. IEEE Trans Med Imaging 35(5):1207-1216. https://doi.org/ 10.1109/tmi.2016.2535865. Epub 2016 Feb 29.

[2] Kamil MY. A deep learning framework to detect Covid-19 disease via chest $\mathrm{X}$-ray and $\mathrm{CT}$ scan images. International Journal of Electrical and Computer Engineering. 11. 2021;11(1)844-850. https://doi.org/10.11591/ijece.v11i1. pp. 844-850.

\section{Breast cancer detection in digital mammography with convolutional neural network: retrospective study in Belgium}

\author{
S. Mahmoudi ${ }^{1}$, X. Lessage ${ }^{1}$, F. Giudice ${ }^{1}$, S. Murgo ${ }^{2}$, S. \\ A. Mahmoudi ${ }^{1}$ \\ ${ }^{1}$ University of Mons-Faculty of Engineering, Computer Sciences \\ Department, Mons, Belgium ${ }^{2}$ CHR de Mons, Radiology, Mons, \\ Belgium
}

Keywords Breast cancer, Mammography, Classification, convolutional neural networks

\section{Purpose}

Nowadays, cancer is one of the leading causes of human death in the world. In 2020, breast carcinoma represented $11.7 \%$ of cancers reported in the female world population. Medical imaging is crucial for breast cancer screening and occupies a large place in diagnosis. Early screening with digital mammography is the key factor of successful detection of breast cancer. Also, automatic tumor detection could help radiologists in their work and them to achieve fast and accurate diagnosis. We propose in this work, a deep learning-based classification method to assist radiologists in the context of breast cancer screening and diagnosis. This system is able to classify digital mammograms into two categories: negative or benign and malignant. The main objective of this automatic imaging based detection method is to avoid human second reading and at the same time reduce radiologists workload. Several deep convolutional neural networks architectures were assessed in this work, and a three stage training process was proposed. Our experiments were conducted by using a real database collected from the CHR Mons-Hainaut in Belgium composed of 2410 images including 378 malignant cases.

Methods

Recently, a lot of research projects were conducted to assess early detection of breast cancer, either with masses or microcalcification detection. However a large majority of the research projects in this field $[1,2]$ were based on publicly available databases like CBISDDSM, INbreast or MIAS. These databases could be used to assess automatic tumor detection approaches but could not fit to local or regional populations and their specificities. The main objective of our work is to propose an efficient mammography image classifier using a real database collected from CHR Mons-Hainaut hospital in Belgium and an adapted pre-trained convolutional neural network. The proposed method consists of developing a binary classifier using deep neural networks applied to mammography images. The classifier is able to identify two classes: malignant and benign in order to assist the radiologist in his diagnosis. The dataset used was randomly splitted into three parts (70\% for training, $20 \%$ for validation, and $10 \%$ for test). We used data augmentation techniques to artificially increase the dataset and so significantly improve results accuracy. During the development of our model the training strategy was defined by implementing transfer learning from pre-trained architectures with ImageNet database and fine-tuning techniques. We tested the following CNN pre-trained architectures: ResNet50, ResNet152, InceptionV3, ResNet101, AlexNet, VGG19, VGG16. We can note that pretrained networks allow faster training and improve the generalization capacity of the network compared to random initialization. Also, it is worth mentioning that pretrained networks present in their bottom layers the main primitive features that tend to be universal and common to the majority of the datasets. On the other side, top layers represent high level descriptive features related to final labels. Hence, finetuning could be achieved in depth for the top layers. So, applying a higher learning rate for top layers could take into account this fact. That is why; we propose in our work a three stage training framework similar to the computational framework proposed by Shen li et al. in [1]. So, our learning strategy freezes in the first stage all parameter layers except the last one. This step allows to finetune the model by using only the last layer. We use in this stage a learning rate of 0.001 for 3 epochs. In a second stage we unfreeze top layers and apply a learning rate of 0.0001 for 10 epochs. Finally, we train the whole network with a learning rate of 0.00001 for 50 epochs combined to an early stopping condition. Also, in order to overcome overfitting we added a dropout layer with a probability set to 0.5 . On the other hand, Stochastic gradient descent (SGD) and Adam algorithms were both used in our tests to optimize the networks.

The proposed method was tested with a real database composed of 2410 images from CHR Mons-Hainaut taken in 2016 and containing negative or benign and malignant cases with verified pathological information. This dataset includes 378 mammograms with cancer (malignant cases). The proportion between benign and malignant cases was chosen to maintain the frequency of occurrence of breast cancer in real life. During the training process, we used the training and validation datasets. Afterwards, we achieved our model test on an independent test dataset whose images were not seen by the neural network.

Results

We present in Table 1 the results obtained with our binary classifier for the different CNN architectures tested with the Adam optimizer, a batch size equal to 16 and a Dense layer size of 512. The ES column is EarlyStopping parameter and AUC is the area under the ROC curve. The third column from the left corresponds to the number of top layers trained in the second training phase. These results show that the three best results were obtained with AlexNet, VGG19 and VGG16. For VGG16 architecture, training and validation accuracy was respectively $99.47 \%$ and $98.96 \%$, while test accuracy reached 97.10\%, and AUC $93.1 \%$.

In a second experimentation we kept the VGG16 model which has shown the best results and we have applied a final configuration with EarlyStopping and a Dense layer size of 128. In this final experimentation, we increased the batch size to 32 and applied SGD optimizer. Figure 1 shows the accuracy convergence at the end of the 
Table 1 Accuracy results for the different proposed architectures

\begin{tabular}{|c|c|c|c|c|c|c|}
\hline Modèle & ES & Top Layers & Train_acc $(\%)$ & Val_acc (\%) & Test_acc $(\%)$ & AUC (\%) \\
\hline InceptionResNetV2 & 6 & 48 & 99.94 & 84.23 & 79.67 & 52.00 \\
\hline ResNet50 & 11 & 46 & 100.00 & 84.23 & 79.67 & 52.00 \\
\hline ResNet 152 & 8 & 46 & 100.00 & 84.44 & 79.67 & 52.00 \\
\hline InceptionV3 & 6 & 48 & 99.88 & 85.68 & 81.33 & 55.90 \\
\hline ResNet101 & 12 & 46 & 100.00 & 92.32 & 86.31 & 67.60 \\
\hline AlexNet & 10 & 11 & 98.52 & 95.23 & 93.36 & 84.30 \\
\hline VGG19 & 8 & 13 & 99.47 & 98.96 & 96.68 & 92.20 \\
\hline VGG16 & 8 & 11 & 99.47 & 98.96 & 97.10 & 93.10 \\
\hline
\end{tabular}

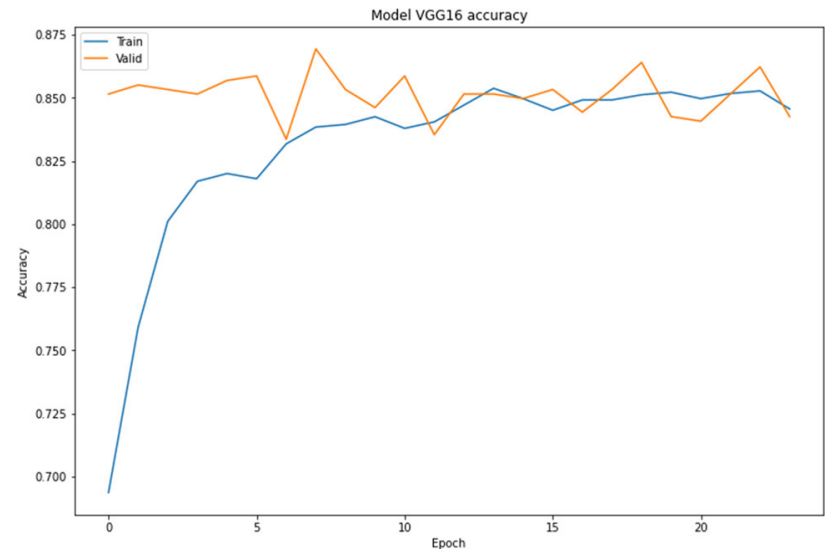

Fig. 1 training and validation accuration evolution at the third training stage

third training phase. After a total of 30 epochs, the training accuracy reached $98.34 \%$ and the validation was slightly higher with a value of $99.17 \%$.

For our test dataset the achieved accuracy was of $98.34 \%$ and the AUC of $96.1 \%$. The confusion matrix obtained with the VGG16 classifier generates a very few number of false negatives $(\mathrm{FN}=4$, $\mathrm{FP}=0, \mathrm{TP}=47, \mathrm{TN}=190$ ). Indeed, out of a total of 51 cancer cases in the test dataset, the model missed only four ones. In addition, all benign class images were properly classified.

\section{Conclusion}

In this paper, we addressed the problem of breast cancer detection by using digital mammography and deep convolutional neural networks. Several pre-trained CNN architectures were used such as: ResNet50, ResNet152, InceptionV3, ResNet101, AlexNet, VGG19, VGG16. The major contribution of this paper is the use of a real database, and the use of an adapted pre-trained model based on a three stage training process. The adapted VGG16 model achieved the best accuracy score of $98.34 \%$ for the test dataset. This result overcomes the latest results that use CBIS-DDSM database and present encouraging steps to automate breast cancer detection in hospitals. Further investigations will be done using a bigger dataset in order to improve screening results.

\section{References}

[1] Shen L, Margolies L, Rothstein J, Fluder E, McBride R, Sieh W (2019) Deep learning to improve breast cancer detection on screening mammography. Scientific Reports, 9:1-12. doi: https://doi.org/10.1038/s41598-019-48995-4.
[2] Tsochatzidis L, Costaridou L, Pratikakis I (2019) Deep learning for breastcancer diagnosis from mammograms-a comparative study. Journal of Imaging, 5:37. https://pubmed.ncbi.nlm. nih.gov/31467326.

\section{A deep learning scheme for predicting the cognitive status of patients with small vessel disease and mild cognitive impairment}

S. Atnafu ${ }^{1}$, C. Marzi ${ }^{1}$, E. Salvadori ${ }^{2}$, A. Poggesi ${ }^{2}$, A. Giorgio $^{3}$, N. De Stefano $^{3}$, M. Mascalchi ${ }^{4}$, L. Pantoni ${ }^{5}$, S. Diciotti ${ }^{1,6}$

${ }^{1}$ University of Bologna, Department of Electrical, Electronic, and Information Engineering "Guglielmo Marconi", Bologna, Italy ${ }^{2}$ University of Florence, NEUROFARBA Department, Neuroscience Section, Florence, Italy ${ }^{3}$ University of Siena, Department of Medicine, Surgery, and Neuroscience, Siena, Italy ${ }^{4}$ University of Florence, "Mario Serio" Department of Experimental and Clinical Biomedical Sciences, Florence, Italy ${ }^{5}$ University of Milan, "L. Sacco" Department of Biomedical and Clinical Sciences, Milan, Italy ${ }^{6}$ University of bologna, Alma Mater Research Institute for Humancentered Artificial Intelligence, Bologna, Italy

Keywords deep learning, magnetic resonance imaging, mild cognitive impairment, small vessel disease

\section{Purpose}

Mild cognitive impairment (MCI) is one of the consequences of small vessel disease (SVD) and the prediction of the cognitive status of patients with SVD and MCI from neuroimaging data is crucial to identify possible markers of the cognitive deficit. In recent years, deep learning schemes have been applied for the prediction of the cognitive status from brain imaging. Indeed, the ability to extract patterns and latent features from complex data, such as magnetic resonance imaging (MRI) data, makes deep learning schemes and, in particular, convolutional neural networks $(\mathrm{CNN})$, a powerful tool in neuroimaging applications. The objective of this study is to develop and test a 2D-CNN model to predict the cognitive status of patients with SVD and MCI in terms of the Trial Making Test part A (TMT-A) score-a popular neuropsychological test sensitive to psychomotor speed and executive functioningfrom multi-input data, i.e., MRI-derived features and demographic data.

\section{Methods}

In our study, we considered 58 patients with SVD and MCI from one center of the VMCI-Tuscany study (27 women and 31 men, aged $74.18 \pm 6.98$ years, mean \pm standard deviation) [1]. For each patient, MRI data were collected using different techniques. In 


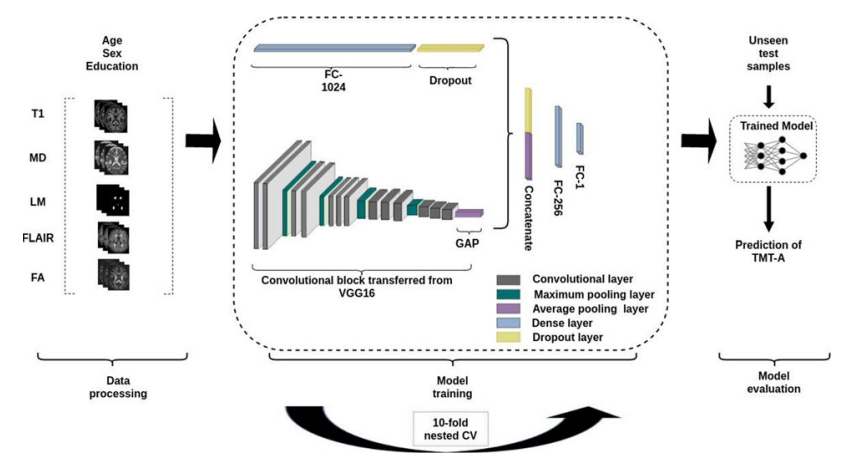

Fig. 1 Overview of the architecture of the proposed CNN for prediction of the TMT-A score

particular, T1-weighted, T2-weighted fluid attenuated inversion recovery (FLAIR), white matter hyperintensity lesion maps (LM), mean diffusivity (MD) and fractional anisotropy (FA) features derived from diffusion tensor imaging (DTI), resulting in five sets of voxel-wise MRI-derived feature maps, were available [1-2]. These maps were used, separately, as input to a $2 \mathrm{D}-\mathrm{CNN}$ after a linear and non-linear image registration onto the MNI152 standard template (Figure 1). Since our CNN has a 2D architecture, we sliced each volume into $2 \mathrm{D}$ images in the axial plane and, for each patient, we selected the 16 most informative slices according to the entropy maximization principle, i.e., the 16 slices that maximized the entropy value. Then, a voxel-wise standard feature scaling has been applied to achieve faster convergence.

Since the size of the training set was relatively small, we employed a transfer learning technique by adapting a pre-trained VGG16 model, trained on a large dataset of labeled natural images (ImageNet dataset). Then, we modified the VGG16 architecture to be able to feed multiple inputs (MRI-derived feature maps and demographic data, i.e., sex, age and education level of participants) (Figure 1). Specifically, while VGG16 is a single input CNN that accepts only a 2D image as input, our proposed model consisted of two input branches: a CNN and a fully connected (FC) branch. The $\mathrm{CNN}$ branch is formed by the convolutional layers of the VGG16 model and by a newly added global average pooling (GAP) layer that replaces the FC layers of the VGG16 network. This branch takes the MRI-derived feature map as input. On the other hand, the FC branch is constituted of a FC layer followed by a dropout layer and accepts demographic data as input. The outputs from the two branches are concatenated and further processed by the last FC layers.

For training and validation of the proposed model, we used a tenfold nested CV loop, where the inner fold was used for grid-search hyperparameter tuning of the model and the outer fold for evaluating the generalization ability of the optimized model on unseen test samples. The nested CV was based on a subject-level to assure that no data leakage was created during the data split. The adaptive Adam optimizer was chosen for training all models for 40 epochs with a mini-batch gradient descent algorithm and batch size of 128 .

To assess the model's performance, the average value of the Pearson's correlation coefficient between the actual and predicted values of the TMT-A score on the outer folds was computed.

Results

Table 1 illustrates an overview of the results. The model's development, training, validation and testing were implemented using Python code and Keras library (using a Tensorflow backend). A workstation equipped with Ubuntu 16.04, a high performance 12 GB G5X frame buffer NVIDIA TITAN X (Pascal) GPU with 3584 CUDA cores and a 64-bit 16 M RAM hardware was used. For each model, the computational time needed to run a nested CV loop was about $75 \mathrm{~h}$.
Table 1 Average Pearson's correlation coefficient between the actual and predicted values of the TMT-A score on the test sets of the outer folds (the bold value highlights the hest performance)

\begin{tabular}{|l|ccccc|}
\hline & \multicolumn{5}{c|}{ Feature type } \\
\cline { 2 - 6 } Cognitive test & T1 & FLAIR & LM & MD & FA \\
\hline TMT-A & 0,438 & 0,451 & 0,397 & 0,513 & 0,504 \\
\hline
\end{tabular}

\section{Conclusion}

In this study, the cognitive status of patients with SVD and MCI, measured by the TMT-A score, was predicted using CNN models trained on multiple MRI features. Although all the models employing different MRI-derived features produced good predictions, the TMTA score was best predicted using features derived from DTI data (MD and FA maps), yielding a correlation coefficient of 0.513 and 0.504 , respectively. These values were greater than that obtained in the same population using a least absolute shrinkage and selection operator (LASSO) regression trained on 13 demographic and neuroimaging features (coefficient of correlation $=0.354$ ) [1]. However, in the previous study, DTI-derived indices were not available and the model predicted demographically adjusted TMT-A scores, rather than raw scores. Our results are in accordance with the fact that cognitive deficits associated with information processing speed are mainly the effect of white matter damage which may be reflected by changes of DTI-derived indices [2]. In conclusion, our results showed that a deep learning approach could be a valuable tool in predicting the cognitive status of patients with SVD and MCI.

\section{References}

[1] Pantoni L, Marzi C, Poggesi A, Giorgio A, De Stefano N, Mascalchi M, Inzitari D, Salvadori E, Diciotti S (2019) Fractal dimension of cerebral white matter: A consistent feature for prediction of the cognitive performance in patients with small vessel disease and mild cognitive impairment. NeuroImage: Clinical, 24, p.101990

[2] Ciulli S, Citi L, Salvadori E, Valenti R, Poggesi A, Inzitari D, Mascalchi M, Toschi, N., Pantoni, L. and Diciotti, S., 2016. Prediction of impaired performance in trail making test in MCI patients with small vessel disease using DTI data. IEEE journal of biomedical and health informatics, 20(4), pp. 1026-1033.

\section{Machine learning for lung CT texture analysis in connective tissue disease: capability for disease severity and therapeutic effect evaluations}

Y. Ohno ${ }^{1,2,3}$, K. Aoyagi ${ }^{4}$, D. Takenaka ${ }^{5}$, T. Yoshikawa ${ }^{5,6}$, Y. Fujisawa $^{4}$, N. Sugihara ${ }^{4}$, H. Hattori ${ }^{1}$, K. Murayama ${ }^{1}$, H. Toyama $^{1}$ ${ }^{1}$ Fujita Health University School of Medicine, Radiology, Toyoake, Japan ${ }^{2}$ Fujita Health University School of Medicine, Joint Research Laboratory of Advanced Medical Imaging, Toyoake, Japan ${ }^{3}$ Kobe University Graduate School of Medicine, Division of Functional and Diagnostic Imaging Research, Department of Radiology, Kobe, Japan ${ }^{4}$ Canon Medical Systems Corporation, Otawara, Japan ${ }^{5}$ Hyogo Cancer Center, Diagnostic Radiology, Akashi, Japan ${ }^{6}$ Kobe University Graduate School of Mediciine, Division of Functional and Diagnostic Imaging Research, Department of Radiology, Kobe, Japan 
Keywords Computer-aided volumetry, Machine Learning, CT, Texture Analysis

\section{Purpose}

In response to a need for quantitative assessment of interstitial lung involvement, machine learning (ML)-based CT texture analysis was developed for patients with various pulmonary diseases. Recently, we developed and proposed ML-based CT texture analysis software and demonstrated its" capability to play as second reader similar to chest radiologists with more than 20 years experiences. In this situation, we hypothesized that our newly developed ML-based algorithm has a better potential than qualitatively assessed disease severity for disease severity assessment and treatment response evaluation for patients with connective tissue diseases. The purpose of this study was to evaluate the capability of ML-based CT texture analysis software for disease severity and treatment response assessments in comparison with qualitatively assessed thin-section CT for patients with connective tissue disease (CTD).

\section{Methods}

Between January 2016 and December 2017, 149 patients with CTD related ILD (CTD-ILD) who underwent initial and follow-up CTs, as well as pulmonary function and serum KL-6 level tests, were initially included as test cases with no overlaps between training, validation and test cases. 149 consecutive patients with CTD-ILD were included, consisting of 56 males ( $60 \pm 11$ years, age range: $39-83$ years) and 93 females ( $58 \pm 9$ years, age range: $30-85$ years) comprising 54 cases of progressive scleroderma (PSS), 34 of dermatomyositis (DM), 29 of rheumatoid arthritis (RA), 14 of polymyositis (PM), 7 of mixed connective tissue disease (MCTD), 7 of anti-neutrophil cytoplasmic antibody (ANCA)-associated vasculitis, and 4 of Sjogren's syndrome. Changes in disease severity between two serial CT examinations of each of these cases were assessed into three groups: Stable, $n=188$; Worse, $\mathrm{n}=98$; and Improved, $\mathrm{n}=78$. Next, quantitative index changes were determined by using software as well as qualitative disease severity scores. To determine the relationship among all radiological findings, each pulmonary function parameter or serum KL-6 level, step-wise regression analysis were performed. To evaluate differences in each quantitative index as well as in disease severity score between two serial CT examinations, Tukey"s honestly significant difference (HSD) test was performed to determine differences among the three statuses. Stepwise regression analyses were performed to determine changes in each pulmonary functional parameter and all quantitative indexes between two serial CTs.

\section{Results}

Results of stepwise regression analysis of differences between all quantitative radiological indexes and each pulmonary functional parameter as well as serum KL-6 level revealed that FEV1/FVC \% was significantly affected by $\%$ normal lung and $\%$ reticulation $\left(\mathrm{r}^{2}=0.27, p=0.04\right), \% \mathrm{VC}$ was significantly affected by $\%$ normal lung, \% reticulation and \% GGO $\left(\mathrm{r}^{2}=0.36, p=0.01\right)$, while \% $\mathrm{DL}_{\mathrm{CO}} / \mathrm{V}_{\mathrm{A}}$ was significantly affected by $\%$ normal lung, \% reticulation and \% GGO $\left(\mathrm{r}^{2}=0.27, p=0.04\right)$. In addition, serum KL-6 level was significantly affected by $\%$ normal lung and \% reticulation $\left(\mathrm{r}^{2}=0.27, p=0.04\right) . \Delta \%$ normal lung, $\Delta \%$ consolidation, $\Delta \%$ GGO, $\Delta \%$ reticulation and $\Delta$ disease severity score showed significant difference among the three statuses $(p<0.05) . \Delta \%$ honeycomb showed a significant difference between the "Stable" and "Worse" groups and between the "Worse" and "Improved" groups $(p<0.01)$. All differences in pulmonary functional parameters as well as in serum KL-6 levels were significantly affected by $\Delta \%$ normal lung, $\Delta \%$ reticulation and $\mathrm{D} \%$ honeycomb $\left(0.16 \leq \mathrm{r}^{2} \leq 0.42, p<0.05\right)$. Conclusion

Newly developed ML-base CT texture analysis has better potential than qualitatively assessed thin-section CT for disease severity assessment and treatment response evaluation for patients with connective tissue diseases.

\section{Computer-aided prediction of COVID-19 progression using unsupervised deep learning of chest CT images}

T. Uemura ${ }^{1,2}$, J. Näppi $^{1}$, C. Watari ${ }^{1}$, T. Hironaka ${ }^{1}$, T. Kamiya ${ }^{2}, \mathrm{H}$. Yoshida $^{1}$

${ }^{1}$ Massachusetts General Hospital/Harvard Medical School, Imaging, Boston, United States ${ }^{2}$ Kyushu Institute of Technology, Kitakyushu, Japan

Keywords computer-aided prediction, deep learning, survival analysis, COVID-19

\section{Purpose}

The severity of the cases with COVID-19 together with its rapid progression has placed great pressures on healthcare services around the world. Therefore, fast and accurate prediction of the disease progression of patients with COVID-19 is needed for logistical planning of the healthcare resources. The purpose of this study was to develop a prediction model, called pix2surv, for predicting the COVID-19 progression from patients" chest CT images.

\section{Methods}

\section{Subjects and chest CT}

Patients who had been diagnosed as COVID-19 positive based on a positive result for SARS-COV-2 by the reverse transcriptase-polymerase chain reaction (RT-PCR) test were included retrospectively in this study. As a result, we identified, from the medical records of our institution, high-resolution chest CT images of 105 patients with confirmed COVID-19. For these patients, the CT scans were performed with a single-phase, low-dose acquisition protocol by use of multi-channel CT scanners with slice thickness of $0.625-1.5 \mathrm{~mm}$, pitch of $0.3-1.6$, tube voltage of $80-140 \mathrm{kVp}$, and automatic tube current modulation.

\section{pix2surv model}

The pix2surv is based on an adversarial time-to-event model [1], which was adapted to predict a patient"s disease progression. Here, we define the survival time of a patient as the number of days from the patient"s chest CT scan to either ICU admission or death. Figure 1 shows our generative-adversarial-network-based architecture of the pix 2surv model, in which the time generator $G$ is used to generate or estimate a "survival-time image" from the input CT images of a patient. The survival-time image is an image that has a single survival time value at each pixel. The discriminator $D$ attempts to differentiate the "estimated pair" of an input CT image and a generated survival-time image, from the "observed pair" of an input CT image and the observed true survival-time image of the patient. The training of pix2surv involves the optimization of $G$ and

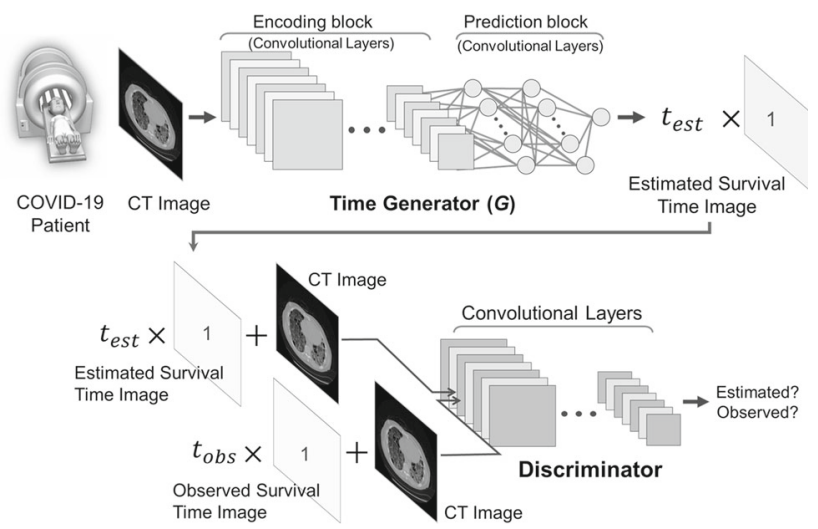

Fig. 1 Schematic architecture of the proposed pix2surv model for the prediction of the COVID-19 progression 
(a) CPC

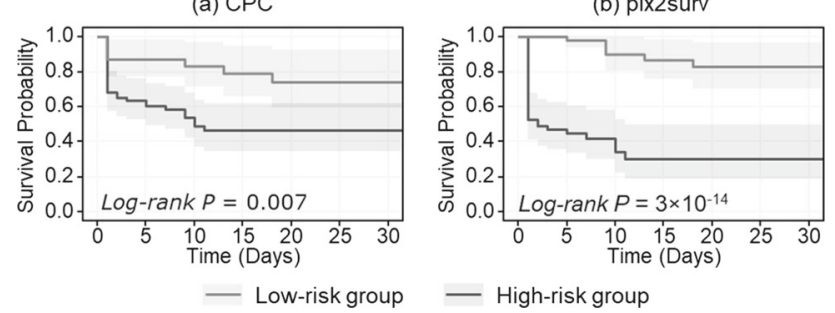

Fig. 2 Kaplan-Meier survival curves of the COVID-19 patients stratified into a low-risk patient group (gray color) and a high-risk patient group (black color) based on a the CPC and $\mathbf{b}$ pix 2surv. The shaded areas represent the $95 \%$ confidence intervals of the survival curves

$D$ through a modified min-max objective function, so that $G$ learns to generate a survival time (predicted disease progression) that is close or equal to the observed survival time (observed disease progression). Total severity score for crazy paving and consolidation (CPC) We evaluated the performance of pix2surv in the prediction of COVID-19 progression in comparison with an existing COVID-19 progression predictor of the "total severity score for crazy paving and consolidation" (CPC) [2]. The CPC for a patient was assessed by a pulmonologist with over 20-year experience as the sum extent of crazy paving and consolidation in the chest CT images of the patient based on the "total severity score" criteria, where the sum involvement of the five lung lobes is taken as the total lung score (value range: 0-20).

\section{Evaluation}

As metrics of the prediction performance, we used the concordance index (C-index), as well as the relative absolute error (RAE) that is defined by $\Sigma_{\mathrm{i}}\left|t_{\mathrm{i}}^{\text {est }}-t_{\mathrm{i}}\right| / t_{\mathrm{i}}$, where $t_{\mathrm{i}}^{\text {est }}$ is the predicted survival time and $t_{\mathrm{i}}$ is the observed survival time for patient $i$. A patient-based bootstrap method with 100 replications was used to obtain an unbiased estimate of the C-index and RAE, and they were compared with those of the CPC using a two-sided unpaired $t$ test.

We also evaluated the stratification of the patients into low- and high-risk groups by generating Kaplan-Meier survival curves based on the predicted survival times. The log-rank test was used to evaluate the difference between the survival curves of the risk groups.

\section{Results}

Our experimental results show that the C-index values (larger is better) obtained from the CPC and pix2surv were $62.1 \%$ [95\% confidence interval (CI): 61.3, 63.0] and 82.5\% [95\% CI: 81.5, 83.5], respectively. The RAE values (smaller is better) obtained from the CPC and pix2surv were $49.9 \%$ [95\% CI: $49.3,50.5$ ] and $26.3 \%$ [95\% CI: 25.5, 27.0], respectively. These results indicate that the prediction performance of pix2surv is significantly higher than that of the CPC $(p<0.0001)$, and that its prediction error is significantly lower than that of the CPC $(p<0.0001)$.

Figure 2 shows the Kaplan-Meier survival curves of the COVID19 patients stratified into low- and high-risk patient groups based on the survival times predicted by (a) the $\mathrm{CPC}$ and (b) pix2surv. Both visual assessment and the $P$-values from the log-rank test indicate that the separation between the two curves is larger with pix2surv than with CPC, indicating that pix2surv is more effective than CPC in the stratification of the progression risk of COVID-19 patients.

Conclusion

We developed a novel survival prediction model, pix2surv, which directly predicts disease progression from patient"s chest CT images. We demonstrated that pix2surv outperforms the CPC in predicting the disease progression in patients with COVID-19, indicating that pix2surv can be an effective predictor of the COVID-19 progression.

\section{References}

[1] Chapfuwa P, Tao C, Li C, Page C, Goldstein B, Carin L, Henao $\mathrm{R}$ (2018) Adversarial time-to-event modeling. Proceedings of the 35th International Conference on Machine Learning 2018, 734-743.

[2] Lyu P, Liu X, Zhang R, Shi L, Gao J (2020) The performance of chest CT in evaluating the clinical severity of COVID-19 pneumonia: identifying critical cases based on CT characteristics. Investigative Radiology 55:412-421.

\section{Leukoplakia lesion classification in Larynx contact endoscopy—narrow band imaging: preliminary results of a manual versus an automatic approach}

N. Esmaeili ${ }^{1,2}$ A. Illanes ${ }^{1}$, A. Boese ${ }^{1}$, N. Davaris ${ }^{3}$, C. Arens ${ }^{3}$, N. $\mathrm{Navab}^{2}$, M. Friebe ${ }^{1,4}$

${ }^{1}$ Otto-von-Guericke University, Medical Faculty, INKA-Innovation Laboratory for Image Guided Therapy, Magdeburg, Germany

${ }^{2}$ Technical University Munich, Chair for Computer Aided Medical Procedures and Augmented Reality, Munich, Germany ${ }^{3}$ Magdeburg University Hospital, Department of Otorhinolaryngology, Head and Neck Surgery, Magdeburg, Germany ${ }^{4}$ IDTM GmbH,

Recklinghausen, Germany

Keywords Leukoplakia, Contact Endoscopy, Narrow Band Imaging, Classification

\section{Purpose}

Laryngeal leukoplakia is a clinical term, describing the presence of a white plaque of the vocal fold. Laryngeal lesions with benign leukoplakia can remain stable for months or years; however, some of these lesions will eventually undergo malignant transformation. Contact Endoscopy (CE) in combination with Narrow-Band Imaging (NBI) - as a minimally invasive optical diagnostic imaging procedure - can improve the evaluation of these types of lesions by visualizing the microvascular changes in the area surrounding the leukoplakia [1]. In clinical practice, diagnosis of benign and malignant leukoplakia lesions based on the vascular patterns and other endoscopic features is a subjective process resulting in invasive surgical biopsy and subsequent histological examination. The main objective of this work is to evaluate the potential of an automatic machine learning-based approach for the classification of CE-NBI images of vascular patterns around leukoplakia into benign and malignant; and then, to compare the results with the multi-observer manual classification.

\section{Methods}

Video scenes of 40 patients with histologically examined leukoplakia lesions were acquired. An Evis Exera III Video System with a xenon light source plus integrated NBI-filter (Olympus Medical Systems, Hamburg, Germany) and a rigid 30-degree contact endoscope (Karl Storz, Tuttlingen, Germany) was used. 1998 high-resolution CE-NBI images with unique vascular patterns were manually extracted from these videos. The images were labeled into benign and malignant groups based on histological diagnosis according to the World Health Organization (WHO) classification.

Two image subsets were created from the CE-NBI dataset. The Subset I included a series of four to five randomly selected CE-NBI images of each patient with a total of 199 images. Subset II included 1799 images and was used as the training set in the automatic approach. The Subset I was evaluated by the otolaryngologists in the manual approach and then used as the testing set for the automatic approach. 
In the manual approach, two experienced otolaryngologists visually evaluated the CE-NBI images of Subset I. They were blinded to the histologic diagnosis and independently classified the lesions into benign and malignant based on the vascular patterns. Malignant labeled lesions with malignant histopathology were considered true positives for the calculation of sensitivity and specificity.

The method presented in [2] was used to perform the automatic classification. The algorithm has been tested on several CE-NBI datasets for the classification of laryngeal lesions and histopathologies. It consists of a pre-processing step involving vessel enhancement and segmentation. A feature extraction step was then applied to extract 24 geometrical features based on the consistency of gradient direction and the curvature level. The supervised classification step was conducted using the Polynomial Support Vector Machine (SVM) and k-Nearest Neighbor (kNN). Subset II was used for the hyperparameter tuning process with a grid search method and fivefold cross-validation. In the automatic approach, Subset I and Subset II were used as the testing and training sets, respectively. Each classifier was trained using the images" labels and feature vectors of the CE-NBI images of the training set. For the testing, the feature vectors computed from the CE-NBI images of the testing set were fed into the predictive model of each classifier, and then the expected labels were collected. This training and testing set strategy was used to be able to compare the results of manual and automatic classifications. The sensitivity and specificity were calculated from a confusion matrix for each classification.

\section{Results}

Table 1 presents the results of the automatic and manual classification in CE-NBI images of leukoplakia lesions. The mean specificity of 0.875 in manual classification can be explained by the difficulty to visually distinguish benign leukoplakia lesions from malignant ones based on the vascular pattern around the lesion. This issue happens due to the presence of similar microvascular structures in some benign and malignant leukoplakia lesions. Figure 1 illustrates the qualitative performance of the automatic approach. Two presented indicators (Histogram of Gradient Direction and Curvature) show different behaviors on CE-NBI images of benign and malignant leukoplakia lesions. Furthermore, the automatic classification showed a better performance than manual classification with a sensitivity of 1 by both classifiers. The sensitivity value of 1 means that all the CENBI images of malignant leukoplakia lesions were correctly classified by the predictive model of two classifiers. These results emphasize the potential of the automatic approach to solving the problem of misclassification of laryngeal leukoplakia lesions based on the evaluation of vascular patterns around the lesion.

\section{Conclusion}

The results showed that the visual differentiation of vascular patterns that indicate benign leukoplakia lesions from malignant ones can be challenging for otolaryngologists in clinical practice. The automatic algorithm demonstrated its ability in the classification of CE-NBI images of leukoplakia lesions. We believe that the combination of the geometrical features with machine learning classifiers has the potential to provide a confident way for clinicians to make the final

Table 1 Classification results of manual versus automatic approach for CE-NBI images

\begin{tabular}{llll}
\hline & Manual classification & \multicolumn{2}{l}{ Automatic classification } \\
\cline { 3 - 4 } & & SVM & kNN \\
\hline Sensitivity & 0.958 & 1 & 1 \\
Specificity & 0.875 & 0.872 & 0.897
\end{tabular}

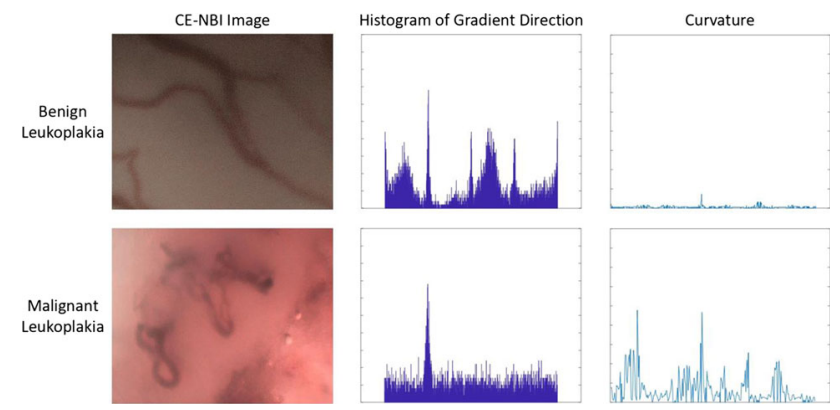

Fig. 1 Behavior of two indicators for CE-NBI images of vascular patterns around benign and malignant leukoplakia lesions

decision about the dignity of the laryngeal leukoplakia and prevent unnecessary surgical biopsies.

\section{References}

[1] Ni X.G, Zhu J.Q, Zhang Q.Q, Zhang B.G, Wang G.Q (2019). Diagnosis of Vocal Cord Leukoplakia: The Role of a Novel Narrow Band Imaging Endoscopic Classification. The Laryngoscope 129(2): 429-434.

[2] Esmaeili N, Illanes A, Boese A, Davaris N, Arens C, Friebe M (2019). Novel Automated Vessel Pattern Characterization of Larynx Contact Endoscopic Video Images. International Journal of Computer Assisted Radiology and Surgery 14(10): $1751-1761$

\section{Experimental evaluation of loss functions in YOLO-v3 training for the perforation detection and localization in colonoscopic videos}

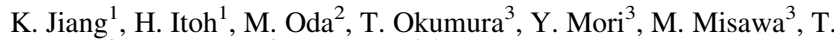
Hayashi $^{3}$, S. E. Kudo ${ }^{3}$, K. Mori ${ }^{1,4,5}$

${ }^{1}$ Nagoya University, Graduate School of Informatics, Nagoya, Japan

${ }^{2}$ Nagoya University, Information Strategy Office, Information and Communications, Nagoya, Japan ${ }^{3}$ Showa University Northern Yokohama Hospital, Digestive Disease Center, Yokohama, Japan ${ }^{4}$ Nagoya University, Information Technology Center, Nagoya, Japan

${ }^{5}$ National Institute of Informatics, Research Center for Medical

Bigdata, Tokyo, Japan

Keywords Deep learning, YOLO-v3, Colonoscopic video, Colonperform detection

\section{Purpose}

Endoscopic Submucosal Dissection (ESD) surgery [1] is a minimally invasive treatment for early gastric cancer. In ESD, physicians directly remove the mucosa around the lesion under internal endoscopy by using flush knives. However, the flush knife may accidentally pierce the colonic wall and generate a perforation on it. If there is a perforation, a patient needs emergency open surgery, since perforation can easily cause peritonitis. Our research purpose is to construct a Computer-Aided Diagnosis (CAD) system to support ESD surgery [1]. To support physicians in ESD surgery, the final goal is the prevention of perforations in ESD. At the current stage, image-based perforation detection is very challenging. Thus, we tackle with detection and localization of perforations in colonoscopic videos. We believe the automatic perforation-detection function is useful for the analysis of ESD videos for the development of a CAD system. 
In our previous research, we have used You Only Look Once (YOLO-v3) to detect and localize perforations [2]. Our experimental results indicated a trained YOLO-v3 can perform high perforation detection and localization accuracy. However, the sensitivity of detection results always stays at a low level. The low sensitivity problem illustrates that overlooking of perforation happed by a trained YOLO-v3. Purpose of this work is development of new loss functions for the training of YOLO-v3 for perforations detection and localization with high detection sensitivity.

\section{Methods}

In our previous work, we have constructed a perforation detection and localization model by training an original YOLO-v3 with our dataset [2]. For each training image, YOLO-v3 predicts a probability and a region for every detected perforation, as detection and localization tasks, respectively. YOLO-v3 predicts Intersection over Union (IoU) between a predicted region and a ground truth region. By multiplicate an estimated probability and IoU, YOLO-v3 outputs a score for each box. For the training of YOLO-v3, the original loss function of YOLOv3 consists of object and box losses. These two losses evaluate mean square error (MSE) between true class labels and scores, and error of location of bounding boxes between prediction and true boxes.

To improve detection sensitivity, we introduce two object losses. One is the cross-entropy (CE) loss, which evaluates the cross-entropy error between scores and true class labels. The other is the softmax loss, which evaluates the cross-entropy error between the true class labels and normalized scores, where we input scores to a softmax function. By combining one of the CE and the softmax losses with the original box loss, we have two loss functions for YOLO-v3 training. To compare with the previous method, we use the original object and box losses as loss function to train a YOLO-v3, too. We experimentally evaluate the detection and localization performances of the proposed methods with several evaluation terms.

\section{Results}

We used 1814 and 385 images for model training and validation, respectively. For evaluation of the detection and localization performance, we collected 22,598 and 150 images, respectively. All detail settings of the constructed model are the same as the original YOLOv3. For the training process, we set the mini-batch size to be 64 to train all models for 300 epochs on NVIDIA Tesla V100 PCIe 32 GB with CUDA 10.0 and used Adam as an optimizer function.

For detection performance evaluation, we introduced the accuracy, recall, and Receiver Operating Characteristic (ROC) curve. Figure 1 shows the ROC curve of three trained models, respectively. For localization performance evaluation, we introduced the mean Average Precision (mAP) score. We set the localization thresholds to be 0.6. We defined a true positive box when the IoU of the box is larger than 0.6. Table 1 shows accuracy, recall, Area Under Curve (AUC) score, and $\mathrm{mAP}$ score of three trained models. Table 1 illustrates the proposed method did not provide a higher mAP score than the original YOLO-v3. This phenomenon is due to the loss function is the combination of box and object losses, change of object loss also affects the backpropagation of the localization part.

\section{Conclusion}

This work experimentally evaluated two loss functions in YOLO-v3 for the training of perforation detection and localization in colonoscopic videos. The constructed model trained by the box loss plus CE loss achieved 0.881 accuracy, 0.631 recall, 0.863 AUC, and 0.732 mAP in detection and localization for the limited-size samples. Compare with the MSE and softmax losses, the CE loss has larger output when misclassification happened, which is better for optimization of the detection part in YOLO-v3. To acquire higher localization accuracy, we will try to modify weights of two losses in the loss function and looking forward to implying new loss functions for YOLO-v3 in the future. Parts of this research were supported by MEXT/JSPS KAKENHI (26108006, 17H00867, 17K20099), the

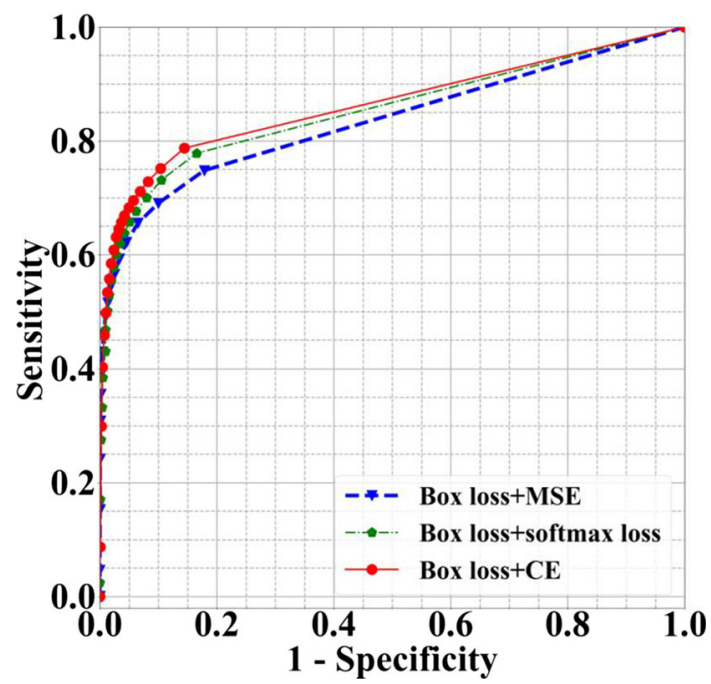

Fig. 1 ROC curve of three trained models

Table 1 Qualitative evaluation of detection and localization performances

\begin{tabular}{lllll}
\hline Loss functions & Accuracy & Recall & AUC & mAP \\
\hline Box loss + MSE & 0.835 & 0.458 & 0.834 & 0.746 \\
Box loss + softmax loss & 0.866 & 0.555 & 0.853 & 0.698 \\
Box loss + CE & 0.881 & 0.631 & 0.863 & 0.732 \\
\hline
\end{tabular}

JSPS Bilateral International Collaboration Grants, the AMED (19hs0110006h0003), and the Hori Sciences \& Arts Foundation.

\section{References}

[1] Saito Y, Uraoka T, Yamaguchi Y, Hotta k, Sakamoto N, Ikematsu H, Fukuzawa M, Kobayashi N, Nasu J, Michida T, Yoshida S, Ikehara H, Otake Y, Nakajima T, Matsuda T, Saito D (2010) A prospective, multicenter study of 1111 colorectal endoscopic submucosal dissections (with video), Gastrointestinal Endoscopy 2010, 72(6):1217-1225

[2] Kai J, Itoh H, Oda M, Okumura Y, Mori Y, Misawa M, Hayashi T, Kudo S, Mori K (2020) Preliminary Study of Perforation Detection and Localization for Colonoscopy Video. Proceedings of JAMIT Annual Meeting 2020, 2020:142-147

\section{New computer-aided detection schemes to detect breast cancer in two-dimensional synthesized mammograms and full-field digital mammograms}

M. Tan ${ }^{1,2}$, M. Al-Shabi ${ }^{1}$, W. Y. Chan ${ }^{3}$, L. Thomas ${ }^{3}$, K. H. $\mathrm{Ng}^{3}$ ${ }^{1}$ Monash University Malaysia, Electrical and Computer Systems Engineering Discipline, School of Engineering, Subang Jaya, Selangor, Malaysia ${ }^{2}$ University of Oklahoma, School of Electrical and Computer Engineering, Norman, United States ${ }^{3}$ University of Malaya, Department of Biomedical Imaging and University of Malaya Research Imaging Centre, Faculty of Medicine, Kuala Lumpur, Malaysia

Keywords Mammography, Synthesized mammography, Structural similarity, Breast density 


\section{Purpose}

Breast cancer screening with two-dimensional (2D) full-field digital mammography (FFDM) is the imaging modality that is the gold standard currently used in many countries to reduce cancer mortality in women. In 2011, digital breast tomosynthesis (DBT) was approved by the FDA to be used with FFDM to screen women for breast cancer. However, this leads to double radiation exposure in women.

Thus, Hologic Inc. obtained FDA approval to produce 2D synthesized mammograms (2DSMs) from DBT data, called "C-view" images. If 2DSM images can be used in place of FFDM in standard screening procedure, this eliminates the need for exposing women to double radiation exposure.

However, the question remains whether 2DSMs are equivalent in diagnostic quality to replace FFDMs. Thus, many studies were conducted to examine the use of 2DSM in conjunction with DBT over conventional FFDM images, but almost all of them were subjective observer studies [1].

This study compares the diagnostic quality of 2DSMs with FFDMs using a different objective based approach by computing structural similarity measures and feature correlations between the two modalities. We also develop and compare two new computeraided detection (CAD) schemes based on global features computed on 2DSM and FFDM, which to the best of our knowledge is the first study of its kind.

\section{Methods}

We studied 407 women subjected to 2D FFDM and DBT examination for screening or diagnostic purposes at our institution from September 2014 to February 2016. Thus, 3216 craniocaudal (CC) and mediolateral oblique (MLO) mammograms were analyzed altogether.

We calculated 152 structural similarity, texture and mammographic density based features previously used to predict breast cancer risk [2] on all mammograms. We included/computed boxplots to analyze the interquartile ranges/variations of the structural similarity features from their median values.

To compare the performance of 2DSM and FFDM for developing two cancer detection schemes based on global features computed on the whole breast region, we computed 112 texture and mammographic density features, and trained and examined different classifiers on them.

We compared the performances of the linear discriminant analysis (LDA), logistic regression, and bagged decision trees classifiers. To prevent "overfitting" of the classifiers on the high-dimensionality features, we applied stepwise regression feature selection to select the "relevant" features in a leave-one-case-out (LOCO) cross-validation scheme.

To compare the performances of the two global detection models, we computed the area under a receiver operating characteristic (ROC) curve (AUC). Using all classifier-generated detection scores, we computed AUC and $95 \%$ confidence intervals (CIs) using a maximum likelihood data analysis based ROC curve fitting program (ROCKIT http://www.radiology.uchicago.edu/krl/). We also evaluated statistically significant differences at the 5\% significance level using DeLong"s test to compare the classifier performances between 2DSM and FFDM.

\section{Results}

Figure 1 displays the boxplots of the highest structural similarity features computed in our study. It can be observed that the structural similarity feature of the CC image had the highest median, namely CB-CW-SSIM of the Weber Local Descriptor (WLD) differential excitation feature computed at scale $s=24$. The CC image had the highest median, namely 0.92 for CC1; MLO1 without the pectoral muscle had a very low median, namely 0.797 . MLOpect1 with the pectoral muscle, had a higher median, namely 0.90 , but it was still less than $\mathrm{CC} 1$. The results show that $\mathrm{CC}$ images were the most similar

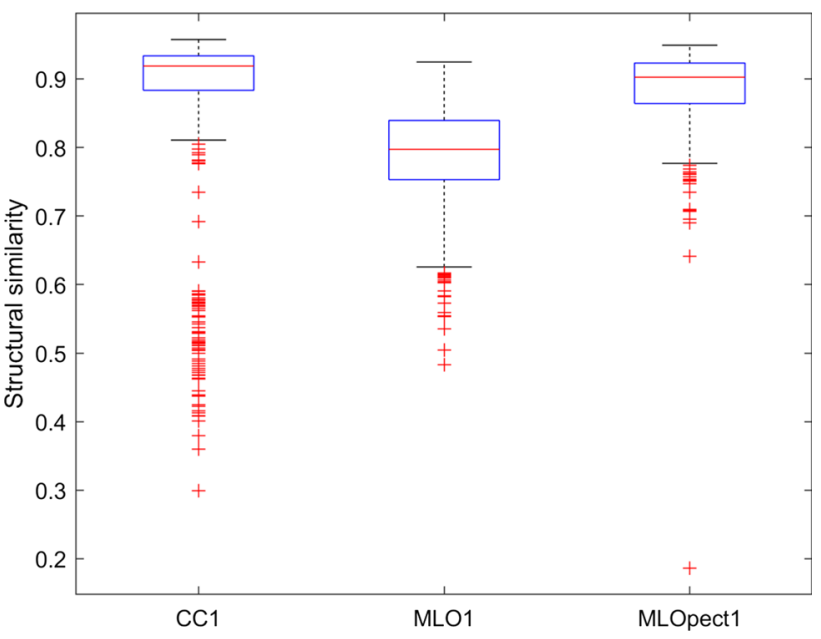

Fig. 1 Boxplots of the highest structural similarity feature values computed in our study. CC1, MLO1 and MLOpect1 correspond to CB-CW-SSIM WLD differential excitation $(s=24)$ computed on the $\mathrm{CC}, \mathrm{MLO}$ without pectoral muscle, and MLO with pectoral muscle view images, respectively

Table 1 Computed AUC values and corresponding 95\% CIs when applying the classifiers to FFDM and 2DSM image sets using a LOCO cross-validation method

\begin{tabular}{|c|c|c|c|c|}
\hline $\begin{array}{l}\text { Image } \\
\text { type }\end{array}$ & Classifier & AUC (SE) & $95 \% \mathrm{CI}$ & $\begin{array}{l}P \text {-value computed } \\
\text { from DeLong"s } \\
\text { test }\end{array}$ \\
\hline 2DSM & $\begin{array}{l}\text { Bagged } \\
\quad \text { decision trees }\end{array}$ & $0.878(0.034)$ & [0.799-0.933] & 0.103 \\
\hline FFDM & $\begin{array}{l}\text { Bagged } \\
\quad \text { decision trees }\end{array}$ & $0.756(0.052)$ & [0.643-0.846] & \\
\hline 2DSM & LDA & $0.737(0.059)$ & [0.611-0.838] & 0.382 \\
\hline FFDM & LDA & $0.710(0.058)$ & {$[0.587-0.813]$} & \\
\hline 2DSM & $\begin{array}{l}\text { Logistic } \\
\quad \text { regression }\end{array}$ & $0.789(0.045)$ & {$[0.689-0.866]$} & 0.265 \\
\hline FFDM & $\begin{array}{l}\text { Logistic } \\
\quad \text { regression }\end{array}$ & $0.721(0.057)$ & {$[0.600-0.821]$} & \\
\hline
\end{tabular}

between 2DSM and FFDM modalities and coarse structures computed at a coarse scale (i.e., $s=24$ ) are similar between both modalities.

Table 1 tabulates the AUC values and 95\% CIs of all 3 classifiers that we analyzed in the LOCO cross-validation scheme. We observe that all 3 classifiers trained on 2DSM outperformed the corresponding classifiers trained on FFDM. The best-performing classifier was bagged decision trees. The AUC values of all corresponding classifiers achieved for FFDM and 2DSM were not significantly different using DeLong"s test at the 5\% significance level.

\section{Conclusion}

This study presents a comprehensive objective evaluation of similarity between 2DSM and FFDM images, with results indicating that coarse structures are similar between the two images. The performance of global mammographic image feature-based cancer detection schemes trained on 2DSM images outperformed corresponding schemes trained on FFDM images. Further investigation is required to examine whether DBT can replace FFDM as a standalone technique, negating the need for women to undergo mammography twice, especially for the development of automated objective-based methods. 


\section{References}

[1] Hofvind S, Hovda T, Holen AS, Lee CI, Albertsen J, Bjorndal H, Brandal SHB, Gullien R, Lomo J, Park D, Romundstad L, Suhrke P, Vigeland E, Skaane P (2018) Digital Breast Tomosynthesis and Synthetic 2D Mammography versus Digital Mammography: Evaluation in a Population-based Screening Program. Radiology 287 (3):787-794. https://doi.org/10.1148/ radiol.2018171361

[2] Tan M, Zheng B, Leader JK, Gur D (2016) Association between Changes in Mammographic Image Features and Risk for Nearterm Breast Cancer Development. IEEE Trans Med Imaging 35 (7):1719-1728

\section{$\overline{\text { Malignant tumor detection on screening breast MRI }}$ with deep learning}

\author{
Z. L. Tse ${ }^{1}$, D. R. Chen $^{2}$, H. K. Wu ${ }^{3}$, Y. L. Huang ${ }^{1}$ \\ ${ }^{1}$ Tunghai University, Department of Computer Science, Taichung, \\ Taiwan ${ }^{2}$ Changhua Christian Hospital, Comprehensive Breast Cancer \\ Center, Changhua, Taiwan ${ }^{3}$ Changhua Christian Hospital, \\ Department of Medical Imaging, Changhua, Taiwan
}

Keywords Breast cancer, Deep learning, Breast magnetic resonance image, Malignant tumor detection

\section{Purpose}

Breast magnetic resonance imaging (MRI) has many advantages such as non-invasive, no radiation damage and multi-view section. Breast MRI is often used in women who already have been diagnosed with breast cancer, to help measure the size of the cancer and look for other tumors in the breast. MRI uses radio waves and strong magnets to make detailed three-dimensional (3D) images of the breast tissue after contrast agent injection. Breast MRI offers valuable information about many breast conditions which are unable to identify by other imaging modalities [1]. Hence, MRI performs to monitor the response of the neo-adjuvant chemotherapy which is the administration of therapeutic agents prior to surgery. The shape and contour of the malignant tumor would be used as a significant information to evaluate the effect of the neo-adjuvant chemotherapy or breast-conserving surgery. The aim of this study is to develop an accurate tumor detection/screening scheme on 3D breast MRI by using deep learning techniques. The detection procedure identified the malignant tumor region using region-based convolutional neural networks (R-CNN) and image processing techniques.

\section{Methods}

\section{- Data acquisition}

This study utilized the breast MRI image database from 30 patients. MRI was performed with the patient in the prone position. Examinations were performed with a 3.0-T commercially available system (Verio ${ }^{\circledR}$; Siemens AG, Erlangen, Germany) and use of a dedicated16 Channel breast coil. Imaging sequences included a localizing sequence, an axial tse_T1 weighted $(3 \mathrm{~mm})$, tse_T2_tirm, pre, during, and post-Gd 3D-FSPGR $(1 \mathrm{~mm})$ with fat saturation images, before and five times after rapid bolus injection of $0.1 \mathrm{mmol} / \mathrm{L}$ gadobenate dimeglumine (Multihence ${ }^{\circledR}$; Bracco, s.p.a., Milano, Italy) per kilogram of body weight at a rate of $2 \mathrm{ml} / \mathrm{s}$; followed by a saline flush, acquired at $60 \mathrm{~s}$ intervals were obtained. All obtained images were stored on the hard disk and transferred to a personal computer using a DICOM (Digital Imaging and Communications in Medicine) connection for image analysis.

- Malignant tumor detection with deep-learning

Mask R-CNN is a two-stage framework [2]: the first stage scans the image output feature map and output through region proposal network (RPN) generates proposals; the second stage classifies proposals and generates bounding boxes and masks. In this study, the mask R-CNN model utilized the ResNet (deep residual network) and FPN (feature pyramid networks) backbone with standard convolution and fully connected heads for mask and box prediction, respectively. This model would obtain the best speed/ accuracy trade-off. Generally speaking, we believe that shallower features mainly provide detailed information, while deeper information provides semantic information. The deep-learning techniques have the opportunity as a robust automatic scheme for identifying malignant tumors on breast MRI without human intervention.

In the proposed method, the mask R-CNN model identified the region of malignant tumors from transverse, coronal and sagittal planes. The $3 \mathrm{D}$ regions generated from the transverse, coronal and sagittal planes denoted RT, RC and RS, respectively. Then, this study developed the intersection version of the $3 \mathrm{D}$ regions from the three views. RAND denoted the contour of region that occurred simultaneously (such as AND operation) from RT, RC and RS. RAND was performed as the final result of malignant tumor regions.

Results

This study totally used 30 breast MRI with malignant tumors (pathology-proven cases) to test the accuracy of the proposed method. The k-fold cross-validation method was used to estimate the performance. The $k$ is selected as 3 in the simulations and each group has 10 breast MRI. The sensitivity of the proposed detection method for malignant tumors is $100.0 \%$ and the positive predictive value (PPV) is $71.0 \%$. Figures 1 illustrates the identified results of RT, RC and RS from a sample case. The 3D view of the final result RAND is shown in Fig. 2. Moreover, the proposed method identified tumors from the three $2 \mathrm{D}$ planes which could save much of the time required to detect/ screen malignant tumor on 3D breast MRI.

\section{Conclusion}

This study presented an efficient method for automatically detecting malignant tumors in breast MRI. The proposed method applied the deep-learning model to automatic produce the region of malignant tumors from transverse, coronal and sagittal planes. The experimental results revealed that the proposed method can practically identify malignant tumor from breast MRI images. Results of this study could be utilized to $3 \mathrm{D}$ contouring procedure for malignant tumor and then expected to be helpful for surgeon in evaluating the effect of neoadjuvant chemotherapy or breast-conserving surgery.

Acknowledgements

This work was supported by the Ministry of Science and Technology, Taiwan, Republic of China, under Grant MOST 109-2221-E-029-024.
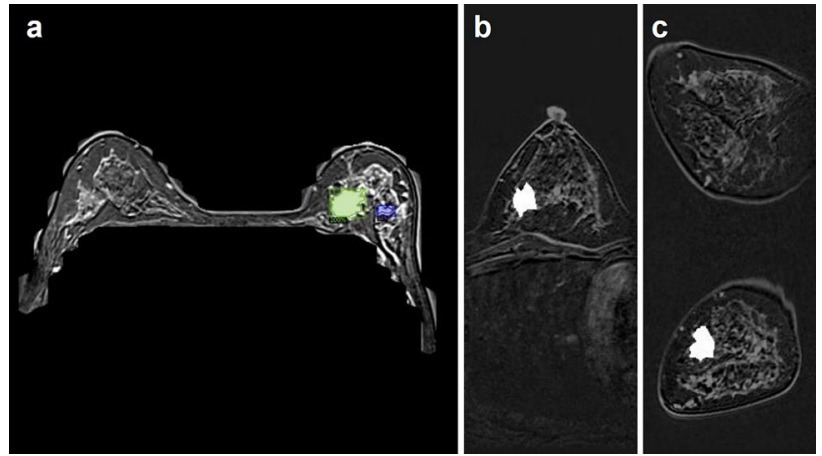

Fig. 1 The 3D regions obtained from a the transverse, $\mathbf{b}$ the sagittal and $\mathbf{c}$ the coronal planes 

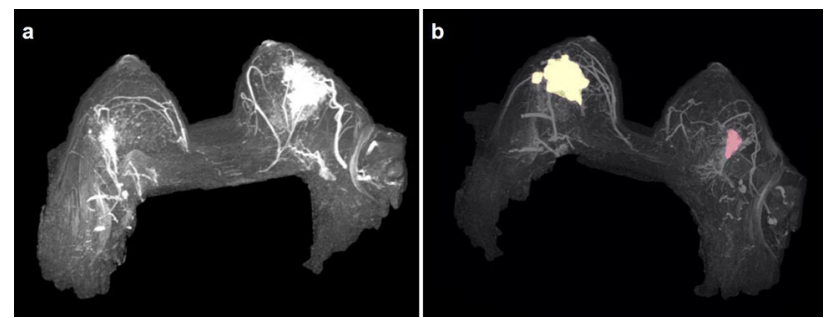

Fig. 2 3D view of a original breast MRI and $\mathbf{b}$ the final identified result

\section{References}

[1] Cho N, Im SA, Park IA, Lee KH, Li M, Han W, Noh DY, Moon WK (2014) Breast cancer: early prediction of response to neoadjuvant chemotherapy using parametric response maps for MR imaging. Radiology, vol. 272, no. 2, pp. 385-3966.

[2] He K, Gkioxari G, Dollar P, Girshick R (2020) Mask R-CNN. IEEE Trans Pattern Anal Mach Intell, vol. 42, no. 2, pp. 386-397.

\section{$\overline{\text { Bayesian approach for hotspot extraction from a bone }}$ scintigram}

M. Hara $^{1}$, A. Saito ${ }^{1}$, J. Kawabe ${ }^{2}$, S. Higashiyama ${ }^{2}$, H. Daisaki ${ }^{3}$, A. Shimizu ${ }^{1}$

${ }^{1}$ Tokyo University of Agriculture and Technology, Institute of Engineering, Koganei, Tokyo, Japan ${ }^{2}$ Osaka City University, Nuclear Medicine, Osaka, Japan ${ }^{3}$ Gunma prefectural college of health sciences, Nuclear Medicine, Maebashi, Japan

Keywords Bone scintigram, Deep learning, Hotspots, Probabilistic atlas

\section{Purpose}

Bone scintigraphy is useful for the diagnosis of bone metastases in prostate or breast cancers. The bone scan index (BSI) is a popular index for quantitatively estimating the stage of bone metastasis that requires bone segmentation and hotspot extraction. Accurate extraction of hotspots caused by bone metastatic lesions is a difficult task because of the high similarity between hotspots of bone metastasis and hotspots of non-malignant lesions, such as physiological accumulation.

Computer-aided diagnosis systems [1,2] have been developed to compute the BSI. These systems, however, often under-extract a part of the bone metastatic lesion or over-extract a part of non-malignant lesions, which are often observed at specific locations over multiple cases. Prior knowledge of frequent sites for hotspots would be useful for increasing the processing accuracy.

This paper presents a Bayesian approach for deep-network-based hotspot extraction reported in [2] to exploit the occurrence probability of hotspots caused by bone metastasis. We propose to combine the output of the deep network with a conditional probabilistic atlas of hotspots given a skeleton, because frequent sites of hotspots caused by bone metastasis depend on the type of skeleton. We show the results of applying the proposed method to 246 cases and demonstrate its effecntiveness.

\section{Methods}

The anterior and posterior bone scintigrams constitute the input. They are normalized in terms of spatial coordinates and gray values, and are forwarded to two BtrflyNets to perform skeleton and hotspot

segmentation. The conditional probability $P\left(B_{1} \mid A_{k}\right)$ of hotspot $B_{1}$ of class 1 given skeleton segmentation $A_{k}$ of the kth bone is calculated as follows:

$$
\begin{array}{ll}
\left.\mathrm{P}\left(\mathrm{B}_{1} \mid \mathrm{A}_{\mathrm{k}}\right) \quad \sum_{0}^{N} \sum_{\mathrm{n}=0}^{\mathrm{N}=0}\left(\mathrm{~A}_{\mathrm{nk}}\right) \cap \mathrm{B}_{\mathrm{nl}}\right) / & \left(\sum_{\mathrm{n}=0}^{\mathrm{N}}\left(\mathrm{A}_{\mathrm{nk}}\right) \geq 30\right) \\
0 & \text { (otherwise) }
\end{array}
$$




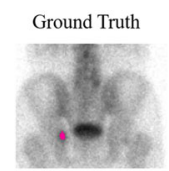

DS

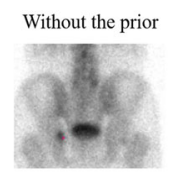

0.065

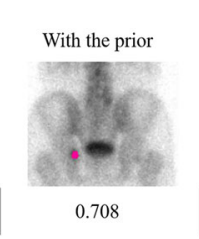

Proposed conditional probabilistic atlas

Malignant hot-spot

Fig. 1 Examples of hotspot extraction with or without the proposed conditional probabilistic atlas

the malignant hotspot. Note that the hotspot was enhanced by the proposed atlas, resulting in a reduction of false negatives and higher DS.

\section{Conclusion}

We propose a Bayesian approach for deep-network-based hotspot extraction. A conditional probabilistic atlas of hotspots caused by bone metastasis given skeleton segmentation was introduced prior to the occurrence of hotspots. The effectiveness of the proposed method was demonstrated using 246 cases in terms of the DS of hotspot segmentation in the anterior pelvic area.

\section{Acknowledgements}

This work was partly supported by MEXT/JSPS KAKENHI Grant Number JP18H03255.

\section{References}

[1] Koizumi M, Miyaji N, Murata T, Motegi K, Miwa K, Koyama M, Terauchi T, Wagatsuma K, Kawakami K, Richter J (2015) Evaluation of a Revised Version of Computer-Assisted Diagnosis System, BONENAVI Version 2.1.7, for Bone Scintigraphy in Cancer Patients, Ann Nucl Med 29:659-66

[2] Hara M, Saito A, Daisaki H, Higashiyama S, Kawabe J, Shimizu A (2020) Simultaneous process of skeleton segmentation and hot-spot extraction in a bone scintigram, International Journal of Computer Assisted Radiology and Surgery, 15 (1):23-24

\section{Self-supervised 3D-ResNet-GAN for electronic cleansing in dual-energy CT colonography}

\author{
R. Tachibana ${ }^{1,2}$, J. Näppi $^{2}$, T. Hironaka ${ }^{2}$, H. Yoshida ${ }^{2}$ \\ ${ }^{1}$ National Institute of Technology, Oshima College, Suo-Oshima, \\ Japan ${ }^{2}$ Massachusetts General Hospital/Harvard Medical School, \\ Imaging, Boston, United States
}

Keywords dual-energy CT colonography, electronic cleansing, generative adversarial network, residual block

\section{Purpose}

Early detection and removal of polyps can prevent the development of colon cancer. CT colonography (CTC) provides a safe and accurate method for examining the complete region of the colon, and it is recommended by the American Cancer Society and the United States Preventive Services Task Force as an option for colon cancer screening. Electronic cleansing (EC) performs virtual subtraction of residual materials from CTC images to enable radiologists and computer-aided detection (CADe) systems to detect polyps that could be submerged in the residual materials.

Previously, we developed a self-supervised 3D-ResNet-GAN EC scheme that used residual blocks (ResBlocks) to enhance the performance of EC based on partially self-generated training samples in single-energy CTC (SE-CTC) [1]. The 3D-ResNet-GAN EC was trained to transform an uncleansed CTC volume with tagged fecal materials into a corresponding virtually cleansed image volume. It was shown that the 3D-ResNet-GAN EC could be used with SE-CTC images to generate higher quality EC images than those obtained by our previous 3D-GAN EC scheme.

In this study, we extended the 3D-ResNet-GAN EC scheme to dual-energy CTC (DE-CTC). We also evaluated the cleansing performance of the scheme on an anthropomorphic phantom and on clinical CTC cases in comparison with those of the previous 3D-GAN EC scheme in DE-CTC [2].

\section{Methods}

An anthropomorphic phantom (Phantom Laboratory, Salem, NY) that had been designed to imitate a human colon in CT scans was filled with $300 \mathrm{cc}$ of simulated tagged fecal material, which was a mixture of aqueous fiber ( $30 \mathrm{~g}$ of psyllium), ground foodstuff ( $10 \mathrm{~g}$ of cereal), and non-ionic iodinated contrast agent (300 cc of Omnipaque iohexol, GE Healthcare). The native (empty) and partially filled versions of the phantom were scanned by use of a CT scanner (SOMATOM Definition Flash, Siemens Healthcare) with 0.6-mm slice thickness and $0.6-\mathrm{mm}$ reconstruction interval at $140 \mathrm{kVp}$ and $80 \mathrm{kVp}$ energies for acquiring the DE-CTC volumes. To simulate different concentrations of fecal tagging, the phantom was partially filled by simulated fecal materials with three contrast concentrations $(20,40$, and $60 \mathrm{mg} / \mathrm{ml})$.

Figure 1 shows the architecture of the generator network of our 3D-ResNet-GAN EC scheme. The 3D-ResNet-GAN model consists of a generator network with ResBlocks and a discriminator network. Given an uncleansed DE-CTC image pair, the generator network learns to generate the corresponding EC image volume. The architecture of the generator network is based on a 3D-U-Net architecture that has several matching down-/up-sampling convolution layers. The generator has a ResBlock on each convolutional layer in the downsampling path and between the downsampling and upsampling paths.

We trained the 3D-ResNet-GAN EC scheme iteratively with a self-supervised method that performs an initial training with a supervised-training dataset followed by an adaptive iterative selftraining with a self-training dataset that is constructed from each new input dataset [1]. Each dataset contained paired uncleansed and cleansed $128^{3}$ voxels volumes of interest (VOIs) extracted from the CT images. For the supervised-training dataset, we used 200 paired VOIs from precisely matching lumen locations of the CTC scans of the colon phantom acquired without and with $20 \mathrm{mg} / \mathrm{ml}$ and $60 \mathrm{mg} /$ $\mathrm{ml}$ contrast concentrations. For the self-training dataset, we used 100 paired VOIs from a new unseen input CTC volume, where the output VOIs matching the input VOIs were obtained by the application of the current 3D-ResNet-GAN EC.

Thus, the EC method works as follows. In the initial training, the 3D-ResNet-GAN is pre-trained with a supervised-training dataset. After this initial pre-training, we test the pre-trained 3D-ResNet-GAN model on VOIs extracted from the new unseen input case. This will generate a self-training dataset with paired VOIs from the new input

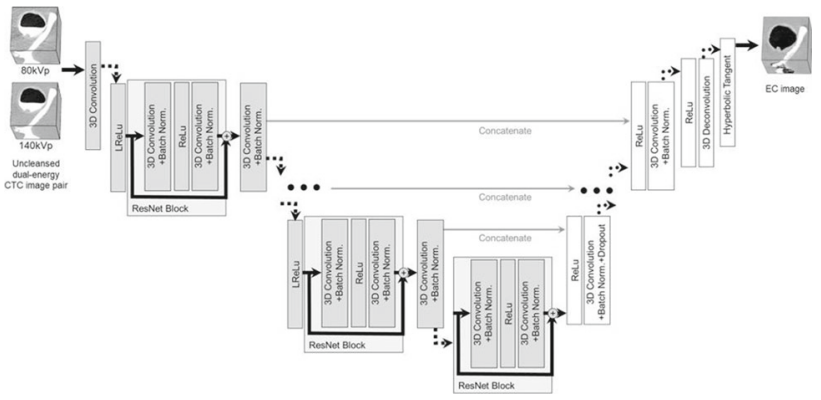

Fig. 1 Schematic architecture of the generator network of the 3DResNet-GAN EC with ResBlocks 
case. After the second training, the self-training dataset is updated by replacing the target EC VOIs with the 100 new output VOIs from the 3D-ResNet-GAN EC for the next step of the training.

For quantitative evaluation, we used the peak signal-to-noise ratio (PSNR) to assess the quality between EC VOIs and the corresponding VOIs of the native phantom. The 100 paired VOIs that were acquired with $40 \mathrm{mg} / \mathrm{ml}$ contrast agent concentration were used as the test data. To evaluate the effect of the ResBlocks and different numbers of convolution layers, we compared the performance of the proposed 3D-ResNet-GAN EC scheme with that of our previous non-ResNet 3D-GAN-EC scheme based on DE-CTC datasets. The statistical significance of the differences of PSNRs between the EC schemes was compared by use of the paired $t$ test with Bonferroni correction. Results

The image quality assessment was performed for the initial training step and for the first three self-supervised training steps (Figure 1a). The quality of the EC images progressively improved as the number of training steps increased. The proposed self-supervised 3D-ResNetGAN EC scheme yielded a higher PSNR value than that of the selfsupervised non-ResNet 3D-GAN EC scheme, except for a version of the EC scheme that was implemented with six down/up-sampling layers. The performance differences were statistically significant between the 3D-ResNet-GAN and the 3D-GAN EC schemes $\left(p<10^{-6}\right)$, except for those with six down/up-sampling layers where the performance differences were not significant in any training step.

Visual assessment of the results of clinical cases indicated that the proposed self-supervised 3D-ResNet-GAN EC scheme with six down-/up-sampling layers improves the image quality in comparison with those obtained by using our previous self-supervised 3D-GAN EC scheme (Figure 2b).

\section{Conclusion}

We developed a self-supervised 3D-ResNet-GAN scheme that uses ResBlocks to enhance the performance of EC based on partially selfgenerated training samples and DE-CTC features. Our preliminary results indicate that the scheme can generate EC images of higher quality than those obtained by the 3D-GAN EC scheme without ResBlocks. Thus, the proposed self-supervised 3D-ResNet-GAN scheme is expected to provide a high quality of EC in DE-CTC. Acknowledgements

This was study was supported partly by the NIH/NIBIB/NCI grants of R21EB024025, R01EB023942, and R01CA212382.

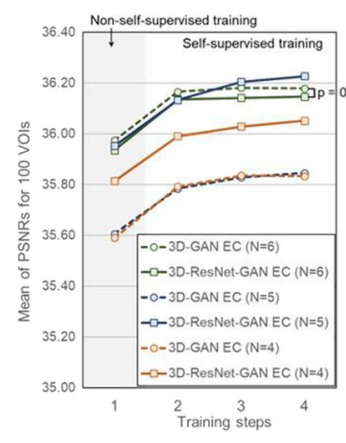

(a)

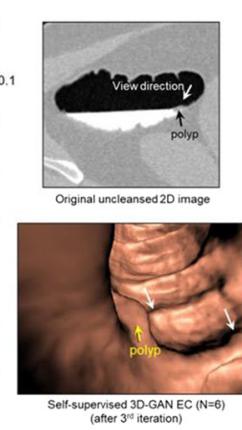

(b)

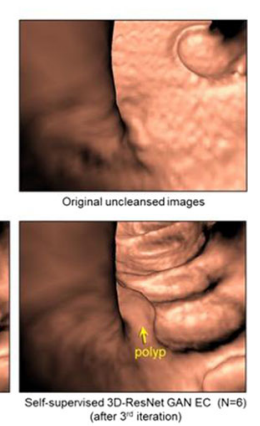

Fig. 2 a Improvement of the image quality of EC by use of the proposed self-supervised 3D-ResNet-GAN EC scheme in comparison with the previous self-supervised 3D-GAN EC scheme, where $\mathrm{N}$ indicates the number of down-/up- sampling layers. b Examples of the application of EC to a clinical CTC case with a polyp submerged in fluid. The white arrows on the 3D-GAN EC image indicate EC artifacts

\section{References}

[1] Tachibana R, Näppi JJ, Hironaka T, Yoshida H (2020) 3DResNet-GAN for improved electronic cleansing in CT colonography. International Journal of Computer Assisted Radiology and Surgery 15(Suppl 1):S213-S214

[2] Tachibana R, Näppi JJ, Hironaka T, Yoshida H (2020) Selfsupervised generative adversarial network for electronic cleansing in dual-energy CT colonography. Proc. SPIE Medical Imaging 11318:113181E.

\section{$\overline{\text { Artificial intelligence coronary calcium scoring in low }}$ dose chest CT-Ready to go?}

A. Luger ${ }^{1}$, C. Schwabl ${ }^{1}$, L. Gruber ${ }^{1}$, G. Feuchtner ${ }^{1}$, T. Sonnweber ${ }^{2}$, S. Sahanic $^{2}$, A. Pizzini ${ }^{2}$, A. Boehm ${ }^{2}$, J. Löffler-Ragg ${ }^{2}$, I. Tancevski ${ }^{2}$, M. Joannidis $^{2}$, R. Kirchmair ${ }^{2}$, E. Wöll ${ }^{3}$, G. Weiss ${ }^{2}$, E. Gizewski ${ }^{1}$, G. Widmann ${ }^{1}$

${ }^{1}$ Innsbruck Medical University, Radiology Department, Innsbruck, Austria ${ }^{2}$ Innsbruck Medical University, Internal Medicine, Innsbruck, Austria ${ }^{3}$ Zams Hospital, Internal Medicine, Zams, Austria

Keywords Coronary artery calcium score, Artificial intelligence, computer-aided diagnosis, low dose chest CT

\section{Purpose}

Low dose chest computed tomography (CT) is routinely performed for various indications such as lung cancer screening, pneumonia quantification and diagnosis of interstitial lung disease. It has been shown that coronary artery calcium score (CACS) can be derived from low dose, non-gated chest CTs as additional information about patient cardiovascular risk. Software tools detect calcium deposits, defined by a density above a threshold of $130 \mathrm{HU}$, while the radiologist manually assigns these calcium deposits to the coronary arteries, sparing calcifications of other sources like bones, aortic calcified plaques or valve calcifications. We tested an artificial intelligence (AI) prototype software for detection and quantification of coronary artery calcium volume and compared it to standard manual calcium scoring.

\section{Methods}

50 consecutive CT scans derived from the CovILD study (development of interstitial lung disease (ILD) in patients with severe SARSCoV-2 infection) were used to perform CACS assisted by the software package Syngo.via CT CaScoring (Siemens Healthineers, Erlangen, Germany) as well as by a prototype artificial intelligence network AI Rad Companion (Siemens Healthineers, Erlangen, Germany).

CT scans in low-dose setting $(100 \mathrm{kVp}$ tube potential) were acquired without ECG gating on a 128 slice multidetector CT hardware with a $128 \times 0.6 \mathrm{~mm}$ collimation, spiral pitch factor of 1.1 and image reconstruction $3 \mathrm{~mm}$ slice width. Calcium scoring was performed by two experienced radiologist and at the same time the data set was send to the cloud based AI software. Correction of threshold to $147 \mathrm{HU}$ was applied due to the low dose CT with $100 \mathrm{kVp}$ tube potential. Calcium volume values were divided into four categories $(0-9,10-99,100-499,>500)$. Correlation and comparison of group means were assessed with Kendall"s tau and Wilcoxon sign rank test. Reliability between AI and standard CACS categories was determined by weighted kappa. A two-tailed $p<0.05$ was considered statistically significant.

\section{Results}

Standard calcium volume was significantly related to AI calcium volume values, $\tau=0.909$, 95\% BA CI $(0.828-0.965), p<0.001$. Mean averages values of calcium volume was significantly higher 
detected by $\mathrm{AI}(\mathrm{M}=144.9, \mathrm{SD}=334.5)$ than by standard scoring $(\mathrm{M}=129.2, \mathrm{SD}=316.3), \mathrm{T}=389.5, p<0.001$.

Weighted kappa showed a very good reliability for the AI categories $(\kappa=0.90)$, however, AI lead to a shift of five patients into higher strata compared to standard CACS (10\%).

Of all patients with no detectable calcium volume AI classified all but one correctly as zero (95.2\%). In this patient small valve calcification adjacent to the coronary artery was misclassified. AI did not detect calcium deposits in four patients $(8 \%)$, which had a very low calcium volume $(\mathrm{M}=1.2, \mathrm{SD}=1.1)$.

Conclusion

AI CACS can be routinely obtained from low dose non-gated chest CTs and add value for patient risk stratification.

\section{A computer-aided diagnosis system based on 3-D attention-CNN architecture for tumor diagnosis on automated breast ultrasound}

\author{
R. F. Chang ${ }^{1,2}$, J. Zhang ${ }^{2}$, Y. S. Huang ${ }^{1}$ \\ ${ }^{1}$ National Taiwan University, Department of Computer Science \\ and Information Engineering, Taipei, Taiwan ${ }^{2}$ National Taiwan \\ University, Graduate Institute of Biomedical Electronics \\ and Bioinformatics, Taipei, Taiwan
}

Keywords Automated Breast Ultrasound, Computer-aided Diagnosis, Convolution Neural Network, Attention Mechanism

\section{Purpose}

Automated breast ultrasound (ABUS) is a widely used screening modality in detecting and diagnosing breast cancer. It could scan the whole breast and provide the complete breast three-dimensional (3-D) volume. It is a time-consuming task for a radiologist to diagnose tumors by reviewing an ABUS image. Moreover, misjudgment exists if the radiologist interprets cancer only with the information offered by ABUS. Therefore, the computer-aided diagnosis (CADx) systems developed with texture or morphology features are proposed to assist the radiologist. Recently, convolutional neural networks (CNN), which extract features automatically, have been widely used in medical images, and the CNN-based CADx could achieve outstanding performance. Hence, in the study, a CADx system developed with3-D CNN architecture is proposed for ABUS tumor classification. Methods

The proposed CADx system for tumor diagnosis consists of the VOI extraction, a 3-D tumor segmentation model, and a 3-D tumor classification model. The volumes of interest (VOIs) are defined by an experienced radiologist and resized to fixed-size first in the VOI extraction. Second, in the tumor segmentation, a 3-D U-Net ++ is applied to the resized VOI to create the tumor mask. Finally, the VOI, the enhanced VOI using the histogram equalization, and the corresponding tumor mask are fed into the tumor classification model. Our tumor classification model is constructed with different Inception blocks. However, there are many redundant features also regarded as useful features in the subsequent classification process. Hence, the attention mechanism, the squeeze-and-excitation (SE) model, is embedded in our classification model to select critical elements for classifying the tumor as benign or malignant.

\section{Results}

This study collected the used dataset from collected by InveniaTM automated breast ultrasound system (Invenia ABUS, GE Healthcare). An automatic linear broadband transducer obtains all ABUS volumes with a covering area of $15.4^{\prime} 17^{\prime} 5 \mathrm{~cm}$. Each ABUS volume is made of 330 serial 2-D images, and each 2-D image consists of $831 \times 422$ pixels, and the distance between each slice is $0.5 \mathrm{~mm} .396$ patients (age $49.2 \pm 10.3$ years) with 444 pathology-proven tumors, including 226 malignant and 218 benign lesions, are utilized in our experiments. To estimate the system performance, three indices, including accuracy, sensitivity, and specificity, are used to validate our CADx system. Moreover, to ensure the robustness and reliability of CADx, fivefold cross validation is performed in our system training and testing. In our experiments, the proposed system could achieve $85.6 \%$ of accuracy, $85.0 \%$ of sensitivity, and $86.2 \%$ of specificity. In conclusion, the results imply that our system can classify tumors as malignant or benign.

\section{Conclusion}

A CADx system composed of the VOI extraction, a 3-D tumor segmentation, and a 3-D SE-Inception classification model is proposed for tumor classification on ABUS volumes. First, in VOI extraction, the VOIs are cropped by an experienced radiologist and resized into fixed-size. Second, the tumor masks are outlined by a 3-D U-net ++ model. Finally, VOIs and masks are fed into the 3-D SEInception model to extract useful features for diagnosing tumors. The proposed CADx system takes advantage of texture and morphology for medical images, and the overall performance shows the proposed CADx has a capability for tumor classification on ABUS volumes.

\section{Developing a computer-aided diagnosis system by 3-D SE-ResNeXt for lung nodule classification on low-dose computed tomography}

\section{Y. S. Huang ${ }^{1}$, S. Z. Huang ${ }^{2}$, T. C. Lin ${ }^{1}$, R. F. Chang ${ }^{3,4}$}

${ }^{1}$ National Taiwan University, Computer Science \& Information Engineering, Taipei, Taiwan ${ }^{2}$ National Taiwan University, Graduate Institute of Networking and Multimedia, Taipei, Taiwan ${ }^{3}$ National

Taiwan University, Department of Computer Science and Information Engineering, Taipei, Taiwan ${ }^{4}$ National Taiwan University, Graduate Institute of Biomedical Electronics and Bioinformatics, Taipei, Taiwan

Keywords Low-dose Computed Tomography, Computer-aided Diagnosis, Convolution Neural Network, Squeeze-and-Excitation

\section{Purpose}

Lung cancer was with the highest mortality rate in the world. Lowdose Computed Tomography (LDCT) is an essential tool for lung cancer detection and diagnosis. It can provide a complete three-dimensional (3-D) chest image. Although the LDCT was a useful lung examination modality, the different nodule decision rules and radiologist's experience would result in different diagnosis results. Therefore, the computer-aided diagnosis (CADx) system was developed for assisting radiologist. In recent, designing a CADx system based on the convolution neural network (CNN) in the medical image has become a tendency toward cancer diagnosis due to the powerful capability of automatic feature extraction and classification. Many studies have proven that a CADx system with $\mathrm{CNN}$ could help radiologists to make a preliminary diagnosis. Therefore, in this research, a CADx system that used an advanced residual network, ResNeXt block, as the backbone was proposed for lung nodule diagnosis. Moreover, to prevent the ResNeXt block from generating redundant features, a lightweight and attention mechanism module, squeeze-and excitation (SE), was embedded in the ResNeXt block to help focus on important features.

\section{Methods}

In this research, the proposed CADx system for lung nodule diagnosis consists of the VOI extraction and a 3-D attention ResNext nodule classification model, which integrates ResNeXt block with attention machine module. The volumes of interest (VOIs) are defined and normalized into the range from -1 to 1 first in the VOI extraction. In the nodule classification model, the ResNeXt block inspired by the ResNet block and the split-transform-merge strategy of InceptionNet 
is built, and the SE module is embedded into the block for developing the 3-D SE-ResNeXt classification model. The defined VOIs are then delivered to the 3-D SE-ResNeXt model to determine nodule as malignant or benign.

\section{Results}

In this research, the materials were collected from the National Lung Screening Trial (NLST). All participants have received three screenings, including GE Healthcare, Philips Healthcare, Siemens Healthcare, and Toshiba machine brands. The number slice of LDCT volume was between 100 and 300, and the range of pixel spacing was between 0.5 and 1 , and the range of slice thickness was between 1 and 3 . The used dataset consists of 880 nodules, including 440 malignant and 440 benign. In malignant, there are 171 lesions smaller than $1 \mathrm{~cm}$ and 269 lesions larger than $1 \mathrm{~cm}$, whereas there are 398 nodules smaller than $1 \mathrm{~cm}$ and 42 nodules larger than $1 \mathrm{~cm}$ in benign. For system validation, three performance indices, including accuracy, sensitivity, specificity, and fivefold cross validation, are used to validate our CADx system. In experiments, the proposed system's accuracy, sensitivity, and specificity are $84.7 \%, 83.6 \%, 85.7 \%$. In conclusion, the results indicate that the proposed system has the capability for discriminating malignant nodules from benign ones.

\section{Conclusion}

In this study, a CADx system made of the VOI extraction and the 3-D $\mathrm{CNN}$-based classification model is proposed for lung nodule classification in LDCT images. First, the VOIs are defined, and then the VOIs are fed into the 3-D Att-ResNeXt for determining nodule types. The proposed CADx system takes advantage of CNN for medical images, and the overall performance of this study may be further improved by using other CNN models.

\section{Computer-aided diagnosis of X-ray thorax diseases using deep convolutional neural network with graph attention mechanism}

\author{
Y. W. Lee ${ }^{1}$, S. K. Huang ${ }^{2}$, R. F. Chang ${ }^{1,2,3}$ \\ ${ }^{1}$ National Taiwan University, Department of Computer Science \\ and Information Engineering, Taipei City, Taiwan ${ }^{2}$ National Taiwan \\ University, Department or Graduate Institute of Networking \\ and Multimedia, Taipei City, Taiwan ${ }^{3}$ National Taiwan University, \\ Graduate Institute of Biomedical Electronics and Bioinformatics, \\ Taipei City, Taiwan
}

Keywords Chest X-ray, Thorax diseases, Computer-aided diagnosis, Graph attention mechanism

\section{Purpose}

According to global statistical results for leading causes of life loss in 2017, chronic obstructive pulmonary disease (COPD) (including emphysema) and lung cancer were the seventh and twelfth of the leading causes, respectively. Clinical researches [1] indicate that early detection and treatment could mitigate the mortality rate of COPD. Hence, early detection and treatment are important for patients with thorax disease. In the chest radiograph, the radiologic features are useful to diagnose thorax diseases, and each thorax disease has different radiologic features. Some clinical researches indicate that with the help of the computer-aided diagnosis (CAD) system, the diagnostic performance of junior radiologists could be improved. The diagnosis performance of radiologists with the CAD system for malignant lung nodules has better sensitivity and false-positive rate [2]. Besides, the CAD system might detect some missed finding by humans and reduce the reader-dependent problem between radiologists. Therefore, detecting thorax diseases with the CAD system is useful for clinical. Taking the benefits of deep learning techniques, the CAD system's diagnosis performance could be almost similar to radiologists and the related methods have been extended to employed

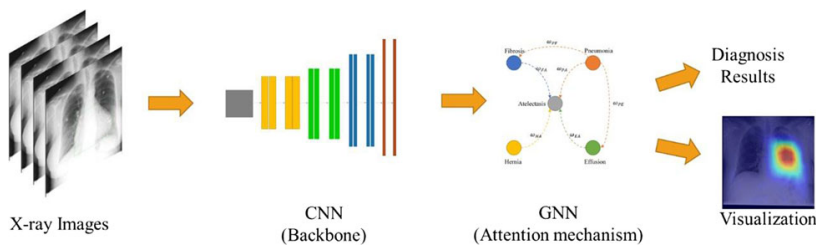

Fig. 1 The overall flow chart of our proposed X-ray thorax CAD system

in different topics. In this study, we proposed a CAD system to diagnose thorax diseases using chest X-ray images through the $\mathrm{CNN}$ model and the graph neural network (GNN) model with the graph attention mechanism.

\section{Methods}

In this study, we proposed a CAP system for diagnosing thorax diseases using chest X-ray images, which contains a feature extractor and GNN with the graph attention mechanism, called CGAM (CNN Model with graph attention mechanism). First, we employed the CNN backbone (EfficientNet) model to extract the detailed image representation information. Second, the GNN with the graph attention mechanism was used to enhance the correlation between thorax diseases. Finally, the CAD system predicts the diagnostic results and the visualization results (gradient-weighted class activation mapping, Grad-CAM). Figure 1 shows the overall flow chart of our proposed $\mathrm{X}$-ray thorax CAD system.

\section{Results}

In this study, the open dataset (NIH Chest X-ray dataset) was employed to diagnose thorax diseases in X-ray images, which contained 112,120 frontal-view chest X-ray images, labels with 14 common thorax diseases. The data ratio for the training set, validation set, and test set were 7:1:2, respectively. In this study, we used the AUC (area under the receiver operating characteristic (ROC) curve) score for evaluating model performance. In our model (CGAM-EfficientNet-B4) the AUC scores of atelectasis, cardiomegaly, effusion, infiltration, mass, nodule, pneumonia, pneumothorax, consolidation, edema, emphysema, fibrosis, pleural thickening, hernia, and average were $0.7817,0.8834,0.8337,0.7049,0.8330,0.7840,0.7371,0.8689$, $0.7525,0.8515,0.9424,0.8365,0.7957,0.9420,0.8248$, respectively. Conclusion

The chest X-ray is one of the most common imaging examinations in clinical diagnosis, and chest $\mathrm{X}$-ray images are useful to diagnose different thorax diseases. In this study, we proposed a CNN model combined with GNN and the graph attention mechanism to diagnose thorax diseases using chest X-ray images. The experiment results show convolution neural network models can effectively predict different thorax diseases. In this study, we proposed a CAD system to diagnose thorax diseases using chest X-ray images with clinical values.

\section{References}

[1] Csikesz NG, Gartman EJ (2014) New developments in the assessment of COPD: early diagnosis is key. International journal of chronic obstructive pulmonary disease, vol. 9, pp. 277-286.

[2] Hwang EJ, Nam JG, Lim WH, Park SJ, Jeong YS, Kang JH, Hong EK, Kim TM, Goo JM, Park S, Kim KH, Park CM (2019) Deep Learning for Chest Radiograph Diagnosis in the Emergency Department. Radiology, vol. 0, pp. 191225. 


\section{Poster Session}




\section{Can radiological technologists create training data for automatically segmenting the mammary gland region in non-dense breasts?}

M. Yamamuro ${ }^{1}$, Y. Asai ${ }^{1}$, N. Hashimoto ${ }^{1}$, N. Yasuda ${ }^{1}$, Y. Ozaki ${ }^{2}$, S. $\mathrm{Nin}^{3}$, K. Ishii ${ }^{3}$, T. Yamada ${ }^{4}$, M. Nemoto ${ }^{5}$, Y. Kimura ${ }^{6}$, H. Handa ${ }^{7}, \mathrm{H}$.

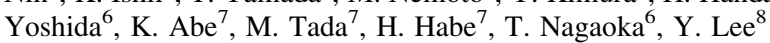

${ }^{1}$ Kindai University Hospital, Radiology Center, Osaka-sayama, Japan

${ }^{2}$ Kyoto Prefectural Police Headquarters, Research Institute

of Scientific Investigation, Kyoto, Japan ${ }^{3}$ Kindai University Faculty of Medicine, Department of Radiology, Osaka-sayama, Japan ${ }^{4}$ Kindai University, Division of Positron Emission Tomography, Osakasayama, Japan ${ }^{5}$ Kindai University, Department of Biomedical Engineering, Kinokawa, Japan ${ }^{6}$ Kindai University, Department of Computational Systems Biology, Kinokawa, Japan ${ }^{7}$ Kindai University, Department of Informatics, Higashi-osaka, Japan ${ }^{8}$ Niigata University, Graduate School of Health Sciences, Niigata, Japan

Keywords Mammography, Breast density, Mammary Gland Region, Segmentation

\section{Purpose}

Although a quantified breast density is an effective index for individualized screening mammography, the technique has not yet been realized because of the differences in the perceptions for mammary gland region among practitioners. Recently, a deep learning technique has been applied to segment a mammary gland region in two-dimensional mammograms to achieve a reliable individualized breast cancer screening based on an accurate breast density measurement, instead of relying on human vision. In contrast, a large amount of ground truth (prepared by mammary experts) is required for highly accurate deep-learning practice; however, this work is time- and labor-intensive. If multiple radiological technologists can share the process for producing training images in deep learning practice independent of their certified level, we can more easily prepare a large number of training images to construct a segmentation model. In a previous study, we investigated the differences in the acquired mammary gland regions among three radiological technologists (hereafter referred to as practitioners) having different experiences and reading levels to streamline the ground truth in deep learning, who shared the segmentation criteria based on the Breast Imaging Reporting and Data System (BI-RADS). As a result, a good agreement was found among the segmented mammary gland regions; however, only dense breasts were used in that study because of easy visual recognition of the mammary gland tissue. In this study, we tried an identical experiment from the previous study for non-dense breasts in which visually recognizing the mammary gland region was difficult.

\section{Methods}

A total of 150 mediolateral oblique-view mammograms of Japanese women, who underwent digital mammography from 2017 to 2018 with normal breasts, were used in this study. All mammograms were assessed as scattered mammary gland or fatty breasts, that is, nondense breasts, based on a BI-RADS criterion. All images were radiographed with the Canon Pe-ru-ru mammographic system, equipped with a target/filter of molybdenum/molybdenum (for 20-mm breast thickness).

We investigated the degree of agreement among mammary gland regions segmented by three radiological technologists as $\mathrm{A}, \mathrm{B}$, and $\mathrm{C}$ with 20,10, and 1 year of certified experience in mammography, respectively. This investigation was performed in the following four steps: (1) Three certified radiological technologists were stringently lectured regarding the segmentation criteria of the mammary gland region by a certified radiologist, with $>20$ years of experience, using 20 non-dense breast images. For example, the mammary gland region
Table 1 Results of six assessment factors on segmented mammary gland regions

\begin{tabular}{|c|c|c|c|c|c|c|}
\hline \multirow[t]{2}{*}{ Practitioner } & \multirow[t]{2}{*}{$\begin{array}{l}\text { Breast } \\
\text { density } \\
(\%)\end{array}$} & \multirow[t]{2}{*}{$\begin{array}{l}\text { M G D } \\
(\mathrm{mGy})\end{array}$} & \multirow[t]{2}{*}{$\begin{array}{l}\text { Area } \\
\left(\mathrm{mm}^{2}\right)\end{array}$} & \multirow[t]{2}{*}{$\begin{array}{l}\text { Mean } \\
\text { pixel } \\
\text { value }\end{array}$} & \multicolumn{2}{|c|}{$\begin{array}{l}\text { Central } \\
\text { coordinates } \\
(\mathrm{mm})\end{array}$} \\
\hline & & & & & $X$ & $\mathrm{Y}$ \\
\hline \multicolumn{7}{|c|}{ 1st time experiment } \\
\hline A & 12 & 1.24 & 7192 & 652 & 85.8 & 115.0 \\
\hline $\mathrm{B}$ & 10 & 1.19 & 7004 & 661 & 86.1 & 118.6 \\
\hline $\mathrm{C}$ & 6 & 0.95 & 8923 & 690 & 85.2 & 113.0 \\
\hline$|\mathrm{A}-\mathrm{B}|$ & 2 & 0.05 & 188 & 9 & 0.3 & $3.6^{*}$ \\
\hline $\mid \mathrm{A}-\mathrm{Cl}$ & $6^{*}$ & $0.29 *$ & $1731 *$ & $38^{*}$ & 0.6 & 2.0 \\
\hline $\mid \mathrm{B}-\mathrm{Cl}$ & $4 *$ & $0.24 *$ & $1919 *$ & $29 *$ & 0.9 & $5.6^{*}$ \\
\hline \multicolumn{7}{|c|}{ 2nd time experiment } \\
\hline A & 12 & 1.30 & 6529 & 649 & 85.2 & 115.6 \\
\hline $\mathrm{B}$ & 10 & 1.30 & 6898 & 658 & 85.9 & 116.8 \\
\hline $\mathrm{C}$ & 12 & 1.32 & 7375 & 652 & 85.0 & 113.9 \\
\hline$|\mathrm{A}-\mathrm{B}|$ & 2 & 0.00 & 369 & 9 & 0.7 & 1.2 \\
\hline $\mid \mathrm{A}-\mathrm{Cl}$ & 0 & 0.02 & $846^{*}$ & 3 & 0.2 & 1.7 \\
\hline $\mid \mathrm{B}-\mathrm{Cl}$ & 2 & 0.02 & 477 & 6 & 0.9 & $2.9^{*}$ \\
\hline
\end{tabular}

$* p<0.05$

converges on the nipple conically, and the existence and characteristics of the retromammary space. The lecture was followed by a discussion on the criteria among the three practitioners. (2) They attempted to independently segment the mammary gland region in 150 non-dense breast images, including the aforementioned 20 lecture images (this was called the 1st time experiment). (3) Six months after the 1 st time experiment, a lecture by the same radiologist was held again using the same images, and an identical experiment with the 1st time experiment was performed again (this was called the 2nd time experiment). (4) The degree of agreement in the segmented mammary gland region among the three practitioners in the 1st and 2nd time experiments was assessed according to the following six factors: breast density, mean glandular dose, region size, mean pixel value, and central coordinate (X- and Y-directions). The breast density and mean glandular dose were calculated using our original method [1] and the Dance formula [2], respectively. The other assessment factors were obtained using ImageJ software. The presence of significant differences was judged via Bland-Altman analysis (for breast density) and Student's t-test with Bonferroni correction (for all assessment factors).

\section{Results}

In the 1st time experiment, the breast densities obtained from the mammary gland regions segmented by practitioners " $A$ " and " $\mathrm{B}$ " were in good agreement, and all assessment factors, except for the central coordinate for the $\mathrm{Y}$ direction, showed no significant differences. Bland-Altman plot also revealed no fixed or proportional bias in the breast densities derived from "A" and "B." In contrast, the breast density derived from the most inexperienced practitioner " $\mathrm{C}$ " was significantly lower than that from the other two practitioners because of the large region.

In the 2nd time experiment, the degree of agreement between the segmented regions by "A" and "B" was even higher, and all assessment factors showed no significant differences. The results of the $t$-test are listed in Table 1. Figure 1 shows the Bland-Altman plot for breast density between "A" and "B." There was no fixed bias or 


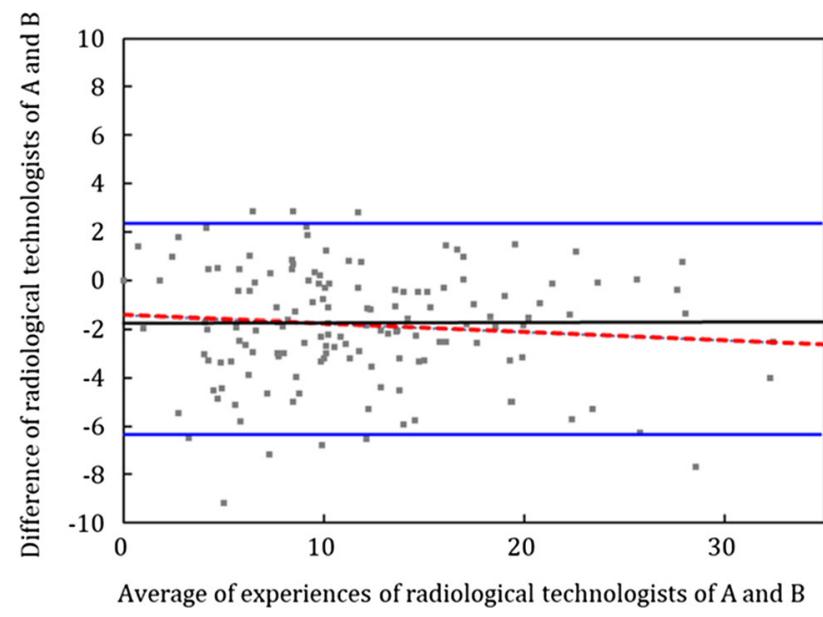

Fig. 1 Bland-Altman plot of practitioner A versus B for breast density in the 2 nd time experiment. The mean of difference between $\mathrm{A}$ and $\mathrm{B}$ is -1.82 (black solid line), the upper and lower limits of the $95 \%$ confidence interval are 2.77 and -6.41 , respectively (blue solid lines), and the slope of the regression line is -0.0344 (red broken line)

proportional bias, and the 95\% confidence interval narrowed from 11.14 in the 1 st time experiment to 9.18 . The breast densities derived from practitioner " $\mathrm{A}$ " and "B" can be deemed as identical. In addition, the result of practitioner " $\mathrm{C}$ " was also significantly improved by performing two lectures with a long interval. After receiving the second lecture, the significant difference between " $\mathrm{B}$ " and " $C$ " disappeared. Thus, the two-step lecture is an avenue for criteria sharing for segmenting the mammary gland region, especially for inexperienced practitioners.

\section{Conclusion}

We concluded that certified radiological technologists can create the training data for deep learning that satisfies a certain criteria by receiving one- or two-time lecture regarding mammary gland region segmentation from a certified radiologist. This leads to an increase in training images for highly accurate deep-learning practice.

\section{Acknowledgements}

This work was supported by JSPS Grants-in-Aid Scientific Research, Grant Number JP18K07736.

\section{References}

[1] Yamamuro M, Asai Y, Yamada K, Ozaki Y, Matsumoto M, Murakami T (2019) Prediction of glandularity and breast radiation dose from mammography results in Japanese women. Medical \& Biological Engineering \& Computing 57(1):289-98

[2] Dance DR., Skinner CL., Young KC., Beckett JR., Kotre CJ (2000) Additional factors for the estimation of mean glandular breast dose using the UK mammography dosimetry protocol. Physics in Medicine and Biology 45:3225-40 $\overline{\text { Deep learning for automatic quantification of } \mathrm{AVN}}$ of the femoral head on 3D MRI in patients eligible for joint preserving surgery: a pilot study

A. Ruckli ${ }^{1}$, F. Schmaranzer ${ }^{2}$, T. Lerch $^{2}$, A. Boschung ${ }^{2}$, S. Steppacher ${ }^{3}$, J. Burger ${ }^{1}$, M. Tannast ${ }^{4}$, K. Siebenrock ${ }^{3}$, N. Gerber ${ }^{1}$, K. Gerber ${ }^{1}$

${ }^{1}$ University of Bern, sitem Center for Translational Medicine and Biomedical Entrepreneurship, Personalised Medicine research group, Bern, Switzerland ${ }^{2}$ Inselspital, University of Bern, Department of Diagnostic-, Interventional- and Pediatric Radiology, Bern, Switzerland ${ }^{3}$ Inselspital, University of Bern, Department of Orthopaedic Surgery and Traumatology, Bern, Switzerland ${ }^{4}$ Kantonsspital Fribourg, University of Fribourg, Department of Orthopaedic Surgery and Traumatology, Fribourg, Switzerland

Keywords necrosis, hip, segmentation, deep learning

\section{Purpose}

Size of necrosis is an important prognostic factor in the management of femoral head necrosis (AVN), usually estimated on radiographs and MRI which is subjective and requires experienced physicians. Ideally, a fast-volumetric assessment of necrosis size would be desirable for a more objective standardized evaluation. Thus, we evaluated a deep-learning method to automatically quantify the necrotic bone in AVN.

\section{Methods}

IRB-approved retrospective study of 34 patients (mean age 30 years, 14 women) with AVN according to the commonly recommended 2019 ARCO grading: I (negative X-rays): 3 hips; II (no fracture): 5 hips; IIIA (head collapse $2 \mathrm{~mm}$ ): 12 hips. Patients underwent preoperative 3T hip MRI including $0.8 \mathrm{~mm}^{3}$ 3D T1VIBE on which manual ground truth segmentation of necrosis and the vital bone from the femoral head was performed by an expert reader and then used to train a set of convolutional neural networks (nnU-Net [1]). The raw data had a median image shape and spacing of $104 \times 384 \times 384$ voxels and $1 \times 0.44 \times 0.44 \mathrm{~mm}$, respectively. The highest in-plane resolution was oriented axial-oblique parallel to the femoral neck. As a preprocessing step, the images were resampled to the medial spacing and volume cropped around the femoral head center to the shape of $80 \times 160 \times 160$ voxels. Volume cropping reduced the background complexity and accelerated the network training time. A fivefold cross-validation was performed between manual and automatic volumetric analysis of absolute/relative necrosis volume. The mean difference between manual and automatic segmentation was compared with paired t-tests and correlation was assessed with Pearson correlation coefficients. We compared the absolute and relative size of the necrosis between early and advanced stages of AVN (ARCO I/II versus IIIA/B) using Mann-Whitney U tests. A p-value $<0.05$ determined statistical significance.

\section{Results}

The best performing configuration was the ensemble of the $2 \mathrm{D}$ and 3D U-net. The mean Dice coefficient for the vital femoral head bone and necrosis was $89 \pm 9 \%$ and $69 \pm 25 \%$, respectively. The individual $2 \mathrm{D}(89 \pm 9 \%, 67 \pm 23 \%)$ and $3 \mathrm{D}(89 \pm 10 \%, 69 \pm 26 \%)$ networks were performing very similarly on both vital and necrotic bone $(p>0.05)$. Mean absolute and relative AVN volume was comparable between manual $\left(8.2 \pm 7.4 \mathrm{~cm}^{3}, 17 \pm 15 \%\right)$ and automatic $\left(7.3 \pm 6.7 \mathrm{~cm}^{3}, 15 \pm 14 \%\right)$ segmentation (both $\left.p>0.05\right)$ and showed a strong correlation $(\mathrm{r} p=0.90$ and $\mathrm{r} p=0.92$, both $p<0.001$ ), respectively. Manual and automated segmentation detected a difference (both $p<0.05$ ) in relative necrosis volume between early and advanced AVN: $8 \pm 8 \%$ vs. $20 \pm 16 \%$ and $7 \pm 8 \%$ vs. $18 \pm 14 \%$, respectively. 


\section{Conclusion}

Due to the large number of parameters, it is known that 3D convolution networks have a less good generalization performance compared to the 2D counterparts. Even if the 3D context is essential this often makes both networks perform very similar. So far, it seems that the deep learning approach cannot benefit from the 3D MRI sequence. Future additional tests and 2D MRI sequences are required to reject or confirm this hypothesis. However, applying a deep learning method for volumetric assessment of AVN is feasible and showed very strong agreement and enabled to distinguish early versus advanced disease stages which paves way for evaluation in larger datasets, with the goal to determine its prognostic value.

\section{References}

[1] Isensee F, Jaeger PF, Kohl SAA, Petersen J, Maier-Hein K H (2020) nnU-Net: A Self-Configuring Method for Deep LearningBased Biomedical Image Segmentation. Nature Methods: 1-9

\section{AI-assisted RoI extraction software tool for radio- therapy simulation}

\author{
A. Kimura ${ }^{1}$, M. T. Nadege ${ }^{2}$ \\ ${ }^{1}$ Ashikaga University, Faculty of Engineering, Ashikaga, Tochigi, \\ Japan ${ }^{2}$ Ashikaga University, Graduate School of Engineering, \\ Ashikaga, Tochigi, Japan
}

Keywords RoI, AI, radiotherapy simulation, Geant4

\section{Purpose}

Monte Carlo radiation simulations have been used in areas of handling radiations accurately, such as high energy physics, nuclear physics, accelerator physics, medical science, and space science. It takes charge of the important role in high energy physics in order to verify a theory, explore an unknown particle, and so on. In radiotherapy, it has been used it in order to precisely evaluate dose distributions in an irradiated human body constructed from medical data or optimize devices of a beam delivery system [1].

Geant 4 toolkit has been used for a Monte Carlo simulation of the passage of particles through matter [2]. It has been done physics validations and developed as a $\mathrm{C}++$ class library for developing user software applications. Therefore, it comes to be used for medical physics simulations.

We had developed and released gMocren since 2006. It has reached over 2400 downloads since the release. It is volume visualization software for Geant4-based radiotherapy simulations. It had been designed according to the requirements of medical users for the visualization system of Geant 4 . gMocren can visualize fused images of patient data, a dose distribution, particle trajectories and treatment apparatus with $3 \mathrm{D}$ view and 3 cross-sectional sliced images. It can only visualize RoIs, and it has no capability to edit RoIs and create a DVH.

In this research, an RoI editing software tool using NVIDIA Clara has been developing. It supports extracting region of tumors using an artificial intelligence from medical image data such as DICOM dataset and editing RoIs for radiotherapy simulation. It is useful to create RoIs and a DVH for Geant4-based radiotherapy simulation.

Methods

NVIDIA Clara is a healthcare application framework for AI-powered imaging and genomics. It uses NIVIDA GPU to accelerate extracting tumor or organ regions from medical images. NVIDIA provides pretrained models due to tumor or organ extractions.

In user requirements of radiotherapy simulations, functions of importing RoI data and creating DVH are required in order to analyze outcomes of a radiotherapy simulation. Geant4 toolkit has not
Table 1 A Linux server specification for NVIDIA Clara

\begin{tabular}{ll}
\hline CPU & AMD Ryzen 7 2700 \\
GPU & NVIDIA GeForce RTX 2080 \\
Main memory & 16 GB \\
Linux OS & Ubuntu 18.04.3 LTS \\
CUDA Toolkit & 10.2 .89 \\
Docker & NIVIDA Docker 2.0.3 \\
\hline
\end{tabular}

provided those functions as an analysis tool. Therefore, medical users create RoI and DVH by using such a treatment planning system in a hospital. The RoI editing tool will be freely available, so it is useful and available not only for students or beginners but also researchers using Geant4-based radiotherapy simulation.

The RoI editing tool has function to export RoI data to DICOMRT Structure or DICOM-RT Dose files. gMocren can visualizes extracted organ regions with DICOM-RT Structure Set file, such as outlines of a human body, organs, tumors, and so on. The RoIs are drawn on a medical image used in the tissue extraction. The RoI editing tool is implemented by using Python and HTML5.

NVIDIA Clara is used to extract initial RoIs in the RoI editing tool. It is possible to edit RoI shapes from the initial RoIs. The tumor region of edited RoI will be able to return to and update the trained $\mathrm{AI}$ model of NVIDIA Clara.

\section{Results}

NVIDIA Clara is provided as a docker container for a Linux PC with a NVIDIA GPU [5]. It can work on a Linux PC as a standalone AI server and provides an HTML interface to communicate with the other software. A Linux PC shown in Table 1 is used for NVIDIA Clara server.

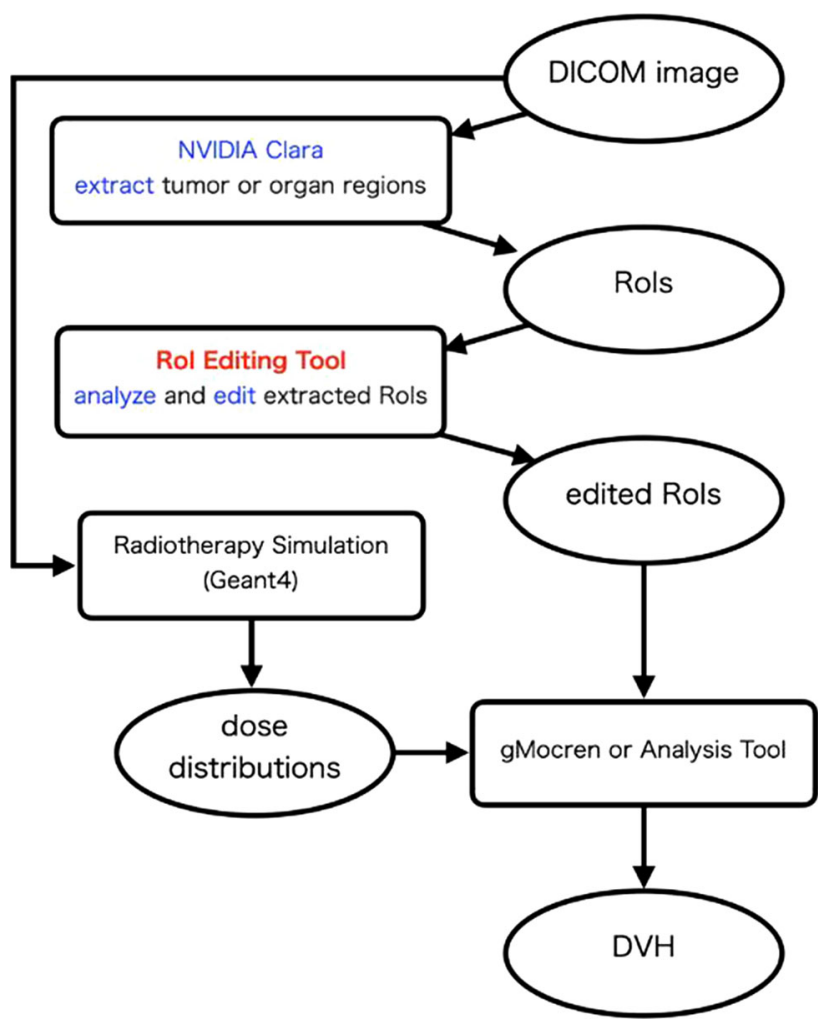

Fig. 1 Process of creating DVH from DICOM image dataset with Geant4-based radiotherapy simulation 
A spleen region in DICOM images can be extracted adequately by using NVIDIA Clara and MITK (Medical Image Interaction Toolkit). A spleen region is extracted from chest region of a patient dataset within $10 \mathrm{~s}$. The size of the patient dataset is $512 \times 512 \times 34$ voxels.

The process to create DVH from DICOM image dataset with radiotherapy simulation is shown in Fig. 1. NVIDIA Clara extracts tumor or organ regions in DICOM Image. The extracted regions are used as initial RoIs data. User analyzes and edits the extracted RoIs with the RoI editing tool. And Geant4-based radiotherapy simulation calculates dose distributions with the DICOM image dataset. Finally, a DVH is calculated with the edited RoIs and the calculated dose distributions and visualized by using gMocren or an analysis tool.

\section{Conclusion}

The RoI Editing tool using AI has been developing. NVIDIA Clara is used as an AI server to extract tumor or organ regions in medical image dataset such as a DICOM dataset. The extracted region is used to create RoIs, and then the RoIs can be edited by user. The RoI editing tool will be available as standalone software. Therefore, it is useful and available not only for students or beginners but also researchers using Geant4-based radiotherapy simulation.

\section{References}

[1] Archambault L, Beaulieu L, Carrier JF, Castrovillari F, Chauvie S, Foppiano F, Ghiso G, Guatelli S, Incerti S, Lamanna E, Larsson S, Lopes MC, Peralta L, Pia MG, Rodrigues P, Tremblay VH, Trindade A (2003) Overview of Geant4 Applications in Medical Physics. Nuclear Science Symposium Conference Record, 2003 IEEE, Vol.3, 19-25, pp. 1743-1745

[2] GEANT4 Collaboration (S. Agostinelli et al.) (2003) 'GEANT4: A Simulation Toolkit," Nucl. Instrum. Meth. A 506: pp. 250-303.

\section{Remote interventional support for emergency care application mobile ultrasound}

M. Kranzfelder ${ }^{1}$, M. Gharba ${ }^{2}$, T. Vogel ${ }^{1}$, H. Feussner ${ }^{1}$, D. Wilhelm ${ }^{1}$, H. Friess ${ }^{3}$, J. Eichinger ${ }^{2}$, D. Ostler ${ }^{4}$

${ }^{1}$ Klinikum rechts der Isar, TU München, Department of Surgery, München, Germany ${ }^{2}$ Huawei Technologies Duesseldorf GmbH, Munich Office, European Research Center, München, Germany ${ }^{3}$ Klinikum rechts der Isar, TU Munich, Department of Surgery, München, Germany ${ }^{4}$ Klinikum rechts der Isar, TU Munich, Researchgroup MITI, München, Germany

Keywords 5G, Telemedicine, Ultrasound, Emergency care

\section{Purpose}

Preclinical patient treatment in an acute emergency situation (e.g. car accident) benefits greatly from support by a medical expert. Especially in rural areas, with a lack of trained medical staff and long travel distances to the hospital, a fast and reliable preclinical diagnosis, e.g. by mobile ultrasound, significantly increases patient safety. This support with virtual presence of a medical expert can be achieved by tele-medical applications, enabling bi-directional data communication between the first responder and the hospital.

\section{Methods}

Tele-medical applications require reliable, fast and secure wireless data transmission. 5th. generation mobile communication (5G) facilitates an up to $100 \times$ higher data rate (up to $10.000 \mathrm{MBit} / \mathrm{s}$ ), up to 200x higher data capacity and a (very) low latency (Ping $<1 \mathrm{~ms}$ ) compared to $4 \mathrm{G} / \mathrm{LTE}$. Previous research has shown, that $5 \mathrm{G}$ data

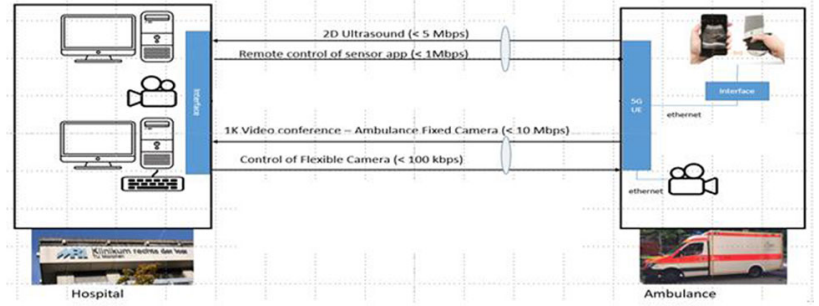

Fig. 1 Bi-directional ultrasound, video and audio data transfer between stationary unit (hospital) and moving point of care (ambulance)

transmission volume, rate, and latency met the requirements for realtime track and trace and telemedicine applications [1].

In the herein presented preclinical study, a 5G based data transmission of ultrasound, video and audio source between a moving point of care (ambulance car $=$ first responder) and hospital (stationary unit $=$ medical expert) is evaluated.

The 5G network uses a carrier frequency (C-Band) of $3.41 \mathrm{GHz}$ and bandwidth of $40 \mathrm{MHz}$. Both the base transceiver station (BTS) and the unified user equipment (UE) modem consist of 8 antennas. Subcarrier spacing is $30 \mathrm{kHz}$.

Ultrasound examination was performed by Clarius C3 HD (2-6 MHz) multipurpose scanner (Clarius Mobile Health Corp., Vancouver, Canada). For video and audio transmission, an IP-based pan tilt zoom (PTZ) camera was used (Hikvision DS-2DE2A404IWDE3/W, Hangzhou, China).

\section{Results}

The ultrasound system was successfully connected to the 5G modem and processing system which was installed in a regular vehicle (moving point of care). The remote hospital PC (stationary unit) used to receive and read the ultrasound image data was integrated to the base transceiver station. Through the $5 \mathrm{G}$ network, the ultrasound system has been used to transmit image data to the remote hospital PC (figure 1).

Throughput of total transmitted data through the 5G network between the ultrasound scanner at UE side and the remote hospital PC at BTS side revealed a peak of $6 \mathrm{Mbps}$ (average $4 \mathrm{Mbps}$ ).

The transmission control protocol (TCP), representing the control signalling, showed a peak throughput of $650 \mathrm{Kbps}$ and was transmitted with interruptions. The user datagram protocol (UDP) transmitted data without interruptions, representing the image/data stream from the ultrasound system to the remote hospital PC. Peak UDP throughput was $450 \mathrm{Kbps}$ (average $240 \mathrm{Kbps}$ ). Similar is expected for video and audio data transmission, however, final results are pending.

Conclusion

Preliminary testing of the $5 \mathrm{G}$ based data transmission has been performed successfully. Data throughput requirements for e-health use cases were achieved. The ultrasound image/data has been streamed to the remote hospital PC at BTS side through the 5G network. Logfiles were saved for further analysis.

\section{References}

[1] Jell A, Vogel T, Ostler D, Marahrens N, Wilhelm D, Samm N, Eichinger J, Weigel W, Feussner H, Friess H, Kranzfelder M (2019) 5th-Generation Mobile Communication: Data Highway for Surgery 4.0. Surg Technol Int. 10(35):36-42. 


\section{Automatic facial nerve segmentation from CT using deep learning}

B. S. Jeoun ${ }^{1}$, S. J. Lee ${ }^{2}$, M. H. Choi ${ }^{2}$, J. Y. Yoo ${ }^{2}$, T. H. Yong ${ }^{2}$, S. E. Choi ${ }^{2}$, S. Y. Chun ${ }^{1}$, S. Yang ${ }^{2}$, J. Kim ${ }^{1}$, W. J. Yi ${ }^{3,2}$

${ }^{1}$ Seoul National University, Interdisciplinary Program for Bioengineering, Graduate School of Engineering, Seoul, South Korea ${ }^{2}$ Seoul National University, Department of Biomedical Radiation Sciences, Graduate School of Convergence Science and Technology, Seoul, South Korea ${ }^{3}$ Seoul National University, Department of Oral and Maxillofacial Radiology, School of Dentistry and Dental Research Institute, Seoul, South Korea

Keywords Facial Nerve, Segmentation, CT, ENT

\section{Purpose}

Accurate facial nerve segmentation is considered to be important to avoid any physical damages or paralysis from a patient's face during mastoidectomy for cochlear implantation surgery [1]. Several methods based on conventional approaches have been proposed to perform this segmentation task. Yet, there hasn't been any attempts to utilize deep learning which is the popular approach in many study fields recently. In this work, we studied automatic facial nerve segmentation using various deep learning networks.

\section{Methods}

${ }^{2}$ D U-net, 2D SegNet, 2D Dense U-net, and 3D U-net were utilized as deep learning networks which are commonly used deep learning neural networks for anatomical structure segmentation in the medical imaging fields, such as mammography, MRI, CBCT/CT, etc. The CT data were acquired from 114 subjects and the dataset was split into 91 for train, 23 for test respectively. To obtain label images, a manual segmentation task has done by otolaryngology experts. The resolution of data was $512 \times 512 \times 90$ with $0.6 \mathrm{~mm}$ of voxel spacing. For $2 \mathrm{D}$ networks, each slice of CT data was vertically flipped and randomly rotated in the range of 10 degrees with width-height shifting. For 3D U-net, the data were cropped into $256 \times 256$ due to the memory issue and computational cost. Then zero-padding was implemented to train the network with constant input volume size $256 \times 256 \times 48$ to cover the whole length of the facial nerve which varies from 20 to 45 . We trained networks by using Adam optimizer, learning rate of 0.00025 by reducing on plateau every 25 epoch in 300 epochs with dice coefficient loss. The dice coefficient score was calculated to evaluate each network"s prediction result.

\section{Results}

Table 1 shows the quantitative evaluation result of each network's performance. Even if, 3D U-net has the limitations of high computational cost and memory efficiency, it achieved the best dice coefficient score compared to other 2D networks. Especially, 3D U-net outperformed preserving the continuous feature of facial nerve's canal-like anatomical structure in the perspective of learning spatial information. While, Dense U-net, the modified U-net with dense connections, showed a nearly similar dice coefficient score to 3D U-net, it still had some false positives like 2D U-net and 2D SegNet.

\section{Conclusion}

This work showed the possibility and limitations of automatic facial nerve segmentation using deep learning. In the perspective of its

Table 1 The quantitative evaluation result of each network (Dice Coefficient Score; DSC)

\begin{tabular}{lllll}
\hline Network & 2D U-net & 2D SegNet & 2D Dense U-net & 3D U-net \\
\hline DSC & $0.61 \pm 0.37$ & $0.6 \pm 0.36$ & $0.68 \pm 0.21$ & $0.71 \pm 0.18$ \\
\hline
\end{tabular}

possibility, it is optimistic that the facial nerve segmentation can be done by deep learning which can less labor-intensive and time-consuming annotation task. On the other hand, it is still challenging to achieve accurate facial nerve segmentation result without any disconnections or false positives that can be utilized in clinical application. In future work, we will study advanced and efficient deep learning neural networks that can be applied in real clinical practice. Acknowledgements

This work was supported by the Korea Medical Device Development Fund grant funded by the Korea government (the Ministry of Science and ICT, the Ministry of Trade, Industry and Energy, the Ministry of Health \& Welfare, the Ministry of Food and Drug Safety) (Project Number: 202012E09).

\section{References}

[1] Lu P, Barazzetti L, Chandran V, Gavaghan K, Weber S, Gerber N, Reyes M. (2018) Highly Accurate Facial Nerve Segmentation Refinement From CBCT/CT Imaging Using a Super-Resolution Classification Approach. IEEE Trans Biomed Eng. 2018 Jan;65(1):178-188

\section{Entropy guided refinement strategy for knee cartilage segmentation: data from the osteoarthritis initiative}

\author{
Z. $\mathrm{Li}^{1}$, G. Zheng ${ }^{1}$ \\ ${ }^{1}$ Shanghai Jiaotong University, School of Biomedical Engineering, \\ Institute of Medical Robotics, Shanghai, China
}

Keywords Medical image segmentation, Knee cartilage, Deep learning, Fully supervised segmentation

\section{Purpose}

Knee osteoarthritis (OA) is one of the leading causes of musculoskeletal functional disability and its real burden has been underestimated. Magnetic resonance imaging (MRI), serving as a three-dimensional, noninvasive assessment of cartilage structure, is widely used in OA diagnosis. And many clinical applications, such as quantitative analysis of knee cartilage, requiring efficient segmentation of knee cartilage. To get objective, reproducible clinical analysis, an accurate and high-quality cartilage segmentation from MRI is crucial. Knee cartilages usually have low contrast with surrounding tissues in magnetic resonance imaging. Manual segmentation of the knee joint is tedious, subjective, and labor-intensive. In recent years, with the development of deep learning, knee cartilage segmentation has made great progress. Previous deep learning-based method either integrate Statistical Shape Model (SSM) which need extra prior knowledge or limit in performance. Thus, the objective of this study is to develop a refined knee cartilage segmentation strategy to get highquality knee cartilage segmentation.

\section{Methods}

As shown in Fig. 1, we propose a two-stage knee cartilage segmentation method. It consists of a coarse segmentation stage and an entropy guided refinement stage. Both stages use 3D-Unet with deep supervision as the segmentation network. In the first stage, the network takes original MRI data as input and output probability maps and labels of femur, tibia, femoral cartilage and tibial cartilage. Then, in the second stage, the bone distance map is calculated based on labels of femur and tibia generated in stage one. Bone and cartilage entropy maps are calculated based on probability maps of femur, tibia, femoral cartilage and tibial cartilage generated in the second stage. Entropy maps encode uncertainty information which could guide the network to pay more attention to the boundary of knee 


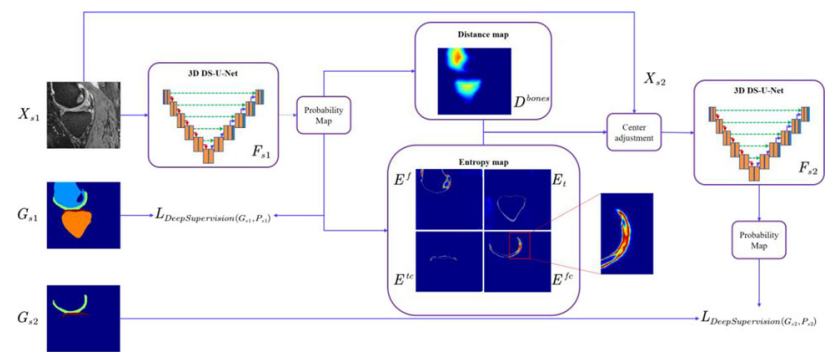

Fig. 1 A schematic illustration of the two-stage deep learning-based method for knee cartilage segmentation is shown

Table 1 Quantitative results of two-fold cross-validation studies for the dataset of OAI-ZIB

\begin{tabular}{lllll}
\hline Method & Parts & DSC $(\%)$ & $\begin{array}{l}\text { ASSD } \\
(\mathrm{mm})\end{array}$ & HD (mm) \\
\hline $\begin{array}{l}\text { Ambellan et al. } \\
{[1]}\end{array}$ & $\begin{array}{c}\text { Femoral } \\
\text { cartilage }\end{array}$ & $\mathbf{8 9 . 9} \pm \mathbf{3 . 6 0}$ & $0.16 \pm 0.07$ & $5.35 \pm 2.50$ \\
& $\begin{array}{c}\text { Tibial cartilage } \\
\text { Ours }\end{array}$ & $85.6 \pm 4.54$ & $0.23 \pm 0.12$ & $6.35 \pm 4.36$ \\
& $\begin{array}{c}\text { Femoral } \\
\text { cartilage }\end{array}$ & $89.8 \pm 2.49$ & $\mathbf{0 . 1 6} \pm \mathbf{0 . 0 5}$ & $\mathbf{5 . 2 2} \pm \mathbf{2 . 4 7}$ \\
& $\begin{array}{c}\text { Tibial cartilage } \\
\text { 86.4 } \pm \mathbf{4 . 1 2}\end{array}$ & $\mathbf{0 . 2 0} \pm \mathbf{0 . 0 9}$ & $\mathbf{4 . 7 0} \pm \mathbf{2 . 1 2}$ \\
\hline
\end{tabular}

cartilages. Finally, entropy maps, distance map and original MRI data are concatenated and feed as the input to the network at stage two, and output refined cartilages segmentation labels. Our method was evaluated on the publicly available dataset, namely OAI-ZIB dataset [1] with femur, tibia, femoral cartilage and tibial cartilage manually segmented, and it contains 507 objects.

Results

Two-fold cross-validation studies are performed for the dataset of OAI-ZIB, and the quantitative results are presented in Table 1. Our method also outperforms the state-of-the-art knee cartilage segmentation method [1] with an improvement of DSC of $0.4 \%$, ASSD of $0.02 \mathrm{~mm}$ and HD of $0.89 \mathrm{~mm}$. In femoral cartilage segmentation, our method achieves DSC of $89.8 \%$, ASSD of $0.16 \mathrm{~mm}$, HD of $5.22 \mathrm{~m}$. And in tibial cartilage segmentation, our method achieves DSC of $86.4 \%$, ASSD of $0.20 \mathrm{~mm}$, HD of $4.70 \mathrm{~mm}$.

Conclusion

In summary, we propose a two-stage entropy guided knee cartilage segmentation method. Our method consists of coarse segmentation and refinement segmentation stages and without the need for additional prior knowledge. In the refinement segmentation stage, the distance map, entropy maps and original MRI data are concatenated to the input. The entropy maps encode the uncertainty information to force the network to pay more attention to the uncertainty area. The distance map encodes more spatial information into the next stage. Our result has shown that the present method achieves good refinement of uncertainty area and get better results than the state-of-the-art method on the OAI-ZIB dataset.

\section{References}

(1) Ambellan F, Tack A, Ehlke M, Zachow S (2019) Automated segmentation of knee bone and cartilage combining statistical shape knowledge and convolutional neural networks: Data from the osteoarthritis initiative. Medical image analysis 52, 109-118
Intestine segmentation combining watershed transformation and machine learning-based distance map estimation

H. Oda ${ }^{1}$, Y. Hayashi ${ }^{2}$, T. Kitasaka ${ }^{3}$, Y. Tamada ${ }^{4}$, A. Takimoto ${ }^{1}$, A. Hinoki $^{1}$, H. Uchida ${ }^{1}$, K. Suzuki ${ }^{1}$, H. Itoh ${ }^{2}$, M. Oda ${ }^{5}$, K. Mori $^{2,6,7}$

${ }^{1}$ Nagoya University, Graduate School of Medicine, Nagoya, Japan

${ }^{2}$ Nagoya University, Graduate School of Informatics, Nagoya, Japan

${ }^{3}$ Aichi Institute of Technology, School of Information Science, Toyota, Japan ${ }^{4}$ Nagoya University, School of Medicine, Nagoya, Japan ${ }^{5}$ Nagoya University, Information and Communications, Nagoya, Japan ${ }^{6}$ Nagoya University, Information Technology Center, Nagoya, Japan ${ }^{7}$ National Institute of Informatics, Research Center for Medical Bigdata, Tokyo, Japan

Keywords small bowel, graphical representation, intestine reconstruction, weak annotation

\section{Purpose}

Intestine (including small intestines) segmentation is desired for diagnosis assistance of the ileus and the intestinal obstruction. There are very few work $[1,2]$ for intestine segmentation methods whose segmentation target is the small intestine. Oda, et al. [1] estimated distance maps on the intestine regions, which represent distances from outside the intestines. Regions in the intestine generated by Watershed transformations on the distance maps are connected and represented as graphs. Those graphical representations allow us to find the longest paths for each intestine's part which is enlarged by contents. This scheme prevents the generation of incorrect contacts between the intestine's different parts, by choosing a high threshold for the Watershed transformation on the distance maps. However, segmentation results become thinner, which cover only around the intestine's centerlines. The intestinal sections tend to be divided at the intestines' sharp curves, even if two sections are apparently continuing for human eyes. Since the small intestines are complicatedly winding, it may not be proper to connect multiple intestinal sections only by examining the minimum distance between their endpoints. We introduce a dual-threshold scheme for the Watershed transformation, which allows us to (1) obtain segmentation results that sufficiently cover intestine regions and (2) check whether two neighboring sections are continuing or not.

Methods

\section{Overview}

Our proposed method performs the segmentation of the intestines from CT volumes. Input is a CT volume. The distance map is estimated on the CT volume, which has high on the centerlines, and low on the intestines' peripheral parts. Using two thresholds for the Watershed transformation, the distance map is converted to two graphs. The higher threshold generates the graphs following major parts of the intestines, in which incorrect contacts are prevented. The low threshold allows us to obtain regions including peripheral parts, and those regions tend to be connected along sharp curves. The output intestine segmentation results are generated by merging the graphs from the higher and the lower thresholds.

Estimation of distance maps

Distance maps are estimated by using the method proposed by Oda, et al. [1]. We introduce a weak-annotation scheme, which requires the intestine labels only on several axial slices for each CT volume in the training dataset. The intestine labels are converted to the distance maps. The distance maps have high values (1.0) in the centerlines, and low values (0.0) in intestine walls or outside the intestines. The 3D U-Net is trained by utilizing pairs of the input CT volumes and the distance maps generated as above. For the testing dataset, distance maps are estimated by the trained 3D U-Net.

Generation of "intestinal segments" 


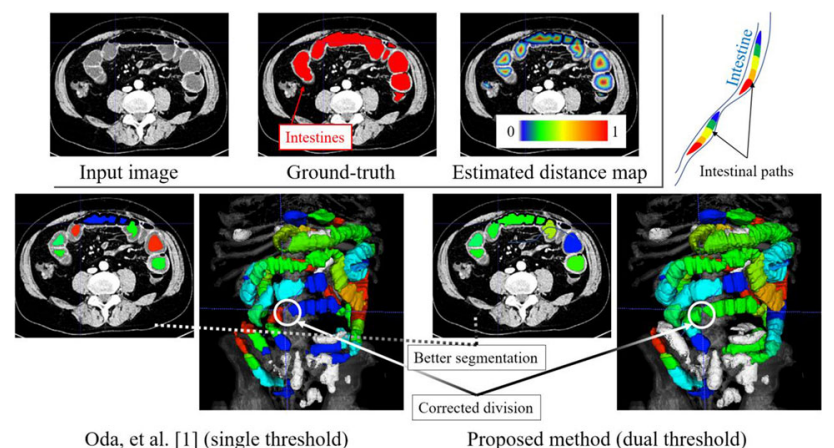

Fig. 1 Segmentation results. Compared to Oda, et al. [1], intestine regions were sufficiently segmented. False division was corrected

We introduce two threshold values $s$ and $t(0<s<t<1)$. The Watershed transformation is utilized for each threshold on the distance maps. Local maxima of the distance maps are used as seeds. Generated regions by the Watershed transformation are called "low/ high-threshold intestinal segments." The high-threshold intestinal segments are thinner than actual intestine regions, and they do not include incorrect contacts between the intestine's different parts. Although the low-threshold intestinal segments sufficiently cover the intestine regions, they often contain incorrect contacts running through the intestine walls.

Representation of intestinal segments' connections as graphs

Connections between the high-threshold intestinal segments are represented as graphs. The graphical analysis allows us to obtain the intestine segments' sequences (called "intestinal paths"). Since the high-threshold intestinal segments are used here, the intestine paths are covering only the intestines' thick parts.

Those intestinal paths are often incorrectly divided at curves of the intestines. Two neighboring intestinal paths are merged into one graph when two intestinal paths are apparent to be connected by the following rule: When intestinal segments of two endpoints of intestinal paths are adjacent (the intestinal segments' shortest distance is less than $d[\mathrm{~mm}]$ ), their corresponding low-threshold intestinal segments are checked. If those low-threshold intestinal segments are touching, two intestinal paths are connected.

Segmentation of intestines

The low-threshold intestinal segments whose corresponding nodes are connected as the intestinal paths are regarded as intestine segmentation results. Other low-threshold intestinal segments are regarded as false positive regions.

\section{Results}

Four-fold cross-validation across 19 patients was conducted. A trained medical student manually traced the intestine regions on 7-10 axial slices for each CT volume as the ground-truth. Parameters were set as: $s=0.01, t=0.2$, and $d=50 \mathrm{~mm}$.

The performances are compared to Oda, et al. [1] which utilizes only one threshold $t$. The Dice score was improved from 0.491 to 0.672. Furthermore, our dual-threshold scheme joined many disconnections. The average number of intestinal paths per CT volume was decreased from 7.9 to 6.2 by joining 1.7 disconnections per CT volume on average. Note that clinicians have not manually confirmed that all of those joinings are correct. Figure 1 shows an example of segmentation results covering entire intestinal regions with connecting incorrectly-divided intestinal paths.

Conclusion

An intestine segmentation method was proposed. The dual-threshold scheme allowed us to (1) obtain segmentation results that are sufficiently covering intestine regions and (2) check whether two neighbouring sections are continuing or not. Future work includes the improvement of network architectures for more accurate segmentation.

\section{Acknowledgements}

Parts of this work were supported by the Hori Sciences and Arts Foundation, MEXT/JSPS KAKENHI (17H00867, 17K20099, 26108006, and 26560255), JSPS Bilateral Joint Research Project, and AMED (JP191k1010036, JP201k1010036).

\section{References}

[1] Oda H, Hayashi Y, Kitasaka T, Tamada Y, Takimoto A, Hinoki A, Uchida H, Suzuki K, Itoh H, Oda M, Mori K (2021) Intestinal region reconstruction of ileus cases from 3D CT images based on graphical representation and its visualization. SPIE Medical Imaging 2021, Computer-Aided Diagnosis: 11597-56

[2] Shin S, Lee S, Elton D, Gulley J, Summers R (2020) Deep Small Bowel Segmentation with Cylindrical Topological Constraints. International Conference on Medical Image Computing and Computer-Assisted Intervention (MICCAI) 2020: LNCS 12264: 207-215

\section{Automatic shoulder bone segmentation from CT arthrograms based on deep learning}

H. Hess ${ }^{1}$, M. A. Zumstein ${ }^{2,3}$, L. Dommer ${ }^{2,3}$, M. Schär ${ }^{2}$, A. Hayoz ${ }^{2,3}$, G. Zeng ${ }^{1}$, A. Ruckli ${ }^{1}$, J. Burger ${ }^{1}$, N. Gerber ${ }^{1}$, K. Gerber ${ }^{1}$

${ }^{1}$ University of Bern, SITEM, Bern, Switzerland ${ }^{2}$ University of Bern, Department of Orthopaedic Surgery and Traumatology, Bern,

Switzerland ${ }^{3}$ Orthopaedics Sonnenhof, Shoulder, Elbow \& Orthopaedic Sports Medicine, Bern, Switzerland

Keywords Convolutional neural network, Segmentation, CT Arthrogram, Shoulder

\section{Purpose}

The shoulder has the largest range of motion of the major joints in the human musculoskeletal system. This high range of motion and mechanical stability is provided by an arrangement of bony and softtissue structures. Differences in the morphology of the scapula result in different biomechanical strains which may predispose a patient to develop certain pathologies or result in worse outcomes of different surgeries. Therefore, morphology analysis has become a crucial tool for clinicians. Novel morphology analysis requires a patient-specific $3 \mathrm{D}$ model which is generated from the semantic segmentation of a medical image data of the patient. However, manual segmentation is time-consuming which hinders the technology to be even more widely integrated into the clinical process. Several techniques are investigated for automatic segmentation of various body-tissues in magnetic resonance images (MRI) or computer tomography (CT) images. Due to the different Hounsfield units of bones and muscles, basic threshold algorithms can be used to segment bones in CT images. However, the different bones can not be distinguished, and contrast agent and image inhomogeneity can reduce the segmentation accuracy. More advanced segmentation techniques, such as atlasbased or statistical shape model-based segmentation techniques, reduce these problems. However, recently the highest segmentation accuracies are achieved with convolutional neural networks (CNN). Segmenting the scapular and humeral bones in shoulder CT arthrograms exhibits multiple additional challenges. The thin structure of the scapular bone shows high variance in shape which aggravates an accurate segmentation. Taghizadeh et al. present in [1] a statistical shape modeling-based algorithm for bone segmentation in non-contrast shoulder CT images. However, in CT arthrograms, which are 

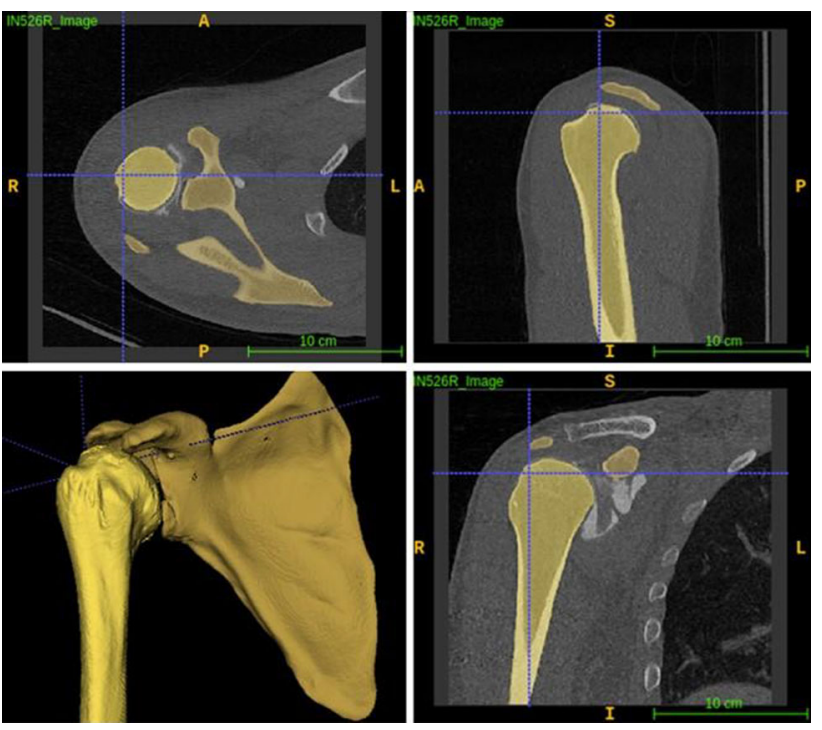

Fig. 1 Example of the automatic scapula and humerus segmentation in a CT Angiogram with an average segmentation accuracy

acquired for the diagnosis of different shoulder pathologies, the contrast agent surrounds the shoulder joint and makes accurate segmentation with a threshold algorithm impossible. The purpose of this study was to develop a CNN-algorithm for accurate bone segmentation of the shoulder in CT arthrograms and evaluate its performance. Methods

Image Acquisition

Shoulder CT arthrograms from 64 patients were acquired during the clinical routine with different CT scanners (Siemens, Toshiba) at the University Hospital of Bern and were used for this study with the approval of the Ethics Committee. Manual Segmentation was performed by clinical experts and used for training the algorithm and as ground truth. All CT images were acquired in the lateral direction with a consistent in-plane resolution of $0.4 \times 0.4 \mathrm{~mm}$ and $512 \times 512$ pixels. The images were created with different Kernels and showed a high variety of quality. The slice thicknesses were between 0.4 and $0.6 \mathrm{~mm}$ and the volumes consisted of various numbers of planes reaching from 160 to 550 (Fig. 1).

Algorithm implementation details

For segmentation, a standard U-Net [2] was applied. The network was trained to segment the scapular and humeral bone separately. An average of the cross-entropy loss and the dice loss was used as loss function during training. Prior to the U-Net, several preprocessing steps were applied. The pixel values were normalized to be around zero with a standard deviation of one and the volumes were interpolated to isotropic volumes with a pixel size of $0.4 \mathrm{~mm}$ in each direction and cropped or extended to volumes with the size 512 in each direction. Data augmentation methods including random zoom and shear were applied during the training process to prevent the network from overfitting. The U-Net was separately trained along all three planes of around 20 epochs. Random initialization was used for the axial plane. For the coronal and sagittal plane, the weights from the axial plane were used for refinement.

Algorithm training and evaluation

Training and predictions were done on a PC (Core i9-9900 K CPU, 64 GB) with two GPUs (GeForce RTX 2080 Ti). Predictions were done on unseen samples in a fivefold cross-validation process. The predictions of all three planes were averaged for final segmentation. The final segmentation was created by isolation of the largest connected component of each label (in 3D) and cropping of all outliers.

\section{Results}

In a fivefold cross-validation process, all CT-Volumes of the 64 patients were segmented by the presented algorithm. The automatic segmentations are compared to the manual segmentations. The segmentations of the humerus show an average Dice coefficient (DC) of 0.988 an average surface distance of $0.21 \mathrm{~mm}$ and a Hausdorff-Distance (HD) of $3.18 \mathrm{~mm}$ while the segmentations of the scapula show an average Dice coefficient of 0.976 an average surface distance of $0.18 \mathrm{~mm}$ and a Hausdorff-Distance of $5.41 \mathrm{~mm}$.

\section{Conclusion}

The CNN-algorithm presented in this study segment the scapular and the humeral bones in shoulder CT arthrograms with high accuracy. The algorithm is capable to segment very thin structures of the scapula despite this bone region shows a high variety in shape. Furthermore, in the majority of the CT arthrograms, the algorithm accurately distinguished between contrast agent and bone and achieved high segmentation accuracies even in the region at the joint, where the contrast agent is close to the bones and shows similar voxel intensities. Therefore, the study results show that automatic segmentation of the shoulder bones with high accuracy is feasible even in CT arthrograms from different CT scanners. This automated process may allow the use of 3D patient data to be more widely integrated into the clinical process for shoulder diagnosis and surgical planning in the future.

\section{References}

[1] Taghizadeh E, Terrier A, Becce F, Farron A, Büchler P (2019) Automated CT bone segmentation using statistical shape modelling and local template matching. Computer methods in biomechanics and biomedical engineering, vol. 22, no. 16, pp. 1303-1310, 2019, https://doi.org/10.1080/10255842. 2019.1661391.

[2] Ronneberger O, Fischer P, Brox T (2015) U-Net: Convolutional Networks for Biomedical Image Segmentation. In: Navab N., Hornegger J., Wells W., Frangi A. (eds) Medical Image Computing and Computer-Assisted Intervention-MICCAI 2015. Lecture Notes in Computer Science, vol 9351. Springer, Cham. https://doi.org/10.1007/978-3-319-24574-4_28

\section{Finite element model validation for the presurgical treatment planning of mandibular angle fractures}

M. Maintz ${ }^{1,2}$, S. Herrmann ${ }^{3}$, N. Sharma ${ }^{2,4}$, F. M. Thieringer ${ }^{2,4}$

${ }^{1}$ University of Applied Sciences and Arts Northwestern Switzerland, Institute for Medical Engineering and Medical Informatics, Muttenz, Switzerland ${ }^{2}$ University of Basel, Medical Additive Manufacturing Research Group, Department of Biomedical Engineering, Allschwil, Switzerland ${ }^{3}$ CADFEM Medical GmbH, Grafing bei München, Germany ${ }^{4}$ University Hospital Basel, Oral- and Maxillo-Facial Surgery \& 3D Print Lab, Basel, Switzerland

Keywords Computer-assisted surgery, Additive Manufacturing, Biomechanical Testing, In silico Validation

\section{Purpose}

Among mandible fractures, the mandibular angle is a frequently fractured region. When stabilization is required, open reduction internal fixation (ORIF) is usually performed using miniplate osteosynthesis. Angular fractures are among the most complex mandible fractures to treat due to the common occurrence of complications related to unsuitable fixation, such as infection, injury of 
the teeth' nerve and roots, loosening of the screws, and damage of the implant. The patient-specific mandible geometry, fracture characteristics, bone composition, and biomechanical forces strongly affect the treatment outcome and should be considered when choosing the plating technique $[1,2]$.

Computer-assisted virtual planning tools such as finite element (FE) simulation provide patient-specific solutions in the surgical decision-making process. The use of FE analysis in medical devices, for example, for the in silico validation of implant designs, is becoming increasingly popular. For the successful treatment of defects and fractures of the mandible and to prevent treatment errors, extensive knowledge of the complex biomechanics and time-consuming and expensive quality examinations are required. Due to the increasing availability of patient-specific implant designs and the ability to model the bone geometry with the aid of computed tomography (CT) data of the patient, the FE method can display the biomechanical bone-implant interaction. An accurate biomechanical model can provide valuable information on the implant's suitability if in vitro and in vivo validation is not possible.

In literature, most of the FE models of the human mandible are not validated. Besides, there is no standard method available to create and validate FE analyses of mandible models. This study aims to develop an accurate bone-implant modelling approach by investigating the fixation of a mandibular angle fracture. The FE model's credibility was assessed in a biomechanical test to establish the suitability of the use of FE methods in pre-surgical planning of mandibular fracture treatment.

\section{Methods}

A digital model of the mandible was created by segmenting the mandibular cortical bone of a female patient's CT scan. Two experienced craniomaxillofacial surgeons were involved in selecting and placing the osteosynthesis plate (Medartis AG, Basel, Switzerland) to fixate an unfavourable angle fracture with a high susceptibility for deformation. The implant was designed explicitly for ORIF of mandible angle fractures. From the patient data, Polyamide 12 (PA12) mandibles $(\mathrm{n}=11)$ were additive manufactured using Selective Laser Sintering (SLS) technology. For the transfer of the virtual fracture planning to the biomechanical/experimental setup, patient-specific cutting and drilling guides were additive manufactured using PolyJet printing technology with a photosensitive polymer DM_8505_Grey20 and OBJET 260 Connex (STRATASYS, Rechovot, Israel) to accurately reproduce the placement of the fracture and the implant on the $\mathrm{n}=8$ PA12 mandible models. The remaining mandibles $(\mathrm{n}=3)$ were used to determine suitable material properties for the FE model. Tensile tests on PA12 samples were performed based on the ISO 527:2019 guidelines to determine the elastic and non-linear plastic deformation of the material, which was incorporated in the engineering data of the simulation. The FE model was set up according to the biomechanical bench test. The intact mandible model was analyzed under loading, and suitable material and friction properties were determined to validate the final, fractured model. In the biomechanical tests, a servo-hydraulic testing machine (Walter and Bai AG, Löhningen, Switzerland) applied a linear, uniaxial load to the mandible angle area to allow the creation of a fracture gap at the anterior border of the ramus. The load was applied in discrete $1 \mathrm{~mm}$ steps, and the reaction force was recorded until $5 \mathrm{~mm}$ axial displacement was reached. The stress-induced surface deformation of the mandible and implant was recorded with an optical scanning device (Atos 3 Triple Scan ARAMIS Professional, GOM GmbH, Braunschweig, Germany). The surface deformation and the fracture gap were compared qualitatively and statistically with the FE simulation results in MATLAB R2019b.

\section{Results}

The comparison between the intact experimental and simulated models resulted in a model with the E-Modulus of $1600 \mathrm{MPa}$, a nonlinear plastic deformation for the PA12 mandible according to the

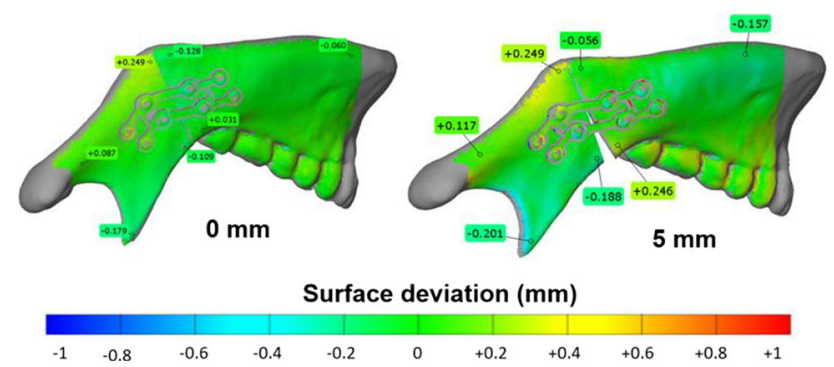

Fig. 1 Mandible and implant surface deviation of experimental 3D scanned surface points and FE model geometry for the axial deformation of $0 \mathrm{~mm}$ and $5 \mathrm{~mm}$

Table 1 The resulting reaction force $(\mathrm{kN})$, and fracture gap $(\mathrm{mm})$ in the biomechanical experiment $(n=8)$ compared to the FE analysis

\begin{tabular}{llllll}
\hline $\begin{array}{l}\text { Axial deformation } \\
(\mathrm{mm})\end{array}$ & \multicolumn{2}{l}{ Reaction Force $(\mathrm{kN})$} & & \multicolumn{2}{l}{ Fracture gap $(\mathrm{mm})$} \\
\cline { 2 - 3 } \cline { 5 - 6 } & Experiment & FEA & & Experiment & FEA \\
\hline 0 & $-0.01 \pm 0.00$ & -0.01 & & $0.01 \pm 0.00$ & 0 \\
1 & $-0.30 \pm 0.07$ & -0.25 & & $0.23 \pm 0.01$ & 0.4 \\
2 & $-0.70 \pm 0.06$ & -0.74 & & $0.64 \pm 0.22$ & 0.89 \\
3 & $-1.02 \pm 0.04$ & -1.10 & & $1.18 \pm 0.06$ & 1.42 \\
4 & $-1.27 \pm 0.04$ & -1.35 & & $1.79 \pm 0.11$ & 2.02 \\
5 & $-1.42 \pm 0.04$ & -1.51 & $2.42 \pm 0.16$ & 2.64 \\
\hline
\end{tabular}

tensile tests, as well as a friction coefficient of 0.2 for the mandiblesteel interface. The additive manufactured cutting and drilling guides effectively reproduced the fracture location and implant positioning. The qualitative analysis of the surface deformation showed a maximum deviation of $0.249 \mathrm{~mm}$ at the inferior border of the proximal segment, as shown in Figure 1. The comparison of the FE analysis and the biomechanical surface deformation of the fixed mandible showed a high degree of agreement in the linear regression (1.045 slope, $0.06 \mathrm{~mm}$ offset, and an $\mathrm{R} 2$ of $99.7 \%(p<0.05))$. The FE model's mean deformation was approximately $-0.11 \mathrm{~mm}$ with a $95 \%$ confidence level of $\left[\begin{array}{ll}0.097 & 0.320\end{array}\right] \mathrm{mm}$. At the maximum axial deformation of $5 \mathrm{~mm}$, the approximate deviation of $0.09 \mathrm{kN}$ and $0.22 \mathrm{~mm}$ was recorded for the reaction force and fracture gap, respectively (Table 1).

\section{Conclusion}

In this study, a biomechanical test setup of a mandible angle fracture model was reproduced in a computational model. The patient mandible model was generated using additive manufacturing of segmented CT data for the biomechanical examination. The FE data regarding surface deformation showed a high level of agreement with the experimental records. This confirms the validity of using FE analysis for the representation of the biomechanical testing outcome. However, to accurately predict the clinical outcome, the model boundary conditions and material properties will have to be adapted to more closely represent the in vivo conditions with the objective of validating the FE results for the human mandible.

\section{References}

[1] Brucoli M, Boffano P, Pezzana A, Benech A, Corre P, Bertin H, Pechalova P, Pavlov N, Petrov P, Tamme T, Kopchak A, Romanova A, Shuminsky E, Dediol E, Tarle M, Konstantinovic VS, Jelovac D, Karagozoglu KH, Forouzanfar T (2019) The 
"European Mandibular Angle" research project: the analysis of complications after unilateral angle fractures. Oral Surgery, Oral Medicine, Oral Pathology and Oral Radiology 128(1), 14-17

[2] Merema BBJ, Kraeima J, Glas HH, Spijkervet FK, Witjes MJ (2021) Patient-specific finite element models of the human mandible: Lack of consensus on current set-ups. Oral Diseases 27(1), 42-51

\section{$\overline{\text { Automatic segmentation and measurement of femoral }}$ head necrosis in CT images}

F. Cao ${ }^{1}$, J. Shen ${ }^{1}$, P. Chen ${ }^{2}$, J. Guo ${ }^{1}$, H. Ge ${ }^{3}$, S. Cai ${ }^{1}$, X. Xiong ${ }^{1}$ ${ }^{1}$ Guangdong University of Technology, School of Automation, Guangzhou, China ${ }^{2} 1$ st Affiliated Hospital Guangzhou University of Chinese Medicine, Guangzhou, China ${ }^{3}$ Guangzhou Hospital of Chinese and Western Medicine, Guangzhou, China

Keywords Automatic CT segmentation, Femoral head necrosis, Necrotic bone segmentation, Necrotic bone measurement

\section{Purpose}

Femoral head-preserving surgery is often performed on a patient with an early stage of osteonecrosis. During surgery, the femoral head is evacuated through a window made at the femoral head-neck junction and replaced by some specific bone materials. The volume of the cavity in a femoral head, where necrotic bone is removed, should be accurately measured. Recent studies propose automatic segmentation methods for segmenting necrotic femoral head based on k-means [1] or deep learning [2]. Overall accuracy of $81.5 \%$ in [1] and $38.9 \%$ in [2] are reported. We propose an automatic CT image segmentation method based on a confidence connected image filter and modified geodesic active contour method. The evaluation of our method shows that a mean of dice coefficients is $82.8 \%$ and a mean segmentation error is $0.9 \mathrm{~cm}^{3}$.

\section{Methods}

In this work, input data is a 3D CT image of a hip joint; output data include the segmentation and the volume size of the necrotic area in a femoral head. Our automatic segmentation method is based on (1) a confidence connected image filter for rough segmentation and (2) the well-known geodesic active contour method for segmentation refinement. The segmentation result of step (1) is used as an initial mask for the step (2) to improve the final segmentation result. The volume size of a necrotic area is calculated by multiplying the voxel size of the 3D CT image by the total number of voxels of the final segmentation result.

\section{Confidence connected image filter}

Confidence connected image filter extracts a connected set of pixels with pixel intensities close to a seed point. Histogram of the CT image is computed and a range of pixel intensities of necrotic bone is defined for automatic selection of a seed point. Then, the mean and standard deviation across a neighborhood of the selected seed point are calculated. They are used with a multiplier factor to define a confidence interval. Pixels with intensity values within the confidence interval are grouped. Finally, in the iteration step, the mean and standard deviation of all the pixels in the previous segmentation are re-calculated to grow the segmented area.

However, segmentation results of confidence connected image filter contain some imperfections and holes. To solve this problem, we use morphological method to fill the holes and obtain a rough segmentation of necrotic bone.

2. Geodesic active contour with a shape-influenced term (GACS)
In a CT image, the boundary of necrotic area in femoral head is not distinct, so we choose the well-known GACS segmentation method which adds the shape and position information of an object to aid the segmentation process. We propose to use the segmentation result of step (1) as an initial contour for GACS method, thereby avoiding manual initialization of level set. Moreover, it provides the surface information of a femoral head to the GACS segmentation process, that adds robustness at the step of the contour evolution. It is because GACS method is based on a prior shape information of a target object to evolve the initial contour globally towards to the shape of the object. Locally, contour evolution is based on image gradients and curvature. Output is a binary mask of the necrosis area of a femoral head.

\section{Results}

The proposed method was evaluated with 6 patients" 3D CT images (size: $512 \times 512 \times 100$, voxel size: $0.782 \mathrm{~mm} \times 0.782 \mathrm{~mm} \times 0.3$ $\mathrm{mm})$ with osteonecrosis. Algorithm was implemented in $\mathrm{C}++$ language and Insight Toolkit (ITK). A CT slice of case 1 and the automatic segmentation result are shown in Figure 1. We evaluated the accuracy of our method with a ground truth (i.e. manual segmentation of an orthopedic surgeon). For each case, we computed dice coefficients using each slice of automatic segmentation and the corresponding slice of the ground truth; then, we calculated a root mean square (RMS) and a standard deviation (STD) using these dice coefficients, as shown in table 1 . The mean of dice coefficients of 6 cases were $82.8 \%$. In addition, a volume size of necrotic bone in femoral head was computed for each case (table 1). We found a mean difference of necrotic bone volumes between our segmentation and the ground truth was $00.9 \mathrm{~cm}^{3}$ (boxplot in Figure 1).

\section{Conclusion}

The main contribution of this work is to propose an automatic CT image segmentation method for osteonecrosis. It is based on confidence connected image filter and geodesic active contour method. The evaluation results show that the proposed method is able to segment femoral head necrosis in a reasonable accuracy which is
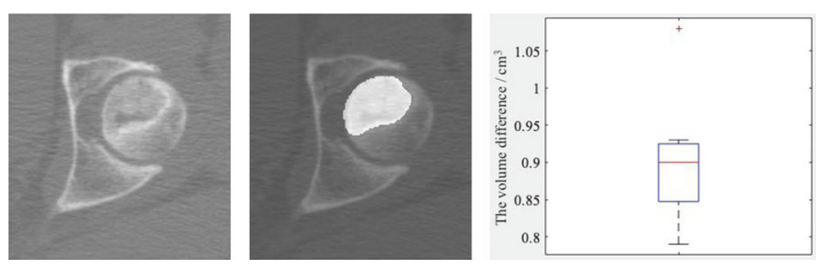

Fig. 1 A slice of CT image of case 1 (left), automatic segmentation of the CT slice (middle) and a boxplot of the volume difference from table 1 (right)

Table 1 Evaluation of the proposed method with the ground truth

\begin{tabular}{|c|c|c|c|c|c|}
\hline \multirow[t]{2}{*}{ Cases } & \multirow[t]{2}{*}{$\begin{array}{l}\text { RMS dice } \\
(\%)\end{array}$} & \multirow[t]{2}{*}{$\begin{array}{l}\text { STD } \\
\text { dice }(\%)\end{array}$} & \multicolumn{2}{|c|}{$\begin{array}{l}\text { Volume of necrotic } \\
\text { bone } / \mathrm{cm}^{3}\end{array}$} & \multirow[t]{2}{*}{$\begin{array}{l}\text { Volume diff./ } \\
\mathrm{cm}^{3}\end{array}$} \\
\hline & & & Automatic & $\begin{array}{l}\text { Ground } \\
\text { truth }\end{array}$ & \\
\hline 1 & 83.6 & 4.45 & 6.87 & 5.96 & 0.91 \\
\hline 2 & 81.7 & 4.58 & 5.03 & 6.11 & 1.08 \\
\hline 3 & 83.2 & 3.76 & 5.28 & 4.35 & 0.93 \\
\hline 4 & 80.3 & 5.61 & 4.06 & 4.93 & 0.87 \\
\hline 5 & 82.9 & 4.37 & 5.62 & 4.78 & 0.84 \\
\hline 6 & 85.2 & 4.12 & 8.31 & 7.52 & 0.79 \\
\hline Mean & 82.8 & 4.48 & 5.86 & 5.61 & 0.9 \\
\hline
\end{tabular}


higher than the accuracy in other studies [1] [2]. The segmentation results can be used for our augmented reality application for femoral head-preserving surgery. Future work will combine CT and MRI image in order to improve the segmentation accuracy and extract more anatomical information in osteonecrosis area.

\section{References}

[1] Zoroofi RA, Sato Y, Nishii T, Sugano N, Yoshikawa H, Tamura $S$ (2004) Automated segmentation of necrotic femoral head from 3D MR data. Comput Med Imaging Graph 28(5): 267-78

[2] Pham DD, Dovletov G, Serong S, Landgraeber S, Jäger M, Pauli J (2020) Multitask-learning for the extraction of avascular necrosis of the femoral head in MRI. Bildverarbeitung für die Medizin: 150-155

\section{Gated convolution network for spine CT inpainting R. Wang ${ }^{1}$, G. Zheng ${ }^{1}$, Y. Zhao ${ }^{1}$ \\ ${ }^{1}$ Shanghai Jiao Tong University, Shanghai, China}

Keywords Partial Convolution, Gated Convolution, Spine CT, Image Inpainting

\section{Purpose}

Numerous factors could lead to partial occlusion of medical images which may affect the accuracy of further image analysis tasks such as semantic segmentation, registration, etc. For example, metallic implants may cause part of the images to deteriorate. The objective of this study is to develop an efficient deep-learning based method to inpaint missing information and to evaluate its performance when applied to spinal CT images with metallic implants.

\section{Methods}

Prior to the partial convolution concept introduced in [1], the inpainting algorithm based on standard convolution usually initialize the holes with some constant placeholder value, and the pixels inside and outside the holes are both regarded as valid pixels. As a result, these methods often produce artifacts so that expensive post-processing needs to be introduced to reduce the impacts of artifacts. To deal with this limitation, the partial convolution applies a masked convolution operation that is normalized to be conditioned only on valid pixels. Then a rule-based mask update strategy that updates the valid locations layer by layer is followed. Partial convolution improves the quality of inpainting on the irregular mask, but it still has remaining issues, such as the mask update mechanism ignoring the receptive field, a varying amount of valid feature points (or pixels) leading to consistent update results, etc. To address the above limitations, the gated convolution is proposed in [2]. Different from the rule-based hard-gating mask updating mechanism in partial convolution, the gated convolution is able to learn the gating mechanism to dynamically control the feature expression of each spatial position of each channel, such as inside or outside masks. Inspired by [2], we propose a gated convolution network $(\mathrm{GCN})$ for spine $\mathrm{CT}$ inpainting. The GCN adopts the framework of the generative adversarial network (GAN), which is composed of a generator and discriminator. Specifically, the generator consists of coarse and refinement networks, and both of them use a simple encoder-decoder structure similar to Unet. While the differences are mainly in the following two points. First, all conventional convolutions are replaced by the gated convolution. Secondly, in image inpainting tasks, it is important that the size of the receptive field is large enough. Therefore, we removed the two downsampling layers in Unet and introduced dilated convolution to expand the model receptive filed without increasing any parameters. Then the combination of dilated convolution and gated convolution, dilated gated convolution, is applied to the GCN. In addition, rich and global contextual information is an important part of discriminant representation for pixel-level visual tasks. However, the convolution operations lead to a local receptive field and thus are not effective for borrowing features from distant spatial locations. To overcome the limitation, we integrate a contextual attention module to capture long-range dependencies. For the discriminator, motivated by global and local GANs, MarkovianGANs, perceptual loss, and spectral-normalized GANs, a spectral-normalized Markovian discriminator is presented to distinguish the inpainting results from the ground truth, thereby prompting the generator to produce more realistic results.

\section{Results}

We evaluate the proposed GCN on the spine CT data from 50 different patients, 10 of which contain real pedicle screw implants. Due to the lack of registered reference image before pedicle screw implantation, the 10 data could not be quantitatively evaluated at the pixel level. Therefore, we adopt subjective experiments to qualitatively evaluate the inpainting image quality of this part of the data. For the other 40 data without implants, we randomly select 20 of them to use the free-form mask generation method to synthesize corresponding masks for training and validation (16 data for training and 4 data for validation). To quantitatively and realistically evaluate the performance of the two inpainting algorithms, for the remaining 20 data, we developed a method to generate simulated pedicle screw implant masks. We respectively adopted subjective and objective methods to evaluate the performance of our method on real data and simulated data. The subjective evaluation metric refers to Mean Opinion Score (MOS), and the objective evaluation metrics include commonly used Mean Absolute Error (MAE), Peak Signal to Noise Ratio (PSNR), and Structural Similarity Index Measure (SSIM). The

Table 1 The quantitative evaluation results of PCN and GCN on the simulated data

\begin{tabular}{|c|c|c|c|c|c|c|}
\hline \multirow{2}{*}{$\begin{array}{l}\text { Case } \\
\text { number }\end{array}$} & \multicolumn{2}{|l|}{ MAE } & \multicolumn{2}{|l|}{ PSNR } & \multicolumn{2}{|l|}{ SSIM } \\
\hline & GCN & PCN & $\mathrm{GCN}$ & $\mathrm{PCN}$ & GCN & $\mathrm{PCN}$ \\
\hline 1 & 3.3167 & 3.5547 & 45.1766 & 44.9548 & 0.9971 & 0.9969 \\
\hline 2 & 2.0114 & 2.4877 & 48.1416 & 46.6749 & 0.9982 & 0.9976 \\
\hline 3 & 1.7505 & 3.6995 & 50.5821 & 45.1820 & 0.9982 & 0.9932 \\
\hline 4 & 1.8512 & 3.4265 & 49.6895 & 44.9026 & 0.9970 & 0.9917 \\
\hline 5 & 2.3212 & 3.2296 & 47.9500 & 45.2147 & 0.9960 & 0.9917 \\
\hline 6 & 2.7930 & 3.1543 & 45.7366 & 44.9263 & 0.9974 & 0.9968 \\
\hline 7 & 3.8326 & 4.8446 & 43.2729 & 41.5865 & 0.9945 & 0.9923 \\
\hline 8 & 1.8237 & 3.4827 & 50.1937 & 45.2708 & 0.9973 & 0.9903 \\
\hline 9 & 6.6554 & 7.3014 & 39.4796 & 38.8284 & 0.9880 & 0.9858 \\
\hline 10 & 2.8412 & 3.4628 & 46.7240 & 45.1855 & 0.9957 & 0.9939 \\
\hline 11 & 1.7596 & 2.7496 & 49.3223 & 45.9686 & 0.9881 & 0.9726 \\
\hline 12 & 3.2994 & 4.9775 & 45.0228 & 42.6120 & 0.9610 & 0.9421 \\
\hline 13 & 2.5129 & 4.4035 & 46.8528 & 42.9081 & 0.9906 & 0.9746 \\
\hline 14 & 2.3651 & 2.6504 & 46.8711 & 45.6282 & 0.9830 & 0.9777 \\
\hline 15 & 1.7867 & 3.9835 & 50.3818 & 44.5360 & 0.9973 & 0.9886 \\
\hline 16 & 3.4360 & 5.3470 & 44.5197 & 41.1009 & 0.9934 & 0.9866 \\
\hline 17 & 2.4982 & 3.0073 & 46.4653 & 44.5323 & 0.9984 & 0.9974 \\
\hline 18 & 2.0163 & 3.1258 & 47.8110 & 43.7487 & 0.9989 & 0.9977 \\
\hline 19 & 3.6754 & 4.0220 & 44.0539 & 42.8442 & 0.9973 & 0.9972 \\
\hline 20 & 1.4526 & 3.7497 & 51.0639 & 44.5460 & 0.9975 & 0.9885 \\
\hline Mean & 2.7000 & 3.8330 & 46.9655 & 44.0576 & 0.9932 & 0.9877 \\
\hline
\end{tabular}




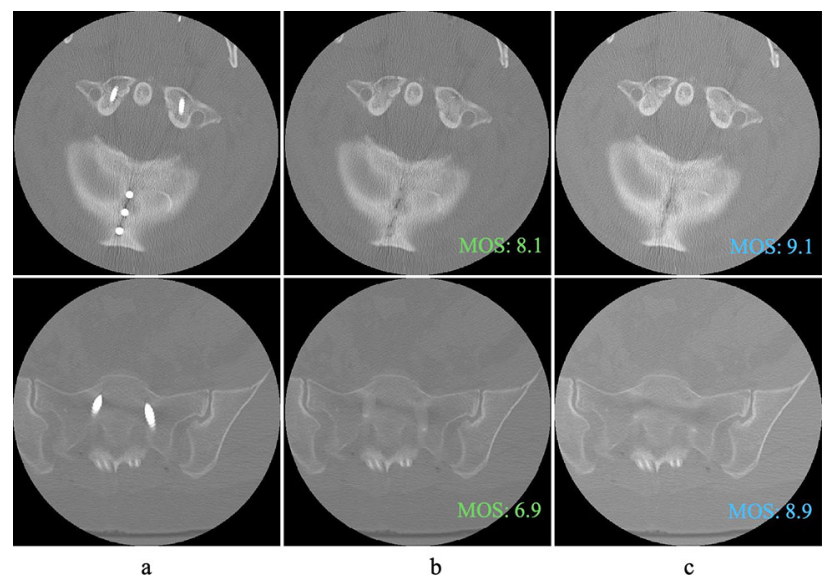

Fig. 1 Example cases of qualitative comparison on real data, where $\mathrm{a}, \mathrm{b}, \mathrm{c}$ represent input with implants, inpainting result of PCN and inpainting result of GCN

evaluation of the inpainting effect consists of two parts: quantitative evaluation of simulated data with reference images and qualitative evaluation of real data without reference images. The quantitative evaluation results of PCN and GCN on the simulated data are shown in Table 1. From a one-to-one quantitative comparison, the MAE of GCN on each data is lower than PCN, while PSNR and SSIM are higher than PCN, which indicates that the superiority of GCN over $\mathrm{PCN}$ in quantitative evaluation metrics is universal. For real implant data, we conducted subjective experiments and used MOS to evaluate the performance of the two models. Finally, the average MOS of GCN and PCN is 8.18 and 7.12, respectively, which indicates that the subjects generally believe that the inpainting results of GCN are more realistic. Figure 1 shows visual examples of image inpainting results based on two algorithms. It can be observed that there are faint traces of implants in the inpainting result of PCN, while the traces are almost imperceptible in the inpainting result of GCN.

\section{Conclusion}

In this study, we propose a gated convolution network for spine CT inpainting. The GCN is based on the generative adversarial network for more realistic prediction, and the gated convolution is used to learn a dynamic feature selection mechanism for each channel and each spatial location. To evaluate the performance of GCN, we respectively carry out a quantitative evaluation on simulated data with reference, and qualitative evaluation on real data without reference. The experimental comparison results with the state-of-the-art method PCN show that the qualitative and quantitative evaluation of GCN has universal advantages. From the final visualization results, we believe that GCN has the potential to be applied to clinical practice.

\section{References}

[1] Liu G, Reda FA, Shih KJ, Wang TC, Tao A, Catanzaro B (2018) Image inpainting for irregular holes using partial convolutions. In Proceedings of the European Conference on Computer Vision (ECCV) (pp. 85-100).

[2] Yu J, Lin Z, Yang J, Shen X, Lu X, Huang TS (2019). Free-form image inpainting with gated convolution. In Proceedings of the IEEE International Conference on Computer Vision (pp. 4471-4480).
3D modeling in hand surgery-a comparison of data quality from multi sliced CT (MSCT) and cone beam CT (CBCT) using different software

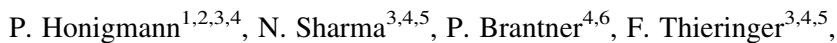
M. Haefeli ${ }^{2,7}$

${ }^{1}$ Kantonsspital Baselland, Hand Surgery, Liestal, Switzerland

${ }^{2}$ Amsterdam UMC, University of Amsterdam, Department of Biomedical Engineering and Physics, Amsterdam Movement Sciences, Amsterdam, Netherlands ${ }^{3}$ Medical Additive Manufacturing, Department of Biomedical Engineering, University Basel, Basel, Switzerland ${ }^{4}$ 3D Print Lab, University Hospital Basel, Basel, Switzerland ${ }^{5}$ University Hospital Basel, Department of Oral and Cranio-Maxillofacial Surgery, Basel, Switzerland ${ }^{6}$ University Hospital Basel, Radiology Department, Basel, Switzerland ${ }^{7}$ Kantonsspital Graubünden, Hand Surgery, Chur, Switzerland

Keywords Cone Beam CT, Multi sliced CT, Segmentation, 3D modeling

\section{Purpose}

A high level of data quality is mandatory for 3D-modelling in Hand Surgery. Used data are Digital Communication in Medicine (DICOM)-images obtained by computed tomography (CT) scans.

The digital three-dimensional (3D) preparation of acquired data is usually in the hands of the radiologist who is a mandatory member of the team offering a 3D-printing service in-hospital.

Usually a multi-detector CT (MDCT)-scanner is available in larger hospitals and sometimes a Magnetic Resonance Imaging (MRI) machine. During the last decade the use of cone-beam computed tomography (CBCT) extended from the main application field of dental imaging to hand- and foot imaging. Recent developments aim for full body cone-beam computed tomography.

\section{Methods}

We compared the quality of the data obtained by MSCT and CBCT using different segmentation software (Mimics, DDS Pro, Horos, DISIOR) to create 3D models for hand surgical purposes. The data were compared using standard registration procedures in GOM Inspect and Mimics.

\section{Results}

There are differences in terms of quality of segmentation between the types of software. DIfferences were below $1 \mathrm{~mm}$. We found that DICOM-data from cone beam CT showed less noise and artifacts compared to multislice CT data. This led to better segmentation results which facilitated 3D modeling, see figures 1 and 2 .

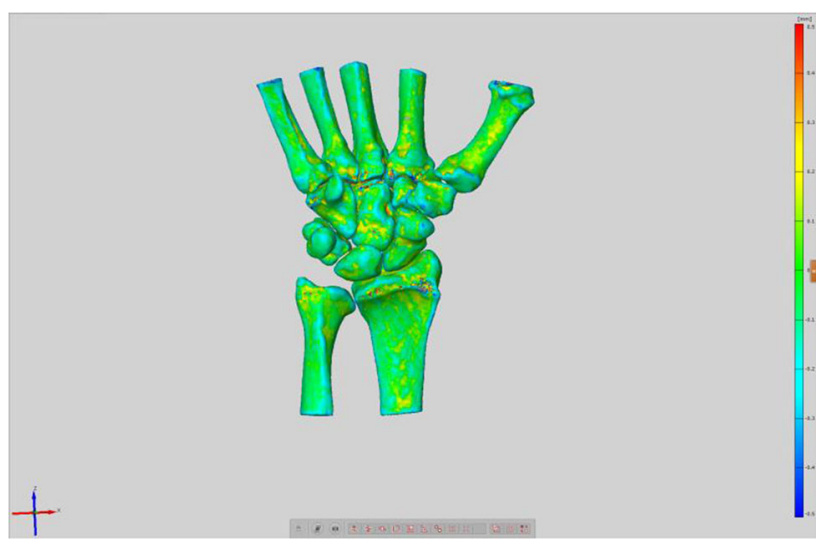

Fig. 1 Palmar-dorsal view of a segmented wrist model comparing of segmentation results of Mimics and DDS of CBCT DICOM-data 


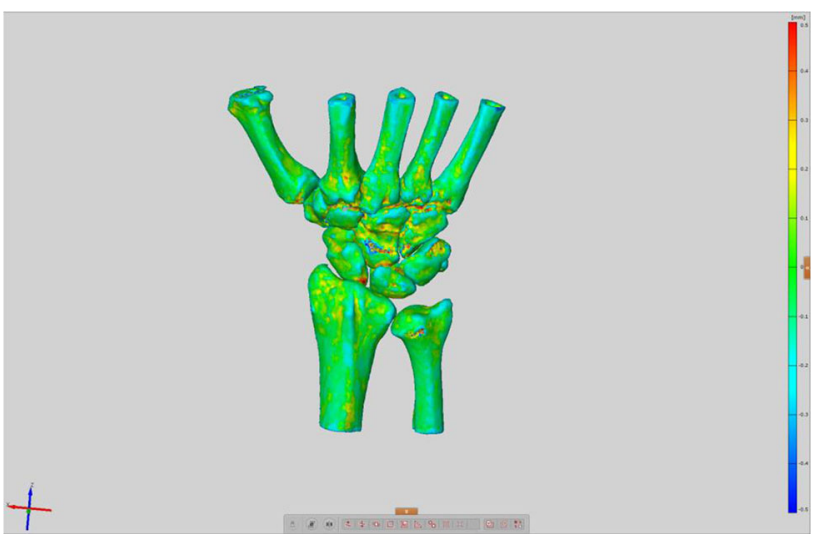

Fig. 2 Dorso-palmar view of a segmented wrist model comparing of segmentation results of Mimics and DDS of CBCT DICOM-data

\section{Conclusion}

DICOM data obtained by CBCT meet the quality requirement of almost all purposes for 3D modeling in Hand Surgery. There need to be an awareness that differences in segmentation procedures throuout the available software on the markers may lead to clinically relevant changes in 3D-modeling for a precision needed for hand surgical purposes.

\section{Computer-aided planning of personalized biopsy of brain tumor based on multi-modality fusion}

\author{
Y. Zhao ${ }^{1}$, G. Zheng ${ }^{1}$ \\ ${ }^{1}$ Shanghai Jiao Tong University, School of Biomedical Engineering, \\ Institute of Medical Robotics, Shanghai, China
}

Keywords Biopsy planning system, Multi-modality image fusion, Semi-automatic segmentation, MITK

\section{Purpose}

The biopsy of brain tumor plays a vital role in the minimally invasive interventional treatment of tumor. The accuracy of the biopsy of the tumor directly determines the therapeutic effect of the subsequent surgery. At present, the clinical biopsy of tumors is generally performed by free-hand under image-guidance. There are still many drawbacks to free-hand biopsy, which limit the clinical use of biopsy technology. The primary research goal of this paper is to study several key technical problems in the process of biopsy surgery, and a computer-aided personalized biopsy planning system of brain tumor is developed to provide the surgeon with more spatial information about the tumor while reducing the fatigue of the surgeon, and assist the surgeon in completing the biopsy operation more conveniently and accurately.

Methods

A personalized biopsy planning system of brain tumor is developed based on the Medical Imaging Interaction Toolkit (MITK), which consists of four parts: multi-modality image registration and fusion module, semi-automatic segmentation module, 3D reconstruction module and plan design of biopsy path module.

For the multi-modality image registration and fusion module, a multi-modality image 3D rigid registration method based on mutual information is designed to register the MR image into the physical space of the CT image. The registered MR image and the CT image are fused with an average weighting method, so that the fused image contains both the tumor and soft tissue information in the MR image and the bone and skin information in the CT image. The multi- modality image registration algorithm is implemented by the Insight Toolkit (ITK). The multi-modality image registration and fusion module is integrated into the biopsy planning system as the "Registration plugin".

For the semi-automatic segmentation module, the graph cut algorithm[1] is used to semi-automatically segment brain tumor in the registered MR image. With the graph cut algorithm, users need to interactively delineate the tumor area and the normal tissue area on the registered MR image, and the algorithm will automatically complete the segmentation calculation of the entire tumor area. The graph cut algorithm is implemented by the Insight Toolkit (ITK). The semi-automatic segmentation module is integrated into the biopsy planning system as the "GraphCut3D plugin".

For the 3D reconstruction module, a reconstruction pipeline based on the marching cubes algorithm[2] is designed. Before the marching cubes algorithm, the image pre-processing operations, such as median filtering and Gaussian filtering, are used to reduce noise in the image. Then the marching cubes algorithm is used to reconstruct the 3D mesh model of the surface skin and tumor. After the surface model is obtained, post-processing operations are used to improve the quality of the model, such as mesh smoothing, normal vector calculation, and connected region analysis. Based on the CT image and the segmented tumor binary image, 3D mesh models of the surface skin and tumor are reconstructed respectively. The reconstruction pipeline is implemented by the Visualization Toolkit (VTK). The 3D reconstruction module is integrated into the biopsy planning system as the "Mesher plugin".

For the plan design of biopsy path module, users can interactively select biopsy target point and needle entry point on the tumor and skin surface to generate the biopsy path, and interactively cut out the skin surface to generate a biopsy guide plate STL model through 3D printing. The biopsy guide can be obtained to assist the surgeon to realize a more accurate and personalized biopsy plan design of brain tumor. The plan design of biopsy path module is integrated into the biopsy planning system as the "Plan Design plugin".

\section{Results}

The multi-modality image registration algorithm is evaluated on the "ACRIN-FMISO-Brain" dataset from "The Cancer Imaging Archive (TCIA)". The Euclidean distance between the anatomical landmarks after registration is calculated to represent the registration error. The graph cut algorithm is evaluated on the "MICCAI BraTS2017" dataset. The dice similarity coefficient (DSC), positive predictive value (PPV) and sensitivity are calculated to represent segmentation accuracy. The mean registration error is $1.3321 \pm 0.3070 \mathrm{~mm}$. The mean DSC is $0.9130 \pm 0.0188$, the PPV is $0.9387 \pm 0.0297$, and the sensitivity is $0.8910 \pm 0.0452$.The results of registration error and segmentation accuracy are shown in Table 1 . With the aid of the biopsy planning system, the surgeon can perform a personalized biopsy plan design for the patient, and the biopsy guide will be generated to assist the surgeon in performing the biopsy operation.

Table 1 The results of registration error and segmentation accuracy

\begin{tabular}{|c|c|c|c|c|}
\hline & \multicolumn{3}{|c|}{ Segmentation accuracy } & \multirow{2}{*}{$\begin{array}{l}\text { Registration error } \\
(\mathrm{mm})\end{array}$} \\
\hline & DSC & PPV & Sensitivity & \\
\hline Mean & 0.913 & 0.9387 & 0.891 & 1.3321 \\
\hline Median & 0.921 & 0.9552 & 0.9077 & 1.1896 \\
\hline Maximum & 0.9404 & 0.9825 & 0.9536 & 2.3478 \\
\hline Minimum & 0.8803 & 0.8838 & 0.8 & 0.5291 \\
\hline Standard deviation & 0.0188 & 0.0297 & 0.0452 & 0.307 \\
\hline
\end{tabular}




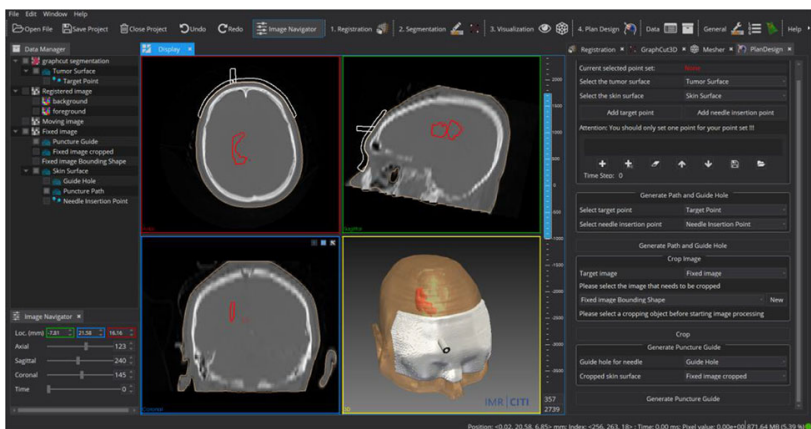

Fig. 1 Interface of the biopsy planning system

The interface of the biopsy planning system is shown in figure 1 . The data management module is located on the left side of the system interface, which is used to manage, display and modify data. The core plugins are located on the right side of the system interface, which realize the functions of registration and fusion, semi-automatic segmentation, 3D reconstruction and planning design respectively. The four windows in the middle of the system interface display twodimensional images of different views and three-dimensional space respectively. Figure 1 shows the result of the biopsy planning. The red tumor model reconstructed from the 3D reconstruction module is segmented from the registered MR image, which is in the same space as the CT skin model. The path pointed by the white guide plate hole is the biopsy path designed by the user. This guide model can be used to assist in biopsy surgery after being printed by 3D printing technology.

\section{Conclusion}

A computer-aided personalized biopsy planning system of brain tumor is developed to help the surgeon design an ideal biopsy path of brain tumor before surgery, thereby reducing the complexity of biopsy surgery, increasing the success rate of subsequent surgery, and reducing the amount of extra trauma and radiation for patients. With the aid of this system, the surgeon can complete the biopsy operation more conveniently and accurately.

\section{References}

[1] Boykov YY, Jolly MP (2001) Interactive graph cuts for optimal boundary \& region segmentation of objects in ND images. In Proceedings eighth IEEE international conference on computer vision. ICCV 2001 (Vol. 1, pp. 105-112). IEEE.

[2] Lorensen WE, Cline HE (1987) Marching cubes: A high resolution 3D surface construction algorithm. ACM siggraph computer graphics, 21(4), 163-169

\section{$\overline{\text { Semantic segmentation for tooth type using DeepLab v3 }}$ with adversarial training}

D. Hayashi ${ }^{1}$, R. Nakayama ${ }^{1}$, A. Hizukuri ${ }^{1}$, K. Murata ${ }^{2}$

${ }^{1}$ Ritsumeikan, Electronic and Computer Engineering, Noji-higashi, Kusatsu, Shiga, Japan ${ }^{2}$ TAKARA TELESYSTEMS Corp.,

Oosakashichuoku, Osaka, Japan

Keywords Semantic Segmentation for Toot, Adversarial Training, Panoramic Dental X-ray Imaging, Computer Aided Diagnosis

\section{Purpose}

Although panoramic dental X-ray imaging is usually used for diagnosis of dental lesion, the spatial resolution of those images is relatively low. Thus, small and obscure lesions are often overlooked.
Therefore, it is desirable to develop a computer-aided diagnosis (CAD) scheme for panoramic dental X-ray images. In the CAD scheme for dental images, it is often necessary to segment tooth for each tooth type.

Semantic segmentation (SS) is one of the labeling methods that associate each pixel of an image with a class label. The SS models have been developed with deep learning approaches such as convolutional neural networks (CNNs). Those SS models are usually trained to minimize the error in an entire image. When applying those SS models to the segmentation of small region such as each tooth, the segmentation accuracy might be low. It is necessary in the segmentation of each tooth to train the SS model with more focus on image features for each tooth type.

There is an adversarial training as a learning method to train Generative Adversarial Network (GAN). In this study, we propose a computerized method for the segmentation of tooth focusing on each tooth type by applying the adversarial training to the learning of a SS model. A novel network was constructed by adding a CNN to the SS model. The added CNN classified ROIs (region of interest) surrounding a tooth extracted from a SS result image and the teacher label image. The SS model and the CNN in the proposed network were learned with the adversarial training.

\section{Methods}

Our database consisted of 161 panoramic dental X-ray images obtained from 161 patients. Label images for each of 32 tooth types were generated by manually annotating each tooth region in the panoramic dental X-ray images. Those images were randomly divided into three datasets. The training, validation, and test datasets included 81,20 , and 60 images, respectively.

In this study, a novel network was constructed by adding a CNN to a SS model for the segmentation of tooth for each tooth type. Figure 1 shows the proposed network. To determine the SS model to use in the proposed network, three different SS models (FCN, U-Net, and DeepLab v3) were employed to segment tooth for tooth type. The model with the highest segmentation accuracy for the validation dataset was then determined as the optimal SS model. Based on the label image, ROIs (region of interest) surrounding each tooth were extracted from the original image, the label image, and the SS result image, respectively. The pair of the original ROI and the label ROI or the SS ROI was inputted to the added CNN. The CNN outputted two classification results. The CNN discriminated the SS ROI pair from the label ROI pair, and also identified the tooth type of the inputted ROI pair. The added CNN was constructed from five layer-blocks consisting of a convolutional layer, a Leaky ReLU, and a batch normalization layer. The SS model was trained with more focus on

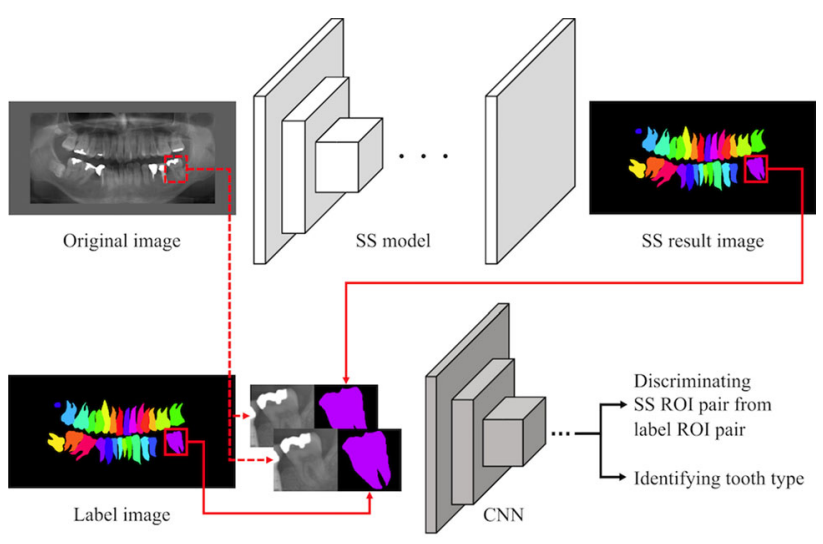

Figure 1: Schematic of the proposed network

Fig. 1 Schematic of the proposed network 
image features for each tooth type by applying the adversarial training to the SS model and the added CNN. In this study, IoU (Intersection over Union) was used as the evaluation index for the segmentation accuracy of tooth for each tooth type.

Results

The mean IoU with DeepLab v3 for the validation dataset was 0.562 , which was significantly improved compared to FCN (0.554, $\mathrm{P}=0.023)$ and U-Net $(0.488, p<0.001)$. Therefore, DeepLab v3 was determined as the optimal SS model in the proposed network. The mean IoU with the proposed network for the test dataset was 0.687, which was greater than that with DeepLab v3 (0.611, $p<0.001)$. Tooth roots with a low contrast to the alveolar bone were more accurately segmented using the proposed network than DeepLab v3.

Conclusion

To train the SS model with more focus on image features for each tooth type, a novel network was constructed by adding a CNN to DeepLab v3. The proposed network with the adversarial training exhibited higher segmentation accuracy of tooth in panoramic dental X-ray images compared to DeepLab v3.

\section{Computer-aided diagnosis method for radiographic bone loss and periodontitis stage based on deep learning: a multi-device study}

S. J. Lee ${ }^{1}$, H. J. Chang ${ }^{2}$, S. Yang ${ }^{1}$, T. H. Yong ${ }^{1}$, J. Kim ${ }^{3}$, W. J. Yi ${ }^{1,2,3}$ ${ }^{1}$ Seoul National University, Department of Biomedical Radiation Sciences, Seoul, South Korea ${ }^{2}$ Seoul National University, Department of Oral and Maxillofacial Radiology, Seoul, South Korea ${ }^{3}$ Seoul National University, Department of Bioengineering, Seoul, South Korea

Keywords Deep learning, Computer-aided diagnosis (CAD), Periodontitis, Radiographic bone loss

\section{Purpose}

Periodontal diseases, including gingivitis and periodontitis, are some of the most common diseases that humankind suffers from. In 2017, the American Academy of Periodontology and the European Federation of Periodontology proposed a new definition and classification criteria for periodontitis based on a staging system [1].

In our previous study, a deep learning hybrid framework was developed to automatically stage periodontitis on dental panoramic radiographs according to the criteria that was proposed at the 2017 World Workshop [2]. In this study, the previously developed framework was improved in order to classify periodontitis into four stages by detecting the number of missing teeth/implants using an additional $\mathrm{CNN}$. A multi-device study was performed to verify the generality of the method.

Methods

\section{Overview}

Figure 1 shows the overall process for a deep learning-based CAD method for measuring the radiographic bone loss (RBL) and determining the periodontitis stage.

\section{Data preparation}

The panoramic radiographs of each patient were acquired with three dental panoramic X-ray machines from three devices. A total of 410 panoramic radiographs of the patients were acquired by using device 1, an orthopantomograph OP 100 (Instumentarium corporation, Tuusula, Finland). A total of 60 panoramic radiographs of the patients for devices 2 and 3 were acquired using PaX-i3D Smart (Vatech, Seoul, Korea) and Point3D Combi (PointNix, Seoul, Korea), respectively. With regard to the panoramic image dataset, 500, 500, and 300 images were used to detect the anatomical structures, such as

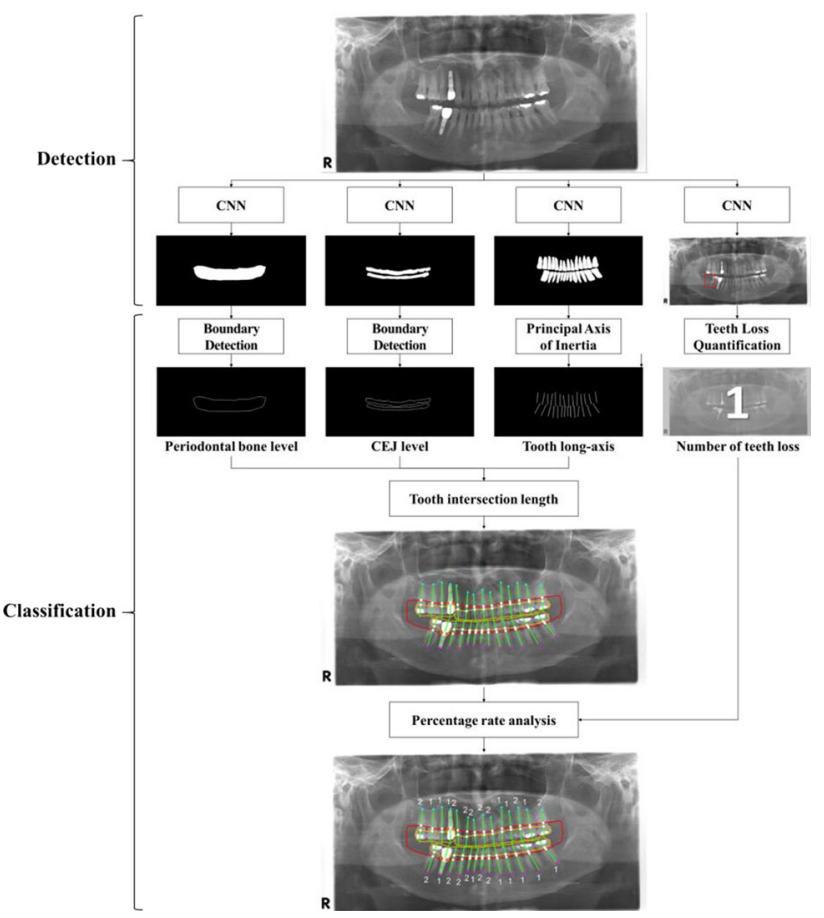

Fig. 1 Overall process for a developed computer-aided diagnosis method for radiographic bone loss and periodontitis stage based on deep learning on dental panoramic radiographs

the periodontal bone level (PBL), cementoenamel junction level (CEJL), and teeth/implants, respectively.

To detect the location of the missing teeth and to quantify their number, the image of the patient with the missing teeth was selected and classified into two types according to the number of missing teeth. The loss of one non-contiguous tooth was classified as type 1 , and loss of two consecutive teeth was classified as type 2. As a result of the selection, 147 and 62 images were used for detecting and quantifying the missing teeth for type 1 and type 2, respectively.

Detection of anatomical structures using CNN

By using a similar procedure in a previous study [2], the PBL was annotated as one simple structure for the whole jaw on the panoramic radiograph; the CEJL of the teeth (the fixture top level of implants) was annotated as one structure that included the crowns of the teeth and implants at the maxilla and the mandible, respectively.

Mask R-CNN was trained for detecting the PBL, CEJL, and teeth/ implants by using the multi-device image dataset. After training the $\mathrm{CNN}$, the segmentation accuracy of each CNN was calculated using the images in the test set. The CNN was implemented by using Python with the Keras and TensorFlow libraries. The CNN was trained by applying the transfer learning method based on the weights that were calculated in the previous study.

After training, the CNNs produced a segmentation mask of the anatomical structures for the input panoramic image. The periodontal bone levels were then detected by extracting the edge of the segmented image (Figure $2 \mathrm{a}-\mathrm{c}$ ). The same process was applied for the detection of the CEJL (Figure 2d-f), teeth, and implants from their segmentation mask (Figure $2 \mathrm{~g}-\mathrm{i}$ ).

\section{Detection of the missing teeth using the CNNs}

Each image was manually labeled by drawing rectangular bounding boxes around the location of the missing teeth with a labeling software for the bounding box detection task named the YOLO mark.

Two modified CNNs from the YOLOv4 networks named CNNv4, and CNNv4-tiny were used for detecting and quantifying the missing 


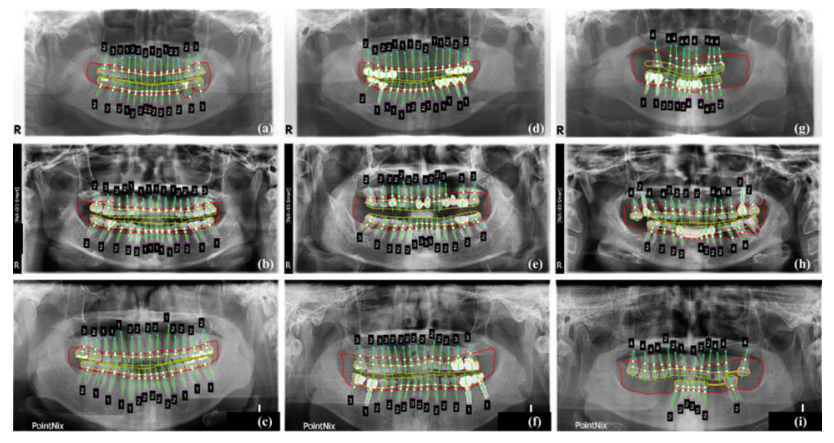

Fig. 2 The stages of the periodontitis for each tooth and implant on the dental panoramic radiographs acquired from multiple devices. The automatic diagnosis results by the developed method on the images. The first, second, and third rows are the images from the device 1 , device 2 , and device 3 , respectively

teeth on the panoramic radiographs. The network was trained on a total of 2000 epochs with a 64-batch size and one- or two-stride sizes. Staging periodontitis by the conventional CAD method

The method of automatically diagnosing the stage of periodontitis was the same as the method that was used in our previously published paper [2]. The RBL of the tooth was automatically classified to identify the stage of periodontitis according to the criteria that was proposed at the 2017 World Workshop [1].

\section{Results}

Detection performance for the anatomical structures and the missing teeth.

The DSC values for Mask R-CNN were 0.96, 0.92, and 0.94, for the detection of the PBL, CEJL, and teeth/implants, respectively.

For the detection of the missing teeth, the precision values for CNNv4-tiny, and CNNv4 were 0.88 , and 0.85 , respectively. The recall values for CNNv4-tiny, and $\mathrm{CNNv} 4$ were 0.85 , and 0.85 , respectively. The $\mathrm{F} 1$-score values for $\mathrm{CNNv} 4$-tiny, and $\mathrm{CNNv} 4$ were 0.87 , and 0.85 , respectively. The mean of the AP values for CNNv4tiny, and CNNv4 were 0.86 , and 0.82 , respectively.

Classification performance for the periodontitis stages.

Figure 2 shows the completely classified stages of the periodontitis for the teeth/implants using the multi-device images.

To evaluate the classification performance of the periodontal bone loss, the mean absolute differences (MAD) between the stages that were classified by the automatic method and the radiologists" diagnoses were compared. These MAD values were $0.26,0.31$, and 0.35 for the radiologists with ten-years, five-years, and three-years of experience, respectively, for the teeth of the whole jaw. The overall MAD between the stages with the automatic method and the radiologists while using all the images was 0.31. For the images from multiple devices, the MAD values were $0.25,0.34$, and 0.35 for device 1 , device 2 , and device 3 , respectively, for the teeth/implants for the whole jaw.

\section{Conclusion}

The developed method used the percentage rate of the periodontal bone loss to automatically and classify the periodontitis into four stages of the whole jaw according to the renewed criteria that was proposed at the 2017 World Workshop [1]. The developed method can help dental professionals to diagnose and monitor periodontitis systematically and precisely on panoramic radiographs. In future investigations, the method must be improved to diagnose the stage of periodontitis while considering both the severity and complexity factors.

\section{References}

[1] Tonetti MS, Greenwell H, Kornman KS (2018) Staging and grading of periodontitis: Framework and proposal of a new classification and case definition. Journal of Clinical Periodontology, 2018. 45 Suppl 20: p. S149-S161

[2] Chang H-J, Lee S-J, Yong T-H, Shin N-Y, Jang B-G, Huh K-H, Lee SS, Heo M-S, Choi S-C, Kim T-I, Yi W-J (2020) Deep Learning Hybrid Method to Automatically Diagnose Periodontal Bone Loss and Stage Periodontitis. Scientific Reports, 2020. 10(1): 7531

\section{Acknowledgements}

This work was supported by the Korea Medical Device Development Fund grant funded by the Korea government (the Ministry of Science and ICT, the Ministry of Trade, Industry and Energy, the Ministry of Health \& Welfare, the Ministry of Food and Drug Safety) (Project Number: 202011B05).

\section{Intraoperative feedback for laparoscopic access with surgical audio guidance}

\author{
M. Spiller ${ }^{1}$, T. Suehn ${ }^{1}$, N. Esmaeili ${ }^{1,2}$, A. Boese $^{1}$, M. Friebe $^{1}$, A. \\ Illanes $^{1}$ \\ ${ }^{1}$ Otto-von-Guericke University, Medical Faculty, INKA-Innovation \\ Laboratory for Image Guided Therapy, Magdeburg, Germany \\ ${ }^{2}$ Technical University Munich, Chair for Computer Aided Medical \\ Procedures and Augmented Reality, Munich, Germany
}

Keywords Laparoscopic Access, Needle Guidance, Audio Sensing, Real-time Feedback

\section{Purpose}

For the laparoscopic access, the artificial creation of a pneumoperitoneum, carbon dioxide must be introduced into the abdominal cavity to create the field of vision and work for the laparoscopic procedure. For the safe generation of the pneumoperitoneum at the beginning of a laparoscopic procedure, the Veress needle is widely used. Currently, there is no imaging modality or other means of providing navigation information for this procedure. The surgeons rely on their sense of touch to guide the Veress needle. Due to this subjective and errorprone technique, the procedure is associated with considerable risks. About $50 \%$ of all laparoscopy complications, like injuries to blood vessels or intra-abdominal organs, are caused when surgical access is created [1]. In addition to the patient's risk and an extended recovery time, this results in considerable additional procedure time and cost.

Alternative sensor-based tools have been proposed for improving the precision and safety of laparoscopic access. Usually, those solutions comprise of a sensor embedded at the tip of the tools leading to direct tissue contact and sterilization issues. Additionally, those tools are mostly complex and expensive and have not been commercially successful.

Proximal audio sensing has demonstrated to be able to differentiate between Veress needle events and detect tissue-layer crossings [2]. This concept uses an audio sensor mounted to the proximal end of a tool to capture information about tool-tissue interactions. Based on this concept, we are developing Surgical Audio Guidance (SURAG). This intraoperative feedback system processes the acquired audio signal and extracts useful guiding information to provide safety-relevant feedback. We proposed and tested an auditory and a visual feedback method. The results show that the information provided is in 
line with the events detected by proximal audio sensing and can enhance the surgeons' perception.

\section{Methods}

The visual feedback is based on the time-domain audio signal and its Continuous Wavelet Transformation (CWT) and is presented in realtime to the surgeon. To evaluate this variant, 40 needle insertions were performed using two types of Veress needles, one for single-use and one for multiple-use. As shown in Figure 1, the insertions were performed by a testing machine (Zwicki, Zwick GmbH \& Co. KG, Ulm) that recorded the axial needle insertion force at $100 \mathrm{~Hz}$, while the needle was inserted into an ex vivo porcine tissue phantom at an insertion velocity of $8 \mathrm{~mm} / \mathrm{s}$. An audio signal was acquired during the insertion at a sampling frequency of $16.000 \mathrm{~Hz}$ using a MEMS microphone sensor placed at the needle's proximal end. The acquisition of force and audio was synchronized using a trigger event visible in both the force and the audio signals. The visual feedback was evaluated by comparing the events observed in the audio and CWT spectrum with the reference force signal.

The auditory feedback was implemented into a small add-on mounted to the proximal end of a Veress Needle. The add-on comprises of a piezoelectric MEMS microphone (PMM-3738-VM1000-R, Vesper, Boston), an nRF52832 microcontroller (Nordic Semiconductor, Trondheim) for digital processing, and a speaker for playing the feedback. Due to the speaker's low mass and damping measures in the add-on housing, it is ensured that potential vibrations caused by the speaker do not interfere with the audio acquisition. To evaluate if the auditory feedback enhances the surgeons' perception of tissuelayer crossings, compared to the existing approach, the add-on has been tested by three domain experts who repeatedly inserted different Veress needles into an ex vivo porcine tissue phantom.

\section{Results}

Figure 2 shows an exemplary comparison of an audio and a force signal and the time-domain visual feedback. Each significant response in the audio and CWT spectrum corresponds to an event triggered by the Veress needle's entry into a major anatomical structure, identifiable by a significant peak in the force signal. For each of these events, a response in the spectrum can be observed. Equal observations have been made for more than $95 \%$ of the insertions independent of the needle's type and the insertion velocity. This indicates that the visual feedback can accurately display tissue-layer crossings during laparoscopic access.

For the auditory feedback, the domain experts invited to test the add-on consistently stated that the feedback was in line with their tactile feedback and enhances their perception of tissue-layer crossings. Furthermore, they indicated that additional feedback might be

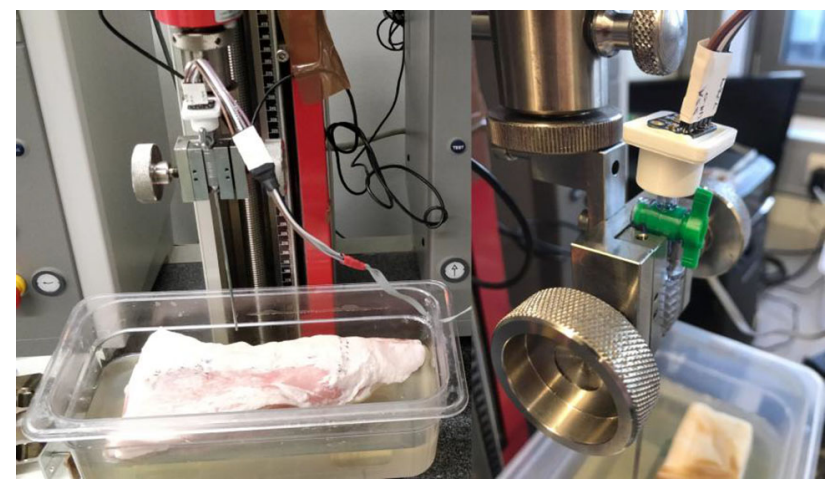

Fig. 1 The visual feedback has been tested using a Zwick testing machine that inserts a Veress needle into an ex vivo porcine tissue phantom. The audio sensor is mounted to the proximal end of the needle
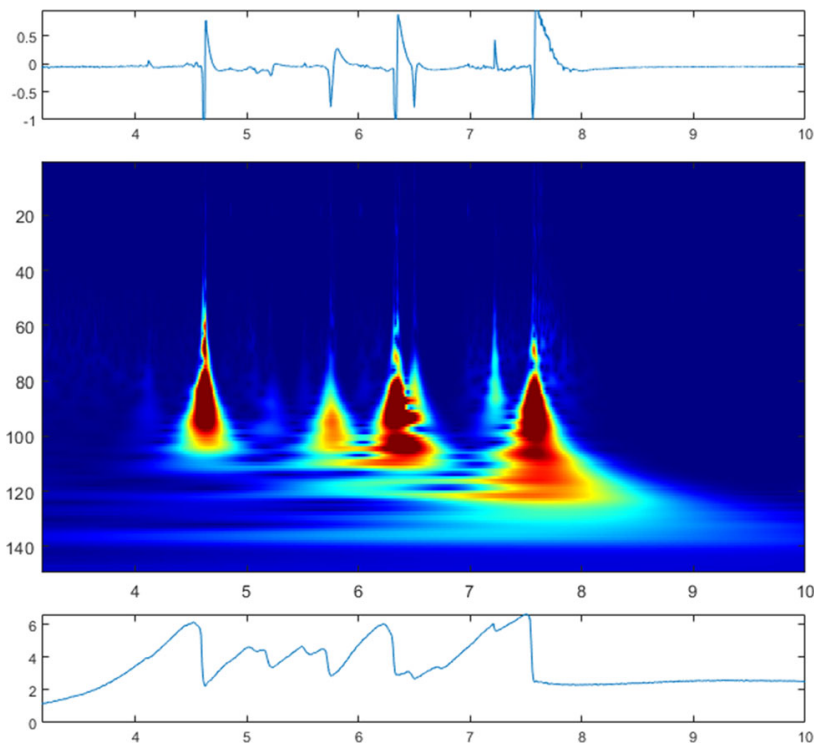

Fig. 2 The comparison of the audio (top) and the force (bottom) signal with the CWT-based feedback shows that events triggered by tissue-layer crossings can be clearly observed in the visual feedback

constructive for young surgeons with limited experience in performing laparoscopic access and obese patients, where the detection of tissue-layer crossings is challenging.

\section{Conclusion}

In this work, we explored the suitability of a visual and an auditory feedback variant for enhancing the surgeons' perception of tissuelayer crossings during laparoscopic access using the Veress needle. Different needle types were tested at various insertion velocities to evaluate if visual feedback based on Continuous Wavelet Transformation can accurately display tissue-layer crossings. Additionally, an add-on for auditory feedback has been implemented and tested by domain experts.

The results confirm that both feedback variants are accurate and enhance the surgeons' perception of tissue-layer crossings while being independent of the Veress needle's type and insertion velocities. The findings indicate that Surgical Audio Guidance could be an efficient means to improve precision and safety during laparoscopic access and motivate further research and development on the feedback variants presented in this paper.

\section{References}

[1] Alkatout I (2017) Complications of Laparoscopy in Connection with Entry Techniques. Journal of Gynecologic Surgery 33, 3 (2017), 81-91. https://doi.org/10.1089/gyn.2016.0111

[2] Schaufler A, Sühn T, Esmaeili N, Boese A, Wex C, Croner R, Friebe M, Illanes A. (2019) Automatic differentiation between Veress needle events in laparoscopic access using proximally attached audio signal characterization. Current Directions in Biomedical Engineering 5, 1 (2019), 369-371. https://doi.org/10.1515/cdbme-2019-0093 


\section{Non-rigid cutaneous tissue deformation estimation with iterative RANSAC and TPS from digital images over semi-ambiguous artificial markings}

\author{
C. $\mathrm{Shi}^{1}$, D. P. DeMeo ${ }^{2,3}$, E. L. Larson ${ }^{2}$, J. M. Galeotti ${ }^{1}$, \\ B. T. Carroll ${ }^{2,4}$ \\ ${ }^{1}$ Carnegie Mellon University, Robotics Institute, Pittsburgh, United \\ States ${ }^{2}$ University Hospitals, Department of Dermatology, Cleveland, \\ United States ${ }^{3}$ Case Western Reserve, School of Medicine, \\ Cleveland, United States ${ }^{4}$ Case Western Reserve, Department \\ of Dermatology, Cleveland, United States
}

Keywords dermatology, non-rigid deformation, RANSAC, thin plate spline

\section{Purpose}

Mohs micrographic surgery (MMS) is a dermatologic procedure that involves the removal of skin cancer while sparing as much healthy tissue as possible. MMS delivers decreasing cure rates with increasing sizes of cutaneous carcinoma. Postoperative complications increase with the increasing size of the reconstruction. Clinical errors may be introduced by biomechanical forces that deform tissue during routine manipulation during both MMS histologic processing and surgical flap reconstruction.

This interdisciplinary work seeks to ultimately reduce cancer recurrence and surgical complications by improving intra-operative surgical techniques that are used to remove and intra-operatively pathologically examine skin cancer. To that end, we seek to develop more accurate models of 3D tissue deformation during current clinical practice. To gather data on non-rigid tissue deformation, strain, and forces in real-world intra-operative surgery, we need new tools to study the 3D deformation of excised skin-cancer tissue, from its original in situ 3D shape to the final 3D shape when pressed against a surgical slide for intra-operative pathology. This current work is laying a foundation for those studies.

We have developed novel micro-painted fiducial landmarks that adhere to the fatty skin tissue. The focus of this paper is our custom computer-vision methodology for detecting, localizing, and matching those fiducial dots in tissue samples before-versus-after flattening on a microscope slide. The appearance of these landmarks makes it challenging to precisely define a center point for each dot, and the complex nonrigid tissue deformation, when pressed against a slide, makes it challenging to automatically match landmarks and compute the tissue-motion field.

\section{Methods}

Multichromatic acrylic microdots were painted onto porcine skin and tracked before and after hypodermal tissue bending (10 sample images) and epidermal flap reconstruction simulations (6 sample images). The painting process results in irregularly shaped dots of adhered acrylic. Two-dimensional microdot "center" coordinates were estimated by the blob detection algorithm from digital images and compared to a consensus of two expert-raters using two-tailed Welch"s t-tests. Color tagging, mutual information and Euclidean distance regulation were then used to measure the similarity between microdot pairs. For each microdot in either the before-surgery image or after-surgery image, we picked the microdot with the highest similarity with it in the other image as the best corresponded matching. When the bidirectional matching is mutually verified, then together with RANSAC, we established the correspondences between microdots from before and after images. The matching microdot pairs with high correlation values were used as key-point inputs to a thin plate spline method, warping the before image to register with the after image. Iteration over RANSAC matching and thin plate spline led to the final correspondence map for tissue deformation.

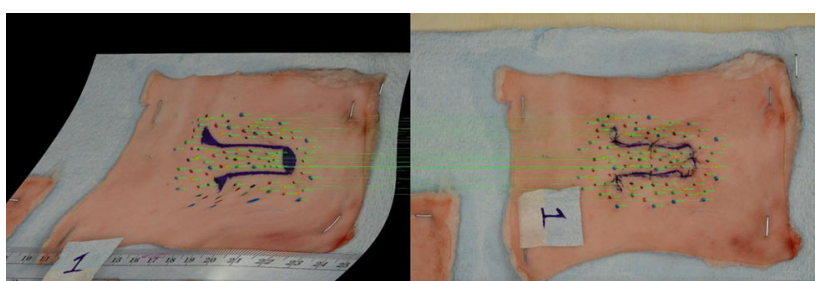

Fig. 1 Visualization of microdots matching between iterativelywarped before image and after image with location annotations (epidermis)

\section{Results}

The blob detection algorithm detected $83 \%$ of microdots overall Detection of microdots on epidermal flaps was higher before reconstruction than after, though not significantly ( $91 \%$ vs. $84 \%, p=0.16$ ), figure 1. There was no difference in detection of microdots on hypodermal tissue before and after bending ( $80 \%$ vs. $80 \%, p=0.87)$. Detection of microdots was higher on the epidermis than hypodermis ( $88 \%$ vs. $80 \%, p=0.01$ ). The correlation algorithm detected microdot coordinates within an average error of 11 pixels overall (corresponding to $201 \mu \mathrm{m}$, smaller is better). There was no difference in accuracy on epidermal flaps after reconstruction (5 vs. 7 px, $p=0.25$, corresponding to 170 vs. $209 \mu \mathrm{m}$ ), nor on hypodermal tissue after bending (13 vs. $16 \mathrm{px}, p=0.12$, corresponding to 189 vs. $231 \mu \mathrm{m})$. Accuracy was better on the epidermis than on the hypodermis (6 vs. 15 px, $p$.

Conclusion

Our custom microdots detection and matching algorithm based on iterative RANSAC and thin plate spline warping is an important step towards practically measuring biomechanical forces in the clinic using only digital images of irregular microdot fiducials. This largely decreases the labor work of manual annotations. It has been optimized for specificity over sensitivity, compared to expert-raters" annotation, and the algorithm shows sufficient pixel and micron accuracy to estimate deformation in exchange for a modest loss of resolution. Improving these techniques and introducing additional data such as three-dimensional point clouds, which will expand the applications of optical mapping in clinical research.

\section{References}

[1] Hruza GJ (1994) Mohs micrographic surgery local recurrences. J Dermatol Surg Oncol 20:573-577

\section{Ergonomic design and validation of an intraoperative system to measure plantar pressure distribution in supine position}

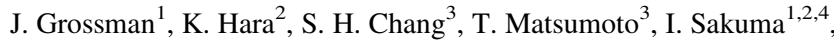
E. Kobayashi ${ }^{4}$

${ }^{1}$ University of Tokyo, Bioengineering, Bunkyo City, Japan

${ }^{2}$ University of Tokyo, Medical Device Development and Regulation Research Center, Tokyo, Japan ${ }^{3}$ University of Tokyo, Orthopaedic Surgery, Tokyo, Japan ${ }^{4}$ University of Tokyo, Precision Engineering, Tokyo, Japan

Keywords Intraoperative, Plantar pressure distribution, Hallux valgus, Transfer metatarsalgia

\section{Purpose}

Foot deformities such as hallux valgus are common foot deformities that may require surgery. However, during these surgeries many 
operative decisions are made based on inexact parameters. Consequently, surgical complications such as transfer metatarsalgia often arise [1]. Obtaining a proper plantar pressure distribution (PPD) is an important factor in the surgical success. However, it is difficult to measure intraoperatively since it is normally obtained in standing posture, while the surgery is performed in the supine position. A device which can measure this parameter in supine could improve the clinical result of surgical treatment by guiding surgeons to reliably restore a healthy standing plantar pressure distribution to the patient.

In previous research, an intraoperative plantar pressure measurement (IPPM) device was proposed, which can reproduce the PPD of standing posture intraoperatively [2]. It was found that in standing posture, the ground reaction force vector originates at the foot center of pressure and passes through the femoral head center. The IPPM device uses a force plate to align these two parameters, and a pressure sensor to measure PPD. The IPPM device demonstrated high reproducibility in experiments with healthy subjects, and now its usefulness must be clinically validated [2].

However, the IPPM device has two remaining issues that prevent it from being validated through clinical testing. One problem is its heavy hardware-it weighs $2.5 \mathrm{~kg}$. The other is that there is no convenient way for the user to hold and maneuver the device. These issues result in the device being difficult to operate, rendering it impractical for a clinical setting. Our research aims to redesign the IPPM device to be more ergonomic by solving the above two problems while maintaining its position measurement accuracy.

Methods

A. Reducing Hardware Weight

The original IPPM device"s force plate weighed $1.4 \mathrm{~kg}$. This force plate contained four force sensors, however, the parameters measured by the device can be measured by a single 6-axis force sensor. Hence, a new force plate was designed with only one sensor in the center of the plate, reducing the total weight by allowing the size of the force plate to be reduced. The new force sensor was chosen by its specifications for this application (FFS080YA501U6 Leptrino Co. Ltd.). The maximum force and moment limits of this sensor were determined from experiments conducted with the original device, where the maximum applied force during operation was around $100 \mathrm{~N}$.

The original force plate was made from aluminum which is a lightweight, strong, and inexpensive material. However, when optimizing strength and weight, composite materials are superior options. Carbon fiber was chosen due to its properties of being lightweight and high strength.

B. Improving Operability

To use the original device, the operator is forced to put their hands in a particular orientation to avoid contacting a measurement component and introducing error. As a result, operating the device can be strenuous. To improve maneuverability, a carbon fiber ring-shaped handle was attached to the back of the force plate.

C. Position Measurement Accuracy

To evaluate the position measurement accuracy of the new device, an optical tracking sensor (Polaris Spectra, NDI Co. Ltd.) was used. A tracking marker was pressed onto the device at different points, and the position of the marker measured by the sensor was compared to the position measured by the device $(\mathrm{N}=5)$. The required system accuracy for this test is less than $5 \mathrm{~mm}$ of position error.
Table 1 Comparison of the original and new device weight

\begin{tabular}{lll}
\hline Component weight $(\mathrm{g})$ & Old device & New device \\
\hline Pressure sensor & 700 & 500 \\
Top plate & 1400 & 400 \\
Force sensor & & 200 \\
Bottom plate & & 250 \\
Interfacing plate & 400 & N/A \\
Ring handle & N/A & 200 \\
Handle supports & N/A & 200 \\
Total & 2500 & 1750 \\
\hline
\end{tabular}

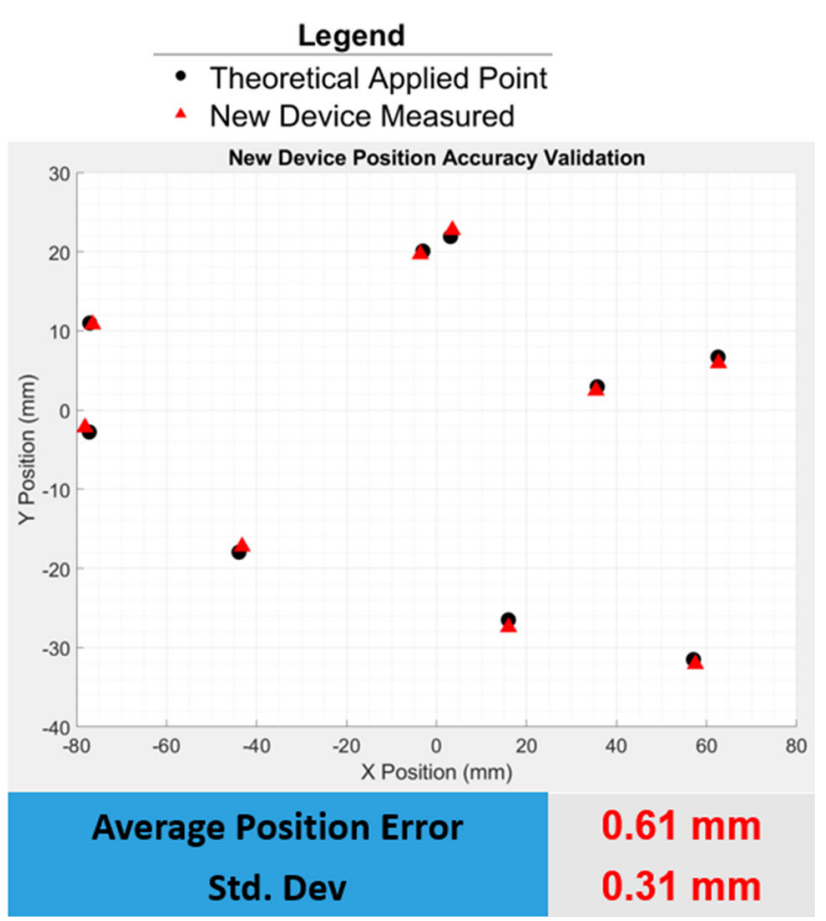

Fig. 1 Device Position Error Using Optical Tracking Sensor

\section{Results}

A comparison of the original and new device weight is shown in Table 1 . The original device weighs $2500 \mathrm{~g}$, and the new device weighs $1750 \mathrm{~g}$.

The new device design was qualitatively evaluated by two surgeons that had experience operating the original device. Both noted that the decreased weight along with the added handle provided a significantly more comfortable experience. Each surgeon concluded that the ergonomic design is appropriate for future clinical testing.

The results of the position measurement accuracy test are shown in Figure 1. The measured position error between the optical tracking sensor and the device mean was $0.61 \mathrm{~mm}$ (SD $0.31 \mathrm{~mm}$ ), which is below the system accuracy threshold of $5 \mathrm{~mm}$ of position error. There was no noticeable difference in measurement error for different positions on the device.

\section{Conclusion}

In this research, the IPPM device was redesigned to decrease its weight and improve its usability. Despite the inclusion of a $400 \mathrm{~g}$ 
ergonomic handle, the device weight was decreased by $30 \%$ without compromising its position measurement accuracy. Two surgeons confirmed the increase in usability from the new design and stated that the device design is suitable for clinical validation studies. In the future, clinical testing will be done to determine the system"s ability to reproduce plantar pressure distribution in a clinical environment.

\section{References}

[1] Coughlin MJ, Shurnas PS (2003) Hallux rigidus: demographics, etiology, and radiographic assessment. Foot Ankle Int 24(10):731-743

[2] Hosoi I, Kobayashi E, Chang S, Matsumoto T, An Q, Anzai E, Ohta Y, Sakuma I (2018) "Development of intraoperative plantar pressure measuring system considering weight bearing axis", International Journal of Computer Assisted Radiology and Surgery, vol. 14, no. 2, pp. 385-395.

\section{$\overline{\text { Smartphone-based augmented reality system for needle }}$ insertion guidance in sacral nerve stimulation}

R. Moreta-Martinez ${ }^{1,2}$, M. García-Sevilla ${ }^{1,2}$, D. García-Mato ${ }^{1,2}$, A. Pose-Díez-de-la-Lastra ${ }^{1,2}$, I. Rubio-Pérez ${ }^{3}$, J. Pascau ${ }^{1,2}$

${ }^{1}$ Universidad Carlos III de Madrid, Departamento de Bioingeniería e Ingeniería Aeroespacial, Madrid, Spain ${ }^{2}$ Instituto de Investigación Sanitaria Gregorio Marañón, Madrid, Spain ${ }^{3}$ Hospital Universitario La Paz, Servicio de Cirugía General, Madrid, Spain

Keywords Sacral nerve stimulation, Augmented reality, Needle guidance, Smartphone

\section{Purpose}

Sacral nerve stimulation (SNS) is a procedure where an electrode is implanted through the sacral foramina to stimulate the nerve modulating colonic and urinary functions. This practice has been implemented efficiently to treat several pathologies such as fecal incontinence, urinary retention, and constipation.

Currently, X-ray fluoroscopy is used during electrode placement to estimate the location of the needle with respect to the sacral foramina (usually S3 or S4). However, this needle insertion is very challenging to surgeons, and several X-ray projections are required to interpret the needle"s position correctly. Furthermore, the need for multiple punctures causes an increase in surgical time and patients" pain.

Navigation systems based on optical tracking combined with intraoperative CT have been previously used to guide needle insertion in SNS surgeries, reducing procedural time and improving surgical outcomes. Nevertheless, these systems require an intraoperative CT and imply additional radiation exposure during the intervention to both patients and professionals, restricting their clinical practice integration. Additionally, the navigation information is displayed on external screens, requiring the surgeon to divert his attention from the patient. In this context, augmented reality (AR) technology could overcome these limitations, providing the surgeon with real-time navigation information directly overlaid in the surgical field and avoiding external radiation during surgery [1].

In this work, we propose a smartphone-based AR application to guide electrode placement in SNS surgeries. This navigation system uses a 3D-printed reference marker placed on the patient to display virtual guidance elements directly on the affected area, facilitating needle insertion with a predefined trajectory. The proposed system has been evaluated on an anthropomorphic phantom.

Methods

A patient-based phantom was manufactured to simulate the affected area for an SNS treatment. The phantom included the sacrum bone,
3D-printed in polylactic acid, covered with silicon (Dragon Skin 10 Slow), imitating the patient's soft tissue. The location of the sacrum foramen was obtained by segmenting both materials from a CT of the phantom using 3D Slicer software.

We developed a smartphone AR application on Unity platform. This app uses Vuforia development kit to detect and track the position of a 3D-printed cubic reference marker $(30 \times 30 \times 30 \mathrm{~mm})$ with unique black-and-white patterns on each face. Once the marker is detected in the smartphone camera field of view, the virtual models are displayed overlaid on the real-world image. These virtual models will indicate the insertion point on the surface of the phantom and the optimal trajectory to reach the target sacral foramen (Figure 1).

The marker was fixed on top of the phantom in the superior area of the gluteus. We obtained the position of the marker with respect to the phantom by a two-step procedure. Firstly, we acquired a 3D photograph (including geometric and textural information) of the phantom"s surface with the cubic reference marker already in place. With this information, we applied a surface-to-surface registration algorithm to align the acquired 3D textured with the phantom model obtained from the CT scan. Secondly, we identified seven landmarks from the marker patterns on the 3D textured images. These landmarks were used to compute the position of the marker with respect to the phantom after a fiducial-based registration. Finally, the virtual models" position was calculated and uploaded to the AR application.

We evaluated our solution during needle insertion on several simulated SNS interventions on the manufactured phantom. After detecting the AR marker with the smartphone"s camera, the user held the smartphone with one hand and inserted the needle with the other one. The trajectory and the target were displayed as virtual elements on the AR-display. Once the needle was oriented as indicated in the AR system, the user inserted the needle. A total of three inexperienced users performed this procedure ten times on two sacrum foramina of different sizes (S3 and S4). We measured the insertion time and the number of punctures required to place the needle in the target foramen for each repetition.

Results

Table 1 shows the insertion time and the number of punctures for each user and foramen. The average insertion time was $30.5 \pm 13.9 \mathrm{~s}$, slightly lower than the figures reported with alternative SNS guidance methods ( $35.4 \pm 14.6 \mathrm{~s}$ ) [2]. The results obtained with the proposed system showed that users performed a maximum of two punctures to reach the target for both foramina with an average of $1.13 \pm 0.34$,

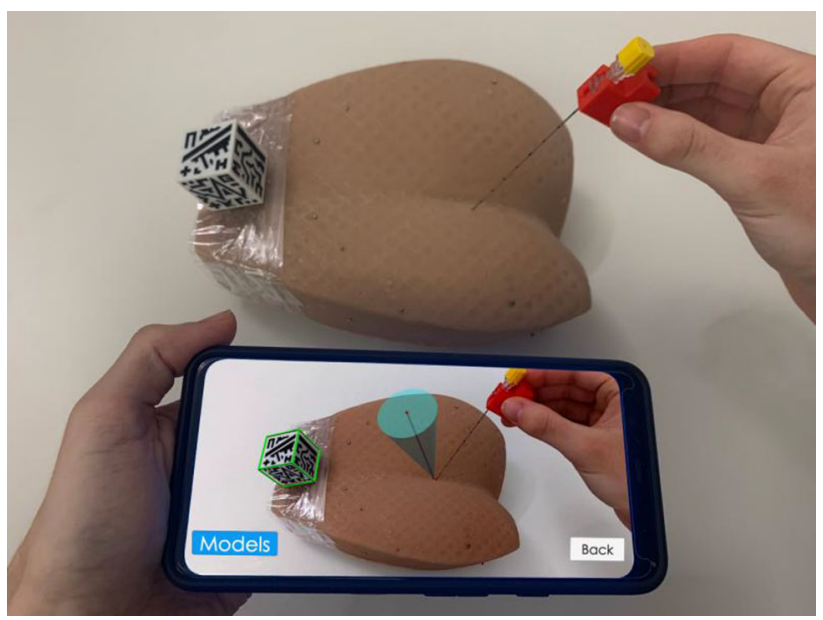

Fig. 1 User during needle insertion simulation on the manufactured phantom using the smartphone-based augmented reality application for needle guidance 
Table 1 Number of punctures and insertion time for each foramen and user during simulation with the proposed augmented reality system

\begin{tabular}{|c|c|c|c|c|c|c|c|c|}
\hline & \multicolumn{4}{|c|}{ Foramen S3 } & \multicolumn{4}{|c|}{ Foramen S4 } \\
\hline & User 1 & User 2 & User 3 & Avg. users & User 1 & User 2 & User 3 & Avg. users \\
\hline \multicolumn{9}{|c|}{ Puncture time (s) } \\
\hline Mean & 27.1 & 34.3 & 29.8 & 30.4 & 25 & 36.3 & 30.7 & 30.6 \\
\hline Std & 10.1 & 16.6 & 16 & 14.4 & 5.9 & 18.7 & 11.9 & 13.6 \\
\hline Min & 18 & 18 & 15 & 17 & 20 & 17 & 23 & 20 \\
\hline Max & 45 & 71 & 70 & 62 & 40 & 69 & 62 & 57 \\
\hline \multicolumn{9}{|c|}{ Number of punctures } \\
\hline Mean & 1 & 1.2 & 1.1 & 1.1 & 1.2 & 1.2 & 1.1 & 1.2 \\
\hline Std & 0 & 0.4 & 0.3 & 0.3 & 0.4 & 0.4 & 0.3 & 0.4 \\
\hline
\end{tabular}

significantly reducing the average number of insertions reported with traditional methods $(9.6 \pm 7.7)[2]$.

\section{Conclusion}

This work proposes a novel smartphone-based AR navigation system to improve electrode placement in sacral neuromodulation procedures. The results obtained on the patient-based phantom show that our system could reduce the number of punctures and the insertion time compared with the traditional methods. The smartphone application is intuitive, facilitating needle insertion without requiring tool tracking. Further studies should be performed to ensure feasibility during surgical interventions. To our knowledge, this is the first work proposing an AR solution to guide needle insertion in SNS procedures.

\section{Acknowledgements}

Supported by projects PI18/01625 (Ministerio de Ciencia, Innovación y Universidades, Instituto de Salud Carlos III and European Regional Development Fund Una manera de hacer Europa) and IND2018/TIC9753 (Comunidad de Madrid).

\section{References}

[1] García-Mato D, Moreta-Martinez R, García-Sevilla M, Ochandiano S, García-Leal R, Pérez-Mañanes R, Calvo-Haro JA, Salmerón JI, Pascau J (2020) Augmented reality visualization for craniosynostosis surgery. Comput Methods Biomech Biomed Eng Imaging Vis 1-8. https://doi.org/10.1080/ 21681163.2020.1834876

[2] Zhang J, Zhang P, Wu L, Su J, Shen J, Fan H, Zhang X (2018) Application of an individualized and reassemblable 3D printing navigation template for accurate puncture during sacral neuromodulation. Neurourol Urodyn 37:2776-2781. https://doi.org/ 10.1002/nau.23769

\section{Three-dimensional surgical plan printing for assisting liver surgery}

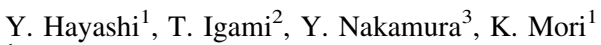

${ }^{1}$ Nagoya University, Graduate School of Informatics, Nagoya, Japan

${ }^{2}$ Nagoya University, Graduate School of Medicine, Nagoya, Japan

${ }^{3}$ National Institute of Technology, Tomakomai College, Department of Engineering for Innovation, Tomakomai, Japan

Keywords 3D print, Liver, Surgical plan, Image-guided surgery

\section{Purpose}

Computer assisted surgery (CAS) system, which includes surgical planning system and surgical navigation system, has been developed to assist understanding of patient specific anatomical structures in the liver. These systems enable surgeon to perform preoperative surgical planning and intraoperative surgical navigation of the liver resections while observing the $3 \mathrm{D}$ reconstructed patient specific anatomical structures from CT volumes. In resent year, since three dimensional (3D) print technologies have been rapidly developed, a 3D printed organ model is widely spread in medical field. The 3D printed organ model makes easy to recognize 3D positional relationships among anatomical structures. Therefore, in addition to CAS system, a 3D printed liver model is also used for assisting liver surgery [1,2]. In the 3D printed liver model, patient specific anatomical structures in the liver, such as the portal vein and the hepatic vein, are usually fabricated. Surgeon can comprehend positional relationships among these anatomical structures and tumors in the liver by observing the 3D printed liver model preoperatively and intraoperatively. Surgeon can also plan liver resections by considering these positional relationships. If the surgical plan such as liver partition line reproduced in the $3 \mathrm{D}$ printed liver model in addition to the anatomical structures, the $3 \mathrm{D}$ printed model will be more useful for assisting liver resection surgery. In this paper, we describe the three-dimensional surgical plan printing for assisting liver resection surgery.

\section{Methods}

The proposed method fabricates two 3D printed liver models separated by liver partition line planned preoperatively as surgical plan printing. The proposed method consists of image processing part and fabrication part. In the image processing part, the anatomical structures are extracted from CT volume and the liver partition line is planned. In the fabrication part, the 3D liver model is fabricated based on the result of the image processing part using a $3 \mathrm{D}$ printer. We extract anatomical structures semi-automatically from portal venous phase contrast-enhanced CT volumes. The liver, the portal vein, the hepatic vein, and the tumor regions are extracted using region growing method and morphological operations such as opening and closing. Surgeon performs surgical planning by considering the locations of the tumor and its surrounding anatomical structures on CT volumes. Voronoi tessellation is performed to obtain liver partition line based on the portal vein information. Surgeon checks and corrects the segmentation results and liver partition line manually if necessary. The Blood vessel regions are dilated by morphological operation to reproduce the thin blood vessels. The blood vessel and tumor regions are subtracted from the liver region. The liver partition line also subtracted from the liver regions to divide the liver model. We convert the obtained binary images to polygon data using marching cubes algorithm. The two 3D printed liver models is fabricated the polygonal model using a 3D printer (Agilista 3100, 


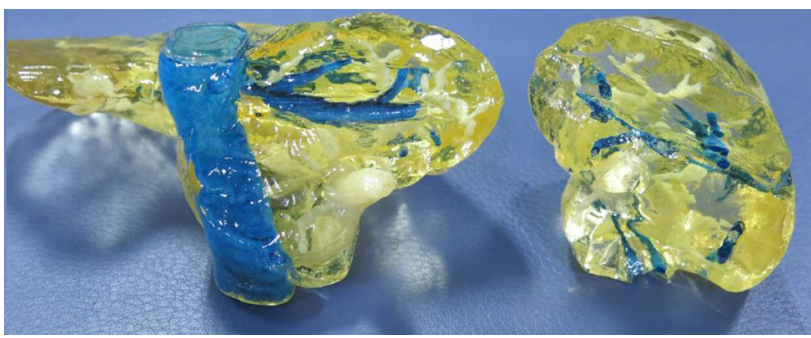

Fig. 1 Example of fabricated 3D printed liver model. 3D printed liver model is divided two models by planned liver partition line

Keyence, Osaka, Japan). After fabrication, support material covered with the 3D printed liver models is removed. The surface of the 3D liver models is polished by abrasive sponge and coated by urethane resin to smooth the surface. The portal vein and tumor is colored white by filling support material. The hepatic vein regions are colored by blue dye after removing the support material.

\section{Results}

We created the 3D liver models using the proposed method. Figure 1 shows an example of the fabricated 3D printed liver model. This 3D liver model is divided into two models by the planned liver partition line. Since the liver regions are fabricated translucent acrylic resin, the anatomical structures in the liver can be observed. The portal vein and the tumor are showed white color, and the hepatic vein is showed blue color in the model. The positional relationships between the portal vein, the hepatic vein, and the tumor inside the liver are confirmed using the fabricated 3D liver model. Furthermore, the anatomical structures around the planned liver partition line are also confirmed by using the divided 3D liver model. This will be helpful to surgeon for understanding the complex anatomical structures around partition line during the liver resection. Therefore, the 3D printed liver model with surgical plan is useful for assisting liver surgery. Conclusion

In this paper, we described a three dimensional surgical plan printing for assisting liver resection surgery. The proposed method created two 3D printed liver models divided by the preoperative liver partition plan. The experimental results showed that the anatomical structures along the planned partition line are confirmed using the fabricated 3D printed liver models. Future works includes application to additional cases and development of an automated segmentation method of anatomical structures from $\mathrm{CT}$ volumes.

\section{References}

[1] Igami $T$, Nakamura $Y$, Hirose $T$, Ebata $T$, Yokoyama $Y$, Sugawara G, Mizuno T, Mori K, Nagino M (2014) Application of a three-dimensional print of a liver in hepatectomy for small tumors invisible by intraoperative ultrasonography: preliminary experience. World J Surg 38(12): 3163-3166

[2] Igami T, Nakamura Y, Oda M, Tanaka H, Nojiri M, Ebata T, Yokoyama Y, Sugawara G, Mizuno T, Yamaguchi J, Mori K, Nagino M (2018) Application of three-dimensional print in minor hepatectomy following liver partition between anterior and posterior sectors. ANZ J Surg 88(9): 882-885
Surgical procedure simulation dataset for surgical robot development: an example of canine spay surgery

P. Suteecharuwat ${ }^{1}$, N. Kubuki ${ }^{1,2}$, Y. Ran ${ }^{2}$, K. Nagase ${ }^{1}$, Y. Hayakawa ${ }^{3}$ ${ }^{1}$ Kitami Institute of Technology, Master's Course of Computer Science, Graduate School, Kitami, Hokkaido, Japan ${ }^{2}$ Kitami Institute of Technology, Dept. of Computer Science, Kitami, Hokkaido, Japan ${ }^{3}$ Kitami Institute of Technology, Dept. of Engineering on Intelligent Machines \& Biomechanics, Kitami, Hokkaido, Japan

Keywords Surgical simulation dataset, simulation dataset, image recognition, annotation

\section{Purpose}

Development of autonomous surgical robotic machines for standard surgical procedures is under requisition rather than master-slave machines. Surgery with standardized procedures is technically in high demand as an autonomous surgical robot in local cities where the number of surgeons is decreasing. However, it is necessary to obtain the patient consent when collecting simulation data, and there are crucial hurdles in such data collection. Therefore, we created a dataset that using a simulation model of contraceptive surgery, which is a routine surgery for small animals, dogs. Ovariohysterectomy is the common sterilization procedure for small animals, namely cats and dogs, etc. In the study, we tried the simulation of the canine (dog) spay surgery, ovariohysterectomy, and made the data set of the surgery.

\section{Methods}

The automatic image recognition of tools (surgical instruments) and surgical operation (work-flow) phases were carried out using image recognition technology. Using a simulation model of the canine spay surgery, 42 video movies $(1080 \times 720$ pixels, $24 \mathrm{fps})$, in the range of $15 \mathrm{~min}$ to $25 \mathrm{~min}$ in length, were recorded during every surgical procedure (Fig. 1). The annotation of surgical tools was carried out in the first 15 video movies (down-sampling to $1 \mathrm{fps}$ ) by means of the bounding-box annotation.

\section{Results}

The annotation of surgical tools was carried out in the first 15 video movies (down-sampling to $1 \mathrm{fps}$ ) by means of the bounding-box annotation. Two or more tools are annotated on more than 80 percent in 16,327 frames in total. This method is fitting for the precise object detection having the individual locational data. Since surgical tools are often held by hand and it is not easy to annotate the entire tool, only a part of the tool was annotated. The scissors, needle holder, and forceps are very similar when viewed from the side, and there is a problem that they cannot be discriminated by the human eye. Therefore, when annotating the needle holder that sandwiches the

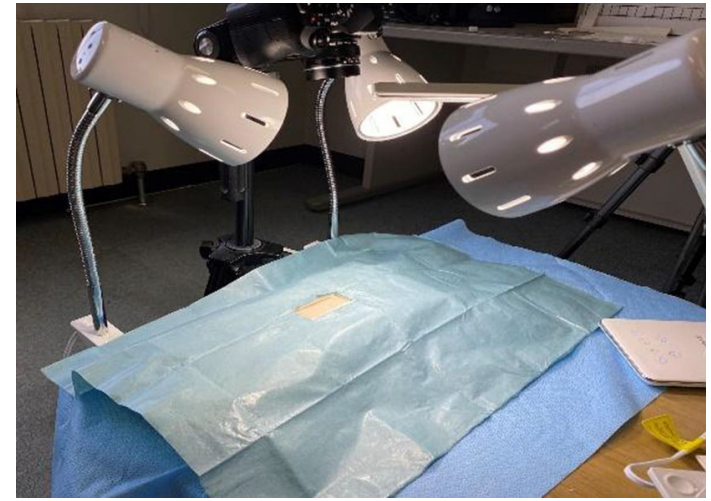

Fig. 1 Forty-second videos was taken for surgical practice and has a resolution of $1920 \times 1080$.Intraoperative lighting setting: bright 


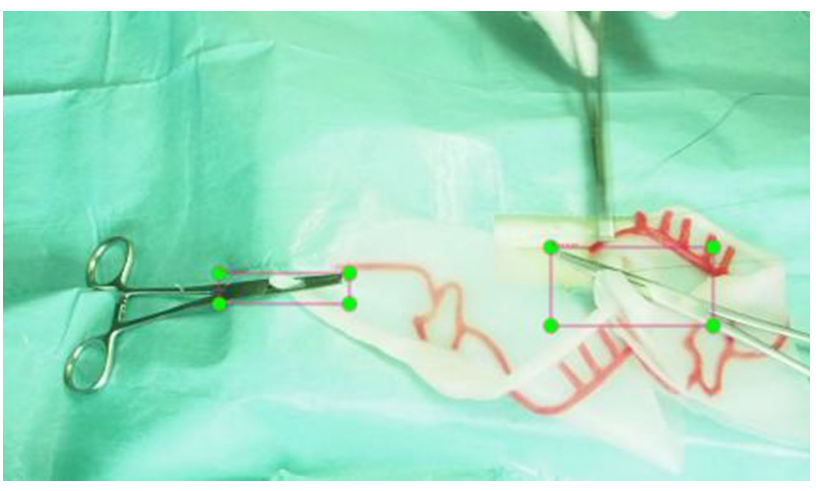

Fig. 2 An example of tool annotation. Needle holder, holding the suture needle

suture needle, we improved it so that the needle holder can be distinguished by attaching a bounding box together with the suture needle. By annotating in this way, it is considered that the recognition accuracy when the image of the needle holder is blurred can be improved (Fig. 2). Suture needles were also used, but, for these three reasons, that the size of the suture needle is small, it is rarely seen depending on the angle, and it is often pinched by a needle holder, we didn't annotate the bounding-box individually. The annotation of surgical operation (work-flow) phases was carried out in all 42 video movies. Six consecutive phases in the canine spay surgery were recognized. Since selected tools are used in specific phases, correlations between tools and phases are helpful for the automatic phase recognition.

\section{Conclusion}

We made the data set for tool recognition and phase recognition while the canine spay surgery is carried out using a simulation model. The annotation data-set of surgical instruments (tools) and surgical operation (work-flow) phases were obtained using the simulation of the canine spay surgery, ovariohysterectomy. The next step is the designing of a neural network to recognize these tools and surgical phases. We had an opportunity to collaborate with veterinary surgeons in a veterinary medicine school. We also plan the similar simulation in oral and maxillofacial surgical procedures.

\section{References}

[1] Jin A., Yeung S, Jopling J, Krause J, Azagury D, Milstein A, Fei-Fei L (2018) Tool detection and operative skill assessment in surgical videos using region-based convolutional neural networks. 2018 IEEE Winter Conference on Applications of Computer Vision (WACV). IEEE

\section{Real-time bone fragment navigation for open cranial vault remodeling}

D. García-Mato ${ }^{1,2}$, M. García-Sevilla ${ }^{1,2}, \mathrm{~S}$. Ochandiano ${ }^{2,3}$, J. Pascau ${ }^{1,2}$

${ }^{1}$ Universidad Carlos III de Madrid, Departamento de Bioingeniería e Ingeniería Aeroespacial, Madrid, Spain ${ }^{2}$ Instituto de Investigación Sanitaria Gregorio Marañón, Madrid, Spain ${ }^{3}$ Hospital General Universitario Gregorio Marañón, Servicio de Cirugía Oral y Maxilofacial, Madrid, Spain

Keywords computer-assisted surgery, craniosynostosis, 3D printing, craniofacial surgery

\section{Purpose}

Craniosynostosis is a congenital defect characterized by the premature fusion of one or more cranial sutures. This medical condition usually leads to dysmorphic cranial vault and may cause functional problems. Surgical correction is the preferred treatment to excise the fused sutures and to normalize the cranial shape of the patient.

Open cranial vault remodeling is the standard surgical technique for the correction of craniosynostosis. This approach consists of three steps: osteotomy and removal of the affected bone tissue, reshaping the bone fragments into the most appropriate configuration, and placement and fixation of the reshaped bone fragments to achieve the desired cranial shape [1]. Nowadays, surgical management of craniosynostosis is still highly dependent on the subjective judgment of the surgeons and, therefore, there is high variability in the surgical outcomes. Inaccuracies in osteotomy and remodeling can compromise symmetry, harmony, and balance between the face and the cranial vault and, therefore, the aesthetic outcome.

In a previous work [2], we presented a novel workflow for intraoperative navigation during craniosynostosis surgery. This system requires a tracked pointer tool to estimate the positions of bone fragments by recording points along their surface. Although this methodology presents a high accuracy, it is time-consuming and does not enable real-time tracking of the bone fragments. Continued monitoring of individual fragments' position would facilitate intraoperative guidance and improve matching with the virtual surgical plan.

In this study, we present and evaluate a novel workflow for realtime tracking of bone fragments during open cranial vault remodeling combining patient-specific 3D printed templates with optical tracking. The proposed methodology was evaluated through surgical simulations in a $3 \mathrm{D}$ printed phantom.

\section{Methods}

A 3D printed phantom was designed and manufactured to replicate a realistic scenario for surgical simulation and performance evaluation. This phantom is based on data from a patient with metopic craniosynostosis previously treated in our center. Bone and soft tissue were simulated with polylactic acid and silicone materials, respectively.

Experienced craniofacial surgeons performed a virtual surgical plan to define the location of osteotomies to remove the affected bone tissue, the best approach to reshape the bone fragments, and the optimal position of the fragments in the patient to achieve the desired cranial shape. Two different interventional plans were defined to create two distinct scenarios for surgical simulation: a simple plan, with symmetric overcorrection and adjacent to anatomical landmarks; and a complex plan, with asymmetric overcorrection and distant to characteristic anatomical References.

We developed a surgical navigation system based on optical tracking and desktop 3D printing to guide surgeons during the intervention (see figure 1). First, a patient-specific template was designed and $3 \mathrm{D}$ printed according to the virtual surgical plan. This template enables the surgeons to reshape the bone fragments of the supraorbital region as defined during planning. In addition, this template incorporates spherical optical markers for position tracking. Then, the reshaped bone fragments can be attached to the template and their position can be computed in real-time by the optical tracking system. To our knowledge, this is the first approach to track the position of bone fragments using patient-specific 3D printed templates.

A software application was specifically developed to display the $3 \mathrm{D}$ position of the bone fragments during surgery with respect to the patient's anatomy, providing visual and acoustic feedback to the surgical team to ensure optimal placement and fixation according to the preoperative virtual plan. A 3D printed reference frame is attached to the patient to compensate for possible movements during surgical 


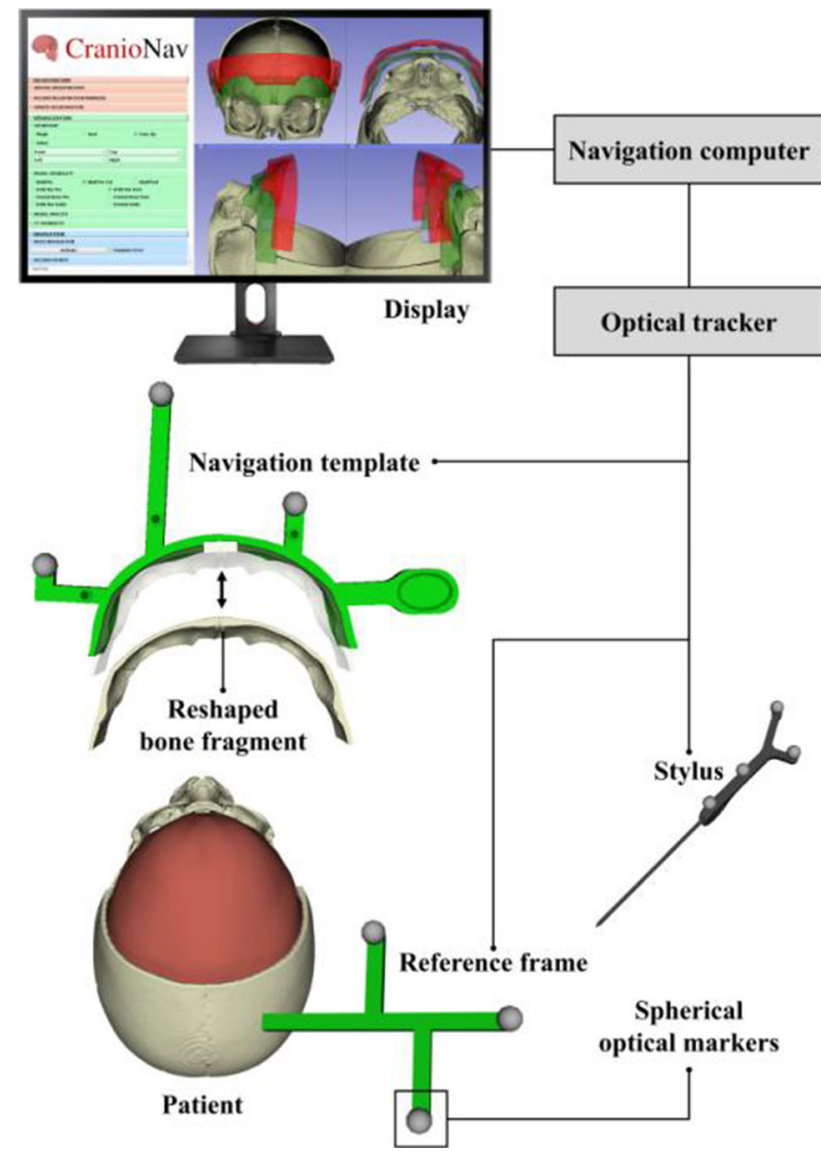

Fig. 1 Intraoperative navigation system for open cranial vault remodeling

intervention, and a stylus tool is used to record landmarks for patientto-image registration.

The accuracy of this navigation system was evaluated by simulating the surgical intervention using a $3 \mathrm{D}$ printed phantom. A total of 20 surgical simulations were performed in two scenarios with different complexity (i.e. simple and complex virtual plans). An experienced user performed 10 simulations using the navigation system, and 10 simulations using the standard freehand approach (without navigation). The final position of the supraorbital bone fragment was computed by recording the position of 5 pinholes using the optical tracker. This position was compared with the virtual plan, and translation and rotation errors were computed for each simulation.

\section{Results}

Average translation and rotation errors show increased performance when using the navigation system (see table 1). The navigated simulations showed a similar positioning accuracy in both simple and complex scenarios, presenting an average error below $1 \mathrm{~mm}$ in translation and 1 degree in rotation. However, the standard freehand approach showed a lower accuracy in the complex surgical scenarios, with maximum errors of $7.91 \mathrm{~mm}$ in translation and 5.21 degrees in rotation.

Conclusion

The proposed navigation workflow enables an accurate reshaping and positioning of the remodeled bone fragments to match the preoperative virtual plan. In contrast with previous approaches, this technique provides surgeons with real-time $3 \mathrm{D}$ visualization and metrics to accurately control the bone fragment position during cranial remodeling. Our framework outperforms the standard freehand approach for
Table 1 Average translation and rotation errors in the positioning of the remodeled supraorbital fragment for two different scenarios: simple and complex

\begin{tabular}{|c|c|c|c|c|c|c|c|}
\hline \multirow[t]{2}{*}{ Scenario } & \multirow[t]{2}{*}{ Mode } & \multicolumn{3}{|c|}{ Translation error $(\mathrm{mm})$} & \multicolumn{3}{|c|}{ Rotation error $\left(^{\circ}\right)$} \\
\hline & & Mean & Std. & Max. & Mean & Std. & Max. \\
\hline \multirow[t]{2}{*}{ Simple } & Freehand & 1.5 & 0.61 & 2.47 & 2.37 & 1.11 & 4.45 \\
\hline & Navigated & 0.52 & 0.33 & 1.16 & 0.68 & 0.52 & 1.74 \\
\hline \multirow[t]{2}{*}{ Complex } & Freehand & 2.85 & 2.36 & 7.91 & 2.04 & 1.48 & 5.21 \\
\hline & Navigated & 0.56 & 0.29 & 1.09 & 0.65 & 0.65 & 1.77 \\
\hline
\end{tabular}

remodeling in both simple and complex surgical scenarios, showing a higher accuracy and potential to improve surgical outcomes.

Our novel methodology based on intraoperative navigation and 3D printing can be integrated into the current surgical workflow to ensure an accurate translation of the preoperative surgical plan into the operating room. This solution could improve the reproducibility of surgical interventions and reduce inter-surgeon variability.

Acknowledgements

Supported by projects PI18/01625 (Ministerio de Ciencia, Innovación y Universidades, Instituto de Salud Carlos III and European Regional Development Fund "Una manera de hacer Europa") and IND2018/ TIC-9753 (Comunidad de Madrid).

\section{References}

[1] Cohen SR, Frank RC, Meltzer HS, Levy ML (2014) Craniosynostosis. Handb Craniomaxillofacial Surg 343-368. https://doi.org/10.1142/9789814295109_0013

[2] García-Mato D, Ochandiano S, García-Sevilla M, NavarroCuéllar C, Darriba-Allés JV, García-Leal R, Calvo-Haro JA, Pérez-Mañanes R, Salmerón JI, Pascau J (2019) Craniosynostosis surgery: workflow based on virtual surgical planning, intraoperative navigation and 3D printed patient-specific guides and templates. Sci Rep 9:17691. https://doi.org/10.1038/ s41598-019-54148-4

\section{Towards machine learning-based tissue differentiation using an ultrasonic aspirator}

N. Bockelmann ${ }^{1}$, D. Kesslau ${ }^{2}$, M. M. Bonsanto ${ }^{3}$, S. Buschschlüter ${ }^{2}$, F. Ernst $^{1}$

${ }^{1}$ Institute for Robotics and Cognitve Systems, University of Lübeck, Lübeck, Germany ${ }^{2}$ Söring GmbH, Quickborn, Germany ${ }^{3}$ Department of Neurosurgery, University Hospital Schleswig-Holstein, Lübeck, Germany

Keywords Tissue differentiation, Tactile sensor, Ultrasonic aspirator, Machine learning

\section{Purpose}

In addition to visual assessment for tumor differentiation, palpation of tissue by the surgeon during surgery is of great importance, for example during resection of intracranial lesions. This intraoperative differentiation of soft tissue can be a challenging task that usually requires years of expertise and intuition. For this reason, research is currently being conducted on tactile sensors as an assistive device for better tumor delineation. However, these sensors are typically intended for robotic applications which are not usable in every surgical 
case and are required to measure the force applied to the tissue. This leads to the development of new devices that must be integrated into the surgical workflow. On the other hand, ultrasonic aspirators are commonly used as hand-held instruments during tumor resection. Since ultrasonic aspirators and piezoelectric tactile sensors operate in a similar manner, the idea of using a commercially available ultrasonic aspirator as an intelligent intraoperative probe for tissue differentiation is motivated. This eliminates the need to change instruments during surgery and improves the workflow of the surgeons. The final aim is to predict the tissue properties by only using the electrical features of the ultrasonic aspirator, and by that compensating for the missing contact force which is not available in this surgical instrument. In the following, it is investigated as a first step in a simplified laboratory setting, whether machine learning methods can learn a relationship between the electrical features of an ultrasonic aspirator and the mechanical properties of different tissues.

\section{Methods}

Data is acquired using four synthetically created tissue models. Each tissue model represents a different tissue consistency and is characterized with a stiffness value that is derived from its chemical contents. This means that the higher the stiffness value, the higher the tissue model consistency. Data acquisition is done with a $\mathrm{CNC}$ machine that holds the ultrasonic aspirator directly over the tissue model, brings it into contact and moves up again while exerting as little force on the tissue model as possible. Furthermore, the settings of the instrument are set to a non-resection mode so that the tissue model remains intact. For each tissue model, seven or eight recordings are made, each having a length of 12-24 s at a recording frequency of $21 \mathrm{~Hz}$. This results in a total of 30 recordings with more than 11,000 data points, each of which containing the corresponding stiffness value and several electrical features of the ultrasonic aspirator.

Since tissue properties can take different continuous values, regression is performed on the different stiffness values using the electrical features as input. In order to perform a regression on this data, three empirically determined nonlinear methods are used. The first two methods are deep learning-based regression models: a Fully Connected Network (FCN) that takes the features as input to regress to the respected stiffness value and a 1D Residual Network (ResNet) which uses a sliding windows approach with a window size of four seconds to regress to the center point of the window. While the first method is only taking information of the current data into account for the regression, the latter method tries to leverage temporal information of the adjacent data to increase performance. The last method uses a Gaussian Process (GP) with an exponential kernel to determine the stiffness value. An advantage of using GPs for a regression is the incorporation of uncertainty into the predictions, which can be beneficial in later applications.

\section{Results}

A five-fold cross-validation is conducted over the 30 recordings with a total of more than 11,000 data points. The same splits are used across the different methods and normalized to have zero mean and unit variance. Data points without any contact to the tissue models are assumed to have a stiffness value of zero. To evaluate the performance of the methods, the metrics root-mean-square error (RMSE), mean absolute error (MAE) and $\mathrm{R}^{2}$ are obtained and averaged over the five folds. The summarized results of the regression can be found in Table 1. For all methods, a high $\mathrm{R}^{2}$ value of more than 0.9 can be obtained. Since the two deep learning-based methods show similar results it can be deduced that there is limited impact of temporal information to the predictions. The GP regressor model yields superior performance compared to the deep learning-methods. A qualitative result of the GP can be found in Fig. 1. Particularly noteworthy are the large uncertainties in the area of the jump discontinuities of the signal and the samples around. Those jump discontinuities are caused by bringing the instrument into contact with
Table 1 Results of different methods on five-fold cross-validation in mean \pm standard deviation

\begin{tabular}{llll}
\hline Methods & RMSE & MAE & $\mathrm{R}^{2}$ \\
\hline FCN & $0.894 \pm 0.619$ & $0.473 \pm 0.409$ & $0.906 \pm 0.106$ \\
ResNet & $0.823 \pm 0.870$ & $0.454 \pm 0.621$ & $0.903 \pm 0.188$ \\
GP & $0.561 \pm 0.539$ & $0.286 \pm 0.365$ & $0.940 \pm 0.092$ \\
\hline
\end{tabular}

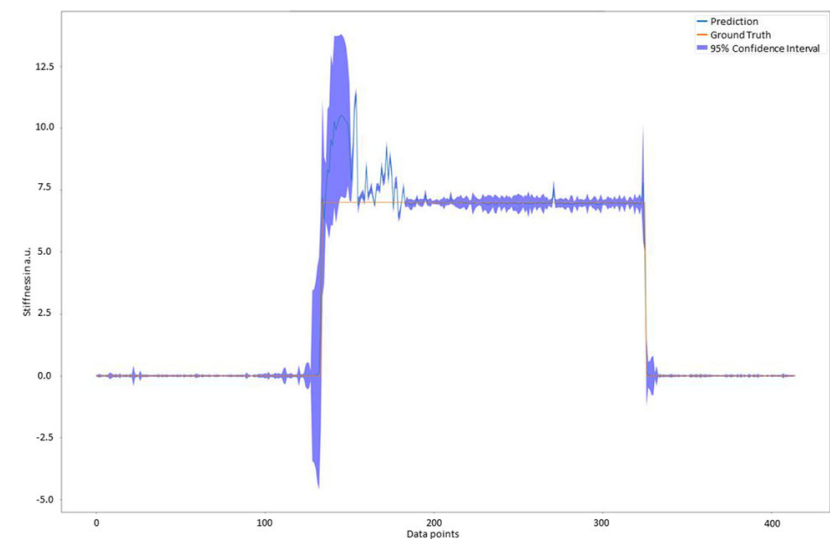

Fig. 1 Example of regression with GP on 400 test data points. In areas with large deviation of ground truth, high uncertainty occurs

the tissue model and removing it again, and pose a challenge in the methods investigated. However, the communication of the uncertainty of the model's prediction to the user allows to reject the prediction in a later clinical application, which can be particularly relevant in this safety-critical environment.

\section{Conclusion}

This work shows the possibility to learn a relationship between the electrical features of an ultrasonic aspirator and the mechanical properties of tissue models with a $\mathrm{R}^{2}$ metric of more than 0.9 . This indicates the feasibility to distinguish between different tissue types using the surgical instrument. Future work needs to investigate the performance on a larger variety of mechanical properties and the influence of contact force. Furthermore, a more in-depth analysis of the temporal influence to the regression is necessary, especially during initial and final contact with the tissue model. In addition, performance on data involving resection of tissue needs to be investigated to pave the way for later clinical application.

This work was funded by the German Federal Ministry for Economic Affairs and Energy (BMWi, project KI-Sigs, grant number: 01MK20012).

\section{Multi-modality phantom for accuracy verification of image-based navigation tools}

D. Bertsche ${ }^{1}$, A. Reinelt ${ }^{1}$, T. Speidel ${ }^{1}$, P. Metze $^{1}$, K. Stumpf ${ }^{1}$, V. Rasche ${ }^{1}$, I. Vernikouskaya ${ }^{1}$

${ }^{1}$ Ulm University Medical Center, Clinic of Internal Medicine II, Ulm, Germany

Keywords 3D print, silicon phantom, MRI phantom, XR-guidance accuracy validation 


\section{Purpose}

The accuracy of image-based navigation tools is essential for their benefit during an intervention. Validation of such systems requires phantoms with similar properties of the human target structure including the visibility under certain imaging devices. An already introduced patient-specific cardiac phantom focuses on clinical training [1], though not on validation of image-guided techniques. We introduce here the method of creating a patient-specific phantom to validate XR-based navigation tools, allowing identification of unique target points. The phantom was created by $3 \mathrm{D}$-printing of a left atrium (LA) cast model, segmented from a patient-specific CT volume, which was filled with a silicon compound. Predetermined anatomical landmarks could be validated under MRI and XR.

\section{Methods}

Phantom Creation

Patient-specific 3D model of the LA was segmented from CT-dataset (Fig. 1a). The segmented model was modified to obtain an offset between two LA hulls of $4 \mathrm{~mm}$ with the outer layer corresponding to the original segmentation in scale 1:1. A flange was placed at the location of the mitral valve to hold the hulls in place. Besides, 3-mmdiameter connectors were placed annularly around the pulmonary vein pairs to provide "negative" anatomical landmarks. The resulting cast model (Fig. 1b) was printed and subsequently filled with DragonSkinTM 10 MEDIUM mixed with Silicon ThinnerTM (SmoothOn, Macungie, Pennsylvania, USA) in a ratio of $3: 2$ (Fig. 1c). After removing the printout from the dried silicon, separately printed cylinders of height $6 \mathrm{~mm}$ and diameter $3.5 \mathrm{~mm}$ were inserted into negative anatomical landmarks of the silicon model (Fig 1d).

Validation

3D MRI volume of the silicon model was generated at a resolution of $0.34 \mathrm{~mm} / 0.34 \mathrm{~mm} / 1.0 \mathrm{~mm}$ with a T2-weighted TSE sequence on a 3.0T Philips Achieva, R5.6 (Philips Medical Systems B.V, Best, The Netherlands). 12 of the predetermined landmarks were approached under XR (AlluraXper FD20/10, Philips Medical Systems B.V, Eindhoven, The Netherlands) with various $\mathrm{C}$-arm angulations using a TherapieTM Cool PathTM Duo ablation catheter (St. Jude Medical, St. Paul, Minnesota, USA). During landmark approach, the phantom was fixed with tape on the interventional table. To evaluate the feasibility of the phantom for accuracy validation of XR-navigation tools, the 3D landmark positions approached under XR were compared to the target landmark positions determined in the cast model

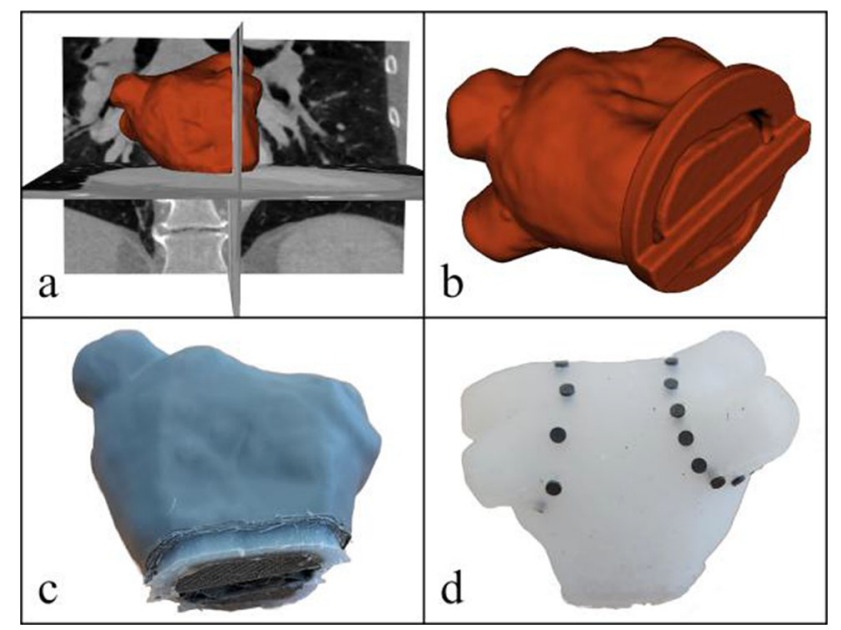

Fig. 1 Phantom creation: segmentation of patient CT (a), cast model (b), printed cast model with removed flange and dried silicon mixture (c), silicon phantom with markers (d) using the average Euclidean distance (aed) and the standard deviation $( \pm)$. Therefore, 3D reconstruction of the catheter tip approaching the landmarks from two differently angulated 2D XR fluoroscopies was done using the 3D-XGuide [2] after manual registration of the 3D MR-Phantom segmentation to XR fluoroscopies. To determine whether deviations already occurred during phantom creation, segmentation and landmark positions in the MRI were additionally compared to the target structures.

\section{Results}

The silicon phantom achieved a measurable MRI signal (Fig. 1a), resulting in an accurate segmentation including the marker positions as non-signal. The thinner amplifies the MR signal and also ensures a lower viscosity and thus a better distribution of the silicon in the casting model. Point cloud comparison of MRI-segmentation and target-segmentation resulted in aed of $0.6 \mathrm{~mm}( \pm 0.4 \mathrm{~mm})$ and a maximum deviation of $2.6 \mathrm{~mm}$ (Fig. 2b). The silicon phantom is visible in XR. In contrast to the inserted printed markers (Fig. 2c), negative markers appear in visible contrast to the silicon model in XR (Fig. 2d), depending on C-arm angulation. Further, evaluation of the accuracy of the phantom based on comparison of the 3D marker positions, resulted in an aed of $1.5 \mathrm{~mm}( \pm 0.6 \mathrm{~mm})$ between XRreconstruction and target-segmentation, $0.9 \mathrm{~mm}( \pm 0.2 \mathrm{~mm})$ between MR-landmarks and target landmarks, and $1.7 \mathrm{~mm}( \pm 0.7 \mathrm{~mm})$ between MR segmentation and XR reconstruction (Fig. 2e). The cutout in the mitral valve provides a good opportunity for marker placement and visibility inside the phantom without distorting the anatomy (Fig. 2f).

\section{Conclusion}

The silicon phantom is clearly visible under MRI and XR, which allows accurate anatomic mimicking of the patient's specific atrium including the identification of anatomical landmarks. The elasticity of the silicon phantom represents a rough approximation of tissue
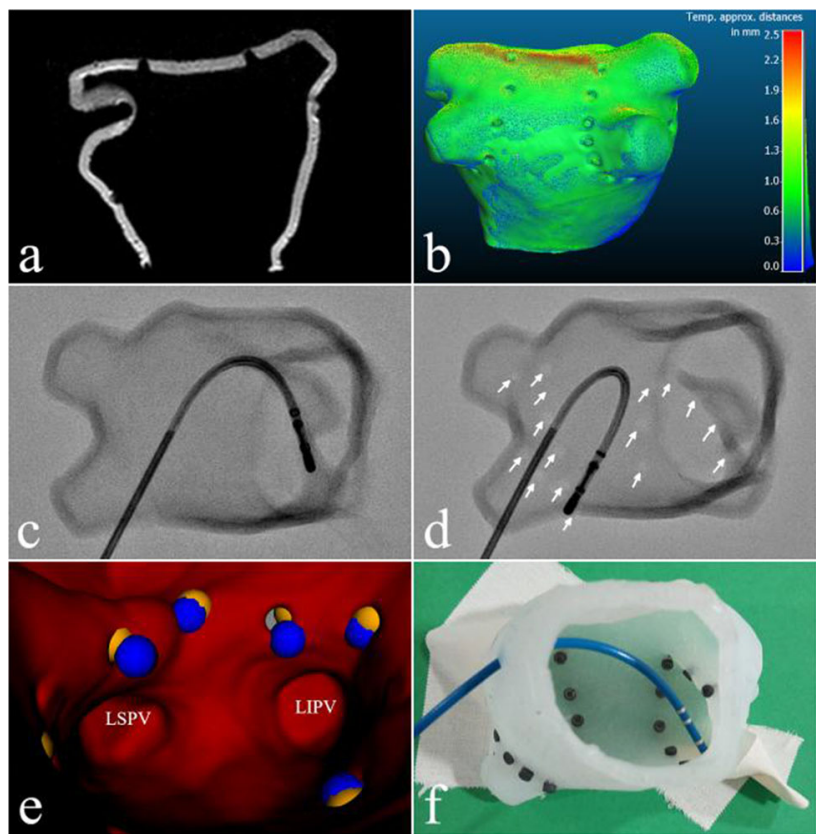

Fig. 2 Results: slice of MR-volume (a), MR-cast model comparison (b), XR of catheter and silicon phantom including printed markers (c) and negative markers (white arrows) (d), comparison of marker positions reconstructed from XR (blue), MR marker positions (yellow), and cast model (red) annularly arranged around left superior and inferior vein (LSPV, LIPV) (e), view during landmark approach (f) 
elasticity and is a property of the silicon compound that allows visibility under MRI. However, the maximal deviation of the MRI-cast model comparison could be attributed to the sinking of the silicon model as a result of the elasticity. Similarly, the deviations in the XR measurements might be related to the elasticity, but also to the manual registration of the phantom model in XR configuration. To reduce the elasticity, a lower proportion of thinner in the silicon compound could be tested. The silicon phantom allowed identification of unique target points with XR and MRI, thus enabling accuracy validation of static XR-based navigation. Additionally, the patient-specific phantom has high accuracy, might enable usage for pre-procedural planning and clinical training.

\section{wledgements}

The project on which this report is based was funded by the Federal Ministry of Education and Research under the funding code 13GW0372C. Responsibility for the content of this publication lies with the author.

\section{References}

[1] Laing J, Moore JT, Vassallo R, Bainbridge D, Drangova M, Peters TM (2018) Patient-specific cardiac phantom for clinical training and preprocedure surgical planning. Journal of Medical Imaging, 5(2):021222

[2] Vernikouskaya I, Bertsche D, Rottbauer W, Rasche V (2020) 3d-xguide: open-source $\mathrm{X}$-ray navigation guidance system. Int $\mathrm{J}$ CARS 16, 53-63 (2021)

\section{Detection of bone metastases on FDG-PET/CT images by using two-step anomaly detection}

H. Yamaguchi ${ }^{1}$, M. Nemoto ${ }^{2}$, H. Kaida ${ }^{3,4}$, Y. Kimura ${ }^{1}$, T. Nagaoka ${ }^{1}$,

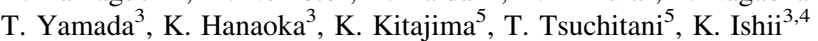
${ }^{1}$ Kindai University, Graduate school of bio-oriented science and technology, Kinokawa, Wakayama, Japan ${ }^{2}$ Kindai University, Faculty of bio-oriented science and technology, Kinokawa, Wakayama, Japan ${ }^{3}$ Kindai University, Institute of Advanced Clinical Medicine, Osakasayama, Osaka, Japan ${ }^{4}$ Kindai University Faculty of Medicine, Department of Radiology, Osakasayama, Osaka, Japan ${ }^{5}$ Hyogo College of Medicine, Department of Radiological

Technology, Nishinomiya, Hyogo, Japan

Keywords bone metastases, FDG-PET/CT, anomaly detection, oneclass SVM

\section{Purpose}

We have been tackling to develop computer-aided detection of cancer metastases on FDG-PET/CT data based on AI anomaly detection [1]. FDG-PET is an effective modality to find metastases of cancer, but such accumulations of FDG to hypermetabolic regions suffer radiologists to read the image. Our previous studies showed that the simple voxel anomaly detections with simple features, such as raw voxel intensities, caused many false positive (FP) voxels due to physiological FDG accumulations. This study proposes a two-step anomaly detection process with Mahalanobis distance-based anomaly detection and anomaly detection using a one-class support vector machine (OCSVM). The proposed method uses not only the raw voxel intensities but also intensity curvature features [2]. We experimented with using the clinical images to investigate the effectiveness of the proposed method.

Methods

Figure 1 shows the flowchart of the proposed method. The proposed method includes a two-step anomaly voxel classification. It consists of (1) a coarse anomaly detection by a Mahalanobis distance to

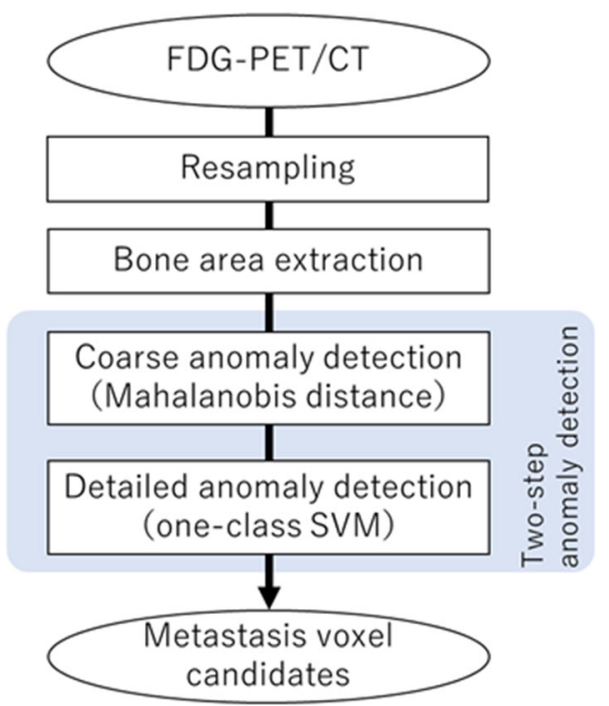

Fig. 1 The flowchart of the proposed method

Table 1 Comparison of detection performances

\begin{tabular}{lll}
\hline & Sensitivity (\%) & FP voxels/case \\
\hline Proposed method & 100 & 131.7 \\
Only Mahalanobis distance & 100 & 473 \\
Only OCSVM & 100 & 1100.9 \\
\hline
\end{tabular}

extract suspicious areas (SAs) and (2) a detailed anomaly detection by using OCSVM to detect lesion voxel candidates from the SAs.

First, CT and FDG-PET images are resampled to $2.4 \mathrm{~mm}$ isotropic resolution. Second, bone areas are extracted from the iso-scaled CT data. The bone area extraction consists of thresholding the HU value, a connected component analysis, and morphological processes. Third, the Mahalanobis distance from normal bone voxel data is measured at each voxel in the extracted bone area. The two features for measuring the Manalanobis distance are voxel intensities of CT image (HU value) and FDG-PET image (SUV). Thresholding the Mahalanobis distance provides the SAs, which are bone voxel clusters with abnormal intensities. Fourth, the detailed voxel analysis using the OCSVM with seven voxel features and an RBF kernel is performed to the voxels in the SAs to detect the metastasis voxel candidates. The seven voxel features are HU value, SUV, two curvature parameters (mean curvature and gaussian curvature) of the HU value surface and the SUV surface, and the Mahalanobis distance measured at the last step. The metastasis voxel candidates are detected by the thresholding for the degree of voxel anomaly calculated using the OCSVM.

Normal distribution parameters for measuring the Mahalanobis distance and the OCSVM are learned in an unsupervised fashion with 29 normal FDG-PET/CT data cases. The hyperparameters of the OCSVM and the thresholds of the two anomaly detections are adjusted experimentally.

In the experiments for evaluating the proposed method, ten clinical FDG-PET/CT data cases, including 19 bone metastases, were used. These data were scanned at Kindai University Hospital and Hyogo College of Medicine Hospital.

\section{Results}

The experimental result shows that the proposed method brought $100 \%$ bone metastasis sensitivity with 131.7 voxels/case FPs. As shown in Table 1, the number of the FPs was smaller than when one of the components of proposed two-step anomaly detection, which was by the Mahalanobis distance or by using the OCSVM, was used. 


\section{Conclusion}

We proposed the detection method of bone metastases on FDG-PET/ CT data using two-step anomaly detection with the Mahalanobis distance calculation and the OCSVM. The evaluation experiments show that the proposed method has an adequate detection accuracy of bone metastasis. Future works include developing the lesion area estimation process based on the detected lesion voxel candidates and developing an accurate lesion area identification using features quantifying the local image pattern characteristics. This work was supported by JSPS KAKENHI Grant Number 20K11944.

\section{References}

[1] Tanaka A, Nemoto M, Kaida H, Kimura Y, Nagaoka T, Yamada T, Hanaoka K, Kitajima K, Tsuchitani T, Ishii K (2020) Automatic detection of cervical and thoracic lesions on FDG-PET/ CT by organ specific one-class SVMs. Int J Computer Assisted Radiology and Surgery 15(Suppl 1): S208-S209

[2] Monga A, S Benayoun (1995) Using Partial Derivatives of 3D Images to Extract Typical Surface Features. Computer Vision and Image Understanding 61(2): 171-189

\section{Benign and malignant non-cystic breast lesion differentiation on the ultrasound image}

\author{
D. Pasynkov ${ }^{1}$, I. Egoshin ${ }^{2}$, A. Kolchev ${ }^{3}$, I. Kliouchkin ${ }^{4}$,
} O. Pasynkova ${ }^{5}$, A. Shefer ${ }^{3}$

${ }^{1}$ Mari State University, Radiology and Oncology, Yoshkar-Ola, Russian Federation ${ }^{2}$ Mari State University, Applied mathematics and informatics, Yoshkar-Ola, Russian Federation ${ }^{3}$ Kazan Federal University, Yoshkar-Ola, Russian Federation ${ }^{4}$ Kazan State Medical University, General Surgery, Kazan, Russian Federation ${ }^{5}$ Mari State University, Fundamental Medicine, Yoshkar-Ola, Russian Federation

Keywords breast lesion, benign, malignant, ultrasound

\section{Purpose}

Breast cancer $(\mathrm{BC})$ remains an important problem for the worldwide healthcare system. Each year more than one million BC cases is diagnosed, and $\mathrm{BC}$ itself represents almost a quarter of all malignancies in women. The highest morbidity values are typical for developed countries and they correspond to more than 360,000 new cases per year in the Europe and more than 200,000 new cases per year in the USA. Moreover in women BC is the most frequently seen malignancy (24.2\% of all malignancies) that responsible for the highest proportion of the cancer-related deaths (15.0\%).

The most relevant strategy to decrease BC-related mortality nowadays corresponds to the wide introduction of mammographic screening. However, along with the improving of ultrasound (US) equipment, they performed large studies, according to which the incremental cancer detection rate for the combination of mammography and US is $2.2-14.2$ (median: 5.2) per 1000 . The majority of the BCs detected by US were less than $1 \mathrm{~cm}$, noninvasive and had no nodal metastases. Such approach can be easily introduced into the population screening programs for women with low or intermediate $\mathrm{BC}$ risk and dense breast parenchyma. However the limitations include the relatively high rate of false positive results that require future assessment and biopsy (BIRADS 3-5 lesions were found in approximately $25 \%$ of women).

During the US examination in case any lesion is found, the first question is to decide if this lesion cystic or solid. On the second step it is important to characterize the solid lesions as benign or malignant, that was the aim of our study.
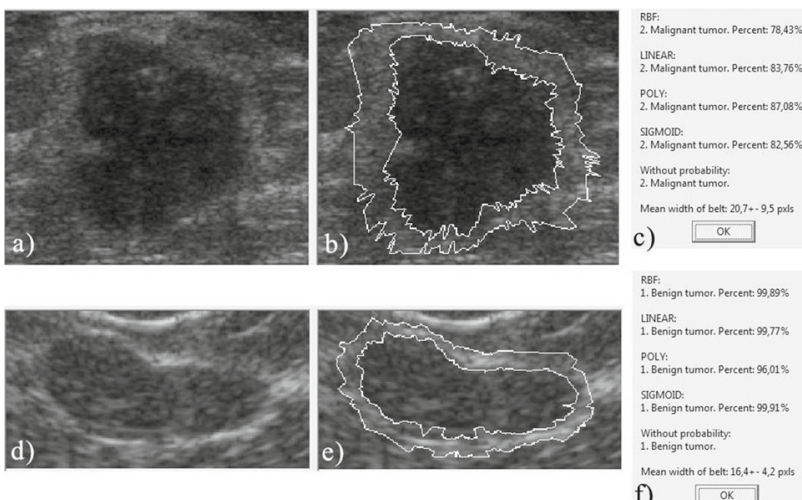

f)

Fig. 1 a, d-Input US images of the malignant and benign breast lesions, respectively; b, e-Segmented lesions and their surrounding belts; $\mathbf{c}, \mathbf{f}-$ Software output for $\mathbf{a}, \mathbf{d}$ images, respectively

\section{Methods}

We used the digital 8-bit ultrasound images of 107 histologically proven (53 malignant and 54 benign, without regard to their histological subtype) breast lesions obtained with the help of the following systems: Siemens-Acuson X150, Esaote MyLab C, Mindray DC-8EX (see Fig. 1a, d).

To characterize the detected lesions as malignant or benign it is proposed to analyze the areas of the image that surround the lesion.

At the first step, the segmenting of the outer contour of the lesion is performed (see Fig. 1b, e) both in semi-automatic and manual (or correction of an automatically selected contour) modes. The features of the gradient difference in the brightness of the image pixels were taken into account to implement the semi-automatic selection of the lesion external area. From the center of lesion, rays were conducted with a given degree of inclination relative to the horizontal axis along the entire circumference. The brightness of the pixels located on the ray was used to calculate the difference of the gradients using one-dimensional filter window of a given size. And the smallest and largest extrema corresponded to the approximate boundaries of the required segmented area.

The border of the selected area was subjected to subsequent correction by filtering the points of the border [1]. If the Euclidean distance between the points of the area border and the nearest points of their regression line is greater than the threshold, then this point was replaced with an interpolated one. Here the threshold was calculated using the Niblack method. This approach allowed to get rid of abrupt changes in the boundaries of the object, which give a false result when selecting the area.

At the second step, the selected area was assigned to one of the lesion groups on the basis of its statistical characteristics of the brightness distribution, textural features (Haralick, Tamura, etc.) [2], as well as geometric features (broadening) of the selected surrounding area of the object. When obtaining textural features, not only the original images were used, but also their transformations with the help of various operators, and the difference between the textural features obtained with different parameters of the algorithms was taken into account.

The support vector machine with different kernels of the trained model was used as a classifier (see Fig. 1c, f).

Finally we compared the rate of differentiation mistakes made by trained radiologist and our software before the biopsy.

\section{Results}

Our approach was able to correctly characterize 49 of $54(90.7 \%)$ benign and 51 of $53(96.2 \%)$ malignant lesions. On the contrary, with the bare eye it was possible to identify correctly 46 of $54(85.2 \%)$ 
benign and 49 of $53(92.5 \%)$ malignant lesions. The corresponding overall specificity values were $90.7 \%$ and $86.0 \%$, respectively.

\section{Conclusion}

Automated approach may surpass the visual assessment performed by trained radiologist that can be clinically relevant.

\section{References}

[1] Williams DJ, Shah M (1992) A fast algorithm for active contours and curvature estimation. CVGIP: Image understanding, 55 (1): 14-26

[2] Humeau-Heurtier A (2019) Texture feature extraction methods: A survey. In IEEE Access, 7: 8975-9000

\section{Improvement of dementia classification accuracy for brain SPECT volumes using the attention mechanism}

N. Matsumoto ${ }^{1}$, A. Saito ${ }^{1}$, M. Fukasawa ${ }^{2}$, T. Iizuka ${ }^{2}$, A. Shimizu ${ }^{1}$

${ }^{1}$ Tokyo University of Agriculture and Technology, Institute of Engineering, Koganei, Tokyo, Japan ${ }^{2}$ Fukujuji Hospital, Center for Dementia, Kiyose, Tokyo, Japan

Keywords SPECT, dementia, deep learning, attention

\section{Purpose}

The number of patients with dementia is increasing rapidly due to the recent super-aging society. Early diagnosis of dementia can help to reduce the incidence of the disease and to determine the optimal treatment for the patient

A single-photon emission computed tomography (SPECT) scan visualizes the distribution of blood flow in the brain. Typical dementia, such as Alzheimer"s disease (AD), dementia with Lewy bodies (DLB), or frontotemporal dementia (FTD), shows reduced blood flow in different parts of the brain. We proposed a convolutional neural network (CNN) [1] for the classification of patients with $\mathrm{AD}$, DLB, and FTD, and healthy controls (HCs).

This paper presents the improvement of the dementia classification accuracy by combining the CNN with an attention mechanism [2], which can lead the attention of the CNN to a specific part of brain.

\section{Methods}

The input SPECT volume was standardized by a three-dimensional stereotactic surface projection in terms of spatial coordinates and gray values, that is, a WSFM image with normalized density values from 0 to 1 . In addition, $\mathrm{Z}$-score volumes were generated by referring to four sites: whole brain, cerebellum, thalamus, and pons. The input of the $\mathrm{CNN}$ is a set of five volumes of a subject, or WSFM and four Z-score volumes.

The proposed network is shown in Figure 1, where the CNN [1] of the perception branch is combined with the attention branch network $(\mathrm{ABN})$ trained using an attention map [2].

First, the whole network was trained by minimizing the following loss function

$L_{a b n}=L_{p e r}+L_{a t t}$

where $L_{p e r}$ and $L_{a t t}$ are cross entropy losses for the perception and attention branches, respectively, computed using classification labels. The trained network generates an attention map $M\left(x_{i}\right)$ of a case $x_{i}$ to emphasize feature maps and visualizes the region of attention.

Next, the ABN was fine-tuned using the loss function below

$L=L_{a b n}+\gamma L_{\text {map }}$

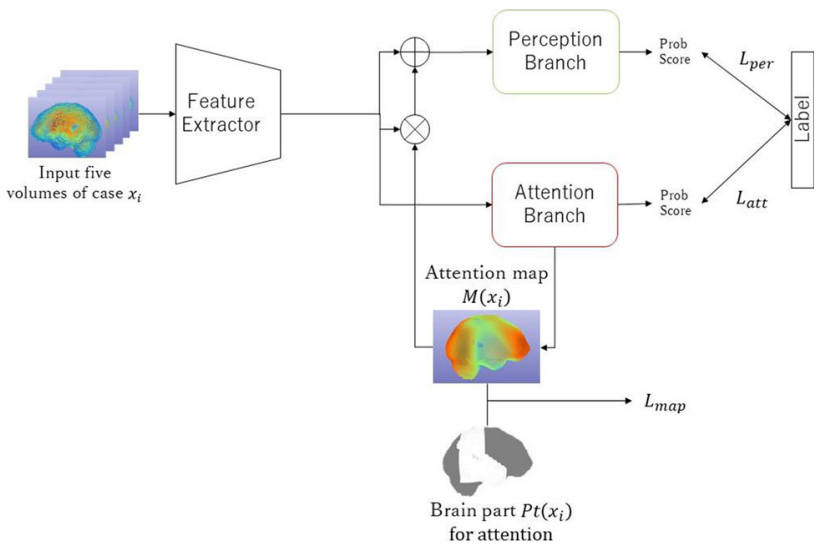

Fig. 1 Proposed network for dementia classification using attention induction

where $\gamma$ is a constant value and $L_{\text {map }}$ evaluates the difference between the attention map and a map manually designed by a user. It is difficult for our study to manually design the teacher map because of the large variety of locations of reduced blood flow that depends on the dementia class. This study proposed a loss $L_{\text {map }}$ that encourages the values of the top $\mathrm{N} \%$ voxels of the attention map to be 1 . Note that $L_{\text {map }}$ is calculated in different brain parts depending on the dementia class, and the brain part $P\left(x_{i}\right)$ was manually specified for each dementia class by the authors in advance. Specifically, the parietal lobe, temporal lobe, and posterior cingulate gyrus are the parts for the AD class; parietal and occipital lobes for the DLB class; frontal and parietal lobes for the FTD class; and none for the HC class.

Results

The materials were 421 SPECT volumes of $60 \times 77 \times 59$ voxels comprising 119 AD, 109 DLB, 93 FTD, and 100 HC cases. We conducted a three-fold cross validation in which the training, validation, and testing volume ratios were $4: 1: 1$. The flip of an input volume was performed as an augmentation with a probability of 0.5 . The Adam optimizer was used in which the initial learning rate for the training of the whole network was 0.001 and it was multiplied by 0.1 at every 100 epochs. In the fine-tuning of $\mathrm{ABN}$ for attention induction, the learning rate was fixed at $0.0001 . \mathrm{N}$ was set as $50 \%$.

Table 1 shows the classification results of the proposed network, and the differences from the conventional network [1] are presented in parentheses. Consequently, the classification accuracy was increased by 2.8 points by the proposed network with attention induction.

\section{Conclusion}

This study presented a method that can induce the attention of the network to the specific brain part. We applied the network trained by the proposed method to the classification of dementia (AD, DLB, and FTD) and HC. The experimental results of the three-fold cross

Table 1 Classification results of the proposed network

\begin{tabular}{lccccll}
\hline True & \multicolumn{6}{l}{ Prediction } \\
\cline { 2 - 7 } & HC & AD & DLB & FTD & $\begin{array}{l}\text { Sensitivity } \\
(\%)\end{array}$ & $\begin{array}{l}\text { Accuracy } \\
(\%)\end{array}$ \\
\hline HC & $79(-6)$ & $8(3)$ & $6(2)$ & $7(1)$ & $79(-6)$ & $80(2.8)$ \\
AD & $6(-3)$ & $94(9)$ & $9(-2)$ & $10(-4)$ & $78(7)$ & \\
DLB & $9(-1)$ & $2(-5)$ & $97(6)$ & $1(0)$ & $88(5)$ & \\
FTD & $10(-4)$ & $11(1)$ & $5(0)$ & $67(3)$ & $72(3)$ & \\
\hline
\end{tabular}


validation demonstrated the effectiveness of the proposed method. Optimization of $\mathrm{N}$ and suppression of attention remain as future works. We will improve the classification accuracy of cases with atypical attention patterns in the future.

\section{References}

[1] Yambe M, Saito A, Fukasawa M, Iizuka T, Shimizu A (2019) 3D deep convolutional neural network using SPECT images for classification of dementia type. CARS 2019-Computer Assisted Radiology and Surgery Proceedings of the 33rd International Congress and Exhibition, 73-74

[2] Mitsuhara M, Fukui H, Sakashita Y, Ogata T, Hirakawa T, Yamashita T, Fujiyoshi H (2019) Embedding Human Knowledge into Deep Neural Network via Attention Map. arXiv preprint arXiv: 1905.03540

\section{Investigation of a high-resolution method for detecting large-intestine regions from plain abdominal CT images}

K. Abe ${ }^{1}$, H. Takeo ${ }^{1}$, S. Nawano ${ }^{2}$, Y. Nagai ${ }^{3}$

${ }^{1}$ Kanagawa Institute of Technology, Atsugi, Kanagawa, Japan

${ }^{2}$ International University of Health and Welfare, Mita Hospital,

Minato-ku, Tokyo, Japan ${ }^{3}$ National Cancer Center Hospital East,

Kashiwa, Chiba, Japan

Keywords CAD, Large-intestine, Segmentation, Plain abdominal CT image

\section{Purpose}

At present, the research \& development of computer-aided diagnosis (CAD) is being conducted in various medical fields. Because of the difficulty in separating the large intestine from peripheral organs, little development has been carried out in the area of CAD that employs CT. Recently, due to improved endoscopic precision and the widespread use of CT colonography, research has been underway into such areas as applying CAD to discover colorectal cancer. However, endoscopy and CT colonography require advance preparation. CT colonography in particular is difficult to conduct in some cases because of stress placed on the colon when the colon is inflated by injecting carbon dioxide gas.

In this study, research \& development was conducted into a method for detecting, at high resolution, the large-intestine region from plain abdominal CT images captured during an abdominal examination. This method makes it possible to acquire images for use in CAD development without necessitating colon-stressing CT colonography. The concordance rate of $71 \%$ between the detection results of this method and manual detection demonstrate this method's detection performance.

Methods

1. Procedural Flow for the Extraction of the Large-intestine Region

Figure 1 shows the procedural flow of the proposed incremental learning method

\section{Preprocessing}

As preprocessing, first-gradation conversion processing was performed to enhance the large intestine. In this study, gradation conversion was performed with the window level set to $50 \mathrm{HU}$ - the ordinary value for the abdomen - and, in consideration of subsequent gas detection, the window width was set to $400 \mathrm{HU}$.

Next, bone-region detection and the detection of gas regions inside the intestine were conducted.

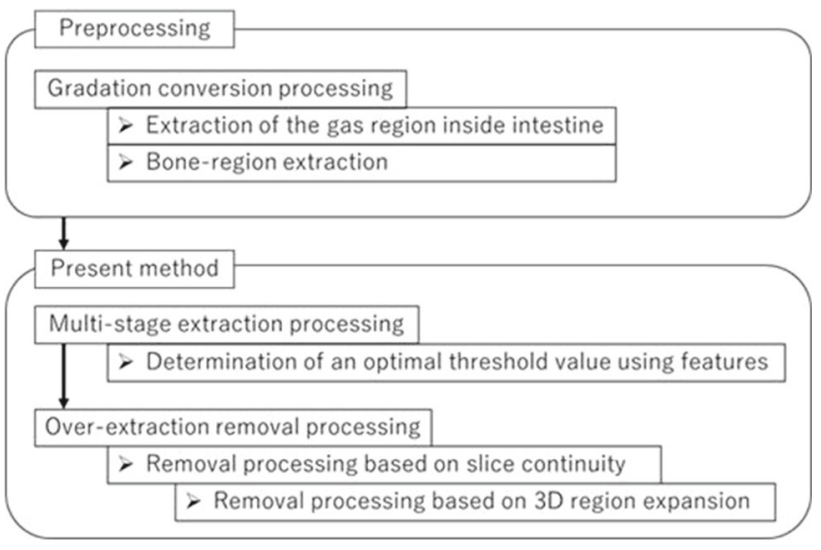

Fig. 1 Large-intestine region extraction procedural flow

During subsequent multi-stage extraction using a threshold value, because of a tendency for incorrect extraction around the spine and some rib areas, bone-region extraction is conducted in advance for use as a feature.

3. Method of Extracting the Large-intestine Region Using Multistage Region Extraction[1, 2].

The multi-step extraction method used for large-intestine extraction is a method for detecting the optimal shape based on the set features by binarization while gradually changing the threshold from a high value to a low value. Digitization is conducted by gradually changing the threshold value from high to low. Detection of the optimal shape is carried out based on the features that have been set. Images for which gradation conversion was performed at widths in the range of $-350 \mathrm{HU}$ to $450 \mathrm{HU}$ using the above gradation process were processed by setting the initial threshold value to $90 \mathrm{HU}$ and then by subtracting in $10 \mathrm{HU}$ increments until $0 \mathrm{HU}$ was reached. Individual seed points are set for each extraction region to reduce the shifting of extraction regions caused by threshold fluctuations. For each of these regions, an optimal threshold value is determined using extraction results for gas regions inside the intestine, bone-region information, circularity and shape features, and features such as centroids. Because there are also cases of the large intestine existing in multiple areas on a slice, individual optimal threshold values are set for each extraction area. This enables the precise extraction of the large-intestine region.

Results

The detection results of the colonic region by this method are shown in Fig. 1 (Left) and the manual extraction results are shown in Fig. 2 (Right) for comparison. The concordance rates of the detection results are $71 \%$, demonstrating high detection performance.

Some of the incorrect detection results can be attributed to looseness in setting the threshold for regions where body surface fat

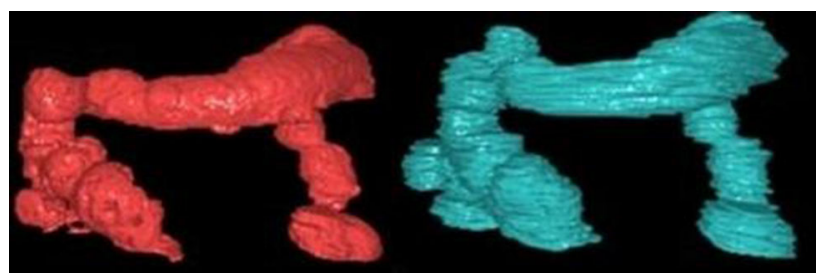

Fig. 2 Detection results of the present. (Left: present method, Right: manual extraction) 
regions, the small intestine, and the large intestine adjoin with other organs.

Conclusion

In this paper, a method for extracting the large-intestine region using plain abdominal CT images was proposed. A multi-stage extraction method enabled the precise extraction of the large intestine, which tends to have faint contrast with peripheral organs. A comparison of the detection results of the present method with manual detection results indicates a concordance rate of $71 \%$, verifying the extraction performance of the present method.

While the present method is capable of extracting the large intestine from plain abdominal CT images, there were no colorectal cancer cases in the samples used in this study. However, the highlevel extraction results obtained with the present method suggest that it can be expected in the future to be applied to colorectal cancer detection methods that use plain abdominal CT images.

\section{References}

[1] Dounomae Y, Takeo H (2013) Image processing method, apparatus and program. Japan patent JP2013-105245A

[2] Abe K, Takeo H, Dounomae Y, Kuroki Y, Nagai Y (2012) Automation of CT-based measurement of 3-dimensional cardiothoracic volumetric ratio, Suppl. 1, CARS2012, pp.S314S315

\section{The relative importance of fine needle aspirate diagnostic attributes in machine learning classification of breast cancer}

S. Raju ${ }^{1}$

${ }^{1}$ UIC College of Medicine at Rockford, Rockford, IL, United States

Keywords breast cancer, cytopathology, machine learning, data mining

\section{Purpose}

The purpose of this study was to use machine learning experiments and information gain ranking to determine the relative importance of several breast mass fine-needle aspirate (FNA) diagnostic features in correctly differentiating malignant from benign breast disease, with the goal of understanding what features may be most important in predicting malignancy.

\section{Methods}

A dataset from the University of Wisconsin consisting of 699 cases was used to train a machine learning algorithm, BayesNet, to correctly classify breast FNA results as benign or malignant on the basis of nine cytopathological attributes: bare nuclei, bland chromatin, cell clump thickness, marginal adhesion, mitoses, normal nucleoli, single epithelial cell size, uniformity of cell shape, and uniformity of cell size [1]. All data analysis was performed using the Weka machine learning platform [2]. Information gain ranking of the attributes was calculated to identify the relative importance of each of the nine cytopathologic attributes in correctly distinguishing malignant from benign lesions. First, the classifier was applied to the dataset with all nine cytopathologic attributes included. Next, the classifier algorithm was applied with attributes successively removed, starting with removal of the attribute with the lowest information gain ranking and continuing through to removal of all but the attribute with the highest information gain ranking. After each successive removal of an attribute from the dataset, the classifier was re-applied, and changes in classifier performance with each attribute reduction were recorded. Three additional experiments were performed: (1) the classifier was applied using only data from lowest-information gain attribute (2) the classifier was applied using only data from the highest-information gain attribute and (3) the classifier was applied using only data from the two highest-information gain attributes. Classifier performance was measured using Area Under the Receiver Operator Curve (AUC), Kappa statistic and percent accuracy of classification. ZeroR, a classifying algorithm that selects the most common classification and applies it to all cases, was used as a control classifier. Stratified tenfold cross validation allowed for the use of one dataset for both classifier training and testing (Fig. 1).

Results

Information gain ranking revealed degree of mitoses as the least valuable attribute for correctly predicting malignancy, while cell size uniformity and cell shape uniformity were determined to be the most important attributes. In order of most to least information gain, the attributes were: cell size uniformity, cell shape uniformity, bare nuclei, bland chromatin, single epithelial cell size, normal nucleoli, clump thickness, marginal adhesion, and mitoses. With all nine attributes included, the AUC was 0.992, with percent correct classification of $97.1 \%$ and Kappa of 0.937 . Removing mitoses from the dataset improved the performance of the classifier by percent correct classification and Kappa statistic, but AUC showed no significant change: upon removal of this attribute, correct classification of malignancy increased to $97.4 \%$, Kappa increased to 0.944, and AUC decreased slightly to 0.991 . Using mitoses as the only attribute in the dataset, the AUC was 0.683 , the percent correct classification was $78.9 \%$, and Kappa was 0.472 . This performance was only marginally better than the performance of ZeroR, the control algorithm, with AUC of 0.496 , percent correct classification of $65.5 \%$, and Kappa of 0 . The greatest decreases in performance by AUC were seen after removal of the 7th attribute, cell shape uniformity, and after the 8th attribute, cell size uniformity. Removal of cell shape uniformity decreased AUC from 0.987 to 0.971 , decreased percent correct classification from $95.4 \%$ to $94.3 \%$, and decreased Kappa from 0.899 to 0.874 . Further removal of cell size uniformity as an attribute decreased AUC to 0.959 , decreased percent correct classification to $92.4 \%$, and decreased Kappa to 0.874. Removing these same two high-information gain attributes from the data first, but maintaining all seven other lower-information gain attributes in the dataset, yielded an AUC of 0.992, a percent correct classification of 97\%, and a Kappa of 00.934 (Table 1).

\section{Conclusion}

In this data set the degree of mitoses does not appears to significantly improve classifier performance when the remaining 8 attributes are available. Using only one or two of the highest information gain ranked attributes results in relatively robust performance of the classifier although there are small improvements in performance when additional attributes are included, with the exception of mitoses.

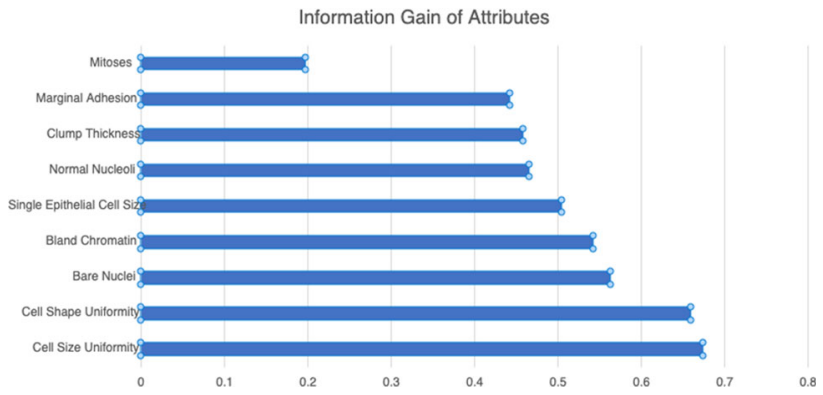

Fig. 1 Comparison of information gain of cytopathologic attributes. Mitoses provided the least useful information, while cell size and cell shape uniformity provided the most useful information for correctly identifying malignancy in breast FNA 
Table 1 BayesNet classifer performance with sequential removal of cytopathologic attributes

\begin{tabular}{|c|c|c|c|c|}
\hline & $\begin{array}{l}\text { Percent } \\
\text { correctly } \\
\text { classified as } \\
\text { malignant or } \\
\text { benign }(\%)\end{array}$ & $\begin{array}{l}\text { Kappa } \\
\text { statistic }\end{array}$ & $\begin{array}{l}\text { Area } \\
\text { under } \\
\text { the curve }\end{array}$ & $\begin{array}{l}\text { Successive } \\
\text { attribute } \\
\text { removed }\end{array}$ \\
\hline All 9 Attributes & 97.10 & 0.937 & 0.992 & None \\
\hline 8 Attributes & 97.40 & 0.9437 & 0.991 & Mitoses \\
\hline 7 Attributes & 97.30 & 0.9406 & 0.991 & $\begin{array}{l}\text { Marginal } \\
\text { Adhesion }\end{array}$ \\
\hline 6 Attributes & 97.30 & 0.9405 & 0.989 & $\begin{array}{l}\text { Clump } \\
\text { Thickness }\end{array}$ \\
\hline 5 Attributes & 96.40 & 0.9214 & 0.988 & $\begin{array}{l}\text { Normal } \\
\quad \text { Nucleoli }\end{array}$ \\
\hline 4 Attributes & 96.30 & 0.918 & 0.989 & $\begin{array}{l}\text { Single } \\
\text { Epithelial } \\
\text { Cell Size }\end{array}$ \\
\hline 3 Attributes & 95.40 & 0.8993 & 0.987 & $\begin{array}{l}\text { Bland } \\
\quad \text { Chromatin }\end{array}$ \\
\hline 2 Attributes & 94.30 & 0.8741 & 0.971 & Bare Nuclei \\
\hline 1 Attribute & 92.40 & 0.8368 & 0.959 & $\begin{array}{l}\text { Cell Shape } \\
\text { Uniformity }\end{array}$ \\
\hline
\end{tabular}

The attributes were removed in order of least information gain to most information gain, and the classifier was re-applied after each removal

Inclusion of mitoses appeared to decrease the performance of the classifier using the kappa statistic and correct classification rate.

\section{References}

[1] Mangasarian OL, Wolberg WH (1990) Cancer diagnosis via linear programming, SIAM News, Vol 23, No 5, Sept 1990, pp 1 $\& 18$

[2] Eibe F, Hall MA, Witten IH (2016) The WEKA Workbench. Online Appendix for Data Mining: Practical Machine Learning Tools and Techniques, Morgan Kaufmann, Fourth Edition

\section{Spine MRI texture analysis and prediction of osteoporotic vertebral fracture}

J. da Silva Ramos ${ }^{1}$, J. Gomes Maciel ${ }^{2}$, A. Juci Traina ${ }^{1}$, P. AzevedoMarques $^{2}$, M. Nogueira-Barbosa ${ }^{2}$

${ }^{1}$ Institute of Mathematics and Computer Science (ICMC), USP, São Carlos, Brazil ${ }^{2}$ Ribeirão Preto Medical School, USP, Medical Imaging, Hematology and Oncology, Ribeirão Preto, Brazil

Keywords Magnetic Resonance Imaging, Textural Attribute, Osteoporosis, Spine

\section{Purpose}

Bone mineral density (BMD) evaluated by bone densitometry (DEXA) is the international reference standard for diagnosing osteoporosis, but DEXA is far from ideal when used to predict secondary fragility fractures [1]. These fractures are strongly related to morbidity and mortality. New techniques are necessary to achieve better prediction of patients at risk to develop fractures. Previous literature showed that spine MRI texture features correlate well with DEXA measurements [2]. Our aim is to evaluate vertebral bone texture analysis searching for potential biomarkers to predict bone fragility fractures secondary to osteoporosis.

\section{Methods}

The study group comprises 63 patients submitted to DEXA and spine MRI: 16 healthy volunteers without osteoporosis and without vertebral fractures, 12 osteopenic patients without fractures and 12 osteopenic with fragility fracture, 12 osteoporotic patients without fractures and 11 osteoporotic with fragility fracture.

T1-weighted (T1w) and T2-weighted (T2w) MRI were segmented for feature extraction (figure 1).

In total, 1316 features were extracted from each vertebral body (L1-L5), including shape and textures features. A few variations were added using $\log$ and wavelets. All features were extracted using pyradiomics.

We performed a binary classification, in which we aimed at predicting if there could be a fracture or not. In total, 97 volumetric vertebral body were previously classified as osteopenia/osteoporosis but had no posterior fracture and 97 volumetric vertebral body were previously classified as osteopenia/osteoporosis and presented a future fracture.

K-nearest neighbor (k-nn), Support Vector Machine (SVM), Trees, Naive Bayes and Discriminant Analysis were tested separately for classification, using tenfolds cross validation. We compared the classifications with and without feature selection. We employed Chi square tests and Principal Component Analysis (PCA) for the selection of features. The hyperparameters of every classifier considered in the experiments were trained so that to achieve the best result. For comparison, we use well-known measures, such as Accuracy, Precision, Sensitivity, F-Measure, and Area Under de Curve (AUC).

\section{Results}

Classification results using the features extracted from T1w and T2w MRI, with binary classification predicting fracture or not are depicted on Table 1. Note that, in general, SVM performed better with selected features, achieving up to $95 \%$ AUC and $89 \%$ Accuracy.

\section{Conclusion}

Texture analysis from spine MRI achieved high diagnostic performance for differentiation of patients with and without vertebral body fragility fracture. The best results were obtained with feature selection and combining texture features extracted both from $\mathrm{T} 1 \mathrm{w}$ and $\mathrm{T} 2 \mathrm{w}$ images. Our results are promising and encourage prospective and longitudinal studies to search for the best MRI features with potential to become biomarkers.

\section{Acknowledgements}

This study was financed in part by the Coordenação de Aperfeiçoamento de Pessoal de Nível Superior (CAPES)_Finance Code 001 and by The São Paulo State Research Foundation (FAPESP) Grants \# 2018/04266-9, 2017/23780-2 and 2016/17078-0.
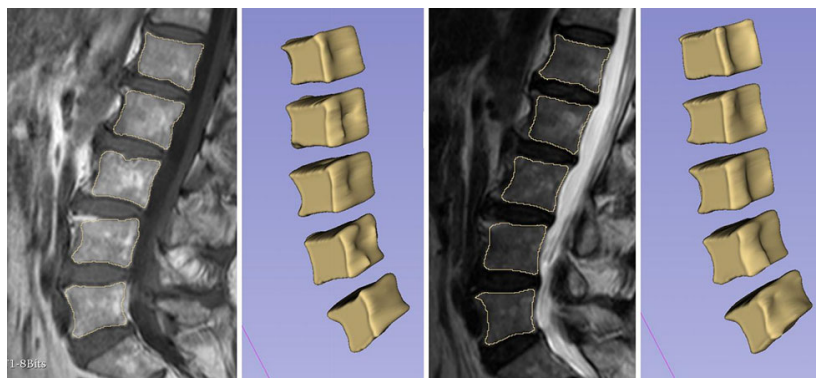

Fig. 1 Example of MRI segmentations for patient 10. a Sagittal T1 weighted MRI. b 3D Segmentation of T1 weighted MRI. c Sagittal T2 weighted MRI. d 3D Segmentation of T2 weighted MRI 
Table 1 Classification results for the extraction of features in $\mathrm{T} 1$ and T2. Binary classification predicting fracture or not

\begin{tabular}{|c|c|c|c|c|c|}
\hline \multirow[t]{2}{*}{ Classifier } & \multicolumn{5}{|c|}{ Measure $(\%)$} \\
\hline & Accuracy & Precision & Sensitivity & $\begin{array}{l}\text { F- } \\
\text { Measure }\end{array}$ & AUC \\
\hline \multicolumn{6}{|c|}{ Without feature selection } \\
\hline k-nn & 84 & 82 & 87 & 84 & 84 \\
\hline SVM & 86 & 82 & 91 & 86 & 95 \\
\hline Tree & 81 & 80 & 84 & 82 & $\underline{90}$ \\
\hline Naïve Bayes & 66 & 61 & 93 & 73 & 67 \\
\hline $\begin{array}{c}\text { Discriminant } \\
\text { Analysis }\end{array}$ & 71 & 69 & 75 & 72 & 71 \\
\hline \multicolumn{6}{|c|}{ With feature selection } \\
\hline k-nn & $\underline{88}$ & 89 & $\underline{88}$ & $\underline{88}$ & 88 \\
\hline SVM & 89 & $\underline{88}$ & 91 & 89 & 95 \\
\hline Tree & 77 & 75 & 80 & 78 & 82 \\
\hline Naïve Bayes & 73 & 71 & 77 & 74 & 82 \\
\hline $\begin{array}{l}\text { Discriminant } \\
\text { analysis }\end{array}$ & 82 & 84 & 78 & 81 & 87 \\
\hline
\end{tabular}

Bold values represent the highest percentages while underlined values represent the second-best result. For the feature-selected results, in total, 69 out of 1316 features were considered

\section{References}

[1] Bauer JS, Link TM (2009) Advances in osteoporosis imaging. Eur J Radiol;71(3):440-9. https://doi.org/10.1016/j.ejrad. 2008.04.064

[2] Maciel JG, Araújo IM, Trazzi LC, Azevedo-Marques PM, Salmon CEG, Paula FJA, Nogueira-Barbosa MH (2020) Association of bone mineral density with bone texture attributes extracted using routine magnetic resonance imaging. Clinics (Sao Paulo); 75:e1766. Epub 2020 Aug 26. https://doi.org/ 10.6061/clinics/2020/e1766

\section{Computerized prediction method for low-grade gliomas with $1 \mathrm{p} / 19 \mathrm{q}$ codeletion in brain MRI images using 3-dimensional attention branch networks}

\author{
D. Tanaka ${ }^{1}$, A. Hizukuri ${ }^{1}$, R. Nakayama ${ }^{1}$ \\ ${ }^{1}$ Ritsumeikan University, Graduate School of Science \\ and Engineering, Kusatsu Shiga, Japan
}

Keywords 3D-attention branch network, low-grade gliomas (LGG), $1 \mathrm{p} / 19 \mathrm{q}$ codeletion, MRI images

\section{Purpose}

Low-grade gliomas (LGG) with co-deficiency of the short arm of chromosome 1 and the long arm of chromosome $19(1 \mathrm{p} / 19 \mathrm{q}$ codeletion) are longer survival and positive response to chemotherapy. Therefore, it is important to diagnose whether the LGG has $1 \mathrm{p} /$ $19 \mathrm{q}$ codeletion or not in planning effective treatment for individual patient. However, the gene analysis for $1 \mathrm{p} / 19 \mathrm{q}$ codeletion requires a lot of time and expense. In previous studies, computerized prediction methods for $1 \mathrm{p} / 19 \mathrm{q}$ codeletion from brain MRI (Magnetic Resonance Imaging) images have been developed using a convolutional neural network $(\mathrm{CNN})$. Those $\mathrm{CNNs}$ trained the relationship between ROIs
(Region of Interest) including an entire tumor and teacher signals (1p/ 19q codeletion or not). Those ROIs included not only the tumor but also background tissue. Therefore, the CNNs might not be trained focusing on the tumor. The purpose of this study was to develop a computerized prediction method for LGG with $1 \mathrm{p} / 19 \mathrm{q}$ codeletion using a 3-dimensional attention branch network (3D-ABN) with an attention mechanism that can extract features focusing on a tumor in brain MRI images.

\section{Methods}

Our database consisted of brain T2-weighted MRI images obtained in 159 patients from The Cancer Imaging Archive (TCIA). Those images included 102 LGGs with $1 \mathrm{p} / 19 \mathrm{q}$ codeletion and 57 LGGs without it. The image size was $256 \times 256$, whereas the number of slices was from 20 to 60 . T2-weighted MRI images first were interpolated to isotropic voxel sizes by a linear interpolation method. A ROI including an entire tumor was manually extracted from T2-weighted MRI images.

Figure 1 shows a proposed 3D-ABN architecture. The 3D-ABN was constructed from a feature extractor, an attention branch, and a recognition branch. The ROIs with a tumor first was resized to $160 \times 160 \times 48$ voxels and then inputted to the input layer of 3D$\mathrm{ABN}$. The feature extractor had four convolutional blocks and extracted feature maps from the input ROI. Each convolutional block composed of the 3D convolutional layer, the batch normalization layer, the rectified linear unit (ReLU) function, and the global average pooling (GAP) layer. The feature extractor generated feature maps for different resolutions from the second, third, and fourth convolutional blocks. The $3 \mathrm{D}-\mathrm{ABN}$ had three attention branches that generated attention maps for weighting the tumor region. The attention maps first were generated by using the feature maps from each attention branch. The attention maps were then applied to the feature maps in the attention mechanisms, as shown in Fig. 1. A tumor segmentation mask was used for modifying the attention map in attention branch 1 . The recognition branch evaluated the likelihood of LGG with $1 \mathrm{p} / 19 \mathrm{q}$ codeletion using the feature maps from the feature extractor. The recognition branch was constructed from three sub-networks consisting of a GAP layer, two fully connected (FC) layers, two dropout layers, and two ReLU functions. The features generated from the three sub-networks were connected in the final FC layer. Finally, the output layer with the softmax function outputted the likelihood of LGG with $1 \mathrm{p} / 19 \mathrm{q}$ codeletion.

A loss function for training the 3D-ABN was defined by a sum of losses for the attention branches and for the recognition branch. The losses for the attention branches and for the recognition were given by mean squared error and a class-balanced cross-entropy with a weight factor $\beta$, respectively. A three-fold cross-validation method was employed to evaluate the classification performance of the proposed $3 \mathrm{D}-\mathrm{ABN}$. In training the proposed $3 \mathrm{D}-\mathrm{ABN}$, the learning rate, the
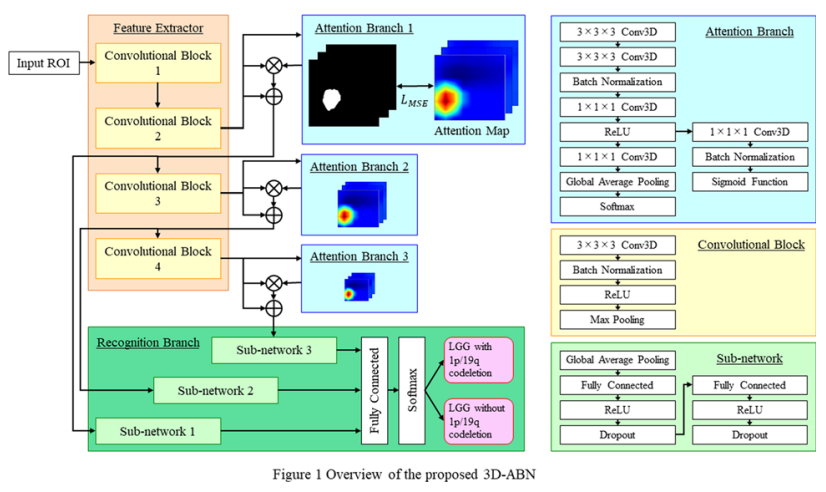

Fig. 1 Overview of the proposed 3D-ABN 
mini-batch size, the number of epochs, the weight factor $\beta$, and the weight decay were set to $0.0001,3,50,0.99$, and 0.01 , respectively. The classification performance of the proposed 3D-ABN was compared with that of a 3D-CNN constructed from four convolutional layers, four batch-normalization layers, four max-pooling layers, and three fully connected layers. The learning parameters for the 3D-CNN were the same as those for the 3D-ABN. The classification accuracy, the sensitivity, the specificity, the positive predictive value (PPV), the negative predictive value (NPV), and the area under the receiver operating characteristic curve (AUC) were used as the evaluation indices of the classification performance.

\section{Results}

The classification accuracy, the sensitivity, the specificity, the PPV, the NPV, and the AUC with the proposed 3D-ABN were $78.0 \%$ (124/ 159), $79.4 \%$ (81/102), 75.4\% (43/57), 85.3\% (81/95), 67.2\% (43/64), and 0.804 , respectively. All evaluation indices for the proposed 3D$\mathrm{ABN}$ were greater than those for the 3D-CNN $(68.6 \%, 75.5 \%, 56.1 \%$, $75.5 \%, 56.1 \%$, and 0.736 ). The AUC for the $3 \mathrm{D}-\mathrm{CNN}$ was improved significantly by the proposed 3D-ABN $(p<0.014)$. These results implied that the proposed 3D-ABN was effective in predicting LGG with $1 \mathrm{p} 19 \mathrm{q}$ codeletion when compared as the 3D-CNN.

\section{Conclusion}

In this study, we developed a computerized prediction method for LGG with $1 \mathrm{p} / 19 \mathrm{q}$ codeletion in brain MRI images using the 3D-ABN. The proposed method was shown to have a high classification performance for $1 \mathrm{p} / 19 \mathrm{q}$ codeletion and would be useful in planning effective treatment for LGG.

\section{Deep learning-based prediction of clinical outcomes in diffuse large B-cell lymphoma (DLBCL) from a baseline 18F-FDG PET scan}

P. $\mathrm{Liu}^{1}$, X. Gao ${ }^{1}$, G. Zheng ${ }^{1}$

${ }^{1}$ Institute of Medical Robotics, School of Biomedical Engineering, Shanghai Jiao Tong University, ShangHai, China

Keywords Diffuse Large B-cell Lymphoma, Prognosis, Radiomics, Deep learning

\section{Purpose}

Early identification of Diffuse Large B-cell Lymphoma (DLBCL) patients with a poor prognosis from baseline 18F-FDG PET/CT scans allows for the tailing of their curative remediation plan for an improved chance of cure. However, this task often challenge since it suffers from the problem of insufficient labeled data and severe class imbalance. Deep learning-based algorithms have recently gained momentum for a number of computer-aided diagnosis and prognosis applications. In this work, we propose a novel multi-task deep learning (DL)-based method for joint segmentation and predictive of 2-year progression-free survival (2y-PFS) of DLBCL, enabling outcome prognostication directly from baseline 18F-FDG PET/CT scans. Furthermore, in order to tackle the problem of data insufficient and class imbalance, we introduce Batch Nuclear-norm Maximization loss to the prediction matrix.

Methods

In this paper, we propose a multi task learning method for PET images. The proposed method do the segmentation and classification task in a end-to-end deep learning model, this deep learning model takes PET volumes as inputs and produces two outputs, including a volume-level prediction probability and a segmentation map. The proposed network is shown in Fig. 1. We adopt U-Net as the backbone network as it achieves excellent performance in 3D medical image analysis. There are three modules in U-Net architecture: (i) an encoding module, (ii) a decoding module, and (iii) skip connections between encoding module and decoding module. As shown in

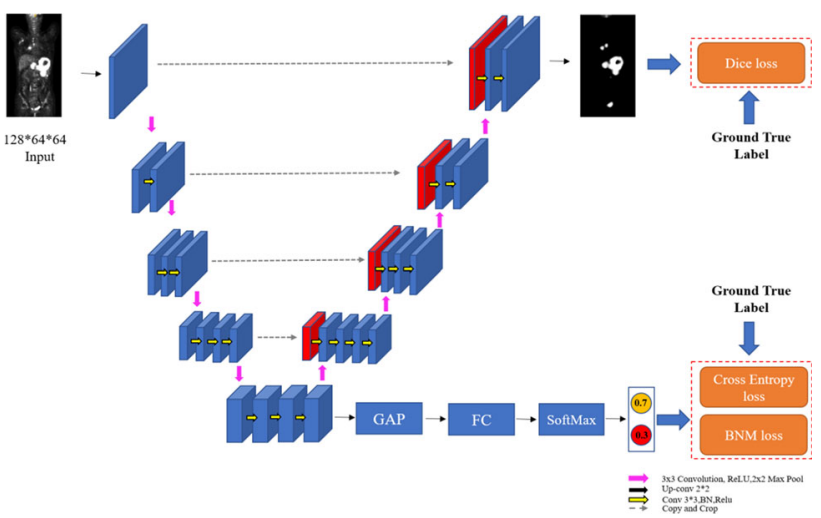

Fig. 1 Overall architecture of our method

Fig. 1q, there are four down-sampling operations in the encoding module to extract high-level semantic features. Symmetrically, there are four up-sampling operations in the decoding module to interpret the extracted features from the encoding module to predict the 3D segmentation map. Skip connections connect feature maps of same level from the encoding module to the decoding module to propagate spatial information and refine segmentation convolution layer. We set all convolution kernel sizes to $3 \times 3 \times 3$, every convolution operation followed with batch normalization $(\mathrm{BN})$ and rectified linear unit (ReLU). We use Max-pooling to down-sample feature maps.

In our Multi-task U-Net architecture, we share the encoding module for classification and segmentation task to extract common features for those two tasks. As illustrated in Fig. 1, a classification branch is added to the bottom of the U-Net, Firstly, feature maps from bottom of the U-Net are fed into the classification network which has a convolution layer and a fully connected (FC) layers, and followed with one softmax layer to predict the input volume as 2-year progression-free survival (2y-PFS) of DLBCL. Since our task is label insufficient and suffer from class imbalance problem. In order to tackle this issue, we reinvestigate the structure of classification output matrix of a randomly selected data batch.

As [1] find by theoretical analysis that the prediction accuracy could be improved by maximizing the nuclear-norm of the batch output matrix. Accordingly, to improve the classification performance, we introduce Batch Nuclear-norm Maximization (BNM) on the output matrix. BNM could boost the performance under label insufficient learning scenarios.

A cohort study containing clinical data including baseline $18 \mathrm{~F}$ FDG PET/CT scans of 124 patients was used for the evaluation of the proposed method under a five-fold cross validation scheme.

Results

We use Accuracy, area under the receiver operating characteristic (ROC) curve (area under the curve [AUC]), sensitivity and specificity for quantitative evaluation of classification performance. For segmentation, we use Dice similarity coefficient (DSC) to evaluate the performance. We compare our method with two method: the alternative radiomic model and the model without BNM. As shown in Table 1, our multi-task learning method achieves consistent improvements on all metrics over radiomics model, this showing that our multi-task deep learning model could learn better representation of the PET images for classification and segmentation. Furthermore, the multi-task learning framework could do the segmentation in the same time. By introduce the BNM for our multi-task deep learning model, we improve the AUC, Specificity and Dice, especially with significant improves on Specificity and Dice, showing that the BNM is indeed helpful for more effective tackle of this label insufficient situation. 
Table 1 Performance of three models when evaluated with fivefold cross validation

\begin{tabular}{llllll}
\hline Methods & AUC & Accuracy & Sensitivity & Specificity & DICE \\
\hline Radiomics model & 0.735 & 0.718 & 0.856 & 0.353 & - \\
Multi-task U-net & 0.792 & $\mathbf{0 . 8 1 4}$ & $\mathbf{0 . 8 7 5}$ & 0.622 & 0.964 \\
Multi-task U-net & $\mathbf{0 . 8 1 8}$ & $\mathbf{0 . 8 1 4}$ & 0.846 & $\mathbf{0 . 7 2 5}$ & $\mathbf{0 . 9 9 2}$ \\
with BNM & & & & & \\
\end{tabular}

\section{Conclusion}

The proposed multi-task deep learning prognostic model achieved much better results than the alternative radiomic model. Our experimental results demonstrated that the proposed model has the potential to be used as a tool for tumor segmentation of PET images and risk stratification for patients with DLBCL.

\section{References}

[1] Cui S, Wang S, Zhuo J, Li L, Huang Q, Tian Q (2020) Towards discriminability and diversity: Batch nuclear-norm maximization under label insufficient situations. In: Proceedings of the IEEE/CVF Conference on Computer Vision and Pattern Recognition. pp. 3941-3950

\section{A Novel 2D Augmented Reality Ultrasound Framework using an RGB-D Camera and a 3D-printed Marker}

\author{
Y. Zhou ${ }^{1}$, G. Lelu ${ }^{1}$, B. Labbé ${ }^{1}$, G. Pasquier ${ }^{1}$, P. Le Gargasson ${ }^{1}$, A. \\ Murienne $^{1}$, L. Launay ${ }^{1}$ \\ ${ }^{1}$ Institut de recherche technologique b-com (IRT b-com), Immersive \\ \& Medical Technologies Lab, Cesson-Sévigné, France
}

Keywords Ultrasound, Augmented reality, Probe calibration, RGB-D camera

\section{Purpose}

2D ultrasound (US) is widely used in many clinical practices such as needle biopsy and surgery guidance since it is real-time, safe and cheap. Images are acquired using a handheld probe, resulting in a series of 2D slices, which must be further related to the underlying 3D anatomy of the patient for effective navigation. This could be accomplished by simultaneously acquiring $2 \mathrm{D}$ images and tracking the probe using an optical or electromagnetic (EM) tracking system. Moreover, an US Probe Calibration (UPC) procedure should be performed beforehand in order to get the spatial relationship between the image plane and the probe.

An important component of the Augmented Reality (AR) US frameworks is the underlying tracking system. Many commercial solutions exist, for instance the NDI Polaris/Aurora. A complex UPC procedure is generally necessary, during which multiple sensors are attached to the calibration phantom, the probe, as well as the stylus which is an auxiliary tool used to localize the phantom. The calibration matrix can be estimated using the open-source software PLUS toolkit. Such classical AR US frameworks usually ensure a high precision level. However, a costly, complex, and somewhat cumbersome system is required, which might hamper their utilization in clinical routine.
Recently, in [1], the authors introduced a simpler and cheaper AR US system based on a standard RGB-D camera. The camera is fixed upon the probe, and its pose is estimated from contextual image information. The cost is much reduced. However, textured scenes are required and multiple fiducial markers should be installed in the Operating Room (OR). This could have a non-negligible impact on the routine clinical environment.

In this paper, we propose a simple and low cost RGB-D camerabased AR US framework. A specifically-designed 3D-printed marker and a fast model-based 3D point cloud registration algorithm FaVoR [2] are seamlessly merged. Unlike in [1], the tracking is merely based on depth information. This is aimed at obtaining a stable solution despite the constraints related to the OR, such as the strong OR light which can sometimes saturate the RGB sensors. In addition, the UPC procedure is much simplified, additional sensors or tools, such as the stylus, are no longer needed.

\section{Methods}

In AR US, a first step is to perform US probe calibration. Figure 1 illustrates our calibration system using the $\mathrm{N}$-wire phantom $f$ Cal-2.1. Nine metal wires of $1 \mathrm{~mm}$ diameter were installed so as to form the "N" shape at three different depth levels. The patent-pending marker actually results from a sophisticated combination of small cubes of size $1 \mathrm{~cm}$, forming an object of roughly $4 \mathrm{~cm}$ span. Both the RGB-D camera and the US image stream are connected to the mobile workstation via USB 3.0 cables, and are managed by the $\mathrm{fCal}$ application of PLUS.

The calibration consists of three key steps: phantom localization, probe tracking and PLUS calibration. Unlike most calibration procedures where additional markers need to be attached to the phantom for its localization, we make full use of the generic nature of FaVoR and directly register the virtual model of the phantom with the 3D point cloud (depth map). Then, FaVoR is reparametrized with the probe marker's virtual model for the tracking. The probe is placed over the phantom to image the installed fiducial wires, producing US images with nine visible white spots. Those points are segmented by PLUS, and the middle-wire points are matched to the groundtruth positions in the phantom. Since both the phantom and the probe marker are localized by the same camera, spatial positions in the phantom space are easily mapped to 3D coordinates in the marker's space. This generates correspondences between the image and marker spaces, from which is further computed the calibration matrix via least-square fitting. Once calibrated, our system is deployed to augment a real-world scenario with US image information. A visualization software based on OpenGL has been developed for rendering an US image within a standard RGB image.

\section{Results}

The distance between the RGB-D camera and the top of the phantom is a key variable that could impact the accuracy. As a result, we performed the calibration at five different distances. The magnitudes of the calibration errors on N-wire phantom generated by PLUS are listed in Table 1.

We observe that the Occipital Structure Core achieved its best accuracy at $50 \mathrm{~cm}$, corresponding to an average calibration error of $2.6 \mathrm{~mm}$. Figure 2 shows the deployment of the calibrated probe for augmenting a real-world video on a BluePrint phantom. A needle insertion procedure was simulated. The US images are rendered using a proper color map which highlights pixels with higher intensities in bright yellow. A spatial continuity is observed between the needle 

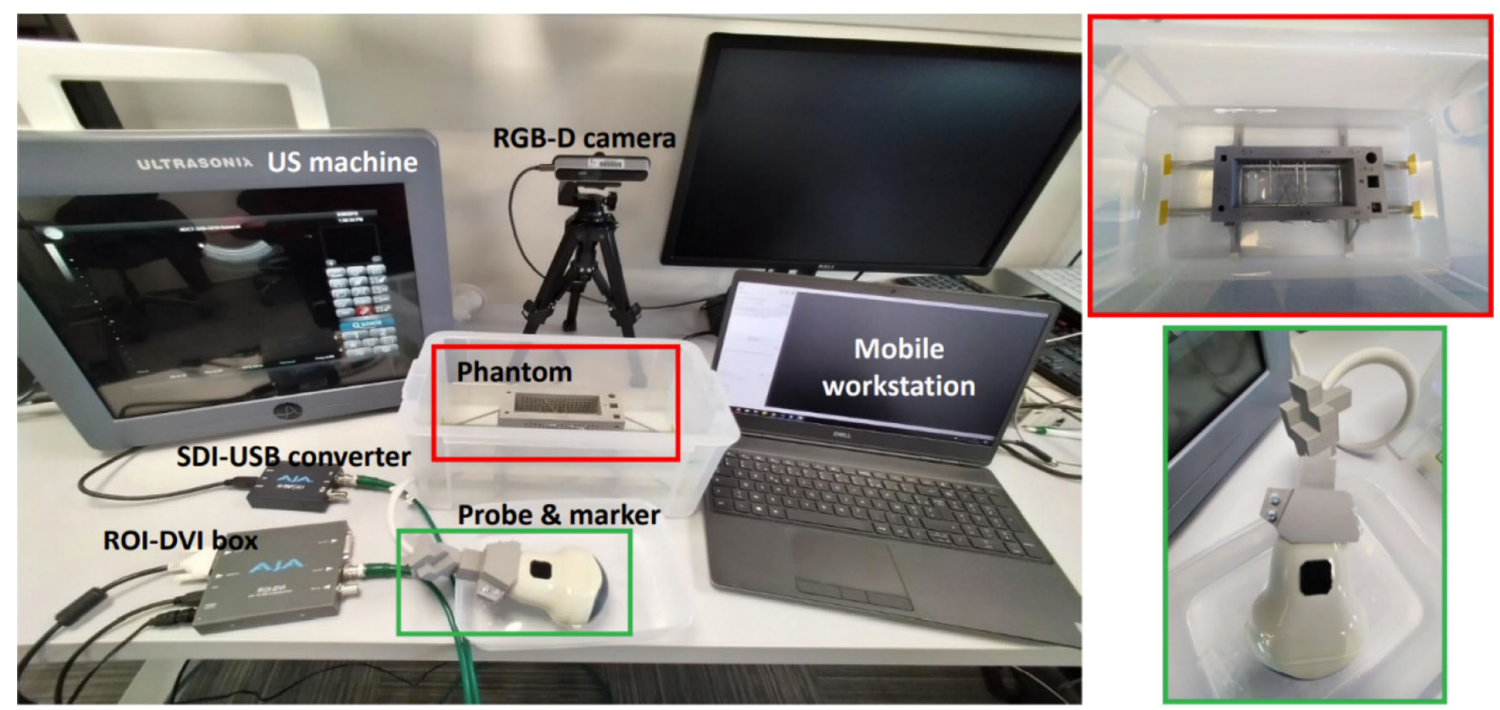

Fig. 1 The ultrasound probe calibration setting of the proposed AR US framework. An Ultrasonix US machine, an Occipital Structure Core RGB-D camera, a 3D-printed marker, a water tank containing the $\mathrm{N}$-wire phantom and a mobile workstation are combined for the calibration

Table 1 Evolution of the calibration error over the camera-phantom distance

\begin{tabular}{llllll}
\hline $\begin{array}{l}\text { Distance } \\
(\mathrm{cm})\end{array}$ & 40 & 50 & 60 & 70 & 80 \\
\hline $\begin{array}{c}\text { Calib. Err. } \\
(\mathrm{mm})\end{array}$ & $30.1 \pm 20.0$ & $2.6 \pm 0.4$ & $3.4 \pm 0.5$ & $3.3 \pm 0.7$ & $4.0 \pm 0.6$ \\
\hline
\end{tabular}

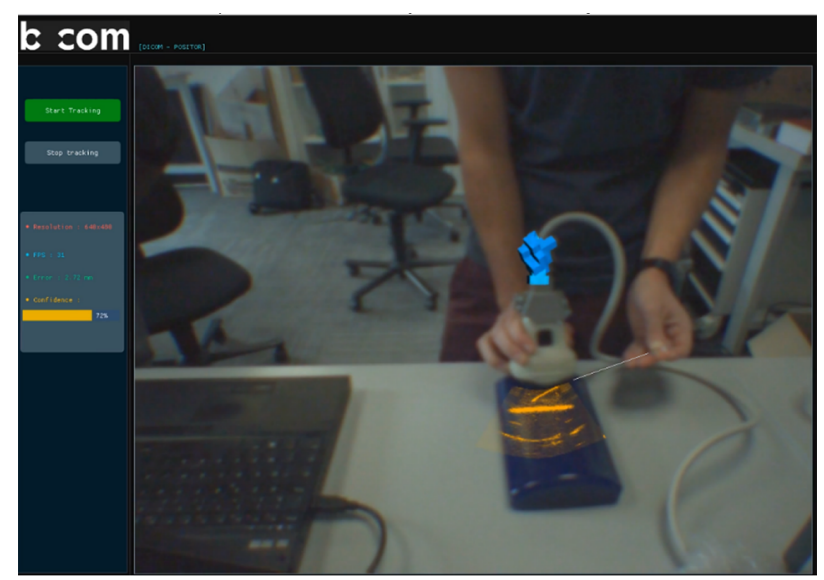

Fig. 2 Augmented reality ultrasound simulating needle insertion in real-world video. The tracked marker is rendered in blue and the calibrated US image is displayed on the RGB image part which is outside the phantom (highlighted for better visualization) and the inside part revealed by US.

\section{Conclusion}

A simple and low cost AR US framework including an RGB-D Camera, a 3D-printed marker, and a fast point-cloud registration algorithm FaVoR was developed and evaluated on an Ultrasonix US system. Preliminary results showed a mean calibration error of $2.6 \mathrm{~mm}$. The calibrated probe was then used to augment a real-world video in a simulated needle insertion scenario. Visually-coherent results were observed. Future work should include a more rigorous and thorough validation of the proposed method, through quantitative evaluations in both simulated and real world medical scenarios.

\section{References}

[1] Busam B, Ruhkamp P, Virga S (2018) Markerless inside-out tracking for interventional applications. http://arxiv.org/ abs/1804.01708

[2] Murienne A, Labbé B, Launay L (2020) Model-based 3D Tracking for Augmented Orthopedic Surgery. EPiC Series in Health Sciences 4:197-200

Publisher's Note Springer Nature remains neutral with regard to jurisdictional claims in published maps and institutional affiliations. 